\title{
Malaria rapid diagnostic tests : Laboratory aspects in the diagnostic setting
}

Citation for published version (APA):

Gillet, P. (2011). Malaria rapid diagnostic tests : Laboratory aspects in the diagnostic setting. [Doctoral Thesis, Maastricht University]. Universiteit Maastricht; CAPHRI. https://doi.org/10.26481/dis.20110622pg

Document status and date:

Published: 01/01/2011

DOI:

10.26481/dis.20110622pg

Document Version:

Publisher's PDF, also known as Version of record

\section{Please check the document version of this publication:}

- A submitted manuscript is the version of the article upon submission and before peer-review. There can be important differences between the submitted version and the official published version of record.

People interested in the research are advised to contact the author for the final version of the publication, or visit the DOI to the publisher's website.

- The final author version and the galley proof are versions of the publication after peer review.

- The final published version features the final layout of the paper including the volume, issue and page numbers.

Link to publication

\footnotetext{
General rights rights.

- You may freely distribute the URL identifying the publication in the public portal. please follow below link for the End User Agreement:

www.umlib.nl/taverne-license

Take down policy

If you believe that this document breaches copyright please contact us at:

repository@maastrichtuniversity.nl

providing details and we will investigate your claim.
}

Copyright and moral rights for the publications made accessible in the public portal are retained by the authors and/or other copyright owners and it is a condition of accessing publications that users recognise and abide by the legal requirements associated with these

- Users may download and print one copy of any publication from the public portal for the purpose of private study or research.

- You may not further distribute the material or use it for any profit-making activity or commercial gain

If the publication is distributed under the terms of Article $25 \mathrm{fa}$ of the Dutch Copyright Act, indicated by the "Taverne" license above, 


\section{Malaria Rapid Diagnostic Tests: Laboratory aspects in the diagnostic setting}

Philippe Gillet

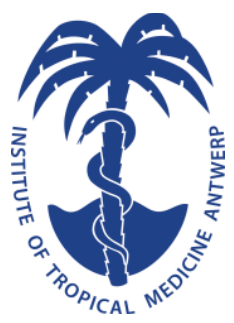

\section{Caphri School for Public Health and Primary Care}

Contact:

Philippe Gillet, ITM, Department of Clinical Sciences, pgillet@itg.be, TEL. +32(0)3 2476347

ISBN: 978-2-930615-02-8

(C) copyright Philippe Gillet, Antwerp, 2011 



\title{
Malaria Rapid Diagnostic Tests: Laboratory aspects in the diagnostic setting
}

\author{
DISSERTATION
}

to obtain the degree of Doctor at the Maastricht University, on the authority of the Rector Magnificus, Prof. dr. G.P.M.F. Mols, in accordance with the decision of the Board of Deans, to be defended in public on Wednesday 22 June 2011, at 16.00 hours

Philippe Gillet 
Supervisor:

Prof. dr. C.A. Bruggeman

Co-supervisor:

Prof. dr. J. Jacobs, Institute of Tropical Medicine Antwerp, Belgium

Assessment Committee:

Prof. dr. M.P. van Dieijen-Visser

dr. G. Schreij

Prof. dr. C.D.A. Stehouwer

Prof. dr. A.J.A.M. van den Ven,

International Health, Radboud Universiteit, Nijmegen 


\section{Contents}

Chapter I: Introduction and outlines of this thesis............................. 3

Chapter II: Laboratory evaluation of malaria RDTs targeting

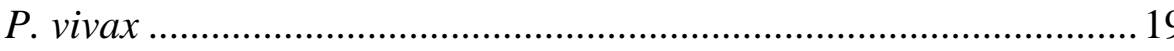

IIa: Laboratory evaluation of malaria RDTs targeting $P$. vivax ................21

IIb: Laboratory evaluation of malaria RDTs targeting $P$. vivax .................35

Chapter III: Assessment of the prozone effect in malaria RDTs....... 49

IIIa: Assessment of the prozone effect in malaria RDTs ............................51

IIIb: Assessment of the prozone effect in malaria RDTs ..........................65

Chapter IV: Buffer substitution in malaria rapid diagnostic tests causes false-positive results...................................................... 87

Chapter V: External quality assessment on the use of malaria rapid diagnostic tests in a non-endemic setting 103

Chapter VI: Malaria rapid diagnostic kits: quality of packaging, design and labelling of boxes and components and readability and accuracy

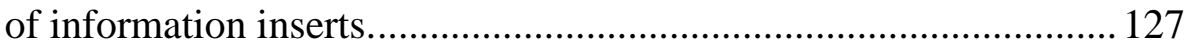

Chapter VII:

Summary, general discussion and future perspectives .................... 157

Samenvatting, algemene discussie en toekomst perspectieven ........ 167

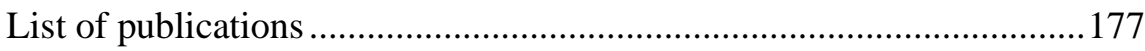

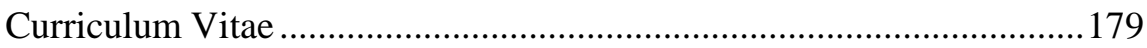

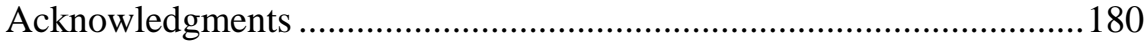





\section{Chapter I}

\section{Introduction and outlines of this thesis}




\section{Malaria Rapid Diagnostic Tests (RDTs):}

Laboratory aspects in the diagnostic setting

\section{Chapter I: Introduction and outlines of this thesis}

\section{Malaria: an overview}

Malaria is caused by a parasite called Plasmodium, which is transmitted through the bites of infected mosquitoes. In the human body, the parasites multiply in the liver, from where they spread to infect red blood cells. Five Plasmodium species have shown to infect humans: Plasmodium falciparum, Plasmodium vivax, Plasmodium ovale, Plasmodium malariae and Plasmodium knowlesi.

According to the malaria report 2010 of the World Health Organization (WHO), malaria is currently endemic in over 100 countries with 3 billion people at risk of infection and around 225 million cases in 2009, leading to approximately 781,000 deaths [1]. P. falciparum was among the leading causes of death worldwide from a single infectious agent. $P$. falciparum is mostly confined to tropical climates; $P$. vivax also occurs in subtropical areas but is rare in Central-Africa, where P. ovale is prevalent. $P$. malariae is rare and $P$. knowlesi, the most recently described "zoonotic" species, is restricted to South-East Asia. Figure 1shows the global distribution of malaria in the world.

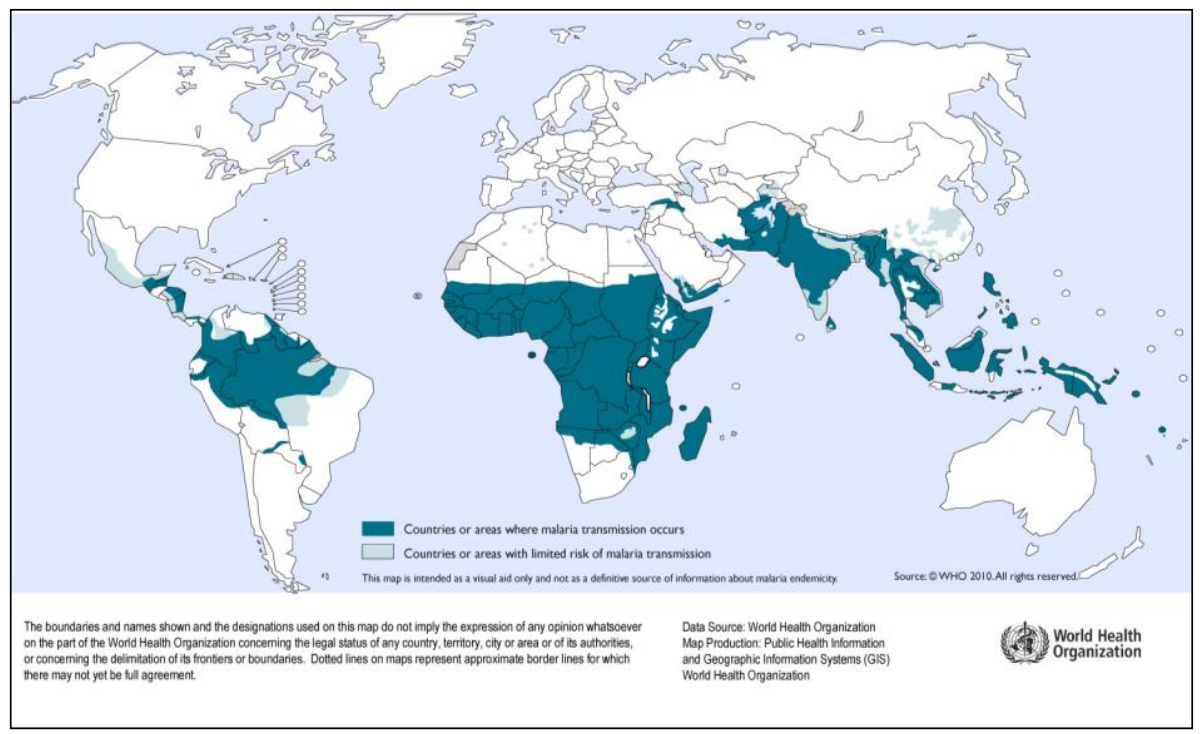

Figure 1: Countries or areas at risk of malaria transmission in 2009 (WHO 2010). 
Plasmodium has a sexual cycle inside the mosquito and an asexual cycle inside men. During the bite of an infected mosquito, Plasmodium sporozoites are injected in the blood and will move to liver cells where they will develop into liver schizonts. After the rupture of the schizonts, thousands of merozoites are released into the bloodstream and each of them will invade a red blood cell (RBC). There they will develop into trophozoites which will mature into schizonts that on their turn will divide into new merozoites which will invade new RBCs. After one or two weeks, gametocytes will be produced. These will continue the sexual cycle when taken up by a mosquito during a next blood meal. As to the asexual cycle, liver schizonts of $P$. vivax and $P$. ovale may persist for months in the liver before releasing merozoites into the blood. The persistent ("dormant") forms are called hypnozoites; the delayed symptoms are called relapses (Figure 2).

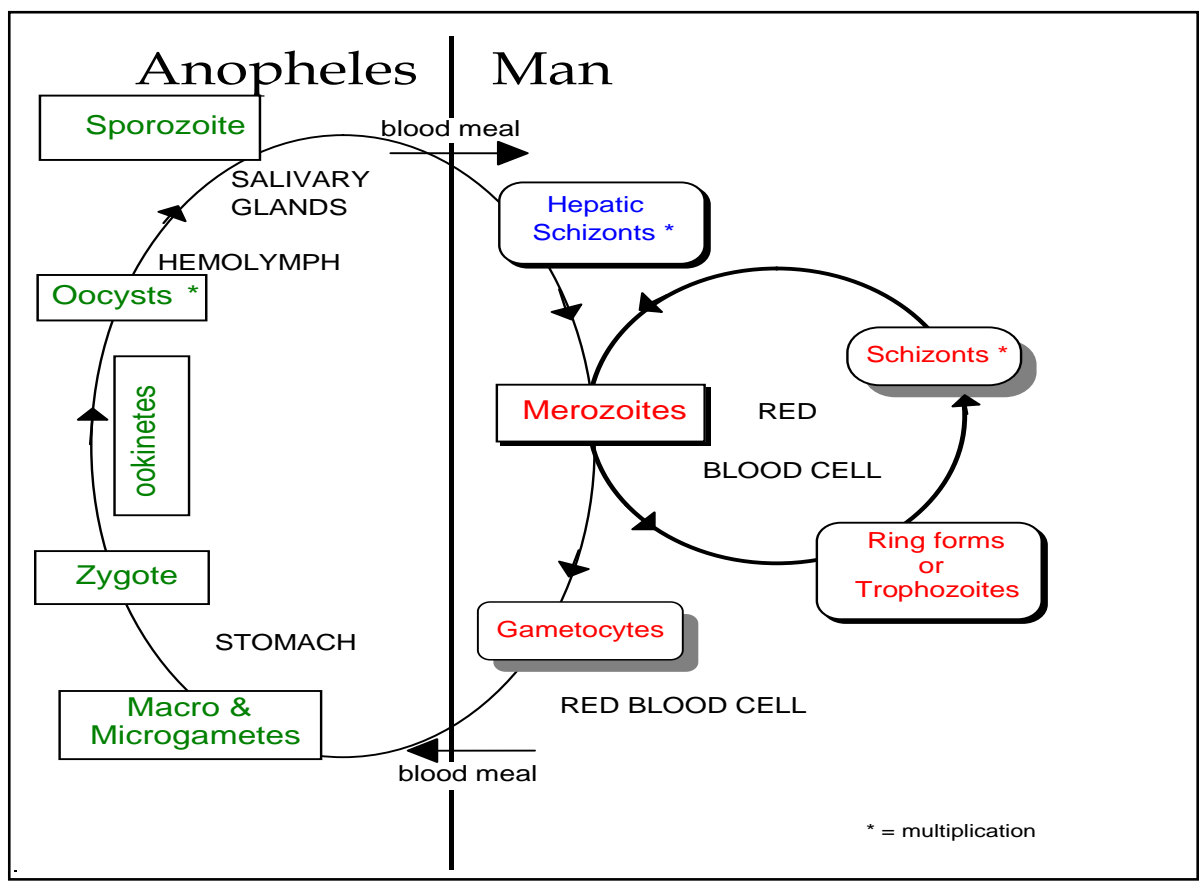

Figure 2: Life cycle of human Plasmodium species (adapted from Wéry) [2].

Small children and pregnant women are particularly vulnerable to malaria. Likewise, also non-exposed travellers are vulnerable and each year, 10,000 malaria cases are reported among returned international travellers, although their real number is estimated at 30,000[3].

Symptoms of malaria may vary and include fever, headache, aches and pains elsewhere in the body and occasionally abdominal pain, diarrhoea and vomiting. If not treated, malaria can quickly become life-threatening. This is especially the case 
for $P$. falciparum which unlike $P$. vivax/P. ovale and $P$. malariae is not restricted to young or old RBCs and can cause elevated parasite densities. In addition, infected RBCs stick to the vascular endothelium, leading to obstructed blood flow in the organ capillaries and lethal complications such as cerebral malaria, pulmonary oedema and impairment of liver and renal functions. Prompt diagnosis is essential for the treatment and outcome of P. falciparum. P. vivax accounts for almost half of the malaria infections worldwide and is no longer considered as a mild infection: complicated infections have been demonstrated in both endemic countries and in returned travellers $[4,5]$ and the species is more difficult to eradicate than $P$. falciparum [6].

\section{What is essential in the laboratory diagnosis of malaria?}

Prompt treatment of malaria requires accurate and prompt diagnosis. Diagnosis based on clinical symptoms is notoriously non-specific and WHO now recommends parasite-based diagnosis for all patients [7]. The cornerstone of malaria diagnosis in the laboratory is microscopy. Plasmodium parasites are diagnosed by microscopic examination of a thick blood film, which consists of a superposition of several layers of blood cells. The red blood cells are lysed during the staining process. Thin blood films represent a monolayer of blood, allowing observation and assessment of the red blood cell's shape and inclusions and clear distinction of the different parasites, allowing species identification. Characteristics of RBC (dimensions, shape, inclusions and numbers infected) and Plasmodium parasites (size, stages, and dimensions) allow distinction between the different Plasmodium parasites. The differential diagnosis between $P$. ovale and $P$. vivax is notoriously difficult [8]. The parasite density of Plasmodium represents the count of the asexual parasites and is expressed as a number per $\mu 1$ blood or as a percentage of red blood cells infected. According to standard practice at the clinical laboratory (CLKB) from the Institute of Tropical Medicine (ITM), both thick and thin blood films are prepared, stained with Giemsa $(\mathrm{pH}$ 8.0) and examined by light microscopy using a $\times 500$ magnification. An examination of 15 minutes for a thick film with a minimum of 200 fields read, is performed before a thick blood film is reported negative. Parasite densities are estimated by counting asexual parasites against 200 white blood cells (WBC) in thick blood films and converting this number to parasites/ $\mu$ l using the actual WBC count or, when this is not available, the standard $8,000 \mathrm{WBC} / \mu 1$ value $[9,10]$.

Apart from not missing the diagnosis of malaria, differentiation of $P$. falciparum from the non-falciparum species is important, in particular not missing the diagnosis of $P$. falciparum because of the life-threatening potential of this species. Accurate identification of parasite density appears to be difficult in non-endemic settings [11], but high parasite densities exceeding $2 \%$ of red blood cells infected should be 
recognized, as this criterion constitutes an alert sign [7]. Expert microscopy should also recognize $P$. falciparum stages and hemozoin pigment in WBC: schizonts of $P$. falciparum in the peripheral circulation as well as hemozoin in the WBC in case of $P$. falciparum infection are indicators of a serious infection, whereas the exclusive presence of $P$. falciparum gametocytes after treatment is a normal finding $[7,12]$.

\section{Malaria rapid diagnostic tests can be of help in the laboratory diagnosis of malaria}

Microscopic diagnosis of malaria requires considerable training and experience. In resource-poor endemic settings, there may be problems related to equipment, expertise and workload, whereas in non-endemic settings in industrialized countries, there may be a lack of routine among the laboratory staff, resulting in low expertise [13-15].

Rapid diagnostic tests offer a simple and rapid complement to microscopic malaria diagnosis. They are of considerable help in the diagnosis of malaria and the identification of $P$. falciparum. However, they do not distinguish between $P$. falciparum and mixed species infections and do not give an estimation of parasite density $[8,14,16,17]$.

\section{What are Malaria Rapid Diagnostic Tests? How do they work?}

Malaria rapid diagnostic tests (RDTs) detect Plasmodium parasites in blood by an antibody-antigen reaction on a nitrocellulose strip [18]. This strip can be available as a self-standing strip or be embedded in a plastic cassette or occasionally in a cardboard format. Reactions on the nitrocellulose strip are visible as cherry-red lines. Two-band malaria RDTs are mostly designed to detect Plasmodium falciparum; they display a control line and a test line which targets either histidinerich protein-2 (HRP-2) or P. falciparum-specific parasite lactate dehydrogenase (PfpLDH). Three- and four-band malaria RDTs display a control line and two or three test lines, one targeting a $P$. falciparum specific antigen, another line targeting antigens common to the four species such as pan-Plasmodium-specific lactate parasite dehydrogenase (pan-pLDH) or aldolase, and, in case of the four band malaria RDTs, a third line which targets Plasmodium vivax-specific pLDH (PvpLDH).

HRP-2 $[19,20]$ is a protein produced by asexual stages and young gametocytes of $P$. falciparum. It is expressed on the red blood cell membrane surface and readily diffuses into the plasma. pLDH [18] is an enzyme in the glycolytic pathway of the Plasmodium spp., and is produced by sexual and asexual stages of the parasite. Aldolase is another enzyme of the Plasmodium glycolytic pathway that is also used as a target for detection. Of note is that the HRP-2 antigen can persist in the blood for up to several weeks after successful treatment (due to a low clearance), whereas 
pLDH and aldolase depend on living parasites and disappear from the circulation upon treatment [21].

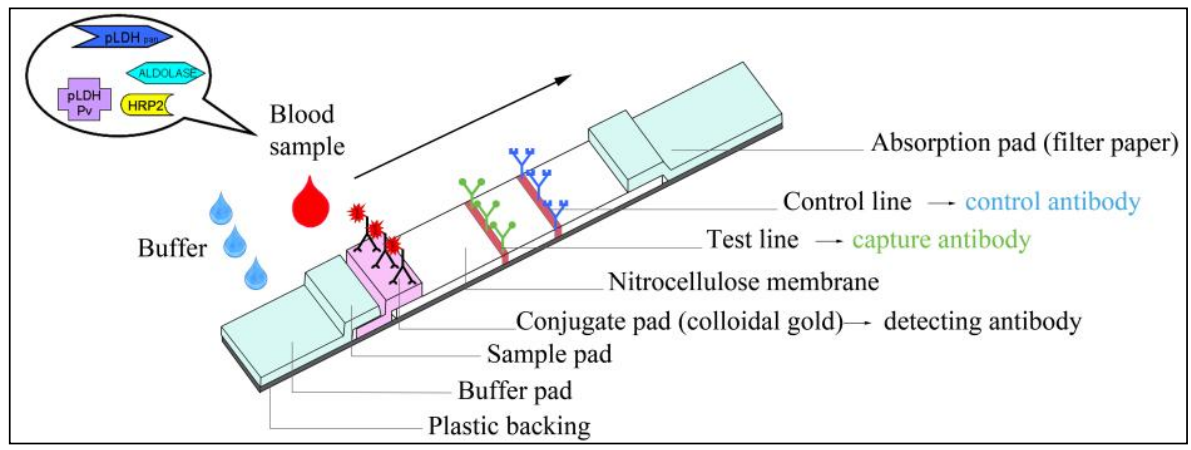

Figure 3: Schematic drawing of the malaria RDT lateral-flow strip.

Figures 3 and 4 show the different components of a malaria RDT strip and the sequence of reactions on a two-band malaria RDT strip respectively. The patient's blood and several drops of buffer (also called diluent) are applied respectively to the sample and buffer pad of the strip. They are attracted by the capillary action of an absorption pad at the other end of the strip and start to migrate. First, they pass the so-called conjugate pad, which contains a detection antibody targeting a Plasmodium antigen, such as HRP-2, Pf-pLDH, Pv-pLDH, pan-pLDH or aldolase. This detection antibody is a mouse-antibody that is conjugated to a signal, mostly colloidal gold. If present in the sample, the Plasmodium antigen is bound to this detection antibody-conjugate. Next, the antigen-antibody-conjugate complex migrates further across the strip until it is bound to a second antibody, the so-called capture antibody. This capture antibody binds to another epitope of the Plasmodium target antigen. As the capture antibody is applied on a narrow section of the strip, the complex with the conjugated signal will be concentrated and by virtue of the colloidal gold will become visible as a cherry-red coloured line. The excess of detection antibody-conjugate that was not bound by the antigen and the capture antibody moves further towards the absorption pad until it is bound to a goat-raised anti-mouse antibody, thereby generating a control line $[21,22]$. 

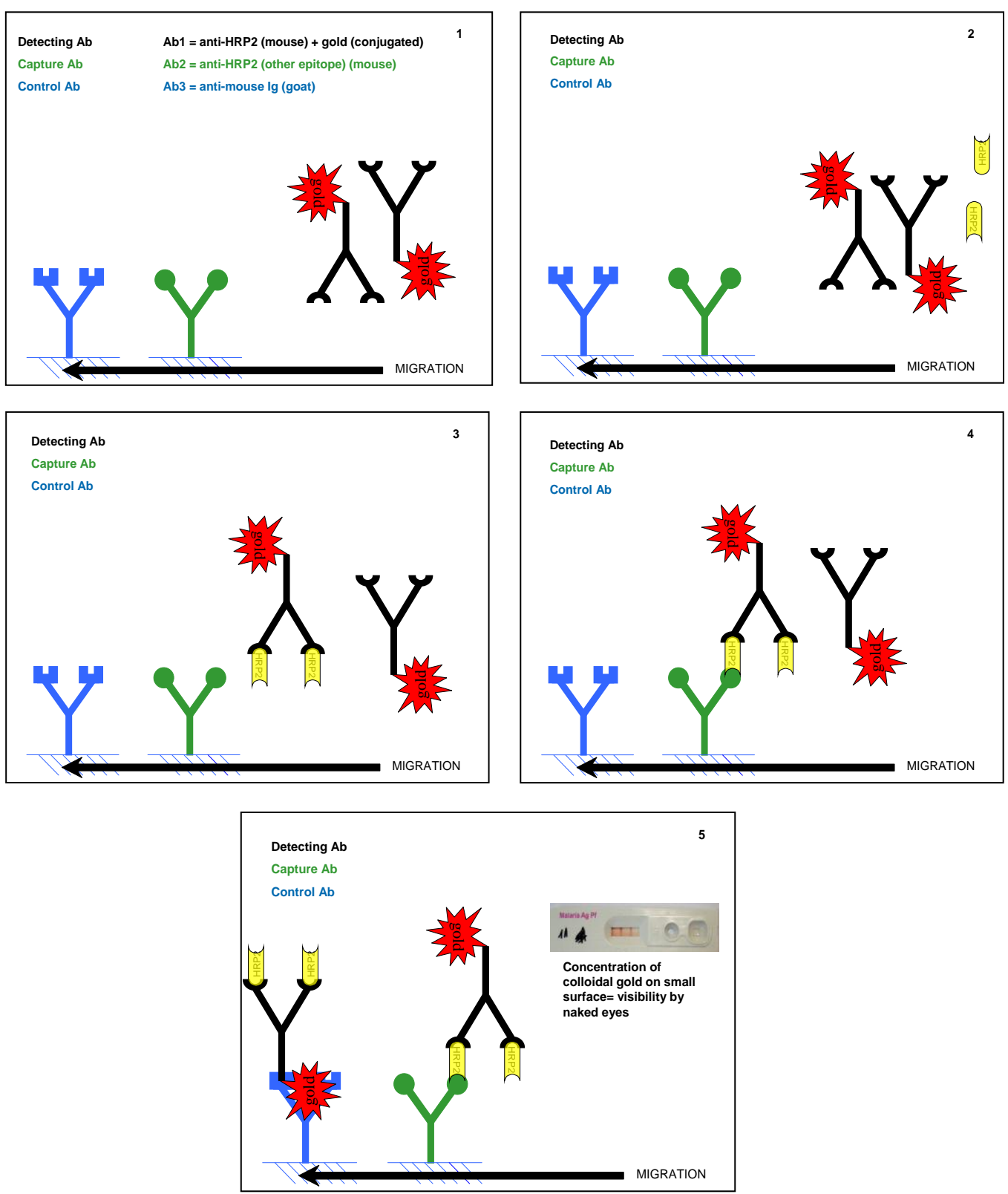

Figure 4: Two-band malaria RDT targeting HRP-2: consecutive reaction steps. 
Malaria RDTs use a simple but robust technique which has many other applications (HIV screening, pregnancy test, screening tests for drug use, rapid diagnostic tests for influenza, RSV etc). The technique as described above is frequently referred to as "immunochromatography", and malaria RDTs are also referred to as "lateral flow tests" as opposed to the "flow-through tests". In the former tests, the sample moves along the nitrocellulose strip whereas in the latter it moves through the membrane. An example of such a test system is the HIV spot, Genelabs Diagnostics, Singapore (one of the first rapid tests used for the detection of HIV antibodies). Lateral flow tests should neither be named "dipstick tests", because in dipstick tests there is no flow of the sample along the strip: the reaction is read at the place of the sample application. Due to their simple design and intrinsic robustness, malaria RDTs are heat-stable (until $30^{\circ} \mathrm{C}$ or even $45^{\circ} \mathrm{C}$ for some brands) and do not need a cold chain (in contrast to, for instance, latex agglutination tests). In addition, the technique allows for a long shelf-life (mostly more than 18 months). The colloidal gold conjugate however will be damaged at temperatures below $0^{\circ} \mathrm{C}$.

In malaria-endemic regions, malaria RDTs are currently rolled out by National Malaria Control Programs as a tool for parasite based diagnosis [23]. As they are simple to perform, they can also be carried out by non-specialized health care workers [24,25]. In non-endemic settings, where microscopic expertise is lacking due to low incidence, they are used as an adjunct to microscopy, especially outside opening hours but also as bedside point of care tests $[15,26]$, and they have been reported to perform accurately and even better as compared to microscopy $[27,28]$. In addition, malaria RDTs are marketed for self-use by travellers [29]. In 2007, more than 70.000.000 tests were performed [30] and more than 80 brands were marketed world-wide [23].

\section{Test characteristics of malaria RDTs for the detection of $\boldsymbol{P}$. falciparum}

Diagnostic performance of malaria RDTs available on the market are heterogeneous. Expressed in parasite count per $\mu 1$, some malaria RDTs have a detection limit of 100 asexual parasites (corresponding to $0.002 \%$ of red blood cells infected), and likewise comply with the WHO criteria of diagnostic sensitivity of at least $95 \%$ at this $100 / \mu 1$ threshold [31]. This detection limit generally is above the threshold of expert microscopy $(50 / \mu 1)$ but below that of routine microscopy in non-endemic settings,

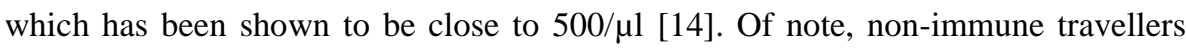
can present with symptoms at that parasite density [17].

Under controlled conditions, malaria RDTs have shown sensitivities close to $100 \%$ for the detection of $P$. falciparum, the most life-threatening species. However, as mentioned above, false negative results can occur at low parasite densities $(<100$ asexual parasites $/ \mu$ or $<0.002 \%$ of red blood cells parasitized). In such cases, the 
correct option is to repeat malaria RDT (and if possible microscopy) after $8-12$ hours, for up to four consecutive samplings [32,33]. Concerns have risen about rare but persistent reports of false negative malaria RDTs at higher parasite densities. Mostly, they are ascribed to polymorphisms of HRP-2 with the existence of variations that are less likely to be picked up by malaria RDTs. These variations are geographically confined to the Asia-Pacific and South-American regions [34-36]. The impact of these polymorphisms in the scope of travel medicine has not yet been studied.

False positive malaria RDTs are rare and there are no prospective data on their frequency. False positive results are particularly observed in patients with the rheumatoid factor and/or in patients with chronic infections, such as toxoplasmosis, hepatitis C, schistosomiasis, tuberculosis and syphilis [12,37].

\section{Observations leading to the research questions of this thesis}

Although malaria RDTs have been extensively evaluated for the diagnosis of $P$. falciparum malaria, their ability to detect the other Plasmodium species is less well documented. In addition, we noticed that despite big efforts on quality assurance at the level of production and distribution, there have been no formal external quality assessments organized for malaria RDTs. Finally, although simple, accurate and robust, malaria RDTs appear not to be fail-proof: in our daily practice of patientbased reference care, teaching and field work, we noticed shortcomings related to design and instructions of the malaria RDTs as well as to errors in performance and interpretation by the end-user. The present thesis focuses on these issues.

\section{Chapter II: Laboratory evaluation of malaria RDTs targeting $\boldsymbol{P}$. vivax}

Although there is a need for inexpensive rapid and accurate $P$. vivax-specific diagnostic tools for detecting single species and mixed $P$. vivax infections [38], malaria RDTs targeting $P$. vivax have been undergone limited evaluation $[8,17,39]$. In addition, due to differences in study design and population, it is difficult to compile all published findings. Most studies have investigated small numbers of the non-falciparum species, resulting in low precision but explaining in part for the wide range in reported sensitivities [16,17]. Only two malaria RDTs, Binax NOW [17,32,40-42] and OptiMAL [21,27,40,42-44] have been studied in detail. For Binax NOW in its most recent generation, compiled sensitivity for the diagnosis of $P$. vivax has been calculated to be $68.9 \%$ [17]. In a population of returned travellers, the Binax NOW displayed sensitivities of $86.7 \%$ for pure $P$. vivax samples with falsenegative results mainly but not exclusively found among samples with low parasite densities [32]. Similar values of sensitivity were obtained in endemic populations in Thailand and Columbia (87.3\% and $81.4 \%$ respectively) $[41,42]$. In comparison, the OptiMAL-IT which was run side-to-side in the latter study displayed a higher 
sensitivity for $P$. vivax $(91.0 \%)$, at the expense of a lower sensitivity for $P$. falciparum.

The SD FK70 Malaria Antigen Pv Test and the SD FK80 P.f/P.v Malaria Antigen Rapid Test (Standard Diagnostics, Hagal-Dong, Republic of Korea) are two recently released so-called one-step RDTs designed to detect $P$. vivax and both $P$. falciparum and $P$. vivax respectively. Chapter IIa describes the laboratory evaluation of the SD FK70 malaria Ag Plasmodium vivax rapid diagnostic test in a non-endemic setting and Chapter IIb describes the test characteristics of the SD FK80 Plasmodium falciparum/Plasmodium vivax malaria rapid diagnostic test in a non-endemic setting.

\section{Chapter III: Assessment of the prozone effect in malaria RDTs}

Low parasite density and mutations of the HRP-2 gene are mostly cited as the causes of false-negative malaria RDT results for $P$. falciparum [16,32,34-36,45].

The prozone effect may be an alternative explanation for false-negative results at high parasite densities. The prozone effect (also known as high dose hook phenomenon) is defined as false negative or false low results in immunological reactions, due to an excess of either antigens or antibodies. In these cases, high antigen concentrations will block all available binding sites of both the detection and the capture antibodies, thereby hindering binding of the antigen-detection antibodyconjugate complex to the capture antibody, with failure of signal generation (Figure 5).

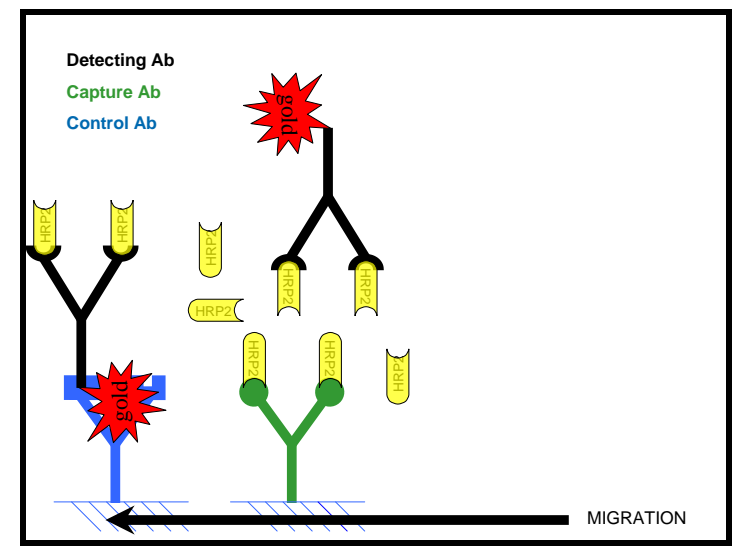

Figure 5: Prozone effect: antigen excess (in this case HRP-2) blocks all available binding sites, thereby preventing the binding between the colloidal gold- conjugated detection antibody (black) and the capture antibody (green).

Although cited by many leading documents, there is only a single original case report [29]. A clinical case of prozone submitted by a referring laboratory draw our 
attention to this phenomenon. In Chapter III, we studied the occurrence of prozone among a panel of malaria RDTs with different antigen targets in the laboratory (Chapter IIIa: assessment of the prozone effect in malaria rapid diagnostic tests) and next conducted a prospective field study to assess frequency and impact of prozone on clinical diagnosis in an endemic setting (Chapter IIIb: prozone in malaria rapid diagnostic test: how many cases are missed?).

\section{Chapter IV: Buffer substitution in malaria rapid diagnostic tests causes false- positive results}

By accident, we came across the phenomenon of false-positive malaria RDT results upon buffer substitution: during a practical teaching session we intended to demonstrate that the malaria RDT control line by itself only testifies correct migration but not correct performance of the malaria RDT test. Usually, students are asked to run a malaria RDT with buffer and with or without a sample. As the buffer vial was empty, we replaced the buffer with distilled water and run two HRP-2 malaria RDTs, one with water alone (without a sample) and one with water and blood of a malaria-free subject. We were all astonished to observe, apart from the expected control line, also a visible HRP-2 line on both malaria RDTs. The subject had no symptoms of malaria, nor did he suffer from malaria in the recent past. When performing the malaria RDT with the kit's dedicated buffer, there was no HRP-2 line visible. The HRP-2 line appeared upon retesting with distilled water and also when using saline and tap water as substitute liquid. We concluded a false-positive effect caused by buffer substitution.

During field visits (in particular in Africa), we repeatedly observed availability problems of the buffer vial. For instance, some cassettes were sent for testing in the ward, but the buffer vial did not return (Figure 6). To compensate, lab technicians took either a buffer vial from another kit (sometimes a kit of another brand), or used saline, distilled water (liquids for parenteral drug dilution) and occasionally tap water as substitute liquids. Apparently, this substitution of buffer seemed not to cause too much interference. We decided to explore this phenomenon and conducted a laboratory-based study described in Chapter IV. 


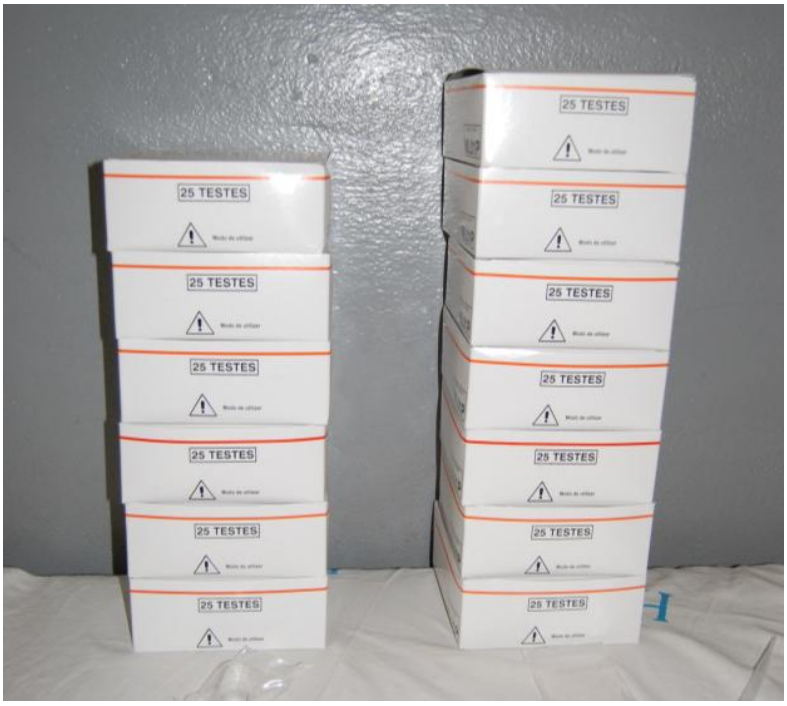

Figure 6: Example of buffer's availability for malaria RDTs in Mozambique (2009): in this laboratory, 13 kits of 25 tests were in stock. The six kits at the left of the picture were unopened and contained all materials needed for 25 tests (including the original buffer vial). The seven kits at the right, contained all the items needed, except the buffer vials that were already used with other kits.

\section{Chapter V: External quality assessment on the use of malaria rapid diagnostic tests in a non-endemic setting}

In recent years, initiatives for quality assurance of malaria RDTs along the route from design to end-user have been inspired by the WHO and the Foundation for Innovative New Diagnostics (FIND). These initiatives included laboratory evaluation rounds and a comprehensive quality control strategy for production, transport and product control and lot testing programs [46-49]. With regard to the end-user's performance, there has been the redaction of generic job aids [50,51] and the development of stable positive controls [52]. External quality assessment (EQA) sessions on the use of malaria RDTs had not yet been organized. In external quality assessment sessions, unknown samples are sent out by a reference laboratory to the participating laboratories, who assess the samples and return their reports. The reference laboratory compiles the results and gives a feedback to the participants including a didactic report that provides an educational stimulus to the participants. Apart from boosting the self-confidence of the participants, such EQA also provide insight into the participants' current level of performance, monitors changes in testing practices and may trace problems in test kits.

Chapter $\mathrm{V}$ describes the design, validation and results of an external quality assessment on the use of malaria RDTs in a non-endemic setting. 
Chapter VI: Malaria rapid diagnostic kits: quality of packaging, design and labelling of boxes and components and readability and accuracy of information inserts.

The results of the external quality assessment on malaria RDTs described in Chapter $\mathrm{V}$ pointed us to errors in interpretation of the results that were embedded in the information inserts supplied with the malaria RDT kits. Similar observations were made during the laboratory evaluation studies of malaria RDTs at CLKB: for instance, two malaria RDTs ordered in the scope of a study on P. falciparum/P. vivax cross-reaction claimed to be $P$. vivax-specific by their label and name but proved to detect pan-pLDH according to their package insert and two versions of a single kit (designed for laboratory and self-use respectively) showed discrepant instructions for test line interpretations [53,54]. In addition, during field visits in Africa, we occasionally noted shortcomings in malaria RDT kit boxes, content and instructions. Inspection of these information inserts also revealed a large variety in layout and readability, as well as variations in the adequacy of labelling of malaria RDT boxes and devices.

In Chapter VI, we assessed malaria RDT kits for adequate and correct design, construction and labelling of boxes and components, and the readability and accuracy of their information inserts.

Chapter VII provides a general discussion and introduces future perspectives. 
References

1. World Health Organization: World malaria report 2010. 2010. [http://whqlibdoc.who.int/publications/2010/9789241564106 eng.pdf]

2. Wéry M: Protozoologie médicale. De Boeck Université, 1995

3. World Health Organization: International travel and health. 2010. [http://www.who.int/entity/ith/ITH2010.pdf ]

4. Habib AG, Singh KS: Respiratory distress in nonimmune adults with imported malaria. Infection 2004, 32: 356-359.

5. Rogerson SJ, Carter R: Severe vivax malaria: newly recognised or rediscovered. PLoS Med 2008, 5: e136.

6. Phimpraphi W, Paul R, Witoonpanich B, Turbpaiboon C, Peerapittayamongkol C, Louicharoen C, Casademont I, Tungpradabkul S, Krudsood S, Kaewkunwal J, Sura T, Looareesuwan S, Singhasivanon P, Sakuntabhai A: Heritability of P. falciparum and P. vivax malaria in a Karen population in Thailand. PLOS ONE 2008, 3: e3887.

7. World Health Organization: Guidelines for the treatment of malaria, second edition, 2010. [http://whqlibdoc.who.int/publications/2010/9789241547925_eng.pdf ]

8. Wongsrichanalai C, Barcus MJ, Muth S, Sutamihardja A, Wernsdorfer WH: A review of malaria diagnostic tools: microscopy and rapid diagnostic test (RDT). Am J Trop Med Hyg 2007, 77: 119127.

9. World Health Organization: Basic Malaria Microscopy - Part II.Tutor's Guide 2nd edition, 2010. [www.searo.who.int/LinkFiles/Malaria_malaria_microscopy_Tutors_guide2010.pdf]

10. World Health Organization: Basic Malaria Microscopy - Part I. Learner's Guide 2nd edition, 2010. [www.searo.who.int/LinkFiles/Malaria_malaria_microscopy_Learners_guide2010.pdf]

11. Kettelhut MM, Chiodini PL, Edwards H, Moody A: External quality assessment schemes raise standards: evidence from the UKNEQAS parasitology subschemes. J Clin Pathol 2003, 56: $927-$ 932.

12. Grobusch MP, Hanscheid T, Gobels K, Slevogt H, Zoller T, Rogler G, Teichmann D: Comparison of three antigen detection tests for diagnosis and follow-up of falciparum malaria in travellers returning to Berlin, Germany. Parasitol Res 2003, 89: 354-357.

13. Hanscheid T: Current strategies to avoid misdiagnosis of malaria. Clin Microbiol Infect 2003, 9: 497-504.

14. Moody A: Rapid diagnostic tests for malaria parasites. Clin Microbiol Rev 2002, 15: 66-78.

15. Wiese L, Bruun B, Baek L, Friis-Moller A, Gahrn-Hansen B, Hansen J, Heltberg O, Hojbjerg T, Hornstrup MK, Kvinesdal B, Gomme G, Kurtzhals JA: Bedside diagnosis of imported malaria using the Binax Now malaria antigen detection test. Scand J Infect Dis 2006, 38: 1063-1068.

16. Marx A, Pewsner D, Egger M, Nuesch R, Bucher HC, Genton B, Hatz C, Juni P: Meta-analysis: accuracy of rapid tests for malaria in travelers returning from endemic areas. Ann Intern Med 2005, 142: 836-846.

17. Murray CK, Gasser RA, Jr., Magill AJ, Miller RS: Update on Rapid Diagnostic Testing for Malaria. Clin Microbiol Rev 2008, 21: 97-110.

18. Shiff CJ, Premji Z, Minjas JN: The rapid manual ParaSight-F test. A new diagnostic tool for Plasmodium falciparum infection. Trans R Soc Trop Med Hyg 1993, 87: 646-648.

19. Howard RJ, Uni S, Aikawa M, Aley SB, Leech JH, Lew AM, Wellems TE, Rener J, Taylor DW: Secretion of a malarial histidine-rich protein (Pf HRP II) from Plasmodium falciparuminfected erythrocytes. $J$ Cell Biol 1986, 103: 1269-1277.

20. Rock EP, Marsh K, Saul AJ, Wellems TE, Taylor DW, Maloy WL, Howard RJ: Comparative analysis of the Plasmodium falciparum histidine-rich proteins HRP-I, HRP-II and HRP-III in malaria parasites of diverse origin. Parasitology 1987, 95 ( Pt 2): 209-227.

21. Moody AH, Chiodini PL: Non-microscopic method for malaria diagnosis using OptiMAL IT, a second-generation dipstick for malaria pLDH antigen detection. Br J Biomed Sci 2002, 59: 228231.

22. Bell D, Peeling RW: Evaluation of rapid diagnostic tests: malaria. Nat Rev Microbiol 2006, 4: S34-S38.

23. Drakeley $\mathrm{C}$, Reyburn $\mathrm{H}$ : Out with the old, in with the new: the utility of rapid diagnostic tests for malaria diagnosis in Africa. Trans R Soc Trop Med Hyg 2009, 103: 333-337.

24. Mayxay M, Newton PN, Yeung S, Pongvongsa T, Phompida S, Phetsouvanh R, White NJ: Short communication: An assessment of the use of malaria rapid tests by village health volunteers in rural Laos. Trop Med Int Health 2004, 9: 325-329. 
25. Rennie W, Phetsouvanh R, Lupisan S, Vanisaveth V, Hongvanthong B, Phompida S, Alday P, Fulache M, Lumagui R, Jorgensen $\mathrm{P}$, Bell D, Harvey S: Minimising human error in malaria rapid diagnosis: clarity of written instructions and health worker performance. Trans $R$ Soc Trop Med Hyg 2007, 101: 9-18.

26. Chilton D, Malik AN, Armstrong M, Kettelhut M, Parker-Williams J, Chiodini PL: Use of rapid diagnostic tests for diagnosis of malaria in the UK. J Clin Pathol 2006, 59: 862-866.

27. Palmer CJ, Bonilla JA, Bruckner DA, Barnett ED, Miller NS, Haseeb MA, Masci JR, Stauffer WM: Multicenter study to evaluate the OptiMAL test for rapid diagnosis of malaria in U.S. hospitals. J Clin Microbiol 2003, 41: 5178-5182.

28. Stauffer WM, Cartwright CP, Olson DA, Juni BA, Taylor CM, Bowers SH, Hanson KL, Rosenblatt JE, Boulware DR: Diagnostic performance of rapid diagnostic tests versus blood smears for malaria in US clinical practice. Clin Infect Dis 2009, 49: 908-913.

29. Risch L, Bader M, Huber AR: [False negative quick malaria test]. Schweiz Med Wochenschr 1999, 129: 1002.

30. Bell D, Perkins MD: Making malaria testing relevant: beyond test purchase. Trans $R$ Soc Trop Med Hyg 2008, 102: 1064-1066.

31. World Health Organization: Regional Office for the Western Pacific 2003. Malaria Rapid Diagnosis: Making it Work. Meeting report 20-23 January 2003. Manila, the Philippines. 2003. [http://www.searo.who.int/LinkFiles/Malaria_MalariaRDT.pdf]

32. Farcas GA, Zhong KJ, Lovegrove FE, Graham CM, Kain KC: Evaluation of the Binax NOW ${ }^{\circledR}$ ICT test versus polymerase chain reaction and microscopy for the detection of malaria in returned travelers. Am J Trop Med Hyg 2003, 69: 589-592.

33. Forney JR, Magill AJ, Wongsrichanalai C, Sirichaisinthop J, Bautista CT, Heppner DG, Miller RS, Ockenhouse CF, Gubanov A, Shafer R, DeWitt CC, Quino-Ascurra HA, Kester KE, Kain KC, Walsh DS, Ballou WR, Gasser RA, Jr.: Malaria rapid diagnostic devices: performance characteristics of the ParaSight F device determined in a multisite field study. J Clin Microbiol 2001, 39: 28842890 .

34. Baker J, McCarthy J, Gatton M, Kyle DE, Belizario V, Luchavez J, Bell D, Cheng Q: Genetic Diversity of Plasmodium falciparum Histidine-Rich Protein 2 (PfHRP2) and Its Effect on the Performance of PfHRP2-Based Rapid Diagnostic Tests. J Infect Dis 2005, 192: 870-877.

35. Baker J, Ho MF, Pelecanos A, Gatton M, Chen N, Abdullah S, Albertini A, Ariey F, Barnwell J, Bell D, Cunningham J, Djalle D, Echeverry DF, Gamboa D, Hii J, Kyaw MP, Luchavez J, Membi C, Menard D, Murillo C, Nhem S, Ogutu B, Onyor P, Oyibo W, Wang SQ, McCarthy J, Cheng Q: Global sequence variation in the histidine-rich proteins 2 and 3 of Plasmodium falciparum: implications for the performance of malaria rapid diagnostic tests. Malar J 2010, 9: 129.

36. Gamboa D, Ho MF, Bendezu J, Torres K, Chiodini PL, Barnwell JW, Incardona S, Perkins M, Bell $\mathrm{D}$, McCarthy J, Cheng Q: A large proportion of $\mathbf{P}$. falciparum isolates in the Amazon region of Peru lack pfhrp2 and pfhrp3: implications for malaria rapid diagnostic tests. PLoS One 2010, 5: e8091.

37. Iqbal J, Sher A, Rab A: Plasmodium falciparum histidine-rich protein 2-based immunocapture diagnostic assay for malaria: cross-reactivity with rheumatoid factors. J Clin Microbiol 2000, 38: $1184-1186$.

38. Sina B: Focus on Plasmodium vivax. Trends Parasitol 2002, 18: 287-289.

39. Lee SW, Jeon K, Jeon BR, Park I: Rapid diagnosis of vivax malaria by the SD Bioline Malaria Antigen test when thrombocytopenia is present. J Clin Microbiol 2008, 46: 939-942.

40. Playford EG, Walker J: Evaluation of the ICT malaria P.f/P.v and the OptiMal rapid diagnostic tests for malaria in febrile returned travellers. J Clin Microbiol 2002, 40: 4166-4171.

41. van den Broek I, Hill O, Gordillo F, Angarita B, Hamade P, Counihan H, Guthmann JP: Evaluation of three rapid tests for diagnosis of $\mathbf{P}$. falciparum and $\mathbf{P}$. vivax malaria in Colombia. Am $J$ Trop Med Hyg 2006, 75: 1209-1215.

42. Wongsrichanalai C, Arevalo I, Laoboonchai A, Yingyuen K, Miller RS, Magill AJ, Forney JR, Gasser RA, Jr.: Rapid diagnostic devices for malaria: field evaluation of a new prototype immunochromatographic assay for the detection of Plasmodium falciparum and nonfalciparum Plasmodium. Am J Trop Med Hyg 2003, 69: 26-30.

43. Coleman RE, Maneechai N, Rachapaew N, Kumpitak C, Soyseng V, Miller RS, Thimasarn K, Sattabongkot J: Field evaluation of the ICT Malaria Pf/Pv immunochromatographic test for the detection of asymptomatic malaria in a Plasmodium falciparum/vivax endemic area in Thailand. Am J Trop Med Hyg 2002, 66: 379-383. 
44. Iqbal J, Hira PR, Sher A, Al Enezi AA: Diagnosis of imported malaria by Plasmodium lactate dehydrogenase (pLDH) and histidine-rich protein 2 (PfHRP-2)-based immunocapture assays. Am J Trop Med Hyg 2001, 64: 20-23.

45. Erdman LK, Kain KC: Molecular diagnostic and surveillance tools for global malaria control. Travel Med Infect Dis 2008, 6: 82-99.

46. World Health Organization: Malaria Rapid Diagnostic Test Performance; Results of WHO product testing of malaria RDTs: Round 1 (2008). 2009.

[http://www.finddiagnostics.org/resource-centre/reports brochures/malaria-diagnostics-report2009.html]

47. World Health Organization: Lot-testing: Using quality control parasite dilutions to test the sensitivity of malaria RDTs. 2010.

[http://www.wpro.who.int/sites/rdt/using_rdts/qa/lot_testing.htm ]

48. World Health Organization: Overview of the prequalification of diagnistics assessment process. 2010.

[http://www.who.int/entity/diagnostics_laboratory/evaluations/pqdx_007_pq_overview_document_v 3.pdf]

49. World Health Organization: Malaria Rapid Diagnostic Test Performance; Results of WHO product testing of malaria RDTs: Round 2 (2009). 2010.

[http://www.finddiagnostics.org/resource-centre/reports_brochures/malaria-diagnostic-test-reportround2.html ]

50. World Health Organization: How to use a Rapid Diagnostic Test (RDT): A guide for training at a village and clinic level. 2008.

[http://www.wpro.who.int/sites/rdt/using rdts/training/rdt training falciparum.htm]

51. World Health Organization: RDT Instructions and Training. 2008. [www.webcitation.org/query.php?url=http://www.wpro.who.int/sites/rdt/using rdts/training/rdt train ing falciparum.htm\&refdoi=10.1186/1475-2875-9-359]

52. Versteeg I, Mens PF: Development of a stable positive control to be used for quality assurance of rapid diagnostic tests for malaria. Diagn Microbiol Infect Dis 2009, 64: 256-260.

53. Maltha J, Gillet P, Bottieau E, Cnops L, Van Esbroeck M, Jacobs J: Evaluation of a rapid diagnostic test (CareStart Malaria HRP-2/pLDH (Pf/pan) Combo Test) for the diagnosis of malaria in a reference setting. Malar J 2010, 9: 171.

54. Maltha J, Gillet P, Cnops L, Van Den Ende J, Van Esbroeck M, Jacobs J: Malaria rapid diagnostic tests: Plasmodium falciparum infections with high parasite densities may generate false positive Plasmodium vivax pLDH lines. Malar J 2010, 9: 198. 


\section{Chapter II}

\section{Laboratory evaluation of malaria RDTs targeting $P$. vivax}

IIa: Laboratory evaluation of the SD FK70 malaria Ag Plasmodium vivax rapid diagnostic test in a non-endemic setting.

Gillet P, Bosselaers K, Cnops L, Bottieau E, Van

Esbroeck M, Jacobs J. Malaria Journal 2009; 8:129.

http://www.malariajournal.com/content/8/1/129

IIb: Test characteristics of the SD FK80 Plasmodium falciparum/Plasmodium vivax malaria rapid diagnostic test in a non-endemic setting.

Gillet P, van Dijk DP, Bottieau E, Cnops L, Van Esbroeck M, Jacobs J. Malaria Journal 2009; 8:262. http://www.malariajournal.com/content/8/1/262 


\section{Chapter IIa}

\section{Laboratory evaluation of malaria RDTs}

targeting $P$. vivax

IIa: Laboratory evaluation of the SD FK70 malaria Ag

Plasmodium vivax rapid diagnostic test in a non-endemic setting.

Gillet P, Bosselaers K, Cnops L, Bottieau E, Van Esbroeck M, Jacobs J.

Malaria Journal 2009; 8:129.

http://www.malariajournal.com/content/8/1/129 


\title{
Evaluation of the SD FK70 Malaria Ag Plasmodium vivax rapid diagnostic test in a non-endemic setting
}

\begin{abstract}
Background

For clinical and epidemiological reasons, it is interesting to diagnose non-falciparum malaria to the species level. This retrospective study assessed the performance of the SD BIOLINE Malaria Antigen Pv test (FK70), a two-band immunochromatographic test detecting Plasmodium vivax-specific lactate dehydrogenase, on samples of international travellers in a non-endemic setting.
\end{abstract}

\section{Methods}

Stored blood samples from international travellers suspected of malaria were used, with microscopy corrected by PCR as the reference method. Samples infected by Plasmodium vivax $(\mathrm{n}=100)$, Plasmodium falciparum $(\mathrm{n}=75)$, Plasmodium ovale $(\mathrm{n}$ $=75)$ and Plasmodium malariae $(\mathrm{n}=25)$ were included, as well as 100 malarianegative samples. End points were sensitivity, specificity, inter-reader reliability and reproducibility.

\section{Results}

The overall sensitivity of the FK70 for the diagnosis of $P$. vivax was $88.0 \%$ (95\% confidence interval (CI): $83.6 \%$ - 90.3\%). For parasite densities $>500 / \mu 1$, a sensitivity of $97.2 \%$ (CI: $92.6 \%-99.1 \%$ ) was obtained. Specificity was $98.5 \%$, with 4 out of $75 P$. falciparum samples testing positive. None of the $P$. ovale samples tested positive. Nearly two-thirds $(57 / 88,64.7 \%)$ of positive $P$. vivax samples showed faint or weak line intensities, with stronger line intensities at higher parasite densities. The test showed excellent reproducibility and reliability for test results and line intensities (kappa values exceeding 0.98 and 0.87 respectively).

\section{Conclusions}

The FK70 test performed well in diagnosing $P$. vivax infections in a non-endemic reference setting. It can be of added value to microscopy in species differentiation of malaria infections, especially at parasite densities $>500 / \mu 1$. 


\section{Background}

Malaria is a condition that requires prompt diagnosis, for which microscopy is considered as the reference standard. Although cheap, reliable and available on an instant base, microscopy has its limitations. For instance, in resource-limited centres, there are problems of equipment, training and workload, whereas in non-endemic countries, laboratory staff may lack sufficient exposure to malaria positive samples resulting in low expertise [1,2]. Malaria rapid diagnostic tests (RDTs) have proven to be a valuable adjunct to microscopy. The tests initially developed included a test control line and a Plasmodium falciparum-specific line targeting histidine-rich protein-2 (HRP-2) or P. falciparum-specific parasite lactate dehydrogenase (pLDH). These so-called two-band tests only detect $P$. falciparum. The three-band test, engineered later, comprises a third line for detection of an antigen common to the four Plasmodium species, such as pan Plasmodium-specific pLDH or aldolase. These RDTs still only distinguish between infections due to $P$. falciparum (or a mix of $P$. falciparum and one or more of the non-falciparum species) on the one hand, and infections with any of the non-falciparum species on the other hand. Although monoclonal antibodies directed to Plasmodium vivax-specific $\mathrm{pLDH}(\mathrm{Pv}-\mathrm{pLDH})$ have been developed, RDTs addressing this target have undergone only limited evaluation $[3,4]$.

The SD FK70 Malaria Antigen Pv test (05-FK70-02-0, Standard Diagnostics, HagalDong, Republic of Korea), further referred to as FK70, is a two-band RDT that targets $P$. vivax-specific pLDH. This test was evaluated as part of an accreditation process in the laboratory.

\section{Methods}

\section{Study design}

In this retrospective study, the FK70 was evaluated against a collection of stored samples obtained from international travellers. Tests were carried out in the reference laboratory of the Institute of Tropical Medicine (ITM) Antwerp, Belgium.

\section{Patients and samples}

Samples were selected from a collection of EDTA-blood samples stored at $-70^{\circ} \mathrm{C}$ and obtained from patients presenting at the outpatient clinic of ITM. The patients were international travellers and, to a lesser extent, immigrants returning from visits to their native countries. In addition, samples sent by Belgian laboratories to ITM in the scope of the national reference function were included. A representative number of samples were selected $(n=375)$, including the four malaria species with varying parasite densities and representative geographic distribution. 


\section{Reference method}

All samples were analysed by microscopy and real-time polymerase chain reaction (PCR). In case of discordant results, microscopy was corrected by PCR as the reference method. Standard microscopy was performed on thick blood films of all samples to diagnose malaria and to assess parasite density, and on thin blood films of positive samples to define the Plasmodium species. Thick blood films were stained with Giemsa 3.5\% (Merck, KGmA, Darmstadt, Germany) $(\mathrm{pH}=8.0$ ) for 20 minutes, thin films with May-Grünwald Giemsa. The slides were examined by light microscopy using a $\times 500$ magnification, according to the standard procedure at ITM. Parasite density was assessed by counting the number of asexual parasites against 200 white blood cells in a thick film, converting this to parasites/ $\mu 1$ using the actual count or the standard of 8,000 white blood cell/ $\mu 1$ [2].

Real-time PCR analysis was adapted from Rougemont et al [5] and had been validated against as part of ISO15189 accreditation. DNA of all 375 samples was extracted with a QIAamp DNA minikit (Qiagen Benelux, Venlo, The Netherlands) using the same set of primers and species-specific probes (Biolegio, Nijmegen, the Netherlands), with reporters and quenchers adapted to the dye channels of the Cepheid Smart Cycler II device (Lucron Bioproducts, De Pinte, Belgium). Two PCR reactions were run in parallel, a duplex reaction to detect $P$. falciparum and $P$. vivax and another reaction for $P$. ovale and $P$. malariae. In the first reaction, the $25 \mu 1$ reaction mix contained $5 \mu$ l DNA, 1× Quantitec mix (Qiagen Benelux), 200nM forward and reverse primer, 100nM falciparum probe and 200nM vivax probe. In the second reaction, besides $5 \mu \mathrm{l}$ template DNA and 1x Quantitec mix (Qiagen Benelux), 500nM forward and reverse primer, 320nM ovale probe, 200nM malariae probe and $1 \mathrm{mM} \mathrm{MgCl}_{2}$ were added. The PCR programme consisted of an initial step of $15 \mathrm{~min}$ at $95^{\circ} \mathrm{C}$ followed by 50 cycles of $5 \mathrm{sec}$ at $95^{\circ} \mathrm{C}, 30 \mathrm{sec}$ at $58^{\circ} \mathrm{C}$ and finally $30 \mathrm{sec}$ at $72^{\circ} \mathrm{C}$.

\section{Test platform}

The FK70 is a lateral flow immunochromatographic RDT in a cassette format. Two lines are present, a control line, which indicates whether the test is valid, and a PvpLDH line. According to the manufacturer's instructions, any visible Pv-pLDH line should be considered as positive, and results are expressed as positive or negative for $P$. vivax.

For the evaluation, test kits of two different lot numbers were used, RDT7001 and BD7001 with expiry dates of 12.08 .09 and 27.08.09 respectively.

\section{Test procedure}

Tests were performed according to the instructions of the manufacturer, except that samples $(5 \mu \mathrm{l})$ were loaded with a transfer pipette (Finnpippette, Helsinki, Finland) 
instead of the plastic loop supplied by the manufacturer and that a scoring system was used to assess the intensity of the Pv-pLDH line. In case the control line did not appear, the result was interpreted as invalid and the test was repeated. In order to score Pv-pLDH line intensities, the scoring system of Bell and co-workers [6] was applied and defined five categories: none (no line visible), faint (barely visible line), weak (paler than the control line), medium (equal to the control line) or strong (stronger than the control line). To assure timely readings, tests were carried out in time-controlled batches of ten samples. Readings were performed by three subsequent readers, of whom the one who performed the test procedure invariably was the first. Readers were blinded to the results of microscopy and to each others' readings. Readings were carried out at daylight assisted by a standard electricity bulb, between 20 and 30 minutes (but not beyond) after application of the sample and buffer. The results of the readings considered were based on consensus agreement, which means that the same result was observed by at least two out of three different readers. When there was no consensus, results of the first reader were considered. Inter-reader reliabilities were assessed for the test results expressed as positive and negative readings as well as for the Pv-pLDH line intensity readings. To assess reproducibility, a panel of 25 samples (including $20 P$. vivax samples, four $P$. falciparum samples and one $P$. ovale sample) was tested on three successive occasions.

\section{Statistical analysis}

True positive results were defined as those with a Pv-pLDH line visible in samples with $P$. vivax seen at microscopy, and true negative results as those with no $\mathrm{Pv}$ pLDH line visible in microscopy-negative samples and in samples with other Plasmodium species. False-negative samples were identified as those with a microscopic diagnosis of $P$. vivax, but no Pv-pLDH line visible, and false-positive samples as microscopic negative samples and samples with non-vivax Plasmodium showing a Pv-pLDH line.

From these categories sensitivity and specificity were calculated with $95 \%$ confidence intervals (C.I.). Reliabilities for positive and negative readings and line intensities were calculated as percentage agreements for all three readers and kappa values for each pair of readers. Differences between proportions were tested for significance using the chi-square test or, in case of small sample sizes, a two-tailed Fisher's exact test. A p-value $<0.05$ was considered as significant. Associations between line intensity readings and parasite densities were assessed for strength of association with Cramer's $V$ for categorical variables.

\section{Ease of use}

Three experienced laboratory technicians scored the ease of use of the FK70 test and the clarity of manufacturer's instructions with a standardized list. 


\section{Ethical review}

The study was reviewed and approved by the Institutional Review Board of ITM and by the Ethical Committee of Antwerp University, Belgium.

\section{Results}

\section{Sample collection}

Out of 1,324 stored samples of ITM, 375 samples were selected, of which 49 samples were sent by Belgian laboratories to ITM for second opinion. The samples were collected from December 1995 to November 2007. According to microscopy and after correction for PCR analysis, 100 of these samples were positive for $P$. vivax, 75 for $P$. ovale, 75 for $P$. falciparum and 25 for $P$. malariae. The results of microscopy were corrected in 11 out of 375 (2.9\%) samples and were uniquely related to $P$. vivax $-P$. ovale mismatches: 4 out of $75(5.3 \%)$ and 7 out of 100 (7.0\%) samples that had been categorised as $P$. vivax and $P$. ovale by the original microscopy were identified as $P$. ovale and $P$. vivax respectively by PCR. The microscopic identification of all $P$. falciparum, and $P$. malariae samples as well as the results of microscopy-negative samples were confirmed by PCR.

In addition, 100 microscopic and PCR negative samples of symptomatic travellers were included in the panel. The majority of the $P$. vivax samples had been acquired in Asia.

\section{Invalid test results}

Two of the 375 samples gave invalid results at initial testing. After application into the respective well, neither the blood nor the buffer started to migrate. Upon repetition, tests performed well.

\section{Sensitivity and specificity}

Table 1 lists test characteristics matched with species identification and parasite density. The sensitivity at parasite densities > 500/ $\mu$ l was $97.2 \%$ (Confidence Interval (CI): $92.6 \%-99.1 \%$ ) but was less at lower parasite densities < 500/ $\mu 1(64.3$ $\%, \mathrm{p}<0.001$ ), with 10 out of 12 false negative samples occurring in this category. Challenged with other Plasmodium species and negative samples, the FK70 showed a specificity of $98.5 \%$, with 4 out of 75 (5.3\%) P. falciparum samples showing cross-reactions. Of interest is that none out of $75 P$. ovale samples tested positive.

\section{Line intensities}

Table 2 lists the Pv-pLDH line intensities matched to species identification and parasite density. Nearly two-thirds $(57 / 88,64.7 \%)$ of all positive $P$. vivax samples generated faint or weak line intensities. These occurred not only at low parasite densities $(<500 / \mu 1)$, but also in 8/11 samples and 31/61 samples of parasite densities between $501-1,000 / \mu 1$ and $>1,000 / \mu 1$ respectively. Line intensity readings were 
significantly related to parasite densities with a substantial correlation (Cramer's $V=$ $0.5633, \mathrm{p}<0.001)$, but there was considerable overlap between categories.

Table 1: Test characteristics of the FK70Test characteristics of the FK70 matched with species and parasite density, PCR-corrected microscopy as the reference method $(\mathrm{n}=375)$.

\begin{tabular}{|c|c|c|c|c|}
\hline $\begin{array}{l}\text { Species and parasite density } \\
\quad(\text { asexual parasites/ } \mu \mathrm{l})\end{array}$ & $\begin{array}{l}\text { Numbers } \\
\text { tested }\end{array}$ & $\begin{array}{c}\text { Numbers tested } \\
\text { positive by } \\
\text { FK70 }\end{array}$ & $\begin{array}{l}\text { Sensitivity \% } \\
\quad(95 \% \text { CI })\end{array}$ & $\begin{array}{l}\text { Specificity \% } \\
\quad(95 \% \text { CI })\end{array}$ \\
\hline P. vivax, $0-500 / \mu 1$ & 28 & 18 & $\begin{array}{c}64.3 \\
(51.0-72.3)\end{array}$ & \\
\hline P. vivax, $501-1,000 / \mu 1$ & 11 & 11 & $\begin{array}{c}100.0 \\
(78.8-100.0)\end{array}$ & \\
\hline P. vivax, $>1,000 / \mu 1$ & 61 & 59 & $\begin{array}{c}96.7 \\
(91.3-99.0)\end{array}$ & \\
\hline P. vivax, all samples combined & 100 & 88 & $\begin{array}{c}88.0 \\
(83.6-90.3)\end{array}$ & \\
\hline Other Plasmodium species & 175 & 4 & & $\begin{array}{c}98.5 \\
(96.9-99.4)\end{array}$ \\
\hline P. falciparum & 75 & 4 & & \\
\hline P. ovale & 75 & 0 & & \\
\hline P. malariae & 25 & 0 & & \\
\hline Malaria negative samples & 100 & 0 & & \\
\hline
\end{tabular}

Table 2: Line intensities of the Pv-PLDH line FK70 line intensity of the Pv-pLDH line for the different parasite densities

\begin{tabular}{|c|c|c|c|c|c|c|}
\hline \multirow[b]{2}{*}{$\begin{array}{l}\text { Species and parasite } \\
\text { density }\end{array}$} & \multicolumn{6}{|c|}{ Consensus readings of $\mathrm{Pv}$-pLDH line* } \\
\hline & Negative & Faint & Weak & Medium & Strong & Total \\
\hline$P$. vivax $0-500 / \mu 1$ & 10 & 4 & 14 & & & 28 \\
\hline P. vivax $501-1,000 / \mu 1$ & & 1 & 7 & 2 & 1 & 11 \\
\hline P. vivax $>1,000 / \mu 1$ & 2 & 4 & 27 & 17 & 11 & 61 \\
\hline P. falciparum & 71 & & 4 & & & 75 \\
\hline P. ovale & 75 & & & & & 75 \\
\hline P. malariae & 25 & & & & & 25 \\
\hline Malaria negative samples & 100 & & & & & 100 \\
\hline Total & 283 & 9 & 52 & 19 & 12 & 375 \\
\hline
\end{tabular}

\footnotetext{
* Consensus Readings: line intensity as read by at least two out of three readers
} 


\section{Inter-reader reliability}

Inter-reader reliability for positive and negative test results was high, with $99.2 \%$ agreement between the three readers and kappa values exceeding 0.98 for each pair of readers. Two samples of $P$. vivax with parasite densities of 82 and 230/ $\mu 1$ were reported as negative by one reader while the other two readers reported them as faint; an additional sample with a parasite density of $10,556 / \mu 1$ was reported as faint by two readers and as negative by the third one. Reliability for Pv-pLDH line intensity readings was also high, with an overall agreement of $94.2 \%$ and kappa values between the different pairs of readers of $0.87,0.88$ and 0.92 respectively. For strong intensities, all readings were identical. Less consistent readings were obtained for other intensities, but discordances between two readers were always within one category of difference.

\section{Reproducibility}

Test results and Pv-pLDH line intensity readings were reproducible. Consistent line intensity readings for all readers upon three times repetition were obtained for 12 of the 25 samples. For 10 other samples, discordances occurred only within one category of difference in line intensity. The three remaining samples showed differences in more than one category of difference, with negative readings, faint and/or weak readings.

\section{Analysis of discordant results}

For sixteen samples, the FK70 and PCR-corrected microscopy provided discordant results. Twelve $P$. vivax samples tested negative and four $P$. falciparum samples tested positive. The false-negative $P$. vivax samples showed no particular geographic distribution and most of them had low parasite densities $(<500 / \mu 1)$ (Table 2). The four false positive $P$. falciparum samples showed weak line intensities; they were acquired in the Cameroon, Guinea, the Democratic Republic of the Congo, and Nigeria (the latter two in autochthonous native Africans) and had parasite densities of $7,000,158,000,264,000$ and $1,000,000 / \mu 1$, respectively. All samples gave identical results upon retesting except for a single $P$. falciparum sample (with parasite density of 7,000/ $\mu 1$ ) that was negative upon retesting. Real-time PCR for all four samples was conclusive for a single species $P$. falciparum infection. The samples represented 3/16 samples with parasite densities above 100,000/ $\mu$ l and 1/59 samples with parasite densities lower than $100,000 / \mu 1(\mathrm{p}<0.05)$.

\section{Ease of use}

The FK70 was scored as practical and easy in use and the instructions were scored as clear and simple to perform by all three technicians. 


\section{Discussion}

This retrospective study demonstrated that the two-band FK70 test performed well for the detection of $P$. vivax in a non-endemic reference setting. The FK70 was scored as easy-to-use and showed an overall sensitivity and specificity of $88.0 \%$ and $98.5 \%$ respectively, with excellent reliability and reproducibility.

Several limitations of this study have to be considered. Its retrospective design precluded exploration of discrepant results, and although the representative malaria negative control samples (i.e. samples of symptomatic international travellers), the test was not challenged with samples that are known to cause false positive results such as those presenting the rheumatoid factor [7]. Next, calibrated transfer pipette was used instead of the manufacturer's transfer device, thereby bypassing a possible error of the kit's application loop. An effect of storage of the samples on the test results cannot be excluded, but seems to be unlikely, as observed similar patterns of test results and Pv-pLDH line intensities on fresh samples analysed prospectively after introduction of the FK70 in the ITM malaria reference laboratory. Finally, it should be noted that the present findings apply to a non-endemic reference setting and should not be as such extrapolated to field settings: for instance, reported sensitivities of RDTs tend to be lower in field settings as compared to reference settings due to better test conditions in the latter [3,8]. Likewise, trained lab technicians generally tend to score tests and instructions more favourably than less trained end-users in remote settings, who may experience more practical difficulties when handling the RDTs [9].

It is difficult to compare the present findings to the reported literature. Although RDTs have been extensively evaluated for the diagnosis of $P$. falciparum malaria, their ability to detect the other Plasmodium species is less documented. Most studies have investigated small numbers of the non-falciparum species, resulting in low precisions and explaining in part for the wide range in reported sensitivities $[3,10]$. Adding to the difficulties are the evolutions in proprietary compositions and brand names of RDTs, with continuous releases of improved designs as well as wellstudied products withdrawn from the market [11]. The World Health Organization (WHO) lists RDT manufacturers and distributors online [12], but it is not clear from the list whether in case a particular RDT detects $P$. vivax by Pv-pLDH or by panPlasmodium-pLDH. According to the literature, the DBEST One Step Malaria Test (AmeriTek, Everett, WA, USA) and the FK70 are at present the only marketed twoband RDTs that specifically detect $P$. vivax. In general, sensitivities of three-band RDTs for the detection of $P$. vivax tend to be somewhat lower than presently described for the two-band FK70. As an example, compiled sensitivity for the BinaxNOW kit has been calculated as $69.6 \%$ [3], and reported sensitivities for other kits range from $1.5 \%$ to $97 \%[3,13]$. Decreasing sensitivities at parasite densities below 5,000/ $\mu 1$ and particularly below $500 / \mu 1$ are reported for all kits $[4,14]$. 
Two previous studies assessed the performance of the FK70 in a $P$. vivax-endemic setting. In a prospective clinical study, Lee and co-workers demonstrated sensitivity and specificity of $96.4 \%$ and $98.9 \%$ respectively [15]. However, they did not include data on parasite densities and the control group only consisted of malaria-negative samples. Likewise, Kim and co-workers found in a prospective study an overall sensitivity of $93.4 \%$ with lower sensitivities at low parasite densities, albeit somewhat higher as compared to those found in the present study. Among asymptomatic patients and international travellers with $P$. falciparum infections, they found no false-positive reactions. Compared side-by-side, their results for the FK70 were in line with those obtained with the three-band OptiMAL test (Diamed, Cressier, Switzerland), which detects $P$. vivax through a pan-Plasmodium-pLDH antibody [16]. In contrast to both studies, the FK70 was challenged with a large number of non-vivax species, reflecting more appropriately a travel clinic setting. Unlike the study of Kim and co-workers, this study demonstrated cross-reactions with the Pv-pLDH line in four P. falciparum samples: these samples were obtained in travellers returning from Africa, and co-infection with $P$. vivax was not demonstrated by PCR-analysis. As the presently used PCR analysis had been validated with large numbers of the four main Plasmodium species that mixed infection with $P$. vivax is extremely unlikely and an immunological cross-reaction has to be concluded. Of note is that the three samples in which this cross-reaction was found reproducible had parasite densities exceeding 100,000/ $\mu 1$.

The Pv-pLDH line intensities in the present study were reproducible and reliable but most readings were scored as faint or weak. As noted above, this might be caused by a decrement of antigen activity during storage [3], but this phenomenon was also demonstrated in recently stored samples is also noted in prospectively assessed samples at the ITM reference laboratory. Further studies are to be done in order to confirm and document this observation. Faint test lines are a frequently described problem, which may be caused by the fact that the test line is much narrower than the control line $[17,18]$. The phenomenon is important especially in field conditions: interpretation of faint positive lines as negative test results was found the most common mistake made by community health care workers even when provided with adapted job aids $[19,20]$ and problems can be expected when reading RDTs during evening and night shifts, particularly for readers with poor visual capacities [21].

In our hands, the FK70 proved to be an easy-to-use test. The package insert was scored as clear and informative. The additional job aids is based on the firstly developed WHO generic version of the job aids [22] with easily visible and readable instructions.

Which is the place of a RDT targeting uniquely $P$. vivax? Malaria caused by $P$. vivax is no longer considered as a mild infection, and compiled evidence has demonstrated 
complicated infections in endemic countries as well as in returned travellers [23,24]. Plasmodium vivax is further notorious because of its high transmissibility, which makes it more difficult to eradicate as compared to P. falciparum [25]. It has a tendency to relapse (for which primaquine therapy is needed), and chloroquine resistant strains are reported from various parts in the world [26-28]. As to its epidemiology and geography, it should be noted that $P$. vivax unexpectedly has been recovered from travellers returning from Central-Africa, where it is however rarely found in indigenous populations, due to their lack of the Duffy antigen [29]. In the non-endemic setting, the microscopic differentiation between $P$. vivax and $P$. ovale is notoriously difficult [4], with 14 tot $42 \%$ laboratories participating to external quality assessments confusing the one species with the other [30]. The rare and even decreasing trends of $P$. vivax and $P$. ovale infections in returned travellers contribute to the difficulty of correct species identification [31]. In view of these difficulties, kits such as the FK70 may be a useful aid for the instant differential diagnosis of Plasmodium species. The affordable price, long shelf life and favourable storage conditions of the kit allow purchase for incidental use. At present, the FK70 is used in our laboratory as part of the work-up of samples microscopically suspected for $P$. vivax and/or $P$. ovale. In areas where $P$. vivax is endemic and occurs as a single infection, a $P$. vivax specific RDT can be of value because it is - at an better accuracy - cheaper than a three-band RDT [32,33]. In areas where $P$. vivax and $P$. falciparum are co-circulating, it can distinguish those infections that require treatment with primaquine [3]. However, in view of the potential cross-reaction of $P$. falciparum, the FK70 should not be used the initial or unique screening test in this situation as an occasional concurrent or single $P$. falciparum infection risks to be overlooked.

\section{Conclusions}

In conclusion, the FK70 proved to be easy-to-use and accurate and reliable for the diagnosis of $P$. vivax among a panel of samples of symptomatic international travellers. It is a useful adjunct to microscopy in non-endemic settings. Lower sensitivities at parasite densities below 500/ $\mu$ l and occasional cross-reactions of $P$. falciparum should be taken into account and further prospective investigations should be done to explore the Pv-pLDH line intensities, especially when considering the use of the FK70 in a field setting. 


\section{Abbreviations}

CI: confidence intervals; EDTA: ethylene diamine Tetra-acetic Acid; FK70: SD FK70 Malaria Antigen Pv test; HRP-2: histidine-rich protein-2; ITM: Institute of Tropical Medicine; PCR: polymerase chain reaction; pLDH: parasite lactate dehydrogenase; Pv-pLDH: Plasmodium vivax-specific parasite lactate dehydrogenase; RDT: rapid diagnostic test; WHO: World Health Organization.

\section{Acknowledgements}

We thank the laboratory staff of the Central Laboratory of Clinical Biology for technical support.

\section{References}

1. Hanscheid T: Current strategies to avoid misdiagnosis of malaria. Clin Microbiol Infect 2003, 9: 497-504.

2. Moody A: Rapid diagnostic tests for malaria parasites. Clin Microbiol Rev 2002, 15: 66-78.

3. Murray CK, Gasser RA, Jr., Magill AJ, Miller RS: Update on Rapid Diagnostic Testing for Malaria. Clin Microbiol Rev 2008, 21: 97-110.

4. Wongsrichanalai C, Barcus MJ, Muth S, Sutamihardja A, Wernsdorfer WH: A review of malaria diagnostic tools: microscopy and rapid diagnostic test (RDT). Am J Trop Med Hyg 2007, 77: 119-127.

5. Rougemont M, Van Saanen M, Sahli R, Hinrikson HP, Bille J, Jaton K: Detection of four Plasmodium species in blood from humans by $18 \mathrm{~S}$ rRNA gene subunit-based and speciesspecific real-time PCR assays. J Clin Microbiol 2004, 42: 5636-5643.

6. Bell DR, Wilson DW, Martin LB: False-positive results of a Plasmodium falciparum histidinerich protein 2-detecting malaria rapid diagnostic test due to high sensitivity in a community with fluctuating low parasite density. Am J Trop Med Hyg 2005, 73: 199-203.

7. Iqbal J, Sher A, Rab A: Plasmodium falciparum histidine-rich protein 2-based immunocapture diagnostic assay for malaria: cross-reactivity with rheumatoid factors. $J$ Clin Microbiol 2000, 38: 1184-1186.

8. Bell D, Peeling RW: Evaluation of rapid diagnostic tests: malaria. Nat Rev Microbiol 2006, 4: S34-S38.

9. Seidahmed OM, Mohamedein MM, Elsir AA, Ali FT, Malik eF, Ahmed ES: End-user errors in applying two malaria rapid diagnostic tests in a remote area of Sudan. Trop Med Int Health 2008, 13: 406-409.

10. Marx A, Pewsner D, Egger M, Nuesch R, Bucher HC, Genton B, Hatz C, Juni P : Metaanalysis: accuracy of rapid tests for malaria in travelers returning from endemic areas. Ann Intern Med 2005, 142: 836-846.

11. Wongsrichanalai C, Gasser RA, Jr.: Current status of malaria rapid diagnostic devices: an update. Trends Parasitol 2002, 18: 107-108.

12. World Health Organization. List of known commercially-available antigen-detecting malaria RDTs: information for national public health services and UN Agencies wishing to procure RDTs. 2009.

[http://www.wpro.who.int/NR/rdonlyres/9C624A45-3554-4695-8C11233DB743FC7A/0/MD table23 ISO131485criteriarev041207.pdf]

13. Coleman RE, Maneechai N, Rachapaew N, Kumpitak C, Soyseng V, Miller RS Thimasarn, K Sattabongkot, J: Field evaluation of the ICT Malaria Pf/Pv immunochromatographic test for the detection of asymptomatic malaria in a Plasmodium falciparum/vivax endemic area in Thailand. Am J Trop Med Hyg 2002, 66: 379-383.

14. Forney JR, Wongsrichanalai C, Magill AJ, Craig LG, Sirichaisinthop J, Bautista CT, Miller RS, Ockenhouse CF, Kester KE, Aronson NE, Andersen EM, Quino-Ascurra HA, Vidal C, Moran KA, Murray CK, DeWitt CC, Heppner DG, Kain KC, Ballou WR, Gasser RA Jr.: Devices for Rapid Diagnosis of Malaria: Evaluation of Prototype Assays That Detect Plasmodium 
falciparum Histidine-Rich Protein 2 and a Plasmodium vivax-Specific Antigen. J Clin Microbiol 2003, 41: 2358-2366.

15. Lee SW, Jeon K, Jeon BR, Park I: Rapid diagnosis of vivax malaria by the SD Bioline Malaria Antigen test when thrombocytopenia is present. J Clin Microbiol 2008, 46: 939-942.

16. Kim SH, Nam MH, Roh KH, Park HC, Nam DH, Park GH, Han ET, Klein TA, Lim CS: Evaluation of a rapid diagnostic test specific for Plasmodium vivax. Trop Med Int Health 2008, 13: 1495-1500.

17. Craig MH, Bredenkamp BL, Williams CH, Rossouw EJ, Kelly VJ, Kleinschmidt I, Martineau A, Henry GF: Field and laboratory comparative evaluation of ten rapid malaria diagnostic tests. Trans R Soc Trop Med Hyg 2002, 96: 258-265.

18. McMorrow ML, Masanja MI, Abdulla SM, Kahigwa E, Kachur SP: Challenges in routine implementation and quality control of rapid diagnostic tests for malaria--Rufiji District, Tanzania. Am J Trop Med Hyg 2008, 79: 385-390.

19. Mayxay M, Pukrittayakamee S, Newton PN, White NJ: Mixed-species malaria infections in humans. Trends Parasitol 2004, 20: 233-240.

20. Rennie W, Phetsouvanh R, Lupisan S, Vanisaveth V, Hongvanthong B, Phompida S, Alday P, Fulache M, Lumagui R, Jorgensen P, Bell D, Harvey S: Minimising human error in malaria rapid diagnosis: clarity of written instructions and health worker performance. Trans $R$ Soc Trop Med Hyg 2007, 101: 9-18.

21. Harvey SA, Jennings L, Chinyama M, Masaninga F, Mulholland K, Bell DR: Improving community health worker use of malaria rapid diagnostic tests in Zambia: package instructions, job aid and job aid-plus-training. Malar J 2008, 7: 160.

22. World Health Organization. Generic instruction for malaria rapid test cassette. P.falciparum only. 2009. [http://www.wpro.who.int/NR/rdonlyres/B531CCC0-25A5-4796-A5D0792666032136/0/Generic Cassette 050905 version2.pdf]

23. Habib AG, Singh KS: Respiratory distress in nonimmune adults with imported malaria. Infection 2004, 32: 356-359.

24. Rogerson SJ, Carter R: Severe vivax malaria: newly recognised or rediscovered. PLoS Med 2008, 5: e136.

25. Phimpraphi W, Paul RE, Yimsamran S, Puangsa-art S, Thanyavanich N, Maneeboonyang W, Prommongkol S, Sornklom S, Chaimungkun W, Chavez IF, Blanc H, Looareesuwan S, Sakuntabhai A, Singhasivanon P : Longitudinal study of Plasmodium falciparum and Plasmodium vivax in a Karen population in Thailand. Malar J 2008, 7: 99.

26. Barnadas C, Ratsimbasoa A, Tichit M, Bouchier C, Jahevitra M, Picot S, Menard D : Plasmodium vivax resistance to chloroquine in Madagascar: clinical efficacy and polymorphisms in pvmdr1 and pvcrt-o genes. Antimicrob Agents Chemother 2008, 52: 42334240 .

27. Sattabongkot J, Tsuboi T, Zollner GE, Sirichaisinthop J, Cui L: Plasmodium vivax transmission: chances for control? Trends Parasitol 2004, 20: 192-198.

28. Teka H, Petros B, Yamuah L, Tesfaye G, Elhassan I, Muchohi S, Kokwaro G, Aseffa A, Engers H : Chloroquine-resistant Plasmodium vivax malaria in Debre Zeit, Ethiopia. Malar J 2008, 7: 220 .

29. Culleton RL, Mita T, Ndounga M, Unger H, Cravo PV, Paganotti GM, Takahashi N, Kaneko A, Eto H, Tinto H, Karema C, D'Alessandro U, Do Rosario V, Kobayakawa T, Ntoumi F, Carter R, Tanabe K: Failure to detect Plasmodium vivax in West and Central Africa by PCR species typing. Malar J 2008, 7: 174.

30. Kettelhut MM, Chiodini PL, Edwards H, Moody A: External quality assessment schemes raise standards: evidence from the UKNEQAS parasitology subschemes. J Clin Pathol 2003, 56: 927-932.

31. Smith AD, Bradley DJ, Smith V, Blaze M, Behrens RH, Chiodini PL, Whitty CJ : Imported malaria and high risk groups: observational study using UK surveillance data 1987-2006. BMJ 2008, 337: a120.

32. Lee SW, Jeon K, Jeon BR, Park I: Rapid diagnosis of vivax malaria by the SD Bioline Malaria Antigen test when thrombocytopenia is present. J Clin Microbiol 2008, 46: 939-942.

33. World Health Organization. Interim notes on selection of type of malaria rapid diagnostic test in relation to the occurrence of different parasite species. 2009. [http://www.wpro.who.int/NR/rdonlyres/CF152D7C-25BA-49E7-86D2-

DBEE4E5B5974/0/INTERIMNOTESONMALARIARDTS RBM final2 rev3.pdf] 


\section{Chapter IIb}

\section{Laboratory evaluation of malaria RDTs}

targeting $P$. vivax

IIb: Test characteristics of the SD FK80 Plasmodium falciparum/Plasmodium vivax malaria rapid diagnostic test in a non-endemic setting.

Gillet P, van Dijk DP, Bottieau E, Cnops L, Van Esbroeck M, Jacobs J.

Malaria Journal 2009; 8:262.

http://www.malariajournal.com/content/8/1/262 
Test characteristics of the SD FK80 Plasmodium falciparum/Plasmodium vivax malaria rapid diagnostic test in a non-endemic setting

\begin{tabular}{|c|}
\hline bs \\
\hline $\begin{array}{l}\text { Background } \\
\text { The SD FK } 80 \text { P.f/P.v Malaria Antigen Rapid Test (Standard Diagnostics, Korea) } \\
\text { (FK80) is a three-band malaria rapid diagnostic test detecting Plasmodium } \\
\text { falciparum Histidine-Rich Protein-2 (HRP-2) and Plasmodium vivax-specific lactate } \\
\text { dehydrogenase (Pv-pLDH). The present study assessed its performance in a non- } \\
\text { endemic setting. }\end{array}$ \\
\hline $\begin{array}{l}\text { Methods } \\
\text { Stored blood samples }(\mathrm{n}=416) \text { from international travellers suspected of malaria } \\
\text { were used, with microscopy corrected by PCR as the reference method. Samples } \\
\text { infected by Plasmodium falciparum }(\mathrm{n}=178) \text {, Plasmodium vivax }(\mathrm{n}=99) \text {, } \\
\text { Plasmodium ovale }(\mathrm{n}=75) \text { and Plasmodium malariae }(\mathrm{n}=24) \text { were included, as } \\
\text { well as } 40 \text { malaria negative samples. }\end{array}$ \\
\hline $\begin{array}{l}\text { Results } \\
\text { Overall sensitivities for the diagnosis of } P \text {. falciparum and } P \text {. vivax were } 91.6 \% \\
(95 \% \text { confidence interval }(\mathrm{CI}): 86.2 \%-95.0 \%) \text { and } 75.8 \%(65.9 \%-83.6 \%) \text {. For } P \text {. } \\
\text { falciparum, sensitivity at parasite densities } \geq 100 / \mu \text { was } 94.6 \%(88.8 \%-97.6 \%) \text {; } \\
\text { for } P \text {. vivax, sensitivity at parasite densities } \geq 500 / \mu \text { l was } 86.8 \%(75.4 \%-93.4 \%) \text {. } \\
\text { Four } P \text {. falciparum samples showed a Pv-pLDH line, three of them had parasite } \\
\text { densities exceeding } 50.000 / \mu 1 \text {. Two } P \text {. vivax samples, one } P \text {. ovale and one } P \text {. } \\
\text { malariae sample showed a HRP-2 line. For the HRP-2 and Pv-pLDH lines, } \\
\text { respectively } 81.4 \%(136 / 167) \text { and } 55.8 \% \text { (43/77) of the true positive results were } \\
\text { read as medium or strong line intensities. The FK80 showed good reproducibility } \\
\text { and reliability for test results and line intensities (kappa values for both exceeding } \\
0.80) \text {. }\end{array}$ \\
\hline $\begin{array}{l}\text { Conclusion } \\
\text { The FK } 80 \text { test performed satisfactorily in diagnosing } P \text {. falciparum and } P \text {. vivax } \\
\text { infections in a non-endemic setting. }\end{array}$ \\
\hline
\end{tabular}




\section{Background}

Malaria rapid diagnostic tests (RDTs) are immunochromatographic tests that detect circulating antigens of Plasmodium species. They are an adjunct to the microscopic diagnosis of malaria, both in the endemic and in the non-endemic settings [1-5]. RDTs are built on a nitrocellulose platform and are available in dipstick or cassette format. Signals are visible as coloured lines, comprising a control line (which indicates that the test has been performed well) and one or two test lines. The socalled two band tests generate a test line that targets $P$. falciparum by detecting either histidine-rich protein 2 (HRP-2) or P. falciparum-specific parasite lactate dehydrogenase (Pf-pLDH). The three band tests include a second target that is common to the four Plasmodium species, such as aldolase or pan-specific parasite lactate dehydrogenase (pan-pLDH). Apart from these common formats, there are also two-band tests detecting Plasmodium vivax-specific $\mathrm{pLDH}$ (Pv-pLDH) [6]. The SD FK80 P.f/P.v Malaria Antigen Rapid Test (Standard Diagnostics, Hagal-Dong, Republic of Korea), further referred to as FK80, is a three-band RDT that targets HRP-2 and Pv-pLDH. In areas where both $P$. falciparum and $P$. vivax are prevalent, this combination has the advantage to distinguish unequivocally between the two species, whereas a conventional three band $P$. falciparum/Pan species RDT may not. For instance, in case of a single $P$. falciparum infection, the FK80 P.f/P.v Malaria Antigen Rapid Test will only show a unique HRP-2 line; in this case the conventional three band $P$. falciparum/Pan species RDT may show (depending on the parasite density) both the HRP-2 (or Pf-pLDH line) and the pan-species line, a result which is also compatible with a $P$. falciparum/P. vivax mixed infection. In addition, it can be assumed that the affinity of a dedicated antibody targeting the enzyme of only a single species (Pv-pLDH in the case of $P$. vivax and the current FK80 RDT) is higher than the affinity of an antibody targeting the different enzymes of the four Plasmodium species (pan-pLDH). The aim of the study was to evaluate the performance of the FK80 in a non-endemic reference setting.

\section{Methods}

\section{Study design}

In this retrospective study, the FK80 was evaluated against a collection of stored samples obtained from international travellers, collected from January 1997 to December 2008. Tests were carried out at the malaria reference laboratory of the Institute of Tropical Medicine (ITM) Antwerp, Belgium. This study fulfils the standards for the reporting of diagnostic accuracy studies (STARD) checklist.

\section{Patients and samples}

Samples were selected from a collection of EDTA-blood samples stored at $-70^{\circ} \mathrm{C}$ and obtained from patients presenting at the outpatient clinic of ITM for clinical suspicion of malaria. The patients were international travellers and, to a lesser 
extent, immigrants returning from visits to their native countries. In addition, samples sent by Belgian laboratories to ITM in the scope of the national reference function were included. The samples collected at ITM, were aliquoted and frozen at $-70^{\circ} \mathrm{C}$ the day of collection. Between collection and storage at $-70^{\circ} \mathrm{C}$, the samples remained at room temperature for a maximum of 10 hours, at ambient temperatures below $25^{\circ} \mathrm{C}$. The 99 samples sent by Belgian laboratories to ITM for second opinion and confirmation were sent by mail and had been exposed to ambient temperature for the period of shipment, which was generally less than 24 hours and ranged to a maximum of 48 hours. The delays of shipment and processing before storage at $70^{\circ} \mathrm{C}$ had been validated before and were compliant with routine laboratory procedures. A representative number of Plasmodium-positive samples $(n=376)$ were selected, including the four malaria species with varying parasite densities and representative geographic distribution. Mixed infections were not included. In addition, samples without malaria parasites (as confirmed by microscopy and PCR) from symptomatic travellers were included (negative samples, $\mathrm{n}=40$ ).

\section{Reference method}

Microscopy corrected by polymerase chain reaction (PCR) was used as the reference method. Standard microscopy was performed on thick blood films of all samples to diagnose malaria and to assess parasite density, and on thin blood films of positive samples to define the Plasmodium species. Thick blood films were stained with Giemsa ( $\mathrm{pH}=8$ ) for 20 minutes, thin films with May-Grünwald Giemsa. The slides were examined by light microscopy using a $\times 500$ magnification, according to the standard procedure at ITM. Parasite density was assessed by counting the number of asexual parasites against 200 white blood cells in a thick film, and converting the value to parasites/ $\mu 1$ using the actual count or the standard of 8,000 white blood cell/ $\mu 1$ [3]. Malaria diagnosis at Central Laboratory of Clinical Biology is accredited in accordance with the requirements of the ISO 15189:2007 norm. The laboratory technicians have received a detailed training and their performance and agreement are monitored by participation to internal and external quality control assessments. As a standard procedure, all positive slides for malaria and slides showing discordant results between different laboratory tests (microscopy, RDTs or PCR) are confirmed by a blinded second microscopist. Real-time PCR analysis was adapted from Rougemont et al [7] as described previously [8] and performed on all samples used in this study.

\section{Test platform}

The FK80 is lateral flow immunochromatographic RDT in a cassette format. Three lines are present, a control line which indicates whether the test is valid, a HRP-2 line and a Pv-pLDH line. According to the manufacturer's instructions, a single HRP-2 line indicates an infection with $P$. falciparum, a single Pv-pLDH line 
indicates an infection with $P$. vivax and a combination of a HRP-2 line and a PvpLDH line indicates a mixed infection with $P$. falciparum and $P$. vivax. The other Plasmodium species cannot be detected with this test. For the evaluation, test kits of lot number RDT 8002 (expiry date November 2010) were used.

\section{Test procedure}

Tests were according to the instructions of the manufacturer except that samples $(5 \mu 1)$ where loaded with a transfer pipette (Finnpipette, Helsinki, Finland) instead of the plastic loop supplied by the manufacturer and that a scoring system was used to assess the intensity of the test lines. In case the control line did not appear, the result was interpreted as invalid and the test was repeated. In order to score test line intensities, the scoring system of Bell and co-workers was adapted [9] and five categories were defined: none (no line visible), faint (barely visible line), weak (paler than the control line), medium (equal to the control line) or strong (stronger than the control line) [8]. To assure timely readings, tests were carried out in timecontrolled batches of ten samples. Readings were carried out at daylight assisted by a standard electricity bulb, between 20 and not beyond 30 minutes after application of the sample and buffer. Readings were performed by three subsequent observers, of whom the one who performed the test procedure was the first. Observers were blinded to the results of microscopy, PCR and to each others' readings. The results of the readings considered were based on consensus agreement, which means that a positive result was defined as a result read positive by at least two out of three different observers. When there was no consensus, results of the first observer were considered. Inter-reader reliabilities were assessed for the test results expressed as positive and negative readings as well as for the intensity readings. To assess test reproducibility, a panel of 19 samples (including nine $P$. falciparum samples, eight $P$. vivax samples, one $P$. ovale sample and one $P$. malariae sample) was tested on three successive occasions.

\section{Definitions}

Samples infected with $P$. falciparum and $P$. vivax species were considered separately: Tables 1 and 2 list the definitions of true positive and negatives and those of species mismatches. In the case of $P$. falciparum, samples with pure gametocytaemia were included among the positive samples.

\section{Statistical analysis}

Sensitivity and specificity were calculated for both $P$. falciparum and $P$. vivax with 95\% confidence intervals (CI) and differences were tested for significance using the Pearson chi-square test or, when this was not possible, the Fisher exact probability test. A p-value of $<0.05$ was considered as significant. Reliabilities for positive and negative readings and line intensities were calculated as percentage agreements for 
all three readers and kappa values for each pair of readers. Associations between line intensity readings and parasite densities were assessed for strength of association with Cramer's $V$ for categorical variables.

Table 1: Interpretation of test results of the FK80 for the detection of P. falciparum.

\begin{tabular}{|c|c|c|}
\hline \multirow[b]{2}{*}{ Test Line(s) visible } & \multicolumn{2}{|c|}{ Species identification by microscopy corrected by PCR } \\
\hline & P. falciparum & $\begin{array}{c}P . \text { vivax, } P . \text { ovale, } P . \text { malariae } \\
\text { or no parasites detected }\end{array}$ \\
\hline Only HRP-2 & True positive & $\begin{array}{c}\text { False positive } \\
\text { or } \\
\text { species-mismatch* }\end{array}$ \\
\hline $\begin{array}{l}\text { None or } \\
\text { only Pv-pLDH or } \\
\text { HRP-2 + Pv-pLDH }\end{array}$ & $\begin{array}{c}\text { False negative } \\
\text { or } \\
\text { species-mismatch** }\end{array}$ & True negative \\
\hline
\end{tabular}

* Non-falciparum species diagnosed as P. falciparum

** $P$. falciparum diagnosed as $P$. vivax or as $P$. falciparum/ $P$. vivax mixed infection

Table 2: Interpretation of test results of the FK80 for the detection of $P$. vivax.

\begin{tabular}{|c|c|c|}
\hline \multirow[b]{2}{*}{ Test Line(s) visible } & \multicolumn{2}{|c|}{ Species identification by microscopy corrected by PCR } \\
\hline & $P$. vivax & $\begin{array}{l}P . \text { falciparum, } P . \text { ovale, } P . \text { malariae } \\
\text { or no parasites detected }\end{array}$ \\
\hline Only Pv-pLDH & True positive & $\begin{array}{c}\text { False positive } \\
\text { or } \\
\text { species-mismatch* }\end{array}$ \\
\hline $\begin{array}{l}\text { None or } \\
\text { only HRP-2 or } \\
\text { both HRP-2 and Pv- } \\
\text { pLDH }\end{array}$ & $\begin{array}{c}\text { False negative } \\
\text { or } \\
\text { species-mismatch** }\end{array}$ & True negative \\
\hline
\end{tabular}

\section{Ease of use}

Three experienced laboratory technicians scored the ease of use of the FK80 test and the clarity of manufacturer's instructions with a standardized list.

\section{Ethical review}

The study was reviewed and approved by the Institutional Review Board of ITM and by the Ethical Committee of Antwerp University, Belgium. 


\section{Results}

\section{Sample collection}

A total of 416 samples were selected, of which 99 samples were sent by Belgian laboratories to ITM for second opinion and confirmation. The samples were collected from January 1997 to December 2008.

According to microscopy and after correction with PCR analysis, 178 of these samples were positive for P. falciparum, 99 for $P$. vivax, 75 for $P$. ovale and 24 for $P$. malariae. The results of microscopy were corrected in 11 out of the 376 positive samples $(2.9 \%)$ and were uniquely related to $P$. vivax - $P$. ovale mismatches: in the final collection, four out of $99(4.0 \%)$ P. vivax samples and seven out of $74(9.5 \%)$ $P$. ovale samples had been identified as $P$. ovale and $P$. vivax by microscopy. In addition, 40 microscopic and PCR negative samples of symptomatic travellers were included in the panel.

\section{Invalid test results}

One of the 416 samples gave an invalid result at initial testing. After 30 minutes, there was no control line visible. Upon repetition, the test performed well.

Table 3: Test results for the FK80 for all samples $(n=416)$.

\begin{tabular}{|c|c|c|c|c|c|}
\hline \multirow[b]{2}{*}{ Sample } & \multicolumn{2}{|c|}{ HRP-2 line positive } & \multicolumn{2}{|c|}{ HRP-2 line negative } & \multirow[b]{2}{*}{ Total } \\
\hline & $\begin{array}{c}\text { Pv-pLDH } \\
\text { line } \\
\text { positive }\end{array}$ & $\begin{array}{c}\text { Pv-pLDH } \\
\text { line } \\
\text { negative }\end{array}$ & $\begin{array}{c}\text { Pv-pLDH } \\
\text { line } \\
\text { positive }\end{array}$ & $\begin{array}{l}\text { Pv-pLDH } \\
\text { line } \\
\text { negative }\end{array}$ & \\
\hline P. falciparum & $4 *$ & 163 & & 11 & 178 \\
\hline P. vivax & $2^{*}$ & & 75 & 22 & 99 \\
\hline P. ovale & & $1 *$ & & 74 & 75 \\
\hline P. malariae & & $1 *$ & & 23 & 24 \\
\hline Negative & & & & 40 & 40 \\
\hline Total & 6 & 165 & 75 & 170 & 416 \\
\hline
\end{tabular}

\section{Sensitivity and specificity}

RDTs were performed between January and February 2009. Table 3 lists the test results of all samples. Test characteristics matched with species identification and parasite density for the detection of $P$. falciparum and $P$. vivax are listed in tables 4 and 5 respectively. For the detection of $P$. falciparum the sensitivity was $91.6 \%$ and the specificity was $99.2 \%$ for all samples combined. Sensitivity was higher at higher parasite densities, but the differences did not reach statistical significance. For the 
detection of $P$. vivax the sensitivity was $75.8 \%$ and the specificity was $100 \%$ for all samples combined. Sensitivity at parasite densities $\leq 500 / \mu 1$ was significantly lower compared to sensitivity at parasite densities $>500 / \mu 1(51.6 \%$ and $86.8 \%$ respectively $(\mathrm{p}<0.001)$ ). Eight out of the 416 samples resulted in a species mismatch, with $4 / 178$ (2.2\%) P. falciparum samples showing a Pv-pLDH line in addition to the HRP-2 line: three of them had parasite densities higher than $1 \%(69,953 ; 87,149$ and $1,000,000 / \mu 1$ respectively) and all four patients were upon return from Africa (travel destination were Nigeria $(n=3)$ and Cameroon). Another four out of $199(2.0 \%)$ non-falciparum samples showed a HRP-2 line (of which two $P$. vivax samples in addition to the Pv-pLDH line) (Table 3). None of the $P$. ovale samples showed positive readings with the $\mathrm{Pv}-\mathrm{pLDH}$ line.

Table 4: Test characteristics of the FK80 for the detection of $P$. falciparum related to parasite densities $(\mathrm{n}=416)$.

\begin{tabular}{|c|c|c|c|c|}
\hline Species & Numbers & $\begin{array}{c}\text { Correctly } \\
\text { identified } \\
\text { by } \\
\text { SDFK80* }\end{array}$ & $\begin{array}{c}\text { Sensitivity } \\
\text { in \% } \\
(95 \% \text { CI })\end{array}$ & $\begin{array}{c}\text { Specificity } \\
\text { in \% } \\
(95 \% \mathrm{CI})\end{array}$ \\
\hline All P. falciparum samples & 178 & 163 & $\begin{array}{c}91.6 \\
(86.2-95.0)\end{array}$ & \\
\hline Pure gametocytaemia & 22 & 17 & $\begin{array}{c}77.3 \\
(54.2-91.3)\end{array}$ & \\
\hline Parasite density $0-100 / \mu 1$ & 26 & 23 & $\begin{array}{c}88.5 \\
(68.7-97.0)\end{array}$ & \\
\hline $\begin{array}{l}\text { Parasite density } 101 \text { - } \\
1,000 / \mu 1\end{array}$ & 24 & 22 & $\begin{array}{c}91.7 \\
(71.5-98.5)\end{array}$ & \\
\hline Parasite density $>1,000 / \mu 1$ & 106 & 101 & $\begin{array}{c}95.3 \\
(88.8-98.3)\end{array}$ & \\
\hline Parasite density $>100 / \mu 1$ & 130 & 123 & $\begin{array}{c}94.6 \\
(88.8-97.6)\end{array}$ & \\
\hline $\begin{array}{l}\text { Non-falciparum or } \\
\text { no parasites seen }\end{array}$ & 238 & $2 * *$ & & $\begin{array}{c}99.2 \\
(96.7-99.9)\end{array}$ \\
\hline
\end{tabular}

* Unique HRP-2 line visible

** One $P$. ovale and one $P$. malariae sample showed a unique HRP-2 line 


\section{Line intensities}

For the HRP-2 and Pv-pLDH lines, respectively 81.4\% (136/167) and 55.8\% (43/77) of the true positive results were read as medium or strong line intensities. For HRP2, faint lines occurred only in three samples: one $P$. falciparum sample with pure gametocytaemia and two non-falciparum samples. For Pv-pLDH, faint lines occurred only in eight samples: six $P$. vivax samples (four of them with a parasitemia lower than 500 parasites $/ \mu \mathrm{l}$ ) and two $P$. falciparum samples. Line intensity readings for both HRP-2 and Pv-pLDH lines were related to parasite densities (HRP-2: $V=0.366, \mathrm{p}<0.001$; Pv-pLDH: $V=0.448, \mathrm{p}<0.001$ ), but there was a considerable overlap.

Table 5: Test characteristics of the FK80 for the detection of $P$. vivax according to parasite densities $(\mathrm{n}=$ 416).

\begin{tabular}{|c|c|c|c|c|}
\hline Species & Numbers & $\begin{array}{c}\text { Correctly } \\
\text { identified by } \\
\text { SDFK80* }\end{array}$ & $\begin{array}{l}\text { Sensitivity } \\
\text { in \% } \\
(95 \% \mathrm{CI}) \\
\end{array}$ & $\begin{array}{c}\text { Specificity } \\
\text { in \% } \\
(95 \% \mathrm{CI}) \\
\end{array}$ \\
\hline All $P$. vivax samples & 99 & 75 & $\begin{array}{c}75.8 \\
(65.9-83.6)\end{array}$ & \\
\hline Parasite density $0-500 / \mu 1$ & 31 & 16 & $\begin{array}{c}51.6 \\
(33.4-62.4)\end{array}$ & \\
\hline Parasite density $501-1,000 / \mu 1$ & 9 & 9 & $\begin{array}{c}100 \\
(62.9-100)\end{array}$ & \\
\hline Parasite density $>1,000 / \mu 1$ & 59 & 50 & $\begin{array}{c}84.7 \\
(72.5-92.4)\end{array}$ & \\
\hline Parasite density $>500 / \mu 1$ & 68 & 59 & $\begin{array}{c}86.8 \\
(75.4-93.4)\end{array}$ & \\
\hline Non-vivax or no parasites seen & 317 & 0 & & $\begin{array}{c}100 \\
(98.5-100)\end{array}$ \\
\hline
\end{tabular}

*Unique Pv-pLDH line visible

\section{Inter-reader reliability}

For the HRP-2 line, the inter-reader reliability for positive and negative test results was excellent, with $98.0 \%$ agreement between the three readers and kappa values between the different pairs of readers of $0.99,0.97$ and 0.99 respectively. For the PvpLDH line, the inter-reader reliability for positive and negative test results was also 
excellent, with $98.3 \%$ overall agreement and kappa values of $0.97,0.97$ and 0.97 for the different pairs of readers.

In terms of line intensity readings, the overall agreement for the HRP-2 line was $90.2 \%$, with kappa values of $0.88,0.91$ and 0.88 for the three pairs of readers. For the $\mathrm{Pv}$-pLDH intensity readings, there was a $91.6 \%$ overall agreement with kappa values of $0.80,0.91$ and 0.80 between the three pairs of readers.

\section{Reproducibility}

With regard to line intensity readings, HRP-2 lines were consistently read upon three times repetition for 14 out of 19 samples. All non-consistent samples had identical test results upon two times repetition and for a single sample there was a difference of two categories in line intensity (weak versus negative). Pv-pLDH intensity readings were consistent upon three times repetition for 17 out of 19 samples. The two remaining samples had consistent results upon two times repetition, and one of them had a single negative result versus two strong line intensity results. In terms of test results, this meant that two $P$. falciparum samples and a single $P$. vivax sample gave false-negative results in one out of three test repetitions.

\section{Ease of use}

The FK80 was scored as easy to use and the instructions were scored as clear and simple to perform by all three technicians. The test was evaluated as practical and the clearance of the test strip was good. However, the clearance of the test strip was incomplete after the 15 minutes minimal incubation time recommended by the manufacturer. Good clearance was observed only after 20 minutes of waiting time.

\section{Discussion}

The present study evaluated the performance of the FK80 against a panel of whole blood samples from international travellers suspected of malaria. The retrospective design of the study has its limitations. For instance, whole blood specimens stored at $-70^{\circ} \mathrm{C}$ were used, and the stability of the target antigens under freezing conditions has been questioned [10]. However, no obvious differences in test performance were presently found for samples stored for several (>5) years compared to those stored for a shorter periods (results not shown). In addition, a prospective evaluation of fresh and stored samples revealed similar results in case of the HRP-2 antigen detection [11]. Another limitation is the fact that a calibrated pipette was used for the transfer of the blood; with an expected better accuracy as compared to the kit's application loop. In addition, the diagnosis and evaluation were carried out in a reference setting, which makes extrapolation of the present results to field settings difficult $[4,10]$. Likewise, the ease of use was checked by an expert team and not by untrained end users in remote areas, and it is known that expert technicians tend to score tests kits more favorably [12]. 
The performance of the FK80 can best be compared to other tests by considering the HRP-2 and Pv-pLDH lines separately. For the HRP-2 test line in the diagnosis of $P$. falciparum, the sensitivities were in line with those reported in other HRP-2 tests in returned travellers, with sensitivities ranging from $80 \%$ tot $99 \%$, depending on the setting and parasite densities [2,13-19]. However, the FK80 did not meet the 95\% sensitivity at 100 parasites/ $\mu \mathrm{l}$ as recommended by the WHO [20]. Concerning the diagnosis of $P$. vivax, comparison is more difficult. There are three reports on the performance of the Pv-pLDH detection system in the SD FK70 test, a two-band $P$. vivax RDT marketed by the same company. Two field studies reported sensitivities of $96.4 \%$ and $93.4 \%$ respectively, with lower values at low parasite densities $[21,22]$. In addition, the FK70 was evaluated in the present reference setting on stored whole blood samples from travellers [6]: this study demonstrated an overall sensitivity of $88.0 \%$, with sensitivities at parasite densities below and above $500 / \mu 1$ of $64.3 \%$ and $97.2 \%$ respectively. Comparison of the sensitivities of the FK80 PvpLDH detection with those reported for the pan-pLDH target are even more difficult, as the latter range from $1.5 \%$ to $97.0 \%$ [23,24]. Compiled sensitivities for the BinaxNOW kit for the diagnosis of $P$. vivax was reported as $69.6 \%$ [24]. With regard to specificity, there was no reaction of any of the $P$. ovale samples with the Pv-pLDH test line, but two $P$. vivax samples gave positive readings with the HRP-2 line in addition to the $\mathrm{Pv}-\mathrm{pLDH}$ line. Of note is the $\mathrm{Pv}-\mathrm{pLDH}$ positive result in four $P$. falciparum samples: in all cases PCR as well as travel destination argued against $P$. vivax co-infection, and three of them occurred at higher parasite densities. This is in line with earlier observations, in which occasional cross-reaction of $P$. falciparum at high parasite densities was observed in the FK70 [6].

The HRP-2 lines generally were of higher intensities as compared to the Pv-pLDH lines. The higher line intensities of the HRP-2 line as compared to the pan-pLDH and aldolase lines have been described previously [8,25]. For both the test lines, the line intensities of true positive results were higher as compared to the previously evaluated FK60 and FK70 tests [6,8].

The intended area of use of the presently evaluated FK80 RDT is confined to regions with both $P$. falciparum and $P$. vivax infections, such as Afghanistan or Korea. In such situation, the FK80 has an advantage over the traditional three-band combination RDTs that detect HRP-2 and pan-species specific pLDH of aldolase: unlike the FK80, their design does not allow to exclude a mixed infection in the case of $P$. falciparum samples that react with both the HRP-2 and the pan-species lines. The FK80 by virtue of its specific Pv-pLDH is able to distinguish accurately between infections caused by $P$. falciparum, $P$. vivax and both species combined. Accurate diagnosis of $P$. vivax is important in view of the need for specific therapy but may also be interesting because of the study of $P$. vivax malaria, because this infection may be serious [26], requires a specific treatment and is also notorious because of its high transmissibility [27,28]. In other regions where these two species 
co-exist, the presence of $P$. ovale and $P$. malariae can not be excluded and a threeband test with a pan-species pLDH will be a better choice. In the setting of travel medicine, the FK80 may help in distinguishing mixed $P$. falciparum $-P$. vivax infections and in the distinction between $P$. vivax and $P$. ovale. However, as in this setting cost is not a prohibitive factor, a standard three-band RDT followed by, in case of single pan-pLDH positivity, a two-band Pv-pLDH RDT such as the FK70 is an alternative to support the microscopic diagnosis between $P$. ovale and $P$. vivax [6].

In conclusion, the FK80 performed satisfactorily for the diagnosis of $P$. falciparum and $P$. vivax infections in a non-endemic setting, especially at higher parasite densities.

\section{List of abbreviations}

Ag: Antigen; CI: Confidence Interval; DNA: Desoxy-riboNucleic Acid; EDTA: Ethylene Diamine Tetra-acetic Acid; FHML: Faculty of Health Medicine and Life Sciences, Maastricht, The Netherlands; FK60: SD FK60 Malaria Ag $P$. falciparum/Pan test (Standard Diagnostics), a three-band "one-step" malaria rapid diagnostic test for the detection of $P$. falciparum and non-falciparum species, targeting the HRP-2 and pLDH antigens; FK70: SD FK70 Malaria Ag. P. vivax test (Standard Diagnostics), a two-band "one-step" malaria rapid diagnostic test for the detection of $P$. vivax, targeting the Pv-pLDH antigen; FK80: SD FK80 Malaria Ag. $P$. falciparum/P. vivax test (Standard Diagnostics), a three-band "one step" malaria rapid diagnostic test for the detection of $P$. falciparum and $P$. vivax, targeting the HRP-2 and Pv-pLDH antigens; HRP-2: Histidine Rich Protein-2; ITM: Institute of Tropical Medicine, Antwerp, Belgium; RDT(s): Malaria Rapid Diagnostic Test(s); P.: Plasmodium; Pan-pLDH: Pan-specific Parasite Lactate Dehydrogenase; PCR: Polymerase Chain Reaction; Pf-pLDH: Plasmodium falciparum-specific Parasite Lactate Dehydrogenase; pLDH: Parasite Lactate Dehydrogenase; Pv-pLDH: Plasmodium vivax-specific parasite Lactate Dehydrogenase; STARD: Standards for the reporting of diagnostic accuracy studies

\section{Acknowledgements}

We would like to thank the staff of the Central Laboratory of Clinical Biology for technical support. 


\section{References}

1. Hanscheid T: Current strategies to avoid misdiagnosis of malaria. Clin Microbiol Infect 2003, 9: 497-504.

2. Marx A, Pewsner D, Egger M, Nuesch R, Bucher HC, Genton B, Hatz C, Juni P: Meta-analysis: accuracy of rapid tests for malaria in travelers returning from endemic areas. Ann Intern Med 2005, 142: 836-846.

3. Moody A: Rapid diagnostic tests for malaria parasites. Clin Microbiol Rev 2002, 15: 66-78.

4. Murray CK, Gasser RA, Jr., Magill AJ, Miller RS: Update on Rapid Diagnostic Testing for Malaria. Clin Microbiol Rev 2008, 21: 97-110.

5. Wongsrichanalai C, Barcus MJ, Muth S, Sutamihardja A, Wernsdorfer WH: A review of malaria diagnostic tools: microscopy and rapid diagnostic test (RDT). Am J Trop Med Hyg 2007, 77: 119-127.

6. Gillet P, Bosselaers K, Cnops L, Bottieau E, Van Esbroeck M., Jacobs J: Evaluation of the SD FK70 malaria Ag Plasmodium vivax rapid diagnostic test in a non-endemic setting. Malar $J$ 2009, 8: 129.

7. Rougemont M, Van Saanen M, Sahli R, Hinrikson HP, Bille J, Jaton K: Detection of four Plasmodium species in blood from humans by $18 \mathrm{~S}$ rRNA gene subunit-based and speciesspecific real-time PCR assays. J Clin Microbiol 2004, 42: 5636-5643.

8. Van der Palen M., Gillet P, Bottieau E, Cnops L, Van Esbroeck M., Jacobs J: Test characteristics of two rapid antigen detection tests (SD FK50 and SD FK60) for the diagnosis of malaria in returned travellers. Malar $J$ 2009, 8: 90.

9. Bell DR, Wilson DW, Martin LB: False-positive results of a Plasmodium falciparum histidine-rich protein 2-detecting malaria rapid diagnostic test due to high sensitivity in a community with fluctuating low parasite density. Am J Trop Med Hyg 2005, 73: 199-203.

10. Bell D, Peeling RW: Evaluation of rapid diagnostic tests: malaria. Nat Rev Microbiol 2006, 4: S34-S38.

11. Mayxay M, Pukrittayakamee S, Chotivanich K, Looareesuwan S, White NJ: Persistence of Plasmodium falciparum HRP-2 in successfully treated acute falciparum malaria. Trans $R$ Soc Trop Med Hyg 2001, 95: 179-182.

12. Seidahmed OM, Mohamedein MM, Elsir AA, Ali FT, Malik eF, Ahmed ES: End-user errors in applying two malaria rapid diagnostic tests in a remote area of Sudan. Trop Med Int Health 2008, 13: 406-409.

13. De Monbrison F, Gerome P, Chaulet JF, Wallon M, Picot S, Peyron F: Comparative diagnostic performance of two commercial rapid tests for malaria in a non-endemic area. Eur J Clin Microbiol Infect Dis 2004, 23: 784-786.

14. Durand F, Crassous B, Fricker-Hidalgo H, Carpentier F, Brion JP, Grillot R, Pelloux H: Performance of the Now Malaria rapid diagnostic test with returned travellers: a 2-year retrospective study in a French teaching hospital. Clin Microbiol Infect 2005, 11: 903-907.

15. Farcas GA, Zhong KJ, Lovegrove FE, Graham CM, Kain KC: Evaluation of the Binax NOW ICT test versus polymerase chain reaction and microscopy for the detection of malaria in returned travelers. Am J Trop Med Hyg 2003, 69: 589-592.

16. Gatti S, Gramegna M, Bisoffi Z, Raglio A, Gulletta M, Klersy C, Bruno A, Maserati R, Madama S, Scaglia M: A comparison of three diagnostic techniques for malaria: a rapid diagnostic test (NOW Malaria), PCR and microscopy. Ann Trop Med Parasitol 2007, 101: 195-204.

17. Grobusch MP, Hanscheid T, Gobels K, Slevogt H, Zoller T, Rogler G, Teichmann D: Comparison of three antigen detection tests for diagnosis and follow-up of falciparum malaria in travellers returning to Berlin, Germany. Parasitol Res 2003, 89: 354-357.

18. Jelinek T, Grobusch MP, Schwenke S, Steidl S, von Sonnenburg F, Nothdurft HD, Klein E, Loscher T: Sensitivity and Specificity of Dipstick Tests for Rapid Diagnosis of Malaria in Nonimmune Travelers. J Clin Microbiol 1999, 37: 721-723.

19. Van Den Ende J, Vervoort T, Van Gompel A, Lynen L: Evaluation of two tests based on the detection of histidine rich protein 2 for the diagnosis of imported Plasmodium falciparum malaria. Trans R Soc Trop Med Hyg 1998, 92: 285-288.

20. World Health Organization. Regional Office for the Western Pacific 2003. Malaria Rapid Diagnosis: Making it Work. Meeting report 20-23 January 2003. Manila, the Philippines. 2003. [http://www.searo.who.int/LinkFiles/Malaria_MalariaRDT.pdf] 
21. Kim SH, Nam MH, Roh KH, Park HC, Nam DH, Park GH, Han ET, Klein TA, Lim CS: Evaluation of a rapid diagnostic test specific for Plasmodium vivax. Trop Med Int Health 2008, 13: 1495-1500.

22. Lee SW, Jeon K, Jeon BR, Park I: Rapid diagnosis of vivax malaria by the SD Bioline Malaria Antigen test when thrombocytopenia is present. J Clin Microbiol 2008, 46: 939-942.

23. Coleman RE, Maneechai N, Rachapaew N, Kumpitak C, Soyseng V, Miller RS, Thimasarn K, Sattabongkot J: Field evaluation of the ICT Malaria Pf/Pv immunochromatographic test for the detection of asymptomatic malaria in a Plasmodium falciparum/vivax endemic area in Thailand. Am J Trop Med Hyg 2002, 66: 379-383.

24. Murray CK, Bell D, Gasser RA, Wongsrichanalai C: Rapid diagnostic testing for malaria. Trop Med Int Health 2003, 8: 876-883.

25. Richter J, Gobels K, Muller-Stover I, Hoppenheit B, Haussinger D: Co-reactivity of plasmodial histidine-rich protein 2 and aldolase on a combined immuno-chromographic-malaria dipstick (ICT) as a potential semi-quantitative marker of high Plasmodium falciparum parasitaemia. Parasitol Res 2004, 94: 384-385.

26. Barnadas C, Ratsimbasoa A, Tichit M, Bouchier C, Jahevitra M, Picot S, Menard D: Plasmodium vivax resistance to chloroquine in Madagascar: clinical efficacy and polymorphisms in pvmdr1 and pvcrt-o genes. Antimicrob Agents Chemother 2008, 52: 42334240 .

27. Phimpraphi W, Paul RE, Yimsamran S, Puangsa-art S, Thanyavanich N, Maneeboonyang W, Prommongkol S, Sornklom S, Chaimungkun W, Chavez IF, Blanc H, Looareesuwan S, Sakuntabhai A, Singhasivanon P: Longitudinal study of Plasmodium falciparum and Plasmodium vivax in a Karen population in Thailand. Malar J 2008, 7: 99.

28. Teka H, Petros B, Yamuah L, Tesfaye G, Elhassan I, Muchohi S, Kokwaro G, Aseffa A, Engers H: Chloroquine-resistant Plasmodium vivax malaria in Debre Zeit, Ethiopia. Malar J 2008, 7: 220 . 


\section{Chapter III}

\section{Assessment of the prozone effect in malaria RDTs}

IIIa: Assessment of the prozone effect in malaria rapid diagnostic tests.

Gillet P, Mori M, Van Esbroeck M, Van den Ende J, Jacobs J.

Malaria Journal 2009; 8:271.

http://www.malariajournal.com/content/8/1/271

IIIb: Prozone in malaria rapid diagnostic test: how many cases are missed?

Gillet P, Scheirlinck A, Stokx J, De Weggheleire A, Chaúque $\mathrm{H}$, de Jesus Varela Canhanga O, Tadeu B, das Dores Mosse C, Tiago A, Mabunda S, Bruggeman C, Bottieau E, Jacobs J.

Submitted for publication. 


\section{Chapter IIIa}

\section{Assessment of the prozone effect in malaria RDTs}

IIIa: Assessment of the prozone effect in malaria rapid diagnostic tests.

Gillet P, Mori M, Van Esbroeck M, Van den Ende J, Jacobs J.

Malaria Journal 2009; 8:271.

http://www.malariajournal.com/content/8/1/271 


\title{
Assessment of the prozone effect in malaria rapid diagnostic tests
}

\begin{abstract}
Background

The prozone effect (or high dose-hook phenomenon) consists of false-negative or false-low results in immunological tests, due to an excess of either antigens or antibodies. Although frequently cited as a cause of false-negative results in malaria rapid diagnostic tests (RDTs), especially at high parasite densities of Plasmodium falciparum, it has been poorly documented. In this study, a panel of malaria RDTs was challenged with clinical samples with $P$. falciparum hyperparasitaemia (> 5\% infected red blood cells).
\end{abstract}

\section{Methods}

Twenty-two RDT brands were tested with seven samples, both undiluted and upon $10 \times, 50 \times$ and $100 \times$ dilutions in $\mathrm{NaCl} 0.9 \%$. The $P$. falciparum targets included histidine-rich protein-2 (HRP-2, $\mathrm{n}=17)$ and $P$. falciparum-specific parasite lactate dehydrogenase (Pf-pLDH, $\mathrm{n}=5)$. Test lines intensities were recorded in the following categories: negative, faint, weak, medium or strong. The prozone effect was defined as an increase in test line intensity of at least one category after dilution, if observed upon duplicate testing and by two readers.

\section{Results}

Sixteen of the 17 HRP-2 based RDTs were affected by prozone: the prozone effect was observed in at least one RDT sample/brand combination for $16 / 17 \mathrm{HRP}-2$ based RDTs in 6/7 samples, but not for any of the Pf-pLDH tests. The HRP-2 line intensities of the undiluted sample/brand combinations with prozone effect $(n=51)$ included a single negative (1.9\%) and 29 faint and weak readings $(56.9 \%)$. The other target lens ( $P$. vivax-pLDH, pan-specific-pLDH and aldolase) did not show a prozone effect.

\section{Conclusion}

This study confirms the prozone effect as a cause of false-negative HRP-2 RDTs in samples with hyperparasitaemia. 


\section{Background:}

Malaria rapid diagnostic tests (RDTs) are lateral flow immunochromatographic tests that detect Plasmodium antigens by antibody-antigen interactions on a nitrocellulose test strip. Capillary or venous blood and a lysis buffer are added to the strip: if present in the sample, the Plasmodium antigen is bound to a detection antibody. This detection antibody is usually a monoclonal mouse-antibody conjugated to a signal, mostly colloidal gold. The antigen-detection antibody-conjugate complex diffuses further across the strip until it is bound to a second antibody: this so-called capture antibody reacts to another epitope of the target antigen. As the capture antibody is fixed on a narrow section of the strip, the conjugated signal is concentrated and becomes visible as a cherry-red or purple coloured line. The excess of detection antibody-conjugate that was not bound by the antigen and the capture antibody moves further until it is bound to a goat anti-mouse antibody, thereby generating a control line. The Plasmodium antigens targeted by RDTs include those specific to Plasmodium falciparum (histidine-rich protein-2 (HRP-2) and P. falciparumspecific parasite lactate dehydrogenase (Pf-pLDH)), the antigen specific to Plasmodium vivax ( $P$. vivax-specific parasite lactate dehydrogenase, $\mathrm{Pv}-\mathrm{pLDH}$ ) and the antigens common to $P$. falciparum, $P$. vivax, Plasmodium ovale and Plasmodium malariae (pan-species parasite lactate dehydrogenase (pan-pLDH) and aldolase). The RDT strip is produced either as a simple dipstick or fixed in a cassette or cardboard format. RDTs combine a control line with one or more antigen detecting test lines: those with a single test line are named two band RDTs, those with two and three antigen test lines are known as three-band and four-band RDTs respectively.

The use of malaria rapid diagnostic tests is expanding, both in endemic and nonendemic settings [1]. In 2007, more than 70 million RDTs have been procured [2] and in many endemic countries, RDTs are now being rolled out as the instrument of choice for parasite-based malaria diagnosis and patient management at all levels of health care [3]. Under controlled conditions, RDTs have shown sensitivities close to $100 \%$ for the detection of $P$. falciparum, the most life-threatening species. However, there are still false-negative results: most of them occur at low parasite densities (< 100 asexual parasites/ $\mu 1$ or $<0.002 \%$ of red blood cells infected), but others occur at high parasite densities, in particular at hyperparasitaemia, defined by the World Health Organization (WHO) as infections with $>5 \%$ of red blood cells infected [4]. Mostly, the latter are described to genetic variations of the HRP-2 [3-5], but the prozone effect is also cited as an explanation [1,6-10]. The prozone effect (also known as high dose-hook phenomenon) is defined as false-negative or false-low results in immunological reactions, due to an excess of either antigens or antibodies [11]. It occurs particularly in one-step immunoassays, such as agglutination tests, for which serial dilutions are advised to trace the effect [12]. In the case of hyperparasitaemia in RDTs, high antigen concentrations will block all available 
binding sites of both the detection and the capture antibodies, thereby preventing the binding of the antigen-detection antibody-conjugate complex to the capture antibody, with failure of signal generation. Simple dilution of the sample will correct this effect. Despite frequently cited in literature on RDTs, there is only a single original report in which the prozone effect in RDT is unequivocally demonstrated by appearance of the test line upon dilution of the sample [13]. Since attention was drawn to this effect by a recent case (see below) presented at the Institute of Tropical Medicine (ITM), a panel of RDTs was challenged to clinical samples with $P$. falciparum hyperparasitaemia.

\section{Case description}

An EDTA-blood sample of a 69-year old male patient, returning from Nigeria was submitted to the reference laboratory of ITM. The referring laboratory had made the microscopic diagnosis of $P$. falciparum malaria with a parasite density of $27.5 \%$. However, they were puzzled about the result of the RDT which they performed in conjunction to microscopy: in addition to the control line, the test showed a clear pan-Plasmodium aldolase line but there was no HRP-2 line visible. According to the instructions of the manufacturer, this combination points to the diagnosis of nonfalciparum malaria.

Upon receipt of the sample, the diagnosis of $P$. falciparum was confirmed as well as the parasite density. The sample was tested with the usual panel of RDTs used in ITM. The BinaxNow ${ }^{\circledR}$ (Binax, Scarborough, Maine, U.S.) and the SD FK60 Malaria Ag P. falciparum/Pan (Standard Diagnostics, Hagal-Dong, Korea) showed faint and barely distinguishable HRP-2 lines in combination with clear control and panPlasmodium lines (aldolase and pan-pLDH respectively). The Optimal-IT test (DiaMed AG, Cressier s/Morat, Switzerland) showed clear lines for both Pf-pLDH and pan-pLDH. A $10 \times$ dilution of the EDTA-blood in $\mathrm{NaCl} 0.9 \%$ resulted for both the BinaxNow ${ }^{\circledR}$ and the SD FK60 tests in clearly distinguishable HRP-2 lines of intensities equal to the control line. When doubled volume of the undiluted blood was applied to the sample pad, the HRP-2 line was still visible as a faint line in the SD FK60, but was no longer visible in the BinaxNow ${ }^{\circledR}$ test. It was concluded that the prozone effect was the cause of the false-low and false-negative test line intensities.

\section{Methods}

\section{Patients' samples}

In this study EDTA-blood samples obtained from international travellers presenting at the outpatient clinic of ITM, Antwerp, Belgium, as well as samples submitted by Belgian laboratories to ITM in its functions as the National Reference Centre, were used. Part of them were fresh samples, the other samples had been stored at $-70^{\circ} \mathrm{C}$ 
till analysis. For all samples, the diagnosis of malaria was made by microscopy and confirmed with species-specific PCR as previously described [14]. Parasite density was assessed by counting the number of asexual parasites against 200 white blood cells in a thick film, and converting this value to parasites/ $\mu 1$ using the actual count or the standard of 8000 white blood cell/ $\mu 1$ [15]. For the purpose of this study, the more convenient parasite density as expressed in \% of infected red blood cells was applied, thereby assuming $50.000 / \mu 1$ to be equal to $1 \%$ of red blood cells parasitized [15]. P. falciparum-infected samples with hyperparasitaemia were selected and the sample with parasite density of $0.1 \%(5.000 / \mu l)$ was used as a control sample.

\section{Malaria rapid diagnostic tests}

Malaria RDTs chosen were those included in the WHO list of RDT manufacturers with adequate evidence of good manufacturing practice available online [16] and assessment was focused on those products available in cassette and folded card box format. In addition to this list, other RDTs available on the international market were randomly included. In view of the wide lot-to-lot variations and the ever changing composition of RDTs, it was decided not to display the individual RDT brand names, in line with a similar study that compared RDT heat stabilities [17].

Tests were performed according to the instructions of the manufacturer, except that samples were loaded with a pipette (Finnpipette, Helsinki, Finland) instead of the transfer device supplied by the manufacturer. In case the control line did not appear the result was interpreted as invalid and the test was repeated. In order to qualify the test line intensities, a scoring system of five categories was used: none (no line visible), faint (barely visible line), weak (paler than the control line), medium (equal to the control line) or strong (stronger than the control line) [14]. All tests were carried out in duplicate. Readings were performed by two readers at daylight assisted by a standard electric bulb, and within and not beyond the prescribed delay after application of the sample and buffer. Tests were performed on undiluted samples as well as on samples diluted $10 \times, 50 \times$ or $100 \times$ in saline solution $(\mathrm{NaCl})$ $0.9 \%$.

\section{Test outcomes and definitions}

For the results of test line intensities, consensus readings were considered, i.e. the line intensities most frequently scored in the duplicate test/two readers' combination. The prozone effect was defined as an increase in test line intensity of at least one category after dilution, if observed upon duplicate testing and by two readers. 


\section{Statistical analysis}

Inter-reader reliability for line intensities was calculated as percentage agreements and kappa values. Reproducibility was expressed as the consistency of line intensity readings for both readers and upon repeating the tests.

\section{Ethical review}

The study was reviewed and approved by the Institutional Review Board of ITM and by the Ethical Committee of Antwerp University, Belgium.

\section{Results}

\section{Collection of samples and RDTs}

Seven samples with hyperparasitaemia were elected, their parasite densities are listed in Table 1. Six samples had been stored at $-70^{\circ} \mathrm{C}$ for a period of three to 96 months, one was assessed freshly. They were all obtained in patients returning from sub-Saharan Africa. Twenty-two brands of RDTs were collected, 17 of them targeted $P$. falciparum by detecting HRP-2, the other five detected Pf-pLDH (two of which produced by the same company). The RDT brands included three two-band tests, 15 three-band tests and four four-band tests. The antigens targeting all four common species comprised pan-pLDH $(n=16)$ and aldolase $(n=2)$. Fourteen RDTs had CE mark compliance, 14 were included in the WHO list and one is authorized for use in the United States (US FDA approved).

\section{Inter-reader reliability and reproducibility}

Inter-reader reliability for P. falciparum test line intensities was high, with $86.7 \%$ agreement and a kappa value of 0.79 , and discrepancies limited to one category of line intensity (e.g. line intensity read as weak by reader 1 and as medium (but not strong) by reader 2). Upon duplicate testing, $82.5 \%$ and $80.3 \%$ of 319 P. falciparum line intensities were identically read by each of both readers respectively. For the non-falciparum test line intensities (pan-pLDH and aldolase), agreement and kappa value for line intensities between readers was $88.2 \%$ and 0.84 respectively. These differences had no effect on the numbers of samples with prozone effect. All 51 sample/brand combinations, (six samples for 12 brands) with prozone effect showed the effect as defined for both observers, and no additional cases were suggested by the observation of only a single observer.

\section{Prozone effect}

For the $\mathrm{Pv}$-pLDH, pan-pLDH and aldolase lines, there were 136 sample/brand combinations tested: in 51 (36.7\%) of them (representing 11/14 RDTs), the $10 \times$ diluted samples showed weaker line intensities as compared to the undiluted samples. The control lines were well visible in all cases, except in four invalid brand/sample combinations for a single brand (RDT nr. 4, Table 1). For the $P$. 
falciparum lines, there was a clear difference between HRP-2 lines and Pf-pLDH lines. For the control sample of parasite density of $0.1 \%$, all but one RDTs showed medium or strong line intensities upon undiluted testing; RDT nr.12 showed a weak HRP-2 line intensity. When assessed with the samples with hyperparasitaemia, the five three-band tests targeting Pf-pLDH did not show a prozone effect. For one RDT, there were two samples that showed a decrease in Pf-pLDH line intensity at the $10 \times$ dilution; the other combinations did not change line intensity upon dilution. By contrast, the prozone effect was observed for at least one sample in all but one HRP-2 RDT brands (Table 1). The single RDT brand that did not display a prozone effect was RDT nr. 12, which showed a decrease in test line intensity upon $10 \times$ dilution. The prozone effect tended to occur more frequently in particular brands and samples and was not directly related to the parasite count: for instance, the sample with $11.6 \%$ parasite density showed the prozone effect for all but one brand, whereas the sample with $35.0 \%$ parasite density did so for $8 / 21$ brands tested. There was no difference in prozone effect between RDTs that were CE-marked, FDA approved or WHO-listed and those which were not. In terms of test line intensities, the distribution for the undiluted sample/brand combinations with prozone effect (n $=51$ ) was as follows: a single $(1.9 \%)$ negative reading, four $(7.8 \%)$ faint readings, three (5.9\%) either faint or weak readings (depending on the observer), $22(43.1 \%)$ weak readings and $21(41.2 \%)$ medium readings. This means that for a total of 29 (56.9\%) of sample/brand combinations (in 12/17 RDTs) with the prozone effect, the undiluted sample showed a faint or weak HRP-2 line. Maximum (strong) line intensities were obtained at a $10 \times$ dilution for 28 out of 51 sample/brand combinations. For the remaining 23 combinations there was enough additional material for 16 combinations to perform $50 \times$ or $100 \times$ dilutions, in which 12 and four reached respectively the strong line intensity.

\section{Discussion}

RDTs offer great potential for the timely and accurate diagnosis of malaria, thereby leading to prompt and appropriate treatment. They have found their place in both malaria-endemic and non-endemic settings. In endemic settings, they offer parasitebased diagnosis in the absence of competent laboratory infrastructures as they can be carried out by non-specialized health care workers $[18,19]$. In non-endemic settings, where microscopic expertise is lacking due to low incidence, they are used as adjunct to microscopy especially outside office hours but also as bedside point of care tests [20,21]. In addition, RDTs are marketed for self-use by travellers [10]. In this study, RDTs were challenged with a panel of clinical samples with $P$. falciparum hyperparasitaemia. The prozone effect was observed for the HRP-2 test lines in 16/17 RDTs, and was consistent among the two observers. None of the $P$. falciparum specific Pf-LDH lines (tested in five RDTs) showed the prozone effect, nor did any of the pLDH and aldolase lines. 
The present study has its limitations. A calibrated pipette was used instead of the manufacturer's transfer device, thereby bypassing a possible error of the kit's application system. Next, in reference to the original report [13], dilutions were made in $\mathrm{NaCl} 0.9 \%$ and not, for instance, in the kit's diluent. Also, this evaluation was performed in a reference setting, with expert technicians who are used to evaluate RDTs and who are trained not to disregard faint positive lines, thereby possibly underestimating the incidence of the prozone effect as compared to field settings. Despite these limitations, this study documents the prozone effect among the present panel of RDTs according to stringent and reproducible criteria.

Most reviews and leading authorities point to the possibility of the prozone effect in RDTs $[1,8,22,23]$, but there is only a single original report describing this effect in a RDT: in 1999, Risch and co-workers described a patient returning from Yemen, with a $P$. falciparum infection at a parasite density of $30 \%$. The RDT they used (ICT Malaria, Pf, ICT, Australia - a HRP-2 targeted two band test which is no longer marketed) showed no test line for the undiluted sample, but a clearly distinguishable line at $10 \times$ dilution in $\mathrm{NaCl} 0.9 \%$ [13]. Another report described, as part of a prospective study, a patient returning from The Gambia, with microscopic diagnosis of $P$. falciparum at a parasite density of $31 \%$. For the BinaxNow ${ }^{\circledR}$ Malaria Pf/Pv test, the authors reported observations identical to those presently described, i.e. a faint HRP-2 line but a strong $P$. vivax-test line (the latter line representing in fact pan-Plasmodium LDH reactivity). Although the authors described this effect in full detail and added a picture, they did not refer to the possibility of the prozone effect and did not carry out dilution studies. In addition to these reports on hyperparasitaemia there are rare but consistent reports of unexplained failures of mainly HRP-2 RDTs at parasite densities in the intermediate ranges (e.g. between $10.000 / \mu 1$ and $100.000 / \mu 1(0.2 \%$ and $2 \%$ respectively [1,9,12,14,24-31]). The most frequently cited explanation for these failures is the presence of HRP-2 polymorphisms $[1,3,5,6,22]$, although the polymorphisms that are less likely to be picked up by RDTs are geographically confined to the Asia-Pacific region whereas many of the failures occurred in field settings in Africa [5]. The prozone effect in these samples can be an alternative explanation, but at present samples with intermediate parasite densities were not included in this study.

The observation that, among the presently studied RDTs, HRP-2 brands, but not PfpLDH brands are subject to the prozone effect is of interest but remains unexplained. Compared to the Pf-pLDH based RDTs, HRP-2 based RDTs also tend to be more affected by the rheumatoid factor, giving rise to false-positive results $[32,33]$. In addition, the absence of the prozone effect in the pan-pLDH and aldolase lines is in line with this observation. Although the prozone effect was observed in 
none of the currently tested Pf-pLDH based RDTs, further research should be done to confirm the absence of the prozone effect in other Pf-pLDH RDTs. It is also of note that the prozone effect did not occur in clear relation to the parasite density: this may be due to different factors affecting the circulating HRP-2 concentration, such as capillary sequestration of the parasites, variations in the production of antigen production during the cycle as well as by strain differences [34,35].

In the present study, only a single sample/brand combination showed complete absence of HRP-2 test line and four samples showed faint line intensities, representing only $5 / 51(9.8 \%)$ of the tests affected by prozone. However, three additional tests were read as faint by one out of two observers. Further, it should be stressed that the present readings were made by experienced technicians who were trained to interpret faint, weak and medium tests lines, and who were working in reference conditions. By contrast, misinterpretation of faint lines as negative results is a common mistake made by inexperienced staff, travellers and community health care workers both in endemic and no endemic settings [18,19,21,36] In endemic settings, readings of RDTs are frequently performed in unfavourable light conditions during evening and night shifts [37]. Finally it should be noted that the four faint lines all occurred in two of the three two-band RDTs that are frequently used in field setting.

The consequences of a falsely negative interpretation are serious: in the case of twoband tests, the diagnosis of malaria may be missed, and in the case of a three-band test, an infection with $P$. falciparum will be erroneously diagnosed as a nonfalciparum species. Of note is the observation with the submitted sample described in the case report: when the double sample volume was applied, there was complete absence of the HRP-2 test line in one of the RDTs. This may also cause problems in field settings, where there is a tendency to apply more than the required sample volume [38,39].

Although among the presently challenged panel the prozone effect was common, it is yet unclear how frequent it occurs in routine diagnosis. As to the use of RDTs in low resource settings, it is of note that all three tested HRP-2 two-band brands are used in high numbers by non-governmental organizations in emergency relief operations and are presently introduced in national malaria control programs [3]. For example, the 3,000,000 RDT tests provided in 2008 by the Global Fund to Fight AIDS, Tuberculosis and Malaria, all belonged to these three brands [40]. The impact of prozone for these three HRP-2 two-band brands is of concern as it occurred in two, four and six samples respectively. Moreover, the test line intensities obtained with undiluted samples were faint or weak in four and six samples respectively. 
In setting of ITM, samples with hyperparasitaemia occurred at a frequency of $5.5 \%$ among 200 single-patient $P$. falciparum samples processed over a 23 -month period (May 2007 - April 2009). Further study including incident reporting and large-scale prospective testing should be performed both in the endemic and the non-endemic settings in order to have reliable estimates of the prozone effect as a cause of falsenegative RDT results.

False-negative results at low parasite densities can be countered by diagnostic algorithms prescribing repeat or serial testing when malaria is suspected and the initial RDT test result is negative [6,12]. By contrast, false-negative results in hyperparasitaemias are to be corrected immediately. What can be done to prevent or overcome the prozone effect? First, it is imperative to train end-users of RDTs in correctly reading and interpreting faint and weak test lines and emphasizing that the appearance of the control line does not guarantee control of all aspects of RDT test performance. Second, for non-endemic settings, previous recommendations to perform competent microscopy in parallel to the RDTs should be emphasized [1]: an instructed lab technician, even when not expert in malaria and not able to perform species identification, is expected to find and recognize Plasmodium parasites in samples with hyperparasitaemia and even in samples with lower parasite densities. If microscopy is not possible or feasible, one could consider having a RDT targeting Pf-pLDH at the hand, thereby assuming that Pf-pLDH tests are not prone to the prozone effect. Suspected samples should be retested on a $10 \times$ and if needed a subsequent $50 \times$ dilution of the sample, with dilutions made in $\mathrm{NaCl} 0.9 \%$, pending further research on the most appropriate diluents. On the regulatory level, it would be interesting to test all marketed RDTs for their susceptibility to the prozone effect.

\section{Conclusions}

In conclusion, we demonstrated the prozone effect for the detection of $P$. falciparum in 16/17 HRP-2 based RDTs. The effect did not occur in the five Pf-pLDH RDTs neither in the pan-pLDH and aldolase lines. The HRP-2 line intensities in undiluted samples were negative in a single sample/brand combination and displayed faint or weak line intensities in nearly $60 \%$ of sample/brand combinations; therefore, the prozone effect is expected to have consequences in diagnosis and patient care both in endemic and non-endemic settings. Dilutions of $10 \times$ and if needed $50 \times$ should be made to detect this effect, and microscopy and/or a Pf-pLDH RDT can be done when the prozone effect is suspected. Further research should confirm the absence of the prozone effect in other Pf-pLDH targeted RDTs and assess the incidence of the prozone effect in false-negative results in both endemic and non-endemic settings. 


\begin{abstract}
Abbreviations
Ag: Antigen; CE: Conformité Européenne; EDTA: Ethylene diamine tetra-acetic acid; FDA: Food and drug administration; FHML: Faculty of Health Medicine and Life Sciences, Maastricht, The Netherlands; HRP-2: histidine-rich protein-2; ITM: Institute of Tropical Medicine; NaCl: Sodium chloride; P.: Plasmodium; Pf: Plasmodium falciparum; Pv: Plasmodium vivax; PCR: polymerase chain reaction; pan-pLDH: pan species parasite lactate dehydrogenase; Pf-pLDH: Plasmodium falciparum-specific parasite lactate dehydrogenase; $\mathrm{pLDH}$ : parasite lactate dehydrogenase; Pv-pLDH: Plasmodium vivax-specific parasite lactate dehydrogenase; RDT: Rapid diagnostic test; WHO: World Health Organization.
\end{abstract}

\title{
Acknowledgements
}

We would like to thank the laboratory staff of the Central Laboratory of Clinical Biology (ITM, Antwerp, Belgium) for technical support.

\section{References}

1. Marx A, Pewsner D, Egger M, Nuesch R, Bucher HC, Genton B, Hatz C, Juni, P: Metaanalysis: accuracy of rapid tests for malaria in travelers returning from endemic areas. Ann Intern Med 2005, 142: 836-846.

2. Bell D, Perkins MD: Making malaria testing relevant: beyond test purchase. Trans $R$ Soc Trop Med Hyg 2008, 102: 1064-1066.

3. Drakeley $\mathrm{C}$, Reyburn H: Out with the old, in with the new: the utility of rapid diagnostic tests for malaria diagnosis in Africa. Trans R Soc Trop Med Hyg 2009, 103: 333-337.

4. World Health Organization: World Health Organization. Severe falciparum malaria. Trans $R$ Soc Trop Med Hyg 2000, 94 (Suppl 1): 1-90.

5. Baker J, McCarthy J, Gatton M, Kyle DE, Belizario V, Luchavez J, Bell D, Cheng Q: Genetic diversity of Plasmodium falciparum histidine-rich protein 2 (PfHRP2) and its effect on the performance of PfHRP2-based rapid diagnostic tests. J Infect Dis 2005, 192: 870-877.

6. Farcas GA, Zhong KJ, Lovegrove FE, Graham CM, Kain KC: Evaluation of the Binax NOW ICT test versus polymerase chain reaction and microscopy for the detection of malaria in returned travelers. Am J Trop Med Hyg 2003, 69: 589-592.

7. Grobusch MP, Hanscheid T, Gobels K, Slevogt H, Zoller T, Rogler G, Teichmann D: Comparison of three antigen detection tests for diagnosis and follow-up of falciparum malaria in travellers returning to Berlin, Germany. Parasitol Res 2003, 89: 354-357.

8. Hawkes M, Kain KC: Advances in malaria diagnosis. Expert Rev Anti Infect Ther 2007, 5: 485495.

9. Pieroni P, Mills CD, Ohrt C, Harrington MA, Kain KC: Comparison of the ParaSight-F test and the ICT Malaria Pf test with the polymerase chain reaction for the diagnosis of Plasmodium falciparum malaria in travellers. Trans $R$ Soc Trop Med Hyg 1998, 92: 166-169.

10. Risch L, Bader M, Huber AR: Self-use of rapid tests for malaria diagnosis. Lancet 2000, 355: 237.

11. Heidelberger M, Kendall F: a quantitative therory of the precipitin reaction. J Exp Med 1935, 62: $697-720$.

12. Forney JR, Magill AJ, Wongsrichanalai C, Sirichaisinthop J, Bautista CT, Heppner DG, Miller RS, Ockenhouse CF, Gubanov A, Shafer R, DeWitt C, Quino-Ascurra HA, Kester KE, Kain KC, Walsh DS, Ballou WR, Gasser RAJr: Malaria rapid diagnostic devices: performance characteristics of the ParaSight $\mathbf{F}$ device determined in a multisite field study. $J$ Clin Microbiol 2001, 39: 2884-2890.

13. Risch L, Bader M, Huber AR: False negative quick malaria test. Schweiz Med Wochenschr 1999, 129: 1002. 
14. Van der Palen M., Gillet P, Bottieau E, Cnops L, Van Esbroeck M., Jacobs J: Test characteristics of two rapid antigen detection tests (SD FK50 and SD FK60) for the diagnosis of malaria in returned travellers. Malar J 2009, 8: 90.

15. Moody AH, Chiodini PL: Non-microscopic method for malaria diagnosis using OptiMAL IT, a second-generation dipstick for malaria pLDH antigen detection. Br J Biomed Sci 2002, 59: 228-231.

16. World Health Organization. List of known commercially-available antigen-detecting malaria RDTs with adequate evidence of good manufacturing practice. 2009. [http://www.wpro.who.int/NR/rdonlyres/E3597CBA-2ED4-4FCC-AB1CCE25FC6AA30E/0/M D Webbuy Table9.pdf]

17. Chiodini PL, Bowers K, Jorgensen P, Barnwell JW, Grady KK, Luchavez J, Moody AH, Cenizal A, Bell D: The heat stability of Plasmodium lactate dehydrogenase-based and histidine-rich protein 2-based malaria rapid diagnostic tests. Trans $R$ Soc Trop Med Hyg 2007, 101: 331337.

18. Mayxay M, Newton PN, Yeung S, Pongvongsa T, Phompida S, Phetsouvanh R, White NJ: Short communication: An assessment of the use of malaria rapid tests by village health volunteers in rural Laos. Trop Med Int Health 2004, 9: 325-329.

19. Rennie W, Phetsouvanh R, Lupisan S, Vanisaveth V, Hongvanthong B, Phompida S, Alday P, Fulache M, Lumagui R, Jorgensen P, Bell D, Harvey S: Minimising human error in malaria rapid diagnosis: clarity of written instructions and health worker performance. Trans $R$ Soc Trop Med Hyg 2007, 101: 9-18.

20. Chilton D, Malik AN, Armstrong M, Kettelhut M, Parker-Williams J, Chiodini PL: Use of rapid diagnostic tests for diagnosis of malaria in the UK. J Clin Pathol 2006, 59: 862-866.

21. Wiese L, Bruun B, Baek L, Friis-Moller A, Gahrn-Hansen B, Hansen J, Heltberg O, Hojbjerg T, Hornstrup MK, Kvinesdal B, Gomme G, Kurtzhals JA: Bedside diagnosis of imported malaria using the Binax Now malaria antigen detection test. Scand J Infect Dis 2006, 38: 1063-1068.

22. Erdman LK, Kain KC: Molecular diagnostic and surveillance tools for global malaria control. Travel Med Infect Dis 2008, 6: 82-99.

23. World Health Organization.. Regional Office for the Western Pacific 2003. Malaria Rapid Diagnosis: Making it Work. Meeting report 20-23 January 2003. Manila, the Philippines. 2003. [http://www.searo.who.int/LinkFiles/Malaria_MalariaRDT.pdf]

24. Gaye O, Diouf M, Dansokho EF, McLaughlin G, Diallo S: Diagnosis of Plasmodium falciparum malaria using ParaSight F, ICT malaria PF and malaria IgG CELISA assays. Parasite 1998, 5: 189-192.

25. Humar A, Ohrt C, Harrington MA, Pillai D, Kain KC: Parasight F test compared with the polymerase chain reaction and microscopy for the diagnosis of Plasmodium falciparum malaria in travelers. Am J Trop Med Hyg 1997, 56: 44-48.

26. Huong NM, Davis TM, Hewitt S, Huong NV, Uyen TT, Nhan DH, Cong leD: Comparison of three antigen detection methods for diagnosis and therapeutic monitoring of malaria: a field study from southern Vietnam. Trop Med Int Health 2002, 7: 304-308.

27. Jelinek T, Grobusch MP, Schwenke S, Steidl S, von Sonnenburg F, Nothdurft HD, Klein E, Loscher T: Sensitivity and specificity of dipstick tests for rapid diagnosis of malaria in nonimmune travelers. J Clin Microbiol 1999, 37: 721-723.

28. Kyabayinze DJ, Tibenderana JK, Odong GW, Rwakimari JB, Counihan H: Operational accuracy and comparative persistent antigenicity of HRP2 rapid diagnostic tests for Plasmodium falciparum malaria in a hyperendemic region of Uganda. Malar J 2008, 7: 221.

29. Ohrt C, Obare P, Nanakorn A, Adhiambo C, Awuondo K, O'Meara WP, Remich S, Martin K, Cook E, Chretien JP, Lucas C, Osoga J, McEvoy P, Owaga ML, Odera JS, Ogutu B: Establishing a malaria diagnostics centre of excellence in Kisumu, Kenya. Malar J 2007, 6: 79.

30. Stow NW, Torrens JK, Walker J: An assessment of the accuracy of clinical diagnosis, local microscopy and a rapid immunochromatographic card test in comparison with expert microscopy in the diagnosis of malaria in rural Kenya. Trans $R$ Soc Trop Med Hyg 1999, 93: 519-520.

31. Wongsrichanalai C, Chuanak N, Tulyayon S, Thanoosingha N, Laoboonchai A, Thimasarn K Brewer TG, Heppner $D G$ : Comparison of a rapid field immunochromatographic test to expert microscopy for the detection of Plasmodium falciparum asexual parasitemia in Thailand. Acta Trop 1999, 73: 263-273. 
32. Craig MH, Bredenkamp BL, Williams CH, Rossouw EJ, Kelly VJ, Kleinschmidt I, Martineau A, Henry GF: Field and laboratory comparative evaluation of ten rapid malaria diagnostic tests. Trans R Soc Trop Med Hyg 2002, 96: 258-265.

33. Jelinek T, Schulte C, Behrens R, Grobusch MP, Coulaud JP, Bisoffi Z, Matteelli A, Clerinx J, Corachan M, Puente S, Gjorup I, Harms G, Kollaritsch H, Kotlowski A, Bjorkmann A, Delmont J.P, Knobloch J, Nielsen LN, Cuadros J, Hatz C, Beran J, Schmid ML, Schulze M, Lopez-Velez R, Fleischer K, Kapaun A, McWhinney P, Kern P, Atougia J, Fry G, da Cunha S, Boecken G: Imported Falciparum malaria in Europe: sentinel surveillance data from the European network on surveillance of imported infectious diseases. Clin Infect Dis 2002, 34: 572-576.

34. Murray CK, Gasser RA, Jr., Magill AJ, Miller RS: Update on rapid diagnostic testing for malaria. Clin Microbiol Rev 2008, 21: 97-110.

35. Peeling RW, Smith PG, Bossuyt PM: A guide for diagnostic evaluations. Nat Rev Microbiol 2006, 4: S2-S6.

36. Trachsler M, Schlagenhauf $P$, Steffen R: Feasibility of a rapid dipstick antigen-capture assay for self-testing of travellers' malaria. Trop Med Int Health 1999, 4: 442-447.

37. Harvey SA, Jennings L, Chinyama M, Masaninga F, Mulholland K, Bell DR: Improving community health worker use of malaria rapid diagnostic tests in Zambia: package instructions, job aid and job aid-plus-training. Malar J 2008, 7: 160.

38. McMorrow ML, Masanja MI, Abdulla SM, Kahigwa E, Kachur SP: Challenges in routine implementation and quality control of rapid diagnostic tests for malaria--Rufiji District, Tanzania. Am J Trop Med Hyg 2008, 79: 385-390.

39. Moonasar D, Goga AE, Frean J, Kruger P, Chandramohan D: An exploratory study of factors that affect the performance and usage of rapid diagnostic tests for malaria in the Limpopo Province, South Africa. Malar J 2007, 6: 74.

40. The Global Fund to Fight AIDS, Tuberculosis and Malaria: PRM Full Purchase Price Report. 2008. [http://pqr.theglobalfund.org/PQRWeb/Templates/PRM Full Purchase Price Report.xls] 


\section{Chapter IIla: Assessment of the prozone effect in Malaria RDTs}

Table1: Prozone effect in 17 HRP-2 based malaria rapid diagnostic tests. Prozone effect was defined as an increase in HRP-2 line intensity upon dilution of the sample as observed in two tests and read by two observers. In case of prozone effect, line intensities for the undiluted samples are recorded in the Table, they are categorized as Negative,

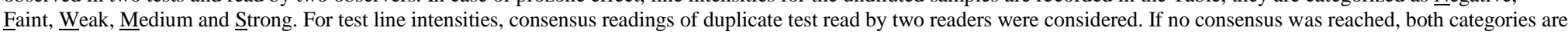
listed.

\begin{tabular}{|c|c|c|c|c|c|c|c|c|}
\hline \multicolumn{2}{|r|}{ Malaria RDT } & \multicolumn{7}{|c|}{ Samples with hyperparasitemia and origin } \\
\hline & & $5.5 \%$ & $6.3 \%$ & $9.0 \%$ & $11.6 \%$ & $17 \%$ & $27.5 \%$ & $35.0 \%$ \\
\hline $\mathrm{Nr}$ & Type (other antigen detection) & Ivory Cost & DRC & Tanzania & Togo & Nigeria & Nigeria & Benin \\
\hline 1 & Two-band & & & & M & & M & \\
\hline 2 & Two-band & $\mathrm{F}^{\mathrm{a}}$ & & & $\mathrm{W}$ & & $\mathrm{W}^{\mathrm{a}}$ & $\mathrm{W}^{\mathrm{a}}$ \\
\hline 3 & Two-band & $\mathrm{W}^{\mathrm{a}, \mathrm{b}}$ & & $\mathrm{F}^{\mathrm{a}, \mathrm{b}}$ & $\mathrm{F}^{\mathrm{a}, \mathrm{b}}$ & $\mathrm{W}^{\mathrm{a}}$ & $\mathrm{F}$ & $\mathrm{W}^{\mathrm{a}, \mathrm{b}}$ \\
\hline 4 & Three-band (aldolase) & & Invalid & Invalid & $\mathrm{N}^{\mathrm{a}}$ & Invalid & Invalid & $\mathrm{W}$ \\
\hline 5 & Three-band (aldolase) & M & & M & $\mathrm{W}^{\mathrm{a}, \mathrm{b}}$ & & & $\mathrm{W}^{\mathrm{a}, \mathrm{b}}$ \\
\hline 6 & Three-band (pan-pLDH) & & & & $\mathrm{W}^{\mathrm{a}, \mathrm{b}}$ & M & $\mathrm{W}^{\mathrm{a}, \mathrm{b}}$ & \\
\hline 7 & Three-band (pan-pLDH) & & & & M & & M & \\
\hline 8 & Three-band (pan-pLDH) & & & $\mathrm{M}$ & $\mathrm{W}$ & & $\mathrm{W}^{\mathrm{a}, \mathrm{b}}$ & $\mathrm{M}$ \\
\hline 9 & Three-band (pan-pLDH) & & & $\mathrm{W}$ & $\mathrm{W}^{\mathrm{a}, \mathrm{b}}$ & $\mathrm{M}$ & $\mathrm{W}^{\mathrm{a}} / \mathrm{F}^{\mathrm{a}}$ & $\mathrm{M}$ \\
\hline 10 & Three-band (pan-pLDH) & & & $\mathrm{M}$ & $\mathrm{W}^{\mathrm{a}, \mathrm{b}}$ & $\mathrm{M}$ & $\mathrm{W}^{\mathrm{a} / \mathrm{F}^{\mathrm{a}}}$ & $\mathrm{W}^{\mathrm{a}, \mathrm{b}}$ \\
\hline 11 & Three-band (pan-pLDH) & & & & $\mathrm{M}$ & & $\mathrm{M}$ & \\
\hline 12 & Three-band (pan-pLDH) & & & & & & & \\
\hline 13 & Three-band (Pv-pLDH) & $\mathrm{M}$ & & $\mathrm{W}^{\mathrm{a}}$ & $\mathrm{W} / \mathrm{F}$ & $\mathrm{M}$ & ND & ND \\
\hline 14 & Four-band (Pv-pLDH, pan-pLDH) & & & & $\mathrm{W}$ & & $\mathrm{M}^{\mathrm{b}}$ & \\
\hline 15 & Four-band (Pv-pLDH, pan-pLDH) & & & $\mathrm{M}^{\mathrm{b}}$ & $\mathrm{W}^{\mathrm{a}}$ & & & \\
\hline 16 & Four-band (Pv-pLDH, pan-pLDH) & & & ND & $\mathrm{W}^{\mathrm{a}, \mathrm{b}}$ & $\mathrm{M}$ & ND & $\mathrm{M}^{\mathrm{b}}$ \\
\hline 17 & Four-band (Pv-pLDH, pan-pLDH) & & & ND & $\mathrm{W}^{\mathrm{a}}$ & & $\mathrm{ND}$ & \\
\hline
\end{tabular}

Invalid: invalid test result: no appearance of a control line.

ND: not performed because the sample or the Malaria RDT kit were exhausted.

a: test line intensity increased with 2 categories after dilution; in other cases, test line intensity increased with one category.

${ }^{\mathrm{b}}$ : maximum dilution obtained at $50 \times$ or $100 \times$ dilution; in other cases maximum line intensity was obtained at $10 \mathrm{x}$ dilution. 


\section{Chapter IIIb}

\section{Assessment of the prozone effect in malaria RDTs}

IIIb: Prozone in malaria rapid diagnostic test: how many cases are missed?

Gillet P, Scheirlinck A, Stokx J, De Weggheleire A, Chaúque H, de Jesus Varela Canhanga O, Tadeu B, das Dores Mosse C, Tiago A, Mabunda S, Bruggeman C, Bottieau E, Jacobs J

Submitted for publication 


\title{
Prozone in malaria rapid diagnostics tests: how many cases are missed?
}

\begin{abstract}

\section{Background}

Prozone means false-negative or false-low results in antigen-antibody reactions, due to an excess of either antigen or antibody. The present study prospectively assessed its frequency for malaria rapid diagnostic tests (RDTs) and Plasmodium falciparum samples in an endemic field setting.
\end{abstract}

\section{Methods}

From January to April 2010, blood samples with $P$. falciparum high parasitemia ( $\geq 4$ $\%$ red blood cells infected) were obtained from patients presenting at the Provincial Hospital of Tete (Mozambique). Samples were tested undiluted and 10-fold diluted in saline with a panel of RDTs and results were scored for line intensity (no line visible, faint, weak, medium and strong). Prozone was defined if a sample showed no visible test line or a faint or weak test line when tested undiluted, and a visible test line of higher intensity when tested $10 \times$ diluted, as observed by two blinded observers and upon duplicate testing.

\section{Results}

A total of $873 / 7,543(11.6 \%)$ samples showed $P$. falciparum, $92(10.5 \%)$ had high parasitemia and 76 were available for prozone testing. None of the two Pf-pLDH RDTs but all six HRP-2 RDTs showed prozone, at frequencies between $6.7 \%$ and $38.2 \%$. Negative and faint HRP-2 lines accounted for $4(3.8 \%)$ and $15(14.4 \%)$ of the 104 prozone results in two RDT brands. For the most affected brand, the proportions of prozone with no visible or faint HRP-2 lines were 10.9\% (CI: 5.3419.08), 1.2\% (CI: 0.55-2.10) and 0.1\% (CI: 0.06-0.24) among samples with high parasitemia, all positive samples and all submitted samples respectively. Prozone occurred mainly but not exclusively among young children.

\section{Conclusion}

Prozone occurs at different frequency and intensity in HRP-2 RDTs and may decrease diagnostic accuracy in the most affected RDTs. 


\section{Background}

Current malaria rapid diagnostic tests (RDTs) detect Plasmodium antigens in blood by antibody-antigen interactions on a nitrocellulose test strip. The targeted antigens include those specific to Plasmodium falciparum (histidine-rich protein-2 (HRP-2) and $P$. falciparum-specific parasite lactate dehydrogenase (Pf-pLDH)) and antigens common to P. falciparum, Plasmodium vivax, Plasmodium ovale and Plasmodium malariae (pan-species pLDH and aldolase). RDTs combine a control line with one, two or three antigen-detecting test lines, and are referred to as two-, three- and fourband RDTs respectively.

RDTs are being rolled out as an alternative to microscopic diagnosis in malaria endemic settings [1] and have demonstrated sensitivities close to $100 \%$ for the detection of $P$. falciparum at densities above 100 asexual parasites $/ \mu 1$ or $>0.002 \%$ of parasitized red blood cells (RBC). Most false-negative results occur at lower parasite densities. However, false-negative results have been reported also at high parasite densities. Part of those may be ascribed to genetic variations of the HRP-2 [2-6], but the prozone phenomenon may also be involved. Prozone is defined as false-negative or false-low results in antigen-antibody immunological reactions, due to an excess of either antigens or antibodies [7,8]. In RDTs, the prozone has been observed in samples with high $P$. falciparum parasite densities and dilution of the sample can trace and correct the effect [9]. In a recent laboratory evaluation, RDT brands were challenged to a panel of clinical samples with $P$. falciparum. Prozone was observed among 16 out of 17 HRP-2 RDTs but not among five Pf-pLDH RDTs [9]. However, as this was a retrospective laboratory study, the frequency and diagnostic impact of the prozone effect in daily practice remained unclear.

The main aim of the present study was to assess the frequency of the prozone effect in a malaria endemic field setting. A subsidiary aim was the confirmation of the previous observation that HRP-2 RDTs, but not Pf-pLDH tests, are affected by prozone [9].

\section{Methods}

\section{Study site, study period and patients included}

The study was conducted in the emergency ward of the Provincial Hospital of Tete (PHT), located in Central Mozambique. In this area, malaria is predominantly caused by $P$. falciparum. Transmission is perennial with peaks during and at the end of the rainy season (February - April)[10,11].

The PHT serves as a reference hospital for Tete Province (1,700,000 inhabitants). According to hospital statistics, yearly approximately 50.000 patients present with 
clinical suspicion of malaria. Diagnosis is confirmed in about $20 \%$ of them (either by RDT or microscopy). For this study, all patients suspected of malaria and presenting at the emergency ward of the PHT were prospectively included on a 7 days/24 hours basis from January to April 2010.

\section{Patients, samples and diagnostic work-up}

Routine procedures for malaria diagnosis (following national guidelines) at PHT are as follows: EDTA-anticoagulated blood is sampled and a full blood count is performed by an automated hematology analyzer (KX-21N, Sysmex, Kobe, Japan). For children $\leq 5$ years, malaria diagnosis is made on a thick blood film (TBF). For patients above 5 years of age, an RDT is performed and in case of a positive result a TBF is made for confirmation and determination of parasite density. TBFs are stained for 20 minutes at pH 7.2 using Giemsa 3.5\% (Merck, KGmA, Darmstadt, Germany). According to the national malaria clinical guidelines, parasite density is scored on a semi-quantitative scale from 1+ (1-9 asexual parasites/100 high power microscopic fields) to $5+$ (> 100 asexual parasites/1 high power microscopic field) [12]. During the study period, three RDT brands (ICT Malaria, Paracheck-Pf and $S D$ Malaria Antigen $P f F K 50$ ) were routinely used (provided by the national malaria program or a partner NGO).

For the purpose of the study, demographic data, presenting symptoms and clinical signs of malaria severity [13] were recorded for all patients attending the PHT with clinical suspicion of malaria. In addition, TBFs were performed for all suspected patients, irrespective of their age. For TBFs positive for P. falciparum and scored as $4+$ or 5+, parasite densities were quantitatively assessed: the number of asexual parasites was counted against 200 white blood cells (WBC) and converted to parasites $/ \mu 1$ using the WBC count $/ \mu 1$. Hereafter values were converted to $\%$ of

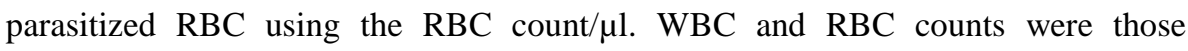
provided by the hematology analyzer.

Samples with a high parasitemia, defined as $\geq 4 \%$ of parasitized RBC [14,15], were challenged against a panel of RDTs consisting of four HRP-2 RDTs and two PfpLDH RDTs (see below and Table 1). Determination of parasite density and testing of RDTs were done at the latest 48 hours after sampling and samples were stored at $4^{\circ} \mathrm{C}$ pending RDT testing. Analyses were performed by the regular laboratory staff as well as the authors PG, AS, JS and HC. Left-overs of the EDTA blood samples were stored at $-20^{\circ} \mathrm{C}$ till the end of the study for further analyses. 
Table 1: Panel of RDT brands used in the study.

Brands / manufacturers and

Lot numbers

\section{ICT Malaria}

ICT Diagnostics, Cape Town, South

Africa

Lot $\mathrm{n}^{\circ}: 32784$

Paracheck-Pf

Orchid Biomedical Systems, Goa, India

Lot $n^{\circ}$ : A31003, 31672, 32972, 31797

SD Malaria Antigen Pf FK50

Standard Diagnostics, Hagal-Dong, Korea

Lot $\mathrm{n}^{\circ}$ : 082011, 082012, 080216

SD Malaria Ag Pf/Pan FK60

Standard Diagnostics, Hagal-Dong, Korea

Lot $\mathrm{n}^{\circ}:$ 090008, 090010, 090026

Hexagon Malaria Combi ${ }^{\ddagger}$

Human Wiesbaden, Germany

Lot $n^{\circ}: 80930$

Malaria Pan/Pv/Pf Rapid Device

Biotec laboratories Ltd., Ipswitch, UK

Lot $\mathrm{n}^{\circ}: 91081,91100$

CareStart Malaria $\mathrm{pLDH}$

Acces Bio, New Jersey, USA

Lot $n^{\circ}:$ B191L, A101L

SD Malaria pLDH FK40

Standard Diagnostics, Hagal-Dong, Korea

Lot $n^{\circ}: 081006$

First Response Malaria Ag Combo

Premier Medical Coorporation Ltd.,

Daman, India

Lot $\mathrm{n}^{\circ}: 6900309$

Hexagon Malaria

Human, Wiesbaden, Germany

Lot $\mathrm{n}^{\circ}: 0001$

ICT Malaria Combo $\$$

ICT Diagnostics, Cape Town, South

Africa

Lot $\mathrm{n}^{\circ}: 32250$

Malaria Total Quick ${ }^{\$}$

Cypress Diagnostics, Leuven, Belgium

Lot $n^{\circ}: 090002$

Format and
Plasmodium antigens
targeted ( $P$. falciparum
target is underlined)

Two-band: $\underline{\text { HRP-2 }}$

Two-band: HRP-2

Two-band: $\underline{\text { HRP-2 }}$

Three-band: HRP-2,

pan-pLDH

Three-band: $\underline{\text { HRP-2, }}$

aldolase

WHO FIND
Procurement
list $^{*}$

WHO FIND

Evaluation $^{\dagger}$

yes

yes

yes

yes

yes

yes

Four-band: HRP-2,

Pan-pLDH, Pv-pLDH

no

no

Three-band: Pf-pLDH,

pan-pLDH

no

yes

Three-band: Pf-pLDH, pan-pLDH

no

yes

Three-band: HRP-2,

Pan-pLDH

yes

yes

Two-band:: HRP-2

no

yes

Three-band: HRP-2,

Aldolase

no

yes

Three-band:HRP-2,

Pan-PLDH no

no

\footnotetext{
Included in the interim selection for procurement of malaria rapid diagnostic test [43].

†'Evaluated by WHO/FIND [16,17].

RDTs assessed for prozone on stored samples at the end of the study.

$\$$ Additional panel of RDTs assessed at the end of the study with a subset of stored samples positive for prozone for at least one of the prospectively assessed HRP-2 RDTs.
} 


\section{Malaria rapid diagnostic tests used and test procedures}

Malaria RDTs in cassette format were selected based on demonstrated diagnostic accuracy [16-18], use by national malaria control programs and non-governmental organizations (NGOs) and availability in Mozambique.

For each sample with high parasitemia, a 10-fold dilution was made in saline. Both undiluted and diluted sample were assessed with each RDT brand and tests were run in duplicate and read by two observers. The second observer was blinded to the first observer's readings. Tests were performed according to the instructions of the manufacturer, except for the use of an automatic pipette (Finnpipette, Helsinki, Finland) instead of the RDT kits' transfer device. Test line intensities were scored into five categories: none (no line visible), faint (barely visible), weak (paler than the control line), medium (equal to the control line) and strong (stronger than the control line) [19]. When a control line did not appear, the test was interpreted as invalid and the sample was retested. To assure timely readings, tests were performed in timecontrolled batches. Readings were carried out at daylight, within the prescribed reading delay.

\section{Additional analyses}

Two additional HRP-2 RDT brands (Hexagon Malaria Combi and Malaria Pan/Pv/Pf Rapid device), not delivered in time for the prospective analysis, were assessed at the end of the study period with samples that had been stored at $-20^{\circ} \mathrm{C}$ (maximum storage duration: 121 days).

In order to investigate the prozone occurrence at parasite densities below $4 \%$, all RDT brands were also assessed with a subset of 45 samples scored as $4+$ or $5+$ but with parasite densities below $4 \%$.

To confirm the prozone susceptibility of HRP-2 RDTs, an additional panel of four HRP-2 based RDTs was assessed with a subset of stored samples that had demonstrated prozone for at least one of the prospectively assessed HRP-2 RDTs (Table 1). Among the evaluated RDTs, there was also an additional lot number of Malaria Pan/Pv/Pf Rapid Device. The results of these latter panels were not included for calculation of the frequency of prozone.

\section{Test outcomes and definitions}

Samples with high parasitemia were defined as samples with parasite densities $\geq$ $4 \%$. Prozone was defined if a sample showed no visible test line or a faint or weak test line when tested undiluted, and a visible test line of higher intensity when tested 10 xdiluted, as observed by two blinded observers and upon duplicate testing [9]. 
The frequency of prozone was extrapolated against the total number of samples with high parasitemia, the total number of $P$. falciparum positive samples and the total number of patients suspected of malaria.

\section{Quality control}

At the start of the study, the laboratory staff received a refresher course on malaria microscopy and RDTs. The study team was trained on study procedures and flow during a pilot phase.

All RDTs were purchased in Belgium and shipped to Tete, except for $S D$ malaria Antigen Pf FK 50 and the lot A31003 of the Paracheck-Pf which were provided locally. During shipment and storage, temperature and humidity were monitored using loggers (Ebro Electronic GmBH, Ingolstadt, Germany). On a daily basis, 10\% of TBFs were randomly elected and reread by a member of the study team who was blinded to the original result. All discordances were resolved by a third reader's reading. A photograph was taken from all RDT tests performed and the TBFs were stored for quality control.

\section{Data management and statistical analysis}

According to the initial sample size calculation, a total of 5,700 patients suspected of malaria were required for reliable estimation of the frequency of prozone. This was based on the following assumptions and information from hospital statistics: malaria-attributable fraction during the wet season of 30\%, prevalence of hyperparasitemia of $10 \%$, prozone frequency among hyperparasitemia samples of $30 \%$ with $95 \%$ confidence intervals (CI) between $20 \%$ and $40 \%$.

Data were recorded in registers and on individual case report forms. Data were entered in Microsoft Access (Microsoft Corporation, Redmond, Washington USA) and analyzed in Stata 11.1 (StataCorp LP, College Station, USA). Differences between proportions were tested for significance using the Pearson's Chi-square test or, in case of small sample sizes, a two tailed Fisher's exact test. Reproducibility and inter-observer reliability for line intensity readings were assessed using the kappa statistic for paired observers and percentage agreements. Differences between medians were tested using the Wilcoxon test. Lot variations for matched pairs of samples were assessed using the McNemar's test. The relation between line intensities of prozone samples across the parasite densities was assessed using the Cuzick's test for trend. A p-value $<0.05$ was considered as significant. 


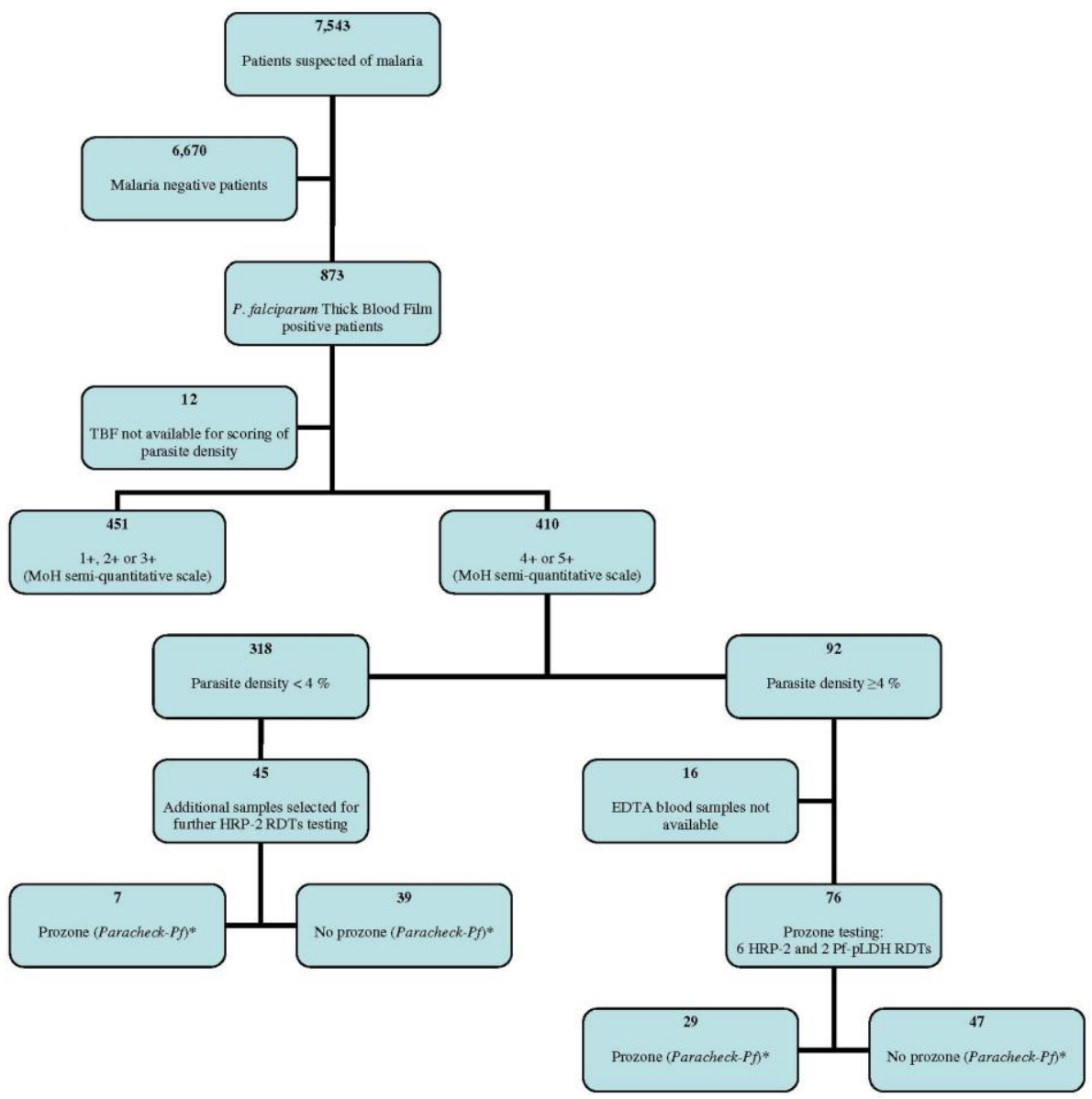

Figure 1: Flow of patients and samples.

* Prozone results presented for Paracheck-Pf, results for other RDTs are listed in Table 3. For definition of prozone see text.

\section{Ethical review}

The study was approved by the Institutional Review Board of ITM, by the ethical committee of Antwerp University, Belgium and by the Comité Nacional de bioética para a Saude $(\mathrm{MoH})$, Mozambique. Patients, children's parents or guardians were informed in Portuguese or in the local language (Nhungue) and their written consent was required prior to enrolment.

\section{Results}

\section{Patients, samples included and flow chart of the study}

During the study period, a total of 7,543 patients with suspicion of malaria were included, of whom $873(11.6 \%)$ were diagnosed with $P$. falciparum infection by 
thick blood film (Figure 1). About half of the positive patients with available TBF had parasite densities of $4+$ or $5+(410 / 861,47.6 \%)$ and 92 had parasite densities $\geq 4$ $\%$. Table 2 summarizes the demographic and parasitological characteristics of malaria positive patients with complete available data.

Table 2: Characteristics of $P$. falciparum samples included.

\begin{tabular}{|c|c|c|c|c|c|}
\hline \multirow{2}{*}{$\begin{array}{l}\text { Parasite density } \\
\text { (MoH semi- } \\
\text { quantitative scale) }\end{array}$} & \multicolumn{5}{|c|}{ Age group (nrs) } \\
\hline & $\begin{array}{l}\text { Children } \\
0-5 \text { years }\end{array}$ & $\begin{array}{l}\text { Children } \\
5-14 \text { years }\end{array}$ & $\begin{array}{c}\text { Adults } \\
(\geq 15 \text { years })\end{array}$ & $\begin{array}{l}\text { No } \\
\text { data }\end{array}$ & Total \\
\hline $1+$ & 39 & 19 & 97 & 12 & 167 \\
\hline $2+$ & 24 & 18 & 85 & 11 & 138 \\
\hline $3+$ & 27 & 20 & 91 & 8 & 146 \\
\hline $4+$ & 81 & 40 & 110 & 9 & 240 \\
\hline $5+$ & 97 & 32 & 36 & 5 & 170 \\
\hline Total & 268 & 129 & 419 & 45 & 861 \\
\hline
\end{tabular}

\section{Quality assessment, invalid test results, reproducibility and inter-observer reliability}

Shipment and storage temperatures of RDTs ranged between $4.0^{\circ} \mathrm{C}$ and $23.5^{\circ} \mathrm{C}$ (mean $17.5 \pm 8.7^{\circ} \mathrm{C}$ ) and $23.3^{\circ} \mathrm{C}$ and $32.3^{\circ} \mathrm{C}$ (mean $28.9 \pm 1.5^{\circ} \mathrm{C}$ ) respectively. Storage temperatures exceeded the highest allowed temperature $\left(30^{\circ} \mathrm{C}\right)$ for three RDT brands Hexagon Malaria Combi, Malaria Pan/Pv/Pf Rapid Device and CareStart Malaria $p L D H)$ : temperatures of $31^{\circ} \mathrm{C}$ and $32^{\circ} \mathrm{C}$ were registered for a cumulative period of 7.6 and 1 days respectively. Invalid results were observed for five brands and ranged between $0.2 \%$ and $0.7 \%$ of tests performed; upon repetition all tests performed well. For the HRP-2 line intensity readings, the overall agreement among the two observers ranged from $86.1 \%$ (Paracheck-Pf) to $97.1 \%$ (SD malaria Ag Pf/ Pan FK 60) and kappa values ranged from 0.67 (Malaria $\mathrm{Pan} / \mathrm{Pv} / \mathrm{Pf}$ rapid device) to 0.90 (ICT Malaria). The reproducibility of the RDTs among the duplicate tests in terms of HRP-2 line intensity ranged from $71.7 \%$ (Hexagon Malaria Combi) to $97.4 \%$ (SD malaria Ag Pf/ Pan FK 60) and kappa values ranged from 0.5 (Hexagon Malaria Combi) to 0.8 (SD malaria Ag Pf/ Pan FK 60).

\section{Frequency of the occurrence of prozone}

Prozone affected all six HRP-2 RDT brands in proportions ranging from $6.7 \%$ to $38.2 \%$ of samples tested. The two Pf-pLDH RDTs did not show any prozone positive sample. Table 3 lists for each RDT brand and samples with high parasitemia the frequencies for which prozone was observed, matched with the line intensity of the undiluted sample. Among 104 test results with prozone, negative, faint and weak HRP-2 test lines were observed in $4(3.8 \%), 15(14.4 \%)$ and $85(81.7 \%)$ results. 
Two RDT brands accounted for all negative and faint test lines. The three samples with negative test lines (including one sample with negative results for two RDT brands) had parasite densities of $8.3 \%, 8.3 \%$ and $8.4 \%$ (Figure 2).

Table 3 Number of $P$. falciparum samples with parasite density $\geq 4 \%$ generating prozone for HRP-2 $(\mathrm{n}=6)$ and Pf- $\mathrm{pLDH}(\mathrm{n}=2)$ RDT brands.

\begin{tabular}{|c|c|c|c|c|c|c|}
\hline \multirow{2}{*}{$\begin{array}{c}\text { Brands / } \\
\text { manufacturers }\end{array}$} & \multirow{2}{*}{$\begin{array}{c}P . \\
\text { falciparum } \\
\text { antigen } \\
\text { targeted }\end{array}$} & \multirow{2}{*}{$\begin{array}{l}\text { Number } \\
\text { of } \\
\text { samples } \\
\text { tested }\end{array}$} & \multirow{2}{*}{$\begin{array}{c}\text { Total } \\
\text { number of } \\
\text { samples } \\
\text { with } \\
\text { prozone } \\
(\%)\end{array}$} & \multicolumn{3}{|c|}{$\begin{array}{l}\text { HRP-2 or Pf-pLDH line intensity for } \\
\text { undiluted prozone positive samples }\end{array}$} \\
\hline & & & & Negative & Faint & Weak \\
\hline $\begin{array}{l}\text { Paracheck- } \\
\text { Pf }\end{array}$ & HRP-2 & 76 & $29(38.2)$ & 3 & 5 & 21 \\
\hline ICT Malaria & HRP-2 & 76 & $27(35.5)$ & 1 & 10 & 16 \\
\hline $\begin{array}{l}\text { SD Malaria } \\
\text { Antigen Pf } \\
\text { FK50 }\end{array}$ & HRP-2 & 76 & $25(32.9)$ & - & - & 25 \\
\hline $\begin{array}{l}\text { Hexagon } \\
\text { Malaria } \\
\text { Combi }\end{array}$ & HRP-2 & 72 & 12 (16.7) & - & - & 12 \\
\hline $\begin{array}{l}\text { SD Malaria } \\
\text { Ag Pf/Pan } \\
\text { FK60 }\end{array}$ & HRP-2 & 76 & $6(7.9)$ & - & - & 6 \\
\hline $\begin{array}{l}\text { Malaria } \\
\text { Pan/Pv/Pf } \\
\text { Rapid Device }\end{array}$ & HRP-2 & 75 & $5(6.7)$ & - & & 5 \\
\hline $\begin{array}{l}\text { SD Malaria } \\
\text { pLDH FK } 40\end{array}$ & Pf-pLDH & 76 & - & - & - & - \\
\hline $\begin{array}{l}\text { CareStart } \\
\text { Malaria } \\
\text { pLDH }\end{array}$ & Pf-pLDH & 76 & - & - & - & - \\
\hline
\end{tabular}




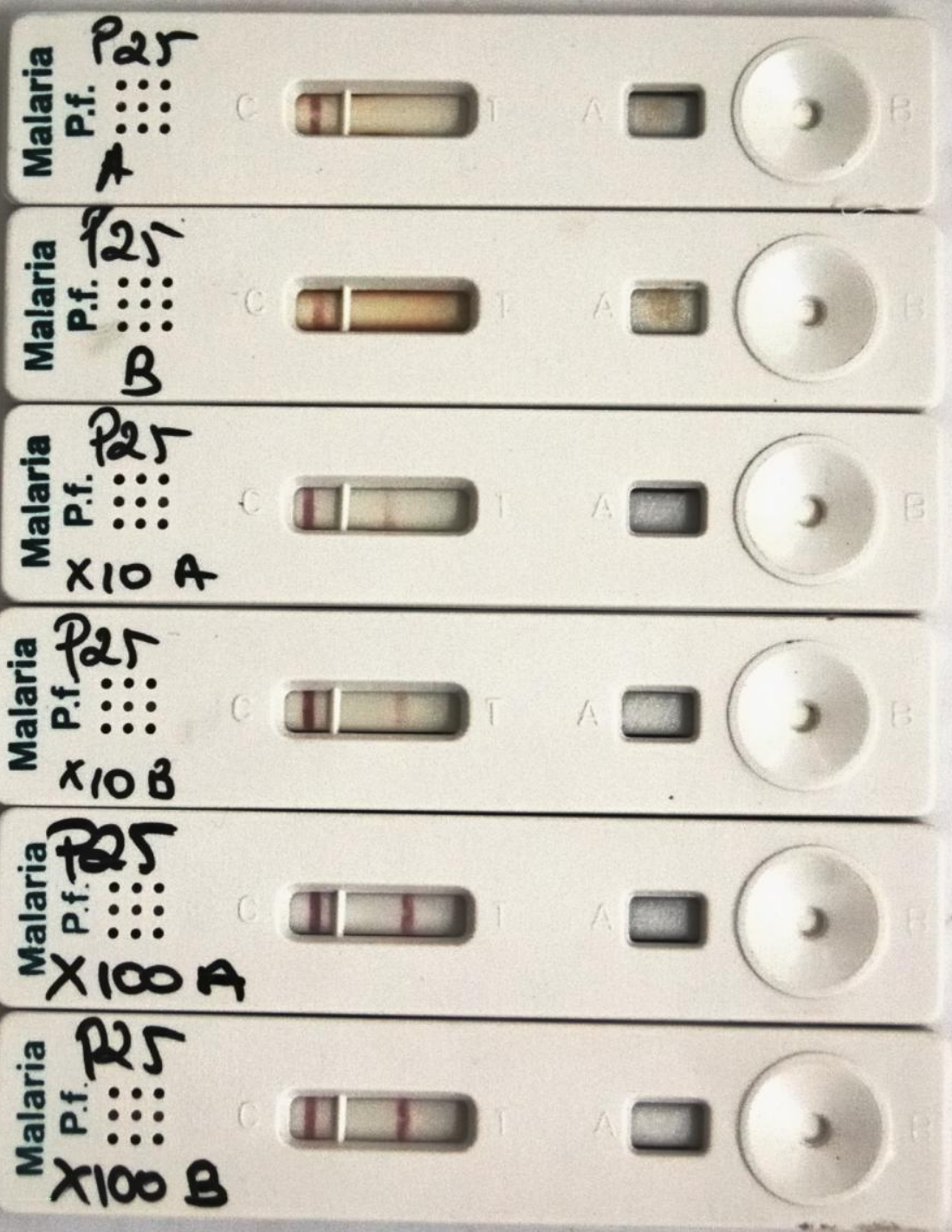

Figure 2: Example of prozone for Paracheck-Pf. Paracheck-Pf RDT cassettes run with a blood sample infected with $P$. falciparum at a parasite density of $8.3 \%$. The sample was assessed in duplicate, undiluted $(\mathrm{P} 25 \mathrm{~A}$ and $\mathrm{P} 25 \mathrm{~B}), 10 \times$ diluted $(\mathrm{P} 25 \times 10 \mathrm{~A}$ and $\mathrm{P} 25 \times 10 \mathrm{~B})$ and $100 \times$ diluted $(\mathrm{P} 25 \times 100 \mathrm{~A}$ and $\mathrm{P} 25$ $\times 100 \mathrm{~B})$. All cassettes show regular control lines, cassettes P25 A and B show no visible test line. Cassettes $\mathrm{P} 25 \times 10 \mathrm{~A}$ and $\mathrm{P} 25 \times 10 \mathrm{~B}$ show weak test lines and the maximum line intensity (strong) was obtained after $100 \times$ dilution (cassettes $\mathrm{P} 25 \times 100 \mathrm{~A}$ and $\mathrm{P} 25 \times 100 \mathrm{~B}$ ). 
Table 4 gives a breakdown of the proportions of prozone for the most affected (in terms of line intensities) RDT brand (Paracheck-Pf) according to different denominators. As an example, the proportions of false negative or faint HRP-2 lines were $10.9 \%, 1.2 \%$ and $0.1 \%$ among the samples with parasite densities $\geq 4 \%$, all $P$. falciparum positive samples and all suspected samples respectively. For the ICT malaria similar proportions were $14.1 \%$ (CI: 7.74-22.95); $1.5 \%$ (CI: $0.80-2.53$ ) and $0.2 \%$ (CI: 0.09-0.29) respectively.

Table 4: Proportion (\%) of prozone for Paracheck-Pf according to different denominators, $95 \%$ binomial confidence intervals $(\mathrm{CI})$ within brackets.

\begin{tabular}{|c|c|c|c|c|}
\hline Category & Number & $\begin{array}{c}\% \text { of patients } \\
\text { suspected of } \\
\text { malaria }\end{array}$ & $\begin{array}{c}\% \text { of } P . \\
\text { falciparum } \\
\text { positive patients }\end{array}$ & $\begin{array}{c}\% \text { of samples } \\
\text { with high } \\
\text { parasitemia }\end{array}$ \\
\hline $\begin{array}{l}\text { Patients suspected of } \\
\text { malaria ( } 1 \text { sample per } \\
\text { patient included })\end{array}$ & 7,543 & 100 & & \\
\hline $\begin{array}{l}\text { Samples positive for } \\
P \text {. falciparum }\end{array}$ & 873 & $\begin{array}{c}11.6 \\
(10.86-12.32)\end{array}$ & 100 & \\
\hline $\begin{array}{l}\text { Samples scored as } 4+ \\
\text { or } 5+(\mathrm{MoH} \text { semi- } \\
\text { quantitative scale })\end{array}$ & 410 & $\begin{array}{c}5.4 \\
(4.93-5.97)\end{array}$ & $\begin{array}{c}47.0 \\
(43.61-50.34)\end{array}$ & \\
\hline $\begin{array}{l}\text { Samples with a high } \\
\text { parasitemia (parasite } \\
\text { density } \geq 4 \% \text { ) }\end{array}$ & 92 & $\begin{array}{c}1.2 \\
(0.98-14.9)\end{array}$ & $\begin{array}{c}10,5 \\
(8,58-12,77)\end{array}$ & 100 \\
\hline $\begin{array}{l}\text { Number of samples } \\
\text { available for prozone } \\
\text { testing }\end{array}$ & 76 & - & - & - \\
\hline $\begin{array}{l}\text { Negative, faint or } \\
\text { weak HRP-2 test } \\
\text { lines }{ }^{*}\end{array}$ & 29 & $\begin{array}{c}0.5^{\dagger} \\
(0.32-0.64)\end{array}$ & $\begin{array}{c}4.0^{\dagger} \\
(2.81-5.53)\end{array}$ & $\begin{array}{c}38.0^{\dagger} \\
(28.12-48.76)\end{array}$ \\
\hline $\begin{array}{l}\text { Negative or faint } \\
\text { HRP-2 test lines }\end{array}$ & 8 & $\begin{array}{c}0.1^{\dagger} \\
(0.06-0.24)\end{array}$ & $\begin{array}{c}1.2^{\dagger} \\
(0.55-2.10)\end{array}$ & $\begin{array}{c}10.9^{\dagger} \\
(5.34-19.08)\end{array}$ \\
\hline $\begin{array}{l}\text { Negative HRP-2 test } \\
\text { lines }\end{array}$ & 3 & $\begin{array}{c}0.05^{\dagger} \\
(0.01-0.14)\end{array}$ & $\begin{array}{c}0.5^{\dagger} \\
(0.12-1.17)\end{array}$ & $\begin{array}{c}4.4^{\dagger} \\
(1.20-10.76)\end{array}$ \\
\hline
\end{tabular}

\footnotetext{
Line intensity for undiluted samples

${ }^{\dagger}$ Proportions calculated according to Table 2, corrected for the number of samples that were not available for prozone testing $(n=16)$.
}

Table 5 lists the clinical and laboratory data for the samples with and without prozone for Paracheck-Pf. Among the high parasitemia samples, prozone occurred most frequently but not exclusively among the group minus 5 years of age. 
Table 5: Characteristics of P. falciparum samples that were prozone positive or negative for ParacheckPf

Samples with high parasitemia (parasite density $\geq 4 \%$ )

\begin{tabular}{|c|c|c|c|}
\hline $\begin{array}{l}\text { Prozone } \\
\text { positive }\end{array}$ & $\begin{array}{l}\text { Prozone } \\
\text { negative }\end{array}$ & Total & $\mathrm{p}={ }^{*}$ \\
\hline $\mathrm{n}=29$ & $\mathrm{n}=47$ & $\mathrm{n}=76$ & \\
\hline
\end{tabular}

Demographic and clinical data (expressed as \% of total)

\begin{tabular}{|c|c|c|c|c|c|}
\hline \multirow[t]{3}{*}{ Patients $(n=76)$} & $\begin{array}{l}\text { Children }<5 \text { years } \\
(\mathrm{n}=53)\end{array}$ & 82.8 & 61.7 & 69.8 & NS \\
\hline & $\begin{array}{l}\text { Children } \geq 5 \text { years } \\
(\mathrm{n}=14)\end{array}$ & 10.3 & 23.4 & 18.4 & NS \\
\hline & $\begin{array}{l}\text { Adults (>14 years }) \\
(\mathrm{n}=9)\end{array}$ & 6.9 & 14.9 & 11.8 & NS \\
\hline $\begin{array}{l}\text { Gender ratio } \\
(\mathrm{n}=74)\end{array}$ & Male/female & 40.7 & 57.4 & 51.4 & NS \\
\hline \multirow[t]{4}{*}{$\begin{array}{l}\text { Presenting } \\
\text { symptoms }\end{array}$} & Fever $(n=64)$ & 96.3 & 97.3 & 96.9 & NS \\
\hline & Cough $(\mathrm{n}=59)$ & 50.0 & 11.4 & 27.1 & 0.002 \\
\hline & Vomiting $(\mathrm{n}=60)$ & 28.0 & 45.7 & 38.3 & NS \\
\hline & Diarrhoea $(\mathrm{n}=60)$ & 28.0 & 45.7 & 30.0 & NS \\
\hline $\begin{array}{l}\text { Clinical signs of } \\
\text { severity }\end{array}$ & $\begin{array}{l}\text { At least one } \\
(\mathrm{n}=60)\end{array}$ & 64.0 & 48.6 & 55.0 & NS \\
\hline $\begin{array}{l}\text { Laboratory sign } \\
\text { of severity }\end{array}$ & $\begin{array}{l}\text { Hemoglobin < } \\
\text { g/dl }(n=76)\end{array}$ & 20.7 & 6.4 & 11.8 & NS \\
\hline Signs of severity & $\begin{array}{l}\text { At least one } \\
\text { clinical or } \\
\text { laboratory }(\mathrm{n}=61)\end{array}$ & 69.2 & 54.3 & 60.7 & NS \\
\hline \multicolumn{6}{|c|}{ Laboratory values (expressed as median and $95 \% \mathrm{CI}$ ) } \\
\hline \multirow{2}{*}{$\begin{array}{l}\text { Parasite density } \\
\text { in } \%(n=76)\end{array}$} & Median & 8.0 & 6.9 & 6.9 & NS \\
\hline & Range & $4.0-28.7$ & $4.2-22.3$ & $4.0-28.7$ & \\
\hline \multirow{2}{*}{$\begin{array}{l}\text { Hemoglobin } \\
\text { level }(\mathrm{g} / \mathrm{dl}) \\
(\mathrm{n}=76)\end{array}$} & Median & 7.2 & 9.0 & 8.3 & 0.0074 \\
\hline & Range & $2.9-12.5$ & $2.5-13.3$ & $2.5-13.3$ & \\
\hline \multirow[t]{2}{*}{$\begin{array}{l}\text { RBC count } \\
\left(\times 10^{9} / 1\right)(n=76)\end{array}$} & Median & 3.1 & 3.8 & 3.4 & 0.0131 \\
\hline & Range & $1.1-5.3$ & $0.9-5.3$ & $0.9-5.3$ & \\
\hline \multirow[t]{2}{*}{$\begin{array}{l}\text { Platelet count } \\
\left(\times 10^{6} / 1\right)(\mathrm{n}=76)\end{array}$} & Median & 74 & 90 & 86 & NS \\
\hline & Range & $12-488$ & $13-390$ & $12-390$ & \\
\hline \multirow[t]{2}{*}{$\begin{array}{l}\text { WBC count } \\
\left(\times 10^{6} / 1\right)(n=76)\end{array}$} & Median & 10.4 & 10.1 & 10.2 & NS \\
\hline & Range & 4.4-19.9 & $3.2-27.6$ & $3.2-27.6$ & \\
\hline
\end{tabular}

${ }^{*} \mathrm{NS}=$ not significant 
No significant relation between prozone and the presenting symptoms was found. However, cough was significantly more prevalent among the prozone positive group. Of note, clinical signs of severity were not associated with prozone, and, moreover, prozone occurred also in $10(36 \%)$ patients without any sign of severe malaria. Among the laboratory values, the prozone group was significantly associated with lower hemoglobin levels and RBC counts. Parasite densities of prozone positive and prozone negative samples did not differ significantly.

Table 6 lists the number of HRP-2 based brands affected by prozone in relation to parasite density as well as the results for the subset of 45 additional samples of parasite densities < $4 \%$ (mean \pm SD parasite density: $2.7 \pm 0.9 \%$ ). Prozone occurred less frequently in samples with parasite densities $<4 \%$ compared to samples with high parasitemia $(13 / 45$ versus $39 / 76, p=0.016)$. Prozone occurred dispersedly among the samples but the intensity of prozone (in terms of numbers of negative, faint or weak test lines among undiluted samples) increased with parasite density ( $\mathrm{p}$ $=0.015)$. The three samples with prozone in five out of six HRP-2 RDTs had parasite densities of $8.7 \%, 11.5 \%$ and $28.7 \%$ respectively. Among the samples with parasite densities $<4 \%$, samples with negative test lines were not observed and prozone with faint test lines was observed for only one brand (ICT Malaria, at parasite densities of $2.2 \%, 2.9 \%$ and $3.4 \%$ respectively).

Table 7 lists the results for the additional HRP-2 RDT brands assessed with samples that were prozone positive for at least one of the brands assessed prospectively. These results confirmed the susceptibility of the HRP-2 RDT brands to prozone: prozone affected all four brands in proportions ranging from $20.6 \%$ to $85.0 \%$. Although no negative test results were obtained, faint test lines were observed in proportions up to $17.5 \%$. In addition, there was a clear difference between the two lot numbers of the Malaria Pan/Pv/Pf Rapid Device: for 43 samples assessed by both lots, prozone was observed in $5(11.6 \%)$ versus 22 (51.2\%) samples respectively $(\mathrm{p}<0.0005)$. 
Table 6: Number of HRP-2 RDT brands affected by prozone in relation to the parasite density and HRP-2 test line intensity for undiluted samples (negative, faint and weak).

\begin{tabular}{|c|c|c|c|c|c|c|c|c|c|c|c|}
\hline \multirow{2}{*}{$\begin{array}{c}\text { Parasite } \\
\text { density } \\
\%\end{array}$} & \multirow{2}{*}{$\begin{array}{l}\text { Number } \\
\text { of } \\
\text { samples } \\
\text { assessed }\end{array}$} & \multicolumn{2}{|c|}{$\begin{array}{c}\text { Prozone for at least } 1 \\
\text { RDTs }\end{array}$} & \multicolumn{2}{|c|}{$\begin{array}{c}\text { Prozone for at least } 2 \\
\text { RDTs }\end{array}$} & \multicolumn{2}{|c|}{$\begin{array}{c}\text { Prozone for at least } 3 \\
\text { RDTs }\end{array}$} & \multicolumn{2}{|c|}{$\begin{array}{c}\text { Prozone for at least } 4 \\
\text { RDTs }\end{array}$} & \multicolumn{2}{|c|}{$\begin{array}{c}\text { Prozone for at least } 5 \\
\text { RDTs }\end{array}$} \\
\hline & & Weak & $\begin{array}{l}\text { Faint } \\
\text { or no } \\
\text { line }\end{array}$ & Weak & $\begin{array}{c}\text { Faint } \\
\text { or no } \\
\text { line }\end{array}$ & Weak & $\begin{array}{l}\text { Faint } \\
\text { or no } \\
\text { line }\end{array}$ & Weak & $\begin{array}{l}\text { Faint } \\
\text { or no } \\
\text { line }\end{array}$ & Weak & $\begin{array}{c}\text { Faint } \\
\text { or no } \\
\text { line }\end{array}$ \\
\hline
\end{tabular}

\begin{tabular}{|c|c|c|c|c|c|c|c|c|c|c|c|}
\hline 1 to 3.9 & 45 & 10 & $3^{*}$ & 8 & - & 5 & - & 2 & - & - & - \\
\hline 4 to 4.9 & 18 & 4 & $4^{\dagger}$ & 8 & 0 & 7 & - & 2 & - & - & - \\
\hline 5 to 9.9 & 39 & 13 & $8^{\ddagger}$ & 11 & $2^{f}$ & 8 & - & 4 & - & 1 & - \\
\hline$\geq 10$ & 19 & 6 & $4^{\$}$ & 8 & $1^{+}$ & 8 & - & 3 & - & 2 & - \\
\hline
\end{tabular}

\footnotetext{
Parasite densities of 2.2, 2.9 and $3.4 \%$

$\dagger$ Parasite densities of 4.1, 4.2, 4.3 and $4.7 \%$

Parasite densities of 8.0, 8.0, 8.3, 8.3, 8.4, 8.6, 8.9 and $9.3 \%$

$£$ Parasite densities of 8.0 and $8.3 \%$

$\$$ Parasite densities of 10.6, 11.0, 11.5 and $28.7 \%$

+ Parasite densities of $28.7 \%$
} 
Table 7: Number of prozone positive samples for additional HRP-2 RDT brands that were assessed with samples positive for prozone with at least one of the RDTs from Table 3.

\begin{tabular}{|c|c|c|c|c|c|c|}
\hline \multirow[t]{2}{*}{ Brands / Manufacturers } & \multirow{2}{*}{$\begin{array}{l}P . \text { falciparum } \\
\text { antigen target }\end{array}$} & \multirow{2}{*}{$\begin{array}{c}\text { Number of } \\
\text { samples } \\
\text { tested }\end{array}$} & \multirow{2}{*}{$\begin{array}{c}\text { Total number } \\
\text { of samples } \\
\text { with prozone } \\
(\%)\end{array}$} & \multicolumn{3}{|c|}{$\begin{array}{l}\text { HRP-2 or Pf-pLDH line intensity for } \\
\text { undiluted prozone positive samples }\end{array}$} \\
\hline & & & & Negative & Faint & Weak \\
\hline Hexagon Malaria & HRP-2 & 39 & $13(33.3 \%)$ & - & 0 & 13 \\
\hline Malaria Total Quick & HRP-2 & 44 & $22(50.0 \%)$ & - & 5 & 17 \\
\hline First Response Malaria Ag Combo & HRP-2 & 40 & $34(85.0 \%)$ & - & 7 & 27 \\
\hline ICT Malaria Combo & HRP-2 & 34 & $7(20.6 \%)$ & - & 1 & 6 \\
\hline Malaria Pan/Pv/Pf Rapid ${ }^{\ddagger}$ Device & HRP-2 & 43 & $22(51.2 \%)$ & - & 4 & 18 \\
\hline
\end{tabular}




\section{Discussion}

In 2009, an estimated 225 million cases of malaria occurred with 781.000 deaths, mostly due to $P$. falciparum in children in Africa [1]. WHO recommends parasitological diagnostic testing before treatment. When microscopy is not available, RDTs are the alternative. RDTs have been demonstrated to perform equally well or even better than microscopy in field settings [20-23] and are currently deployed at all levels of health facilities [1].

Some limitations are however to be mentioned. The wet season of 2009-2010 in Mozambique was characterized by irregular rainfall and long dry spells. Due to this low rainfall (30\% below expected value in November 2009 up to $60 \%$ in March 2010 [24]) and the impact of local control measures in the months preceding the study, there was less malaria than expected and the originally planned $5 \%$ parasite density threshold (defined by WHO as hyperparasitemia [13]) was replaced by $4 \%$, also used in other studies $[14,15]$. Although samples below this $4 \%$ threshold were included, prozone was not assessed below this threshold, precluding systematic study of possible clinical or laboratory predictors of prozone. Further, due to nonavailability of trained staff around the clock, it was not always possible to record all clinical data and to work-up all samples in the laboratory. Likewise, for logistic reasons, two RDTs were assessed with blood samples stored at $-20^{\circ} \mathrm{C}$ and not on fresh samples. However, it should be noted that the HRP-2 antigen is very stable and resistant to harsh conditions [25]. For three RDTs, storage temperatures slightly exceeded those recommended by the manufacturers. Finally only a minority of undiluted prozone positive samples showed no visible test line, whereas the remaining samples showed faint or weak test lines. However, disregarding faint or even weak test lines as negative is a common error among end-users in field settings [26-28].

There are only few original studies reporting on prozone in malaria RDTs [9,29]. Prozone may however explain for the rare but consistent reports of false negative HRP-2 based RDT results in samples with high parasite density: for instance, in a recent study of two RDTs in Sierra Leone, two false-negative samples were observed with the HRP-2 RDT but not with the Pf-pLDH RDT. The parasite densities of both samples were $288,000 / \mu 1$ and $580.000 / \mu 1$, corresponding to $5.7 \%$ and $11.6 \%$ parasite density respectively [30]. Interestingly, the HRP-2 test used in this study was Paracheck-Pf and the two samples accounted for $1.1 \%$ of all malaria positive samples which is in line with the present findings. Similar observations affecting Paracheck-Pf or other HRP-2 RDT brands have been reported from endemic as well as non-endemic settings [31-33]. 
In line with previous findings, the present study demonstrated prozone in HRP-2 but not in Pf-pLDH based RDTs [9]. Compared to HRP-2 based RDTs, pLDH based RDTs are ascribed lower sensitivity and lower heat-stability [23,34,35], but according to a recent field study and the second WHO/FIND RDT evaluation round, Pf-pLDH RDTs may perform equally well as HRP-2 RDTs [17,36]. The observation of lot-to-lot variations in one HRP-2 RDT brand illustrates that small differences in composition may influence the vulnerability to prozone. In that way, it should be noted that the present data only reflect those of the examined lot numbers and may therefore not be extrapolated to all RDT brands.

From the present results, it is clear that prozone occurred dispersedly among samples with high parasitemia. Prozone with non-visible test lines occurred exclusively in samples with parasite densities above $8 \%$ and the frequency and intensity of prozone decreased below the $4 \%$ threshold. However, the association parasite density presence of prozone among samples with parasite densities $\geq 4 \%$ was not straightforward. This may be ascribed to factors such as capillary sequestration of the parasites, variations in the antigen production during the cycle and strain differences [4,37]. Prozone remains a rare phenomenon and although the present study was not designed to trace risk factors of prozone, this study suggests that, apart from hemoglobin level, there are no clear indicators for prozone.

For the two most affected RDT brand (Paracheck-Pf and ICT Malaria), prozone with negative or faint HRP-2 test lines (the most dangerous situation) occurred in at least $1.2 \%$ of malaria-positive samples and $10.9 \%$ of samples with high parasitemia. At such frequencies, the diagnostic accuracy may be affected and the impact on predictive values will depend on the malaria-attributable fraction of fevers and the proportion of high parasite densities: both factors are related to transmission intensity and pre-existing immunity of the affected population [38]. For Africa, the $P$. falciparum prevalence rate in children aged two to ten years is actually estimated at $17 \%$ [39]. For the distribution of parasite densities, published data are scarce. Two recent studies conducted in children in areas of perennial transmission in Gabon and Sierra Leone reported median parasite densities of $13,860 / \mu 1(1,400-71,452)$ and $264,000(1-2,136,000)[22,30]$. When extrapolating for Mozambique, based on the $4,310,086$ suspected malaria cases reported in 2009 [1], a 17\% malaria attributable fraction [39] and a $10 \%$ proportion of high parasitemia, the annual numbers of negative or faint test lines were calculated as 7,694 (CI: 3,883-13,922) and 9,643 (CI: 4,543-15,387) with Paracheck-Pf and ICT Malaria (tested with the presently evaluated lots) respectively.

The risk related to false-negative RDT results due to low parasite densities is mitigated by diagnostic algorithms recommending to repeat testing after an 
unexpected negative RDT result [40-42]. However, such policy will not timely correct for false-negative results due to the prozone, as hyperparasitemia represents a life-threatening situation. In addition, there is a tendency to roll out RDTs to poorly resourced peripheral health care facilities where there are no further laboratory facilities to perform sample dilution or microscopy in order to correct for prozone $[1,20]$. Possible other measures to address prozone are training of the enduser to understand the problem and to assure interpretation of faint test lines as positive test results. Concerning RDT quality control at the level of national reference laboratory, samples with hyperparasitemia could be included but in view of the low prozone frequency and its scattered distribution among samples with hyperparasitemia, it is difficult to assess prozone on a pre-release basis. Postmarketing follow-up including incident reporting could provide further clues. Finally, depending on the distribution of parasite densities in a given population, susceptibility to prozone should be added as a major argument in the strategic choice between Pf-pLDH and HRP-2 RDTs.

In conclusion, prozone is a rare event but it occurs among widely used HRP-2 RDTs at frequencies that may diminish diagnostic accuracy of the affected RDTs.

\section{List of abbreviations}

Ag: Antigen; CI: confidence intervals; EDTA: Ethylene Diamine Tetra-acetic Acid; FIND: Foundation for Innovative New Diagnostics; HRP-2: histidine-rich protein-2; ITM: Institute of Tropical Medicine; $\mathrm{NaCl}$ : Sodium Chloride; $\mathrm{MoH}$ : Ministry of Health; P.: Plasmodium; Pf: Plasmodium falciparum; PHT: Provincial Hospital of Tete; pan-pLDH: pan species parasite lactate dehydrogenase; Pf-pLDH: Plasmodium falciparum-specific parasite lactate dehydrogenase; $\mathrm{pLDH}$ : parasite lactate dehydrogenase; RDT(s): Rapid Diagnostic Test(s); TBF(s): thick blood film(s); WBC: white blood cell; RBC: red blood cell ;WHO: World Health Organization.

\section{Acknowledgements}

The authors would like to thank all patients and their parents/guardians for their participation. The authors are also grateful to the medical, paramedical and laboratory staff of the Provincial Hospital of Tete for their clinical and laboratory assistance. 


\section{References}

1. World Health Organization: World malaria report 2010. [http://whqlibdoc.who.int/publications/2010/9789241564106 eng.pdf]

2. Forney JR, Magill AJ, Wongsrichanalai C, Sirichaisinthop J, Bautista CT, Heppner DG, Miller RS, Ockenhouse CF, Gubanov A, Shafer R, DeWitt CC, Quino-Ascurra HA, Kester KE, Kain $\mathrm{KC}$, Walsh DS, Ballou WR, Gasser RA, Jr.: Malaria rapid diagnostic devices: performance characteristics of the ParaSight $\mathbf{F}$ device determined in a multisite field study. $J$ Clin Microbiol 2001, 39: 2884-2890.

3. Marx A, Pewsner D, Egger M, Nuesch R, Bucher HC, Genton B, Hatz C, Juni P: Meta-analysis: accuracy of rapid tests for malaria in travelers returning from endemic areas. Ann Intern Med 2005, 142: 836-846.

4. Murray CK, Gasser RA, Jr., Magill AJ, Miller RS: Update on Rapid Diagnostic Testing for Malaria. Clin Microbiol Rev 2008, 21: 97-110.

5. Ohrt C, Obare P, Nanakorn A, Adhiambo C, Awuondo K, O'Meara WP, Remich S, Martin K, Cook E, Chretien JP, Lucas C, Osoga J, McEvoy P, Owaga ML, Odera JS, Ogutu B: Establishing a malaria diagnostics centre of excellence in Kisumu, Kenya. Malar J 2007, 6: 79.

6. Pieroni P, Mills CD, Ohrt C, Harrington MA, Kain KC: Comparison of the ParaSight ${ }^{\mathrm{TM}}$-F test and the ICT Malaria PfrM test with the polymerase chain reaction for the diagnosis of Plasmodium falciparum malaria in travellers. Trans $R$ Soc Trop Med Hyg 1998, 92: 166-169.

7. Butch AW: Dilution protocols for detection of hook effects/prozone phenomenon. Clin Chem 2000, 46: 1719-1721.

8. Heidelberger M, Kendall FE: A quantitative theory of the precipitin reaction: II. a study of an azoprotein-antibody system. $J$ Exp Med 1935, 62: 467-483.

9. Gillet P, Mori M, Van Esbroeck M, Van Den Ende J, Jacobs J: Assessment of the prozone effect in malaria rapid diagnostic tests. Malar J 2009, 8: 271.

10. Mabunda S, Casimiro S, Quinto L, Alonso P: A country-wide malaria survey in Mozambique. I. Plasmodium falciparum infection in children in different epidemiological settings. Malar $J$ 2008, 7: 216.

11. Mabunda S, Aponte JJ, Tiago A, Alonso P: A country-wide malaria survey in Mozambique. II. Malaria attributable proportion of fever and establishment of malaria case definition in children across different epidemiological settings. Malar J 2009, 8: 74.

12. Ministério da Saùde and Republica de Moçambique: Manual de formação para o manejo de casos de malària. 2009. [

13. World Health Organization: Guidelines for the treatment of malaria, second edition, 2010. [http://whqlibdoc.who.int/publications/2010/9789241547925_eng.pdf ]

14. Bruneel F, Hocqueloux L, Alberti C, Wolff M, Chevret S, Bedos JP, Durand R, Le Bras J, Regnier B, Vachon F: The clinical spectrum of severe imported falciparum malaria in the intensive care unit: report of 188 cases in adults. Am J Respir Crit Care Med 2003, 167: 684689.

15. Bruneel F, Tubach F, Corne P, Megarbane B, Mira JP, Peytel E, Camus C, Schortgen F, Azoulay E, Cohen Y, Georges H, Meybeck A, Hyvernat H, Trouillet JL, Frenoy E, Nicolet L, Roy C, Durand R, Le Bras J, Wolff M: Severe imported falciparum malaria: a cohort study in 400 critically ill adults. PLoS One 2010, 5: e13236.

16. World Health Organization: Malaria Rapid Diagnostic Test Performance; Results of WHO product testing of malaria RDTs: Round 1 (2008). 2009. [http://www.finddiagnostics.org/resource-centre/reports brochures/malaria-diagnostics-report2009.html ]

17. World Health Organization: Malaria Rapid Diagnostic Test Performance; Results of WHO product testing of malaria RDTs: Round 2 (2009). 2010. [http://www.finddiagnostics.org/resource-centre/reports brochures/malaria-diagnostic-testreport-round2.html ]

18. World Health Organization: List of known commercially-available antigen-detecting malaria RDTs with adequate evidence of good manufacturing practice. 2009. [http://www.wpro.who.int/internet/resources.ashx/RDT/docs/MD_table34+(1)_totallistofISO131 485criteria.pdf ] 
19. Van der Palen M, Gillet P, Bottieau E, Cnops L, Van Esbroeck M, Jacobs J: Test characteristics of two rapid antigen detection tests (SD FK50 and SD FK60) for the diagnosis of malaria in returned travellers. Malar $J$ 2009, 8: 90.

20. Batwala V, Magnussen P, Nuwaha F: Are rapid diagnostic tests more accurate in diagnosis of plasmodium falciparum malaria compared to microscopy at rural health centres? Malar $J$ 2010, 9: 349.

21. Bjorkman A, Martensson A: Risks and benefits of targeted malaria treatment based on rapid diagnostic test results. Clin Infect Dis 2010, 51: 512-514.

22. Mawili-Mboumba DP, Bouyou Akotet MK, Ngoungou EB, Kombila M: Evaluation of rapid diagnostic tests for malaria case management in Gabon. Diagn Microbiol Infect Dis 2010, 66: 162-168.

23. Ochola LB, Vounatsou P, Smith T, Mabaso ML, Newton CR: The reliability of diagnostic techniques in the diagnosis and management of malaria in the absence of a gold standard. Lancet Infect Dis 2006, 6: 582-588.

24. World Food Programme: TRMM precipitation data analysis. 2011. [http://www.reliefweb.int/rw/fullmaps_af.nsf/luFullMap/EF66C9AFC68A9DD08525774300644 F80/\$File/map.pdf]

25. Makler MT, Piper RC: Rapid malaria tests: where do we go after 20 years? Am J Trop Med Hyg 2009, 81: 921-926.

26. Harvey SA, Jennings L, Chinyama M, Masaninga F, Mulholland K, Bell DR: Improving community health worker use of malaria rapid diagnostic tests in Zambia: package instructions, job aid and job aid-plus-training. Malar J 2008, 7: 160.

27. Mayxay M, Newton PN, Yeung S, Pongvongsa T, Phompida S, Phetsouvanh R, White NJ: Short communication: An assessment of the use of malaria rapid tests by village health volunteers in rural Laos. Trop Med Int Health 2004, 9: 325-329.

28. Rennie W, Phetsouvanh R, Lupisan S, Vanisaveth V, Hongvanthong B, Phompida S, Alday P, Fulache M, Lumagui R, Jorgensen P, Bell D, Harvey S: Minimising human error in malaria rapid diagnosis: clarity of written instructions and health worker performance. Trans $R$ Soc Trop Med Hyg 2007, 101: 9-18.

29. Risch L, Bader M, Huber AR: [False negative quick malaria test]. Schweiz Med Wochenschr 1999, 129: 1002.

30. Gerstl S, Dunkley S, Mukhtar A, De Smet M, Baker S, Maikere J: Assessment of two malaria rapid diagnostic tests in children under five years of age, with follow-up of false-positive pLDH test results, in a hyperendemic falciparum malaria area, Sierra Leone. Malar $J$ 2010, 9: 28 .

31. Bisoffi Z, Sirima SB, Menten J, Pattaro C, Angheben A, Gobbi F, Tinto H, Lodesani C, Neya B, Gobbo M, Van Den Ende J: Accuracy of a rapid diagnostic test on the diagnosis of malaria infection and of malaria-attributable fever during low and high transmission season in Burkina Faso. Malar J 2010, 9: 192.

32. Stow NW, Torrens JK, Walker J: An assessment of the accuracy of clinical diagnosis, local microscopy and a rapid immunochromatographic card test in comparison with expert microscopy in the diagnosis of malaria in rural Kenya. Trans $R$ Soc Trop Med Hyg 1999, 93: 519-520.

33. Wiese L, Bruun B, Baek L, Friis-Moller A, Gahrn-Hansen B, Hansen J, Heltberg O, Hojbjerg T, Hornstrup MK, Kvinesdal B, Gomme G, Kurtzhals JA: Bedside diagnosis of imported malaria using the Binax Now malaria antigen detection test. Scand J Infect Dis 2006, 38: 1063-1068.

34. Chiodini PL, Bowers K, Jorgensen P, Barnwell JW, Grady KK, Luchavez J, Moody AH, Cenizal A, Bell D: The heat stability of Plasmodium lactate dehydrogenase-based and histidine-rich protein 2-based malaria rapid diagnostic tests. Trans $R$ Soc Trop Med Hyg 2007, 101: 331337.

35. Drakeley C, Reyburn H: Out with the old, in with the new: the utility of rapid diagnostic tests for malaria diagnosis in Africa. Trans R Soc Trop Med Hyg 2009, 103: 333-337.

36. Fogg C, Twesigye R, Batwala V, Piola P, Nabasumba C, Kiguli J, Mutebi F, Hook C, Guillerm M, Moody A, Guthmann JP: Assessment of three new parasite lactate dehydrogenase (panpLDH) tests for diagnosis of uncomplicated malaria. Trans R Soc Trop Med Hyg 2008, 102: 25-31.

37. Bell D, Peeling RW: Evaluation of rapid diagnostic tests: malaria. Nat Rev Microbiol 2006, 4: S34-S38. 
38. Olliaro P: Management of fever and malaria - policy and practice. Trop Med Int Health 2009, 14: 488-490.

39. D'Acremont V, Lengeler C, Mshinda H, Mtasiwa D, Tanner M, Genton B: Time to move from presumptive malaria treatment to laboratory-confirmed diagnosis and treatment in African children with fever. PLoS Med 2009, 6: e252.

40. Farcas GA, Soeller R, Zhong K, Zahirieh A, Kain KC: Real-time polymerase chain reaction assay for the rapid detection and characterization of chloroquine-resistant Plasmodium falciparum malaria in returned travelers. Clin Infect Dis 2006, 42: 622-627.

41. Gillet P, Mukadi P, Vernelen K, Van Esbroeck M, Muyembe JJ, Bruggeman C, Jacobs J: External quality assessment on the use of malaria rapid diagnostic tests in a non-endemic setting. Malar J 2010, 9: 359.

42. Msellem MI, Martensson A, Rotllant G, Bhattarai A, Stromberg J, Kahigwa E, Garcia M, Petzold $\mathrm{M}$, Olumese $\mathrm{P}$, Ali A, Bjorkman A: Influence of rapid malaria diagnostic tests on treatment and health outcome in fever patients, Zanzibar: a crossover validation study. PLoS Med 2009, 6: e1000070.

43. World Health Organization: Information note on interim selection criteria for procurement of malaria rapid diagnostic tests (RDTs). 2010.

[http://www.who.int/entity/malaria/diagnosis_treatment/diagnosis/infoRDTinterimcriteria.pdf 


\section{Chapter IV}

\section{Buffer substitution in malaria rapid \\ diagnostic tests causes false-positive results}

Gillet P, Mori R, van den Ende J, Jacobs J.

Malaria Journal 2010; 9: 215.

http://www.malariajournal.com/content/9/1/215 
Buffer substitution in malaria rapid diagnostic tests causes false-positive results

\begin{abstract}
Background

Malaria rapid diagnostic tests (RDTs) are kits that generally include 20 to 25 test strips or cassettes, but only a single buffer vial. In field settings, laboratory staff occasionally uses saline, distilled water (liquids for parenteral drugs dilution) or tap water as substitutes for the RDT kit's buffer to compensate for the loss of a diluent bottle. The present study assessed the effect of buffer substitution on the RDT results.
\end{abstract}

\title{
Methods
}

Twenty-seven RDT brands were run with EDTA-blood samples of five malaria-free subjects, who were negative for rheumatoid factor and antinuclear antibodies. Saline, distilled water and tap water were used as substitute liquids. RDTs were also run with distilled water, without adding blood. Results were compared to those obtained with the RDT kit's buffer and Plasmodium positive samples.

\section{Results}

Only eight cassettes (in four RDT brands) showed no control line and were considered invalid. Visible test lines occurred for at least one malaria-free sample and one of the substitutes in 20/27 (74\%) RDT brands (saline: $\mathrm{n}=16$; distilled water: $\mathrm{n}=17$; and tap water: $\mathrm{n}=20$ ), and in 15 RDTs which were run with distilled water only. They occurred for all Plasmodium antigens and RDT formats (two-, three- and four-band RDTs). Clearance of the background of the strip was excellent except for saline. The aspects (colour, intensity and crispness) of the control and the false-positive test lines were similar to those obtained with the RDT kits' buffer and Plasmodium positive samples.

\section{Conclusion}

Replacement of the RDT kit's dedicated buffer by saline, distilled water and tap water can cause false-positive test results. 


\section{Background}

\section{The use of malaria RDTs is rapidly expanding}

Malaria rapid diagnostic tests (RDTs) detect Plasmodium parasites in blood by an antibody-antigen reaction on a nitrocellulose strip. Reactions are visible as cherryred lines. Two-band RDTs are mostly designed to detect Plasmodium falciparum; they display a control line and a test line, which targets either histidine-rich protein2 (HRP-2) or P. falciparum-specific parasite lactate dehydrogenase (Pf-pLDH). Three- and four-band RDTs display a control line and two or three test lines, one targeting $P$. falciparum specific antigen, another line targeting antigens common to the four species, such as pan-Plasmodium-specific lactate parasite dehydrogenase (pan-pLDH) or aldolase, and, in case of the four band RDTs, a third line which targets Plasmodium vivax-specific pLDH (Pv-pLDH).

RDTs are currently rolled out by national malaria control programmes in endemic settings as a tool for parasite based diagnosis in the scope of artemisinin-based combination therapy (ACT) [1]. In 2007, more than 70,000,000 tests were performed [2]. During the last few years, RDTs have technically improved and socalled one-step tests have replaced the older multistep tests. However, despite their apparent simplicity, they are not completely fail-proof $[3,4]$.

\section{The single vial of buffer in RDT kits may cause problems of availability}

Most malaria RDTs are marketed as kits that include material for 20 to 25 tests, such as lancets for finger pricking, test strips (available as dipstick, cassette or card box formats), transfer devices (pipettes, straws, capillaries or loops) and the buffer. Cassettes are usually individually packaged, and the number of lancets and transfer devices match the number of cassettes. All RDTs need a buffer to lyse the blood and to allow capillary flow along the nitrocellulose strip. Mostly, this buffer is supplied in a single bottle or dropper vial.

During field visits (particularly in Africa, ITM teams repeatedly observe availability problems of buffer vial: for instance, some cassettes were sent for testing in the ward, but the buffer vial did not return. To compensate, laboratory technicians took either a buffer vial from another kit (sometimes a kit of another brand), or used saline, distilled water (liquids for parenteral drugs dilution) and occasionally tap water as substitute liquids. Apparently, this substitution for the buffer seemed not to cause too much interference, as in all observed cases, there was enough background clearance and both control line and test lines were clearly distinguishable.

This phenomenon was also noted during a practical teaching session at the Institute of Tropical Medicine (ITM): students and staff were astonished to observe a visible 
HRP-2 line when the blood of one of the present authors (PG) was tested with a twoband RDT which was run with distilled water. PG had no symptoms of malaria, nor did he suffer from malaria in the recent past. When performing the RDT with the kit's dedicated buffer, there was no HRP-2 line visible. The HRP-2 line appeared upon retesting with distilled water and also when using saline and tap water as substitute liquids. As false-positive test line seemed to be the explanation, it was decided to explore this phenomenon.

\section{Methods}

\section{Samples of healthy subjects and Plasmodium positive samples}

EDTA-blood samples from five healthy subjects with no recent history of malaria were used. For all samples, the diagnosis of malaria was excluded by microscopy and species-specific PCR as previously described [5,6]. The presence of known causes of false positive RDT results such as the rheumatoid factor or antinuclear antibodies was ruled out [7-9] and none of the subjects had been manipulating mice during the past ten years, thereby reducing the probability false positive results due to anti-mouse antibodies [10]. For most experiments, fresh samples were used; samples stored at $-70^{\circ} \mathrm{C}$ were used in the case of delays of delivering of the RDTs. For comparison, all RDTs were run with their kit's dedicated buffer and two clinical samples, one infected with $P$. falciparum and another with $P$. vivax, at parasite densities of 36,140 and 3,600/ $\mu 1$ respectively.

\section{Choice of malaria RDTs}

Malaria RDTs marketed as cassettes and folded card box were selected, and RDT brands commonly used in field settings were included. CE marking and FDA approval of the RDTs were recorded, as well as their presence on the World Health Organization (WHO) lists of RDT manufacturers and distributors complying with ISO13485:2003 or US FDA 21 CFR 820 production norms [11] and their evaluation by the World Health Organization/Foundation for Innovative New Diagnostics (WHO/FIND) [12,13]. RDTs that were not on the local (Belgian) market were directly ordered from the manufacturer. In view of the wide lot-to-lot variations and the ever changing composition of RDTs, it was decided not to display the individual RDT brand and kit names, in line with previous comparative studies assessing characteristics of RDTs [14,15].

\section{RDT test procedures}

All RDT kits were used within their expiry date and had been stored at room temperature (maximum $25^{\circ} \mathrm{C}$ ) before analysis. RDTs were assessed in the same run with the five subjects' blood and the following buffers: RDT kit's dedicated buffer, distilled water (Denolin, Brussels, Belgium), saline ( $\mathrm{NaCl} 0.9 \%$, Qualiphar, Bornem 
Belgium) and tap water from local supply. For each RDT and substitute liquid, the blood samples of all five subjects were used except when a particular RDT kit was finished. In addition, tests were run in the absence of blood, with distilled water as the substitute liquid.

All tests were performed according to the instructions of the manufacturer, except that samples were loaded with a pipette (Finnpipette, Helsinki, Finland) instead of the transfer device supplied by the manufacturer. Readings were performed by three readers at daylight assisted by a standard electric bulb, and within and not beyond the prescribed delay after application of the sample and buffer.

\section{Interpretation of results}

In case the control line did not appear, the result was considered as invalid and the test was repeated. RDT test lines were interpreted according to the manufacturers' instructions. In addition, test line intensities were scored into five categories: none (no line visible), faint (barely visible line), weak (paler than the control line), medium (equal to the control line) or strong (stronger than the control line) [6]. Observers were blinded to each others' reading. The results of the readings considered were based on consensus agreement [16].

The appearance, shape and crispness of the control and test lines and the clearance of the background were compared with those obtained with the RDT kit's dedicated buffer and the two Plasmodium-positive samples. Visible test lines observed in the malaria free subjects' samples will be further referred to as "false-positive test lines".

\section{Inter-observer agreement and reproducibility}

Inter-reader reliability was assessed and expressed as percentage agreements for all three readers and kappa values for each pair of readers. To assess test reproducibility, a sample from one subject was tested upon five occasions for all RDT brands and the three substitute liquids. Two RDT brands were not included in the reproducibility assessment because of shortage of tests.

\section{Ethical review}

The study was approved by the Institutional Review Board of ITM and by the Ethical Committee of Antwerp University, Belgium. 


\section{Results}

\section{Selection of malaria rapid diagnostic kits}

Thirty different RDT brands were selected. As two brands from the same manufacturer showed bad clearance of the background upon testing with Plasmodium-positive samples and another brand had a very low specificity, they were not included in the study. The final panel consisted of 27 brands (26 cassette and 1 card box format). Eleven (40.7\%) of them had CE mark compliance, one was approved by the U.S. Food and Drug Administration, 22 (81.5\%) were included in the WHO list of RDTs adequate evidence of good manufacturing practice [11], 22 $(81.5 \%)$ were evaluated by the World Health Organization/Foundation for Innovative New Diagnostics [12,13], and 9 (30\%) are included in the list of malaria RDTs eligible for procurement by WHO [17]. The 27 RDT brands comprised two-, three- and four-band RDTs (Table 1) and all Plasmodium target antigens (HRP-2, Pf-pLDH, Pv-pLDH, pan-pLDH and aldolase) (Table 2).

Table 1: Numbers of RDT brands showing false positive test lines when run with blood of malaria free subjects and substitute liquids.

\begin{tabular}{|c|c|c|c|c|c|}
\hline \multirow{2}{*}{$\begin{array}{l}\text { RDT } \\
\text { format }\end{array}$} & \multirow{2}{*}{$\begin{array}{c}\text { Numbers of } \\
\text { different RDT } \\
\text { brands assessed }\end{array}$} & \multicolumn{4}{|c|}{$\begin{array}{c}\text { Numbers of RDTs brands showing false positive test lines when } \\
\text { run with substitute liquid* }\end{array}$} \\
\hline & & Saline & $\begin{array}{c}\text { Distilled } \\
\text { water }\end{array}$ & Tap water & $\begin{array}{l}\text { Any substitute } \\
\text { liquid }\end{array}$ \\
\hline Two band & 6 & $3(1)$ & $3(1)$ & $4(1)$ & $4(1)$ \\
\hline Three band & 17 & $12(9)$ & $11(10)$ & $12(9)$ & $12(10)$ \\
\hline Four band & 4 & $1(0)$ & $3(2)$ & $4(2)$ & $4(2)$ \\
\hline Total & 27 & $16(10)$ & 17 (13) & $20(12)$ & $20(13)$ \\
\hline
\end{tabular}

*Numbers refer to the different RDT brands for which false positive test lines were visible in at least one sample. Between brackets: numbers of RDT brands for which false positive lines were visible in the samples of at least three subjects.

\section{Results for the RDTs when run with samples of malaria free subjects and substitute liquids}

When run with their kit's buffer, none of the RDT brands tested positive with any of the samples of the five malaria free subjects. Likewise, when tested with the two Plasmodium positive blood samples and the RDT kit's buffer, the expected test line results were observed. 
When tested with a substitute liquid, there were eight invalid test results; they neither showed a control line upon repetition. Five of them occurred in a single RDT brand when assessed with distilled water. False-positive test lines were visible for at least one sample and substitute liquid in 20/27 (74\%) RDT brands, at the following frequencies: saline: $\mathrm{n}=16$, distilled water $\mathrm{n}=17$ and tap water: $\mathrm{n}=20$ (Table 1). They occurred randomly among the samples of the five malaria free subjects, and occurred in at least three of them. For 10 RDT brands, all five blood samples tested positive with at least one of the substitute liquids (saline: $\mathrm{n}=4$, distilled water: $\mathrm{n}=$ 5, tap water: $\mathrm{n}=7$ ). Two-, three- and four-band RDTs were all affected, as well as all Plasmodium target antigens (Table 2).

Table 2: Numbers of false-positive test lines for the different Plasmodium antigens of the RDTs when run with blood of malaria free subjects and substitute liquids.

\begin{tabular}{|c|c|c|c|c|c|}
\hline \multirow{2}{*}{$\begin{array}{l}\text { RDT target } \\
\text { antigen }\end{array}$} & \multirow{2}{*}{$\begin{array}{l}\text { Numbers of RDT } \\
\text { brands }(n=27) \\
\text { detecting the target }\end{array}$} & \multirow{2}{*}{$\begin{array}{l}\text { Total numbers of } \\
\text { tests performed with } \\
\text { each substitute* }\end{array}$} & \multicolumn{3}{|c|}{$\begin{array}{l}\text { Numbers of false positive test lines } \\
\text { when run with substitute liquid }\end{array}$} \\
\hline & & & Saline & $\begin{array}{l}\text { Distilled } \\
\text { water }\end{array}$ & Tap water \\
\hline HRP-2 & 21 & 98 & 22 & 28 & 36 \\
\hline Pf-pLDH & 5 & 25 & 8 & 9 & 9 \\
\hline Pv-pLDH & 9 & 45 & 12 & 21 & 20 \\
\hline Pan-pLDH & 14 & 70 & 30 & 25 & 33 \\
\hline Aldolase & 3 & 11 & 1 & 5 & 5 \\
\hline
\end{tabular}

"Each RDT brand was assessed with blood of five malaria-free subjects except one HRP-2-based kit and one HRP-2/aldolase-based kit that were assessed with two and one samples respectively.

For distilled water and tap water as the substitute liquid, the RDT strips with falsepositive lines showed a clearance of background similar to those observed with the RDT kit's buffer. In the case of saline, the background was less clear. Overall, the colour and crispness of the control and false-positive test lines were similar to those obtained with the RDT kits' buffers and Plasmodium positive samples (Figure 1 and 2). The proportions of medium and strong line intensities for all substitute liquids combined were as follows: $26.7 \%$ (23/86) for HRP-2, $11.5 \%$ (3/26) for Pf-pLDH, $37.7 \%$ (20/53) for Pv-pLDH and 29.6\% (26/88) for pan-pLDH. All false positive aldolase lines $(n=11)$ were either faint or weak. To the exception of better clearance of the background, the use of frozen sample did not influence the outcome of the results. 


\section{Results for the RDTs when run with distilled water only}

When tested with distilled water in the absence of blood, there was only a single invalid cassette, in which neither a control line appeared upon retesting. Control lines were observed in all remaining runs. Twenty-two false positive test lines were observed in $15(55.6 \%)$ RDT brands and occurred in all Plasmodium targets (Table 3). Control and false positive test lines showed colour, thickness and crispness similar to those obtained with the RDT kits' buffers and Plasmodium-positive samples (Figure 1 and 2). The line intensities of false positive has however tended to be lower (Table 3).

Table 3: Numbers of false-positive test lines for the different Plasmodium antigens of the RDTs when run with distilled water in absence of blood sample.

\begin{tabular}{lcccccc}
\hline \multirow{2}{*}{$\begin{array}{c}\text { RDT target } \\
\text { antigen }\end{array}$} & $\begin{array}{c}\text { Numbers of RDT } \\
\text { brands }(\mathrm{n}=27) \\
\text { detecting the } \\
\text { target }\end{array}$ & $\begin{array}{c}\text { Numbers of } \\
\text { false positive } \\
\text { test lines }\end{array}$ & \multicolumn{2}{c}{$\begin{array}{c}\text { Numbers of false positive test lines } \\
\text { according to test line intensity }\end{array}$} \\
\cline { 5 - 8 } HRP-2 & 21 & 11 & 2 & 5 & 3 & Faint \\
Pf-pLDH & 5 & 2 & 1 & 1 & 0 & 0 \\
Pv-pLDH & 9 & 1 & 1 & 0 & 0 & 0 \\
Pan-pLDH & 14 & 6 & 1 & 4 & 1 & 0 \\
Aldolase & 3 & 2 & 1 & 1 & 0 & 0 \\
\hline
\end{tabular}

\section{Inter-reader reliability and reproducibility}

The inter-observer agreement for positive and negative readings was high, with 96.4\% overall agreement between the three observers and kappa values between 0.94-0.98 for each pair out of three observers, Inter-observer agreements were similar for all three substitute liquids. Discrepancies between line intensity readings were limited to one category of line intensity (e.g., line intensity read as weak by reader one and as medium (but not strong) by reader two).

At reproducibility testing, false positive test lines occurred in at least four out of five runs in approximately half of the Plasmodium targets lines affected (40.0\% (10/25), $51.9 \%(14 / 27)$ and $51.6 \%(16 / 31)$ ) for saline, distilled water and tap water respectively). In terms of RDT brands, they occurred in at least four runs in eight, eleven and nine brands for saline, distilled water and tap water respectively.

\section{Differences between the different RDT brands}

CE-mark and non-CE labelled RDT brands were equally affected by the buffer substitution (11 out of 20, versus four out of seven). In addition, there were 11/22 WHO-GMP listed RTD brands affected versus four out of five RDTs that were not listed, 12/22 FIND evaluated brands versus three out of seven, and five out of nine RDTs from the WHO procurement list affected versus 10/18. Although the false- 
positive test lines occurred randomly among the different RDT brands, there were certain RDT brands that were more affected than others.

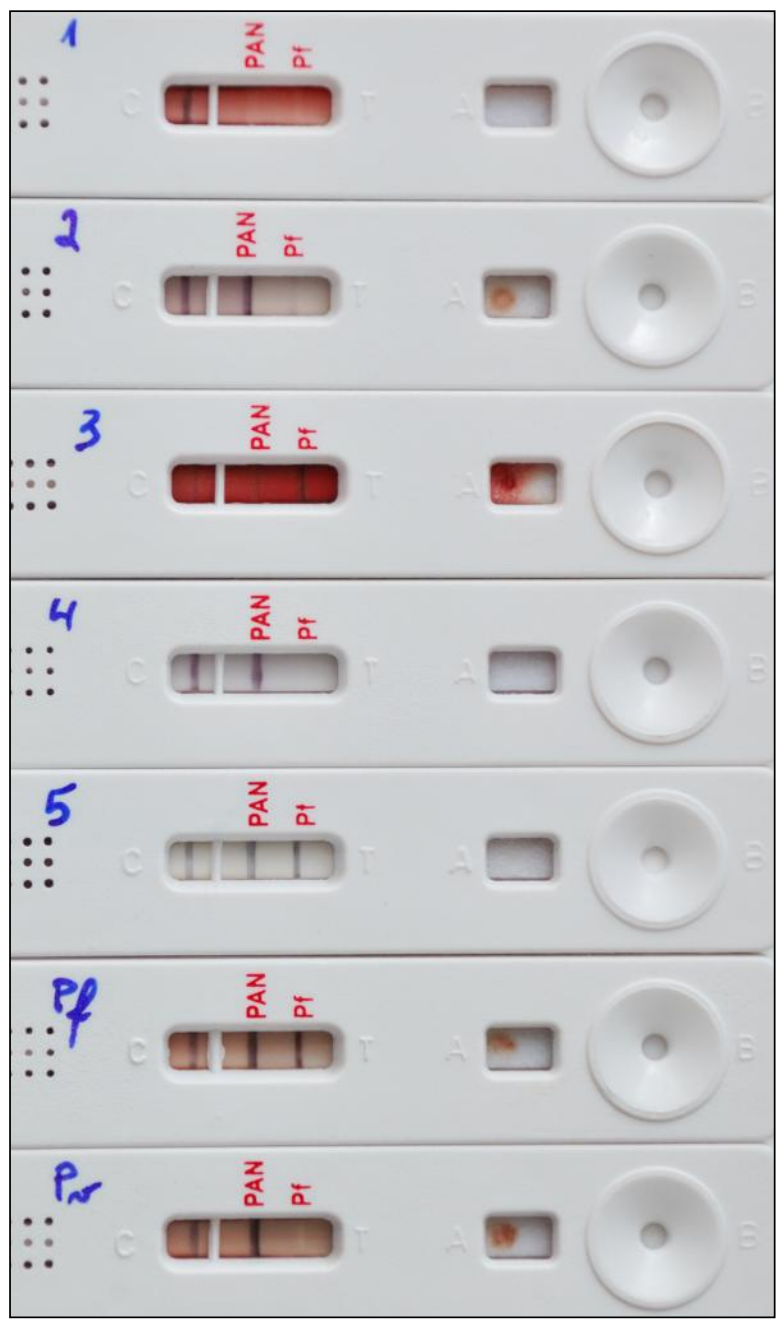

Figure 1: Example of buffer substitution's effect on a three-band RDT.

Three-band RDT cassettes run with blood of a malaria free subject and the RDT kit's dedicated buffer (1), injection water (2), saline (3), tap water (4) and when run with injection water in absence of blood sample (5). Cassettes "Pf" and "Pv" refer to Plasmodium falciparum or Plasmodium vivax positive samples run with the RDT kit's dedicated buffer. All cassettes show regular control lines, cassette 1 shows the expected result (no test line visible), cassettes 2, 3 and 5 show false positive pan-pLDH and Pf-HRP2lines, cassette 4 shows a false positive pan-pLDH line, and cassettes Pf and Pv show the expected results (positive for pan-pLDH and Pf-HRP-2 lines or positive for pan-pLDH line respectively). In the cassette 3 , the false positive reaction is partially masked by the background. 


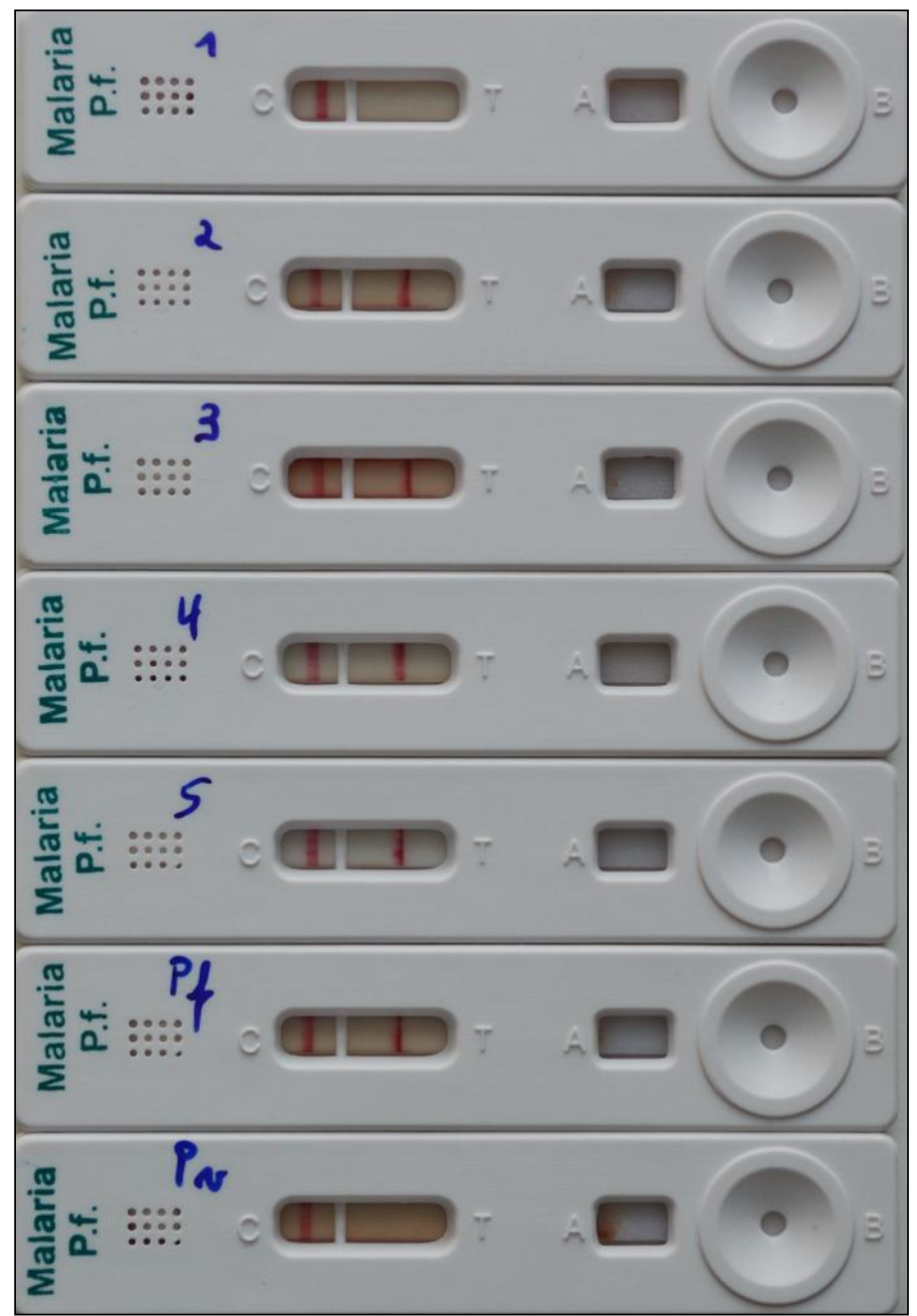

Figure 2: Example of buffer substitution's effect on a two-band RDT.

Cassettes of a two-band RDT run with blood of a malaria free subject and the RDT kit's dedicated buffer (1), injection water (2), saline (3), tap water (4) and when run with injection water in absence of blood sample (5). Cassettes "Pf" and "Pv" refer to Plasmodium falciparum or Plasmodium vivax positive samples run with the RDT kit's dedicated buffer. All cassettes show regular control lines, cassette 1 shows the expected result (no test line visible), cassettes 2, 3, 4 and 5 show false positive Pf-HRP-2 line, cassettes "Pf" and "Pv" show the expected results (positive for Pf-HRP-2 line or no test line visible respectively). 


\section{Discussion}

This study shows that replacing malaria RDT kit's buffers with saline, distilled water and tap water resulted in false-positive test lines in the majority of brands assessed. All Plasmodium targets and RDT formats were affected, and the control and false positive test lines observed were similar to those obtained with Plasmodium-positive samples and the RDT kits' buffers. In addition, approximately half of the RDT brands showed false positive test lines when run with distilled water in the absence of blood.

Malaria RDTs detect Plasmodium antigens by antibody-antigen interactions on a nitrocellulose test strip. The patient's blood and several drops of buffer are applied to the sample pad of the strip. They are attracted by the capillary action of a soak pad at the other end of the strip and start to migrate. First, they pass the so-called conjugation pad, which contains a detection antibody targeted to a Plasmodium antigen, such as HRP-2, Pf-pLDH, Pv-pLDH, pan-pLDH and aldolase. This detection antibody is a mouse-antibody that is conjugated to a signal, mostly colloidal gold. If present in the sample, the Plasmodium antigen is bound to this detection antibody-conjugate. The antigen-antibody-conjugate complex migrates further across the strip until it is bound to a second antibody, the so-called capture antibody. This capture antibody reacts to another epitope of the Plasmodium target antigen. As the capture antibody is applied on a narrow section of the strip, the complex with the conjugated signal will be concentrated and becomes visible as a cherry-red coloured line. The excess of detection antibody-conjugate that was not bound by the antigen and the capture antibody moves further towards the soak pad until it is bound to a goat anti-mouse antibody, thereby generating a control line $[10,18]$.

The present study has its limitations. First, there are currently more than 80 different RDT brands marketed [1], by consequence the results of the present panel of 27 brands should not be extrapolated to all RDTs. However, some out of these 80 brands are identical products marketed either as strips and cassette formats, of which the cassette form was tested in this study, given the fact that cassettes proved to be superior in terms of end-user performance [19-21]. In addition, representative RDT brands were elected with a majority of them included in the WHO list of RDTs adequate evidence of good manufacturing practice [11], evaluated by WHO/FIND $[12,13]$ or eligible for procurement by WHO [17]. Another limitation is the fact that the presence of anti-mouse antibodies, which can potentially produce false positive test lines was not ruled out by serologic testing [10]. However, none of the subjects reported long-term contact with mice, and the results obtained with the RDT kits' buffer were invariably negative. The most prevalent causes of false positive test 
lines from the sample side were excluded (rheumatoid factor and anti-nuclear antibodies) and the recommended reading delays were carefully respected thereby avoiding the backflow phenomenon: backflow is reverse migration of the antibodysignal conjugate with nonspecific bindings at the test line site, it is cited as the most common cause of false-positive reactions in RDTs [10]. Finally the physicochemical causes of the false-positive test lines were not explored, partly because of technical limitations, partly because buffer compositions are often proprietary.

The presence of the control line is the result of the binding of the conjugated mouseraised detection antibody to a goat-raised anti-mouse capture antibody: irrespective of the correct buffer there will be nearly always a control line visible as long as migration has been achieved. For the false-positive test lines, the situation is different. Their scattered distributions within the five malaria-free subjects and their moderate consistency upon retesting suggest nonspecific reactions, such as binding of the negatively charged colloidal gold conjugate to the positively charged capture antibodies. As the false-positive lines also occurred with distilled water in the absence of blood, a crucial role for the buffer is apparent. Apart from lysis of the red blood cells and allowing capillary migration of the sample along the strip, the buffer has other functions: it re-solubilizes blocking agents, such as detergents, polymers and proteins on the sample pad and the dried detection antibody-colloidal gold conjugate on the conjugate pad. Further, it ensures optimal $\mathrm{pH}$ and ionic strengths for the antigen-antibody reactions [10,18,22,23]. Substitution of the buffer may contribute to the non-specific bindings of the conjugate to the capture antibody in different ways: (i) less stringent $\mathrm{pH}$ and ionic strength conditions allowing nonspecific bindings, (ii) inefficient solubilisation of blocking agents from the sample pad and (iii) a slower capillary flow rate which in turn decreases flushing of the nonspecific bindings. Adding to this are mechanical issues: during application of the capture antibody, the dispensing pipette may emboss a groove in the membrane, with an additional decrease in capillary flow rate [22].

The high amount of false positive test lines with substitute liquids was unexpected and has, to the best of our knowledge, not yet been reported. The fact that the false positive test lines showed colour and crispness similar to those generated by Plasmodium-positive samples adds to the problem. In addition, their line intensities were comparable (albeit somewhat lower) to those observed in previous RDT evaluations at ITM [6,16,24-26] and among the affected RDT brands, there were two-band RDT brands that are currently widely deployed in endemic field settings.

Although the phenomenon of buffer substitution was observed in various places and on different occasions, the real extent of this phenomenon in the field is unknown. Studies assessing errors made by end-users do not mention it [21,27] but their 
designs (observations by checklists) were not adapted to assess incidental errors. It is tempting to speculate that at least some of the reported discrepancies between molecular tests and results from RDTs in field settings might be attributed to buffer substitution: as an example, Veron and Carme reported apparently false-positive RDT results for which they raised the possibility of incorrect performance [28].

False negative and false positive might delay/or exclude true diagnosis, with consequences that can go up to death for patients. The consequences of falsepositive RDTs extend those of individual patient care and the non-justified prescription of ACT treatment: like other errors by end-users, poor performance of RDTs will erode the health care workers' confidence in RDT test results thereby hindering the implementation of RDTs in treatment algorithms and malaria control programs [2]. Furthermore, as the buffer helps to ensure optimal conditions of $\mathrm{pH}$ and ionic strength, there is also a concern of possible false-negative results. Although this was not addressed in the present study, anecdotal information from colleagues and alumni in the field indicated also false negative results in case of simple substitution of the RDT kit's buffer by a vial from another kit, even from the same brand but from a different lot. Therefore minimal changes of buffer composition seem to cause critical effects. Finally, one might question whether other rapid diagnostic tests such as those detecting HIV-antibodies also suffer from this phenomenon.

Prevention of buffer substitution can be addressed in several ways. From daily use and evaluations of RDTs, it clear that some manufacturers already supply buffer vials with a volume in excess to the numbers of tests included. A solution could be the provision of more than one vial per kit, ideally (if costs are not too high), small plastic vials dedicated for each individual cassettes, , as is already the case for individually wrapped packages for self-testing (example: CareStart® Malaria, Single Kit, Access Bio Inc., New Jersey, USA).

Complementary, RDT package inserts should mention not to use any other liquids apart from the buffer supplied with the kit. Likewise, the generic job aids on malaria RDT designed by WHO [29] could include a comment on the use of the RDT kit's buffer.

The issue of buffer substitution should further be addressed in RDT instructions and trainings at all levels of health care organization. With respect to the organization of RDT performance by the end users among health care workers, it is recommended that use of the correct buffer should be supervised by a laboratory officer and that the RDT kits' content should not be split. 


\section{Conclusions}

In conclusion, buffer substitution in malaria RDTs causes false positive test lines in the majority of brands tested. Preventive measures in terms of product design, packaging, instructions manuals and trainings are needed to alert for this potential error.

\section{List of abbreviations}

ACT: Atimisinin based combination therapy; CE: Conformité Européenne; EDTA: Ethylene diamine tetra-acetic acid; FDA: Food and drug administration; FIND: Foundation for Innovative New Diagnostics; GMP: Good manufacturing practice; HRP-2: Histidine-rich protein 2; ISO: International organization for standardization; ITM: Institute of Tropical Medicine; NaCl: Sodium chloride; P: Plasmodium; PanpLDH: pan Plasmodium-specific parasite lactate dehydrogenase; PCR: Polymerase chain reaction; Pf-pLDH: Plasmodium falciparum-specific parasite lactate dehydrogenase; pLDH: parasite lactate dehydrogenase; Pv-pLDH: Plasmodium vivax-specific parasite lactate dehydrogenase; RDT(s): Rapid diagnostic test(s); WHO: World Health Organization. 


\section{References}

1. Drakeley $\mathrm{C}$, Reyburn $\mathrm{H}$ : Out with the old, in with the new: the utility of rapid diagnostic tests for malaria diagnosis in Africa. Trans R Soc Trop Med Hyg 2009, 103: 333-337.

2. Bell D, Perkins MD: Making malaria testing relevant: beyond test purchase. Trans $R$ Soc Trop Med Hyg 2008, 102: 1064-1066.

3. Murray CK, Gasser RA, Jr., Magill AJ, Miller RS: Update on Rapid Diagnostic Testing for Malaria. Clin Microbiol Rev 2008, 21: 97-110.

4. Wiese L, Bruun B, Baek L, Friis-Moller A, Gahrn-Hansen B, Hansen J, Heltberg O, Hojbjerg T, Hornstrup MK, Kvinesdal B, Gomme G, Kurtzhals JA: Bedside diagnosis of imported malaria using the Binax Now malaria antigen detection test. Scand J Infect Dis 2006, 38: 1063-1068.

5. Rougemont M, Van Saanen M, Sahli R, Hinrikson HP, Bille J, Jaton K: Detection of four Plasmodium species in blood from humans by $18 \mathrm{~S}$ rRA gene subunit-based and speciesspecific real-time PCR assays. J Clin Microbiol 2004, 42: 5636-5643.

6. Van der Palen M, Gillet P, Bottieau E, Cnops L, Van Esbroeck M, Jacobs J: Test characteristics of two rapid antigen detection tests (SD FK50 and SD FK60) for the diagnosis of malaria in returned travellers. Malar J 2009, 8: 90.

7. Grobusch MP, Jelinek T, Hanscheid T: False positivity of rapid antigen detection tests for diagnosis of Plasmodium falciparum malaria: issue appears to be more complicated than presented. J Clin Microbiol 1999, 37: 3781-3782.

8. Grobusch MP, Alpermann U, Schwenke S, Jelinek T, Warhurst DC: False-positive rapid tests for malaria in patients with rheumatoid factor. Lancet 1999, 353: 297.

9. Iqbal J, Sher A, Rab A: Plasmodium falciparum histidine-rich protein 2-based immunocapture diagnostic assay for malaria: cross-reactivity with rheumatoid factors. $J$ Clin Microbiol 2000, 38: 1184-1186.

10. Bell D, Peeling RW: Evaluation of rapid diagnostic tests: malaria. Nat Rev Microbiol 2006, 4: S34-S38.

11. World Health Organization: List of known commercially-available antigen-detecting malaria RDTs with adequate evidence of good manufacturing practice. 2009.

[http://www.wpro.who.int/NR/rdonlyres/E3597CBA-2ED4-4FCC-AB1CCE25FC6AA30E/0/M_D Webbuy Table9.pdf] Accessed 5 April 2009.

12. World Health Organization: Malaria Rapid Diagnostic Test Performance; Results of WHO product testing of malaria RDTs: Round 1 (2008). 2009.

[http://www.finddiagnostics.org/resource-centre/reports_brochures/malaria-diagnostics-report2009.html] Accessed 4 May 2010.

13. World Health Organization: Malaria Rapid Diagnostic Test Performance; Results of WHO product testing of malaria RDTs: Round 2 (2009). 2010.

[http://www.finddiagnostics.org/resource-centre/reports brochures/malaria-diagnostic-testreport-round2.html] Accessed 4 May 2010.

14. Chiodini PL, Bowers K, Jorgensen P, Barnwell JW, Grady KK, Luchavez J, Moody AH, Cenizal A, Bell D: The heat stability of Plasmodium lactate dehydrogenase-based and histidine-rich protein 2-based malaria rapid diagnostic tests. Trans $R$ Soc Trop Med Hyg 2007, 101: 331337.

15. Gillet P, Mori M, Van Esbroeck M, Van den Ende J, Jacobs J: Assessment of the prozone effect in malaria rapid diagnostic tests. Malar J 2009, 8: 271.

16. Gillet P, Bosselaers K, Cnops L, Bottieau E, Van Esbroeck M., Jacobs J: Evaluation of the SD FK70 malaria Ag Plasmodium vivax rapid diagnostic test in a non-endemic setting. Malar $J$ 2009, 8: 129.

17. World Health Organization: Information note on interim selection criteria for procurement of malaria rapid diagnostic tests (RDTs). 2010.

[http://www.who.int/entity/malaria/diagnosis treatment/diagnosis/infoRDTinterimcriteria.pdf] Accessed 4 May 2010.

18. Moody AH, Chiodini PL: Non-microscopic method for malaria diagnosis using OptiMAL IT, a second-generation dipstick for malaria pLDH antigen detection. Br J Biomed Sci 2002, 59: 228-231.

19. Bharti PK, Silawat N, Singh PP, Singh MP, Shukla M, Chand G, Dash AP, Singh N: The usefulness of a new rapid diagnostic test, the First Response Malaria Combo (pLDH/HRP2) card test, for malaria diagnosis in the forested belt of central India. Malar J 2008, 7: 126. 
20. Harvey SA, Jennings L, Chinyama M, Masaninga F, Mulholland K, Bell DR: Improving community health worker use of malaria rapid diagnostic tests in Zambia: package instructions, job aid and job aid-plus-training. Malar J 2008, 7: 160.

21. Rennie W, Phetsouvanh R, Lupisan S, Vanisaveth V, Hongvanthong B, Phompida S, Alday P, Fulache M, Lumagui R, Jorgensen P, Bell D, Harvey S: Minimising human error in malaria rapid diagnosis: clarity of written instructions and health worker performance. Trans $R$ Soc Trop Med Hyg 2007, 101: 9-18.

22. Millipore technical publications: Rapid Lateral Flow Test Strips. Considerations for Product Development. 2002.

[http://www.millipore.com/publications.nsf/a73664f9f981af8c852569b9005b4eee/348ee7096d93 729b85256bf40066a40d/\$FILE/tb500en00.pdf] Accessed 4 May 2010.

23. Schleicher \& Schuell Bioscience. Diagnostic components. 2004.

[http://www.cosmobio.com.ar/docs/DCSection.pdf] Accessed 4 May 2010.

24. van Dijk DP, Gillet P, Vlieghe E, Cnops L, Van Esbroeck M, Jacobs J: Evaluation of the Palutop+4 malaria rapid diagnostic test in a non-endemic setting. Malar J 2009, 8: 293.

25. van Dijk DP, Gillet P, Vlieghe E, Cnops L, Van Esbroeck M, Jacobs J: Evaluation of the Immunoquick+4 malaria rapid diagnostic test in a non-endemic setting. Eur $J$ Clin Microbiol Infect Dis 2010, 29: 577-583.

26. Gillet P, van Dijk DP, Bottieau E, Cnops L, Van Esbroeck M, Jacobs J: Test characteristics of the SD FK80 Plasmodium falciparum/Plasmodium vivax malaria rapid diagnostic test in a non-endemic setting. Malar J 2009, 8: 262.

27. Seidahmed OM, Mohamedein MM, Elsir AA, Ali FT, Malik eF, Ahmed ES: End-user errors in applying two malaria rapid diagnostic tests in a remote area of Sudan. Trop Med Int Health 2008, 13: 406-409.

28. Veron V, Carme B: Recovery and use of Plasmodium DNA from malaria rapid diagnostic tests. Am J Trop Med Hyg 2006, 74: 941-943.

29. World Health Organization: How to use a Rapid Diagnostic Test (RDT): A guide for training at a village and clinic level. 2008.

[http://www.wpro.who.int/sites/rdt/using_rdts/training/rdt training falciparum.htm] Accessed 4 May 2010. 


\section{Chapter V}

\section{External quality assessment on the use of} malaria rapid diagnostic tests in a nonendemic setting 


\title{
External quality assessment on the use of malaria rapid diagnostic tests in a non-endemic setting
}

\begin{abstract}
Background

Malaria rapid diagnostic tests (RDTs) are increasingly used as a tool for the diagnosis of malaria, both in endemic and in non-endemic settings. The present study reports the results of an external quality assessment (EQA) session on RDTs in a non-endemic setting.
\end{abstract}

\section{Methods}

After validation of antigen stability during shipment at room temperature, three clinical samples and a questionnaire were sent to clinical laboratories in Belgium and the Grand Duchy of Luxembourg using malaria RDTs. Participants were asked to report the results of the RDTs as observations (visibility of the RDT control and test lines) and interpretations (report as formulated to the clinician). In addition, participants were invited to fill in a questionnaire on the place of RDTs in the diagnostic strategy of malaria.

\section{Results}

A total of 128/133 (96.2\%) of clinical laboratories using RDTs participated. Six three-band and one four-band RDT brands were used. Analytical errors were rare and included (i) not recognizing invalid RDT results (1.6\%) and (ii) missing the diagnosis of Plasmodium falciparum (0.8\%). Minor errors were related to RDT test result interpretation and included (i) reporting "RDT positive" without species identification in the case of $P$. falciparum and non-falciparum species $(16.9 \%$ and $6.5 \%$ respectively) and (ii) adding incorrect comments to the report (3.2\%). Some of these errors were related to incorrect RDT package insert instructions such as (i) not reporting the possibility of mixed species infection in the case of $P$. falciparum and Plasmodium vivax (35.5\% and $18.5 \%$ respectively) and (ii) the interpretation of $P$. vivax instead of non-falciparum species at the presence of a pan-species antigen line $(4.0 \%)$. According to the questionnaire, $48.8 \%$ of participants processed $\leq 20$ requests for malaria diagnosis in 2009. During opening hours, 93.6\% of 125 participants used RDTs as an adjunct to microscopy but outside opening hours, nearly one third of 113 participants relied on RDTs as the primary $(4.4 \%)$ or the single tool $(25.7 \%)$ for malaria diagnosis.

\section{Conclusion}

In this non-endemic setting, errors in RDT performance were mainly related to RDT test line interpretations, partly due to incorrect package insert instructions. The reliance on RDTs as the primary or the single tool for the diagnosis of malaria outside opening hours is of concern and should be avoided. 


\section{Background}

Malaria rapid diagnostic tests (RDTs) are increasingly used as a diagnostic tool in both malaria endemic and non-endemic settings. RDTs detect Plasmodium parasites in blood by antibody-antigen reactions on a nitrocellulose strip, which become visible as cherry-red lines. Different formats exist: two-band RDTs are mostly designed to detect Plasmodium falciparum. They display a control line and a test line, which targets either histidine-rich protein-2 (HRP-2) or P. falciparum-specific parasite lactate dehydrogenase (Pf-pLDH). Three- and four-band RDTs display a control line and two or three test lines, one targeting a $P$. falciparum specific antigen, a second line targeting antigens common to the four species, such as panPlasmodium-specific lactate parasite dehydrogenase (pan-pLDH) or aldolase, and in case of the four band RDTs, a third line which targets Plasmodium vivax-specific pLDH (Pv-pLDH).

The use of RDTs is rapidly expanding: in 2007, more than 70,000,000 tests were performed and more than 80 brands were world-wide marketed [1]. In malariaendemic regions, RDTs are currently rolled out by national malaria control programs as a tool for parasite based diagnosis [1]. In non-endemic settings, where microscopic expertise is lacking due to low incidence, RDTs have been reported to perform accurately and even better as compared to microscopy [2,3]. Despite their robust design and their ease of use, RDTs are not fail-proof and quality problems have been identified at the level of production, transport and storage as well as at the level of the end-user's performance [4-10]. Initiatives for quality assurance along the route from design to end-user have been inspired by the World Health Organization (WHO). At the level of product performance there have been the two evaluation rounds of RDTs by WHO/Foundation for Innovative New Diagnostics (FIND) $[11,12]$. At the level of production and distribution, WHO has set up a comprehensive quality control strategy for production, transport and product control in national reference laboratories as well as a lot testing program [13,14]. With regard to the end-user's performance, there has been the redaction of generic job aids [15,16] and the development of stable positive controls [17]. Till now, external quality assessment (EQA) sessions on the use of RDTs have not been organized. A national survey in the UK has, however, highlighted the need for such EQA sessions from the part of clinical laboratories [18]. In light of these reasons, the present EQA was organized.

\section{Methods}

\section{Participants, samples and questionnaire}

The present EQA session was organized among clinical laboratories in Belgium and the Grand Duchy of Luxembourg which had declared to be interested in EQA 
sessions on malaria rapid diagnostic tests when subscribing to the EQA session "Blood Parasites" or "Microbiology" organized by the Institute of Public Health, Brussels, Belgium. In a survey afterwards, the non-subscribing laboratories were addressed to ask whether they use RDTs or not in the diagnosis of malaria.

The EQA panel consisted of three samples of EDTA-anticoagulated blood: one sample with $P$. falciparum, another with no evidence of Plasmodium and a third one with $P$. vivax (Table 1). They were obtained from patients suspected of malaria presenting at the Institute of Tropical Medicine (ITM). After initial analysis and diagnosis, samples were stored at $4^{\circ} \mathrm{C}$ for a maximum of 48 hours and subsequently aliquoted in $150 \mu \mathrm{l}$-fractions which were stored at $-70^{\circ} \mathrm{C}$. Total durations of storage at $-70^{\circ} \mathrm{C}$ till EQA shipment were 612,249 and 240 days for samples 1,2 and 3 respectively.

Table 1: Clinical information and parasite density of the embedded samples of the EQA session.

\begin{tabular}{|c|c|c|}
\hline Sample number & History & Species, parasite density \\
\hline 1 & Pregnant woman, Nigeria. & P. falciparum, $53,024 / \mu 1=1 \%$ \\
\hline 2 & $\begin{array}{l}\text { NGO volunteer, Burkina Faso, } \\
\text { treated for malaria } 4 \text { weeks ago. }\end{array}$ & No Plasmodium detected. \\
\hline 3 & $\begin{array}{c}\text { Traveler: Democratic Republic of the } \\
\text { Congo, Haiti. }\end{array}$ & P. vivax $, 3,251 / \mu 1=0.06 \%$ \\
\hline
\end{tabular}

Diagnosis of malaria, species identification and determination of parasite density were done by microscopy and confirmed by PCR. According to standard practice at ITM, thick and thin blood films were stained with Giemsa ( $\mathrm{pH} \mathrm{8.0)}$ and examined by light microscopy using a $\times 500$ magnification [19]. Parasite densities were estimated by counting asexual parasites against 200 white blood cells (WBC) in thick blood films and converting this number to parasites/ $\mu$ l using the actual WBC count [8]. Parasite densities are further in this text expressed as counts (of asexual parasites) $/ \mu 1$ (of whole blood), with 50,000 red blood cells/ $\mu$ l set as $1 \%$ of red blood cells [8]. Species identification was confirmed by Plasmodium-specific PCR [20].

In addition to the samples, a questionnaire on the performance of RDTs in clinical laboratories was prepared. This questionnaire was based on a previous survey performed in the UK [18] and addressed issues of frequency of requests of malaria diagnosis, ease of use and the place of RDTs in the diagnostic strategy. 
Table 2: Overview of malaria RDT brands used by the participants $(n=128)$.

\begin{tabular}{|c|c|c|c|c|}
\hline Manufacturer & Malaria RDT & Format & Target antigens & $\begin{array}{c}\text { Numbers of } \\
\text { participants } \\
(\%)\end{array}$ \\
\hline $\begin{array}{l}\text { Inverness Medical } \\
\text { Binax, Inc., } \\
\text { Scarborough, Maine, } \\
\text { USA }\end{array}$ & $\frac{\text { BinaxNOW@ }}{\text { Malaria Test }}$ & $\begin{array}{l}\text { Card box } \\
\text { Three-band }\end{array}$ & $\begin{array}{l}\text { HRP-2 + } \\
\text { Aldolase }\end{array}$ & $54(42.1)$ \\
\hline $\begin{array}{l}\text { All Diag, Strasbourg, } \\
\text { France }\end{array}$ & Palutop $+4 ®$ & $\begin{array}{l}\text { Cassette } \\
\text { Four-band }\end{array}$ & $\begin{array}{l}\text { HRP-2 + Pv- } \\
\text { pLDH + pan- } \\
\text { pLDH }\end{array}$ & $26(20.3)$ \\
\hline $\begin{array}{l}\text { DiaMed AG, Cressier } \\
\text { s/Morat Switzerland }\end{array}$ & OptiMal-IT & $\begin{array}{c}\text { Hybrid } \\
\text { dipstick } \\
\text { Three-band }\end{array}$ & $\begin{array}{l}\text { Pf-pLDH + } \\
\text { pan-pLDH }\end{array}$ & $23(18.0)$ \\
\hline
\end{tabular}

Access Bio Inc, New Jersey, USA

\section{$\underline{\text { CareStart }}^{\mathrm{TM}}$ \\ Malaria $\mathrm{pLDH} / \mathrm{HRP} 2$}

Combo test

Cassette

Three-band

$\underline{\text { SD Bioline }}$ Malaria Ag Pf/Pan FK 60
Cassette

Three-band
HRP-2 + pan-pLDH
HRP-2 + pan-pLDH
Standard Diagnostics Korea
Malaria ( $P$.
falciparum /
pan) Test

Cassette

Three-band
HRP-2 +

pan-pLDH
$11(8.6)$

$1(0.8)$

\section{Validation of shipment and questionnaire}

For validation of the shipment at room temperature (in particular the antigen stability), a try-out session was performed among the members of the IPH referee committee, which consists of a panel of laboratory professionals in charge of piloting EQA sessions. Aliquots of the samples at ITM were retrieved, allowed to thaw and processed at room temperature. The samples were tested against a panel of RDTs available in Belgium, as listed in Table 2. Two of these brands were not 
available at the time of try-out session (Cypress and Ultimed); they were performed at the moment of the formal EQA session. Aliquots were packaged according to the UN 3373 recommendations and transported the same day by car to a regular IPH referee committee meeting during which they were distributed: each member $(\mathrm{n}=$ 9) received two packages, one for analysis by his/her laboratory and another to be sent back by regular post mail to ITM. The latter package contained a temperature logger (Escort data loggers $®$, Buchanan, Virginia United States), making it possible to monitor the temperature during transport and shipment. Upon arrival at ITM, the returned packages with the samples were stored at $4^{\circ} \mathrm{C}$ for a maximum of 48 hours and RDT testing was repeated. The test line results observed upon testing these returned aliquots were compared to those that had been obtained in fresh samples.

All RDTs were performed according to the instructions of the manufacturer, except that the transfer straws or loops supplied in the RDT kits were replaced by a transfer pipette (Finnpipette, Helsinki, Finland). Readings were carried out at daylight assisted by a standard electric bulb by two subsequent blinded observers, and test line intensities were recorded [19]. RDT kits had been stored between $18^{\circ} \mathrm{C}$ and $24^{\circ} \mathrm{C}$ before use. Laboratory diagnosis of malaria at ITM is accredited in accordance with the requirements of the standard NBN EN ISO 15189:2007.

The questionnaire and the instructions were drafted in Dutch. They were translated into French (both French and Dutch are national languages in Belgium) by a native French speaking professional and both versions were again checked by a professional not involved in the study preparations. Comments and feedback to the questionnaire and instructions raised during the try-out session were addressed during a next IPH referee committee meeting and a final version of the questionnaire was approved.

\section{Data analysis}

Participants were asked to state the RDT brand they used and to report their results in terms of observations (i.e. the presence of control and test lines) and interpretations. Interpretations referred to the final diagnosis: participants were offered a free-text option and were invited to submit their answer formulated as a report to the clinician. Participants submitted the results on-line via the IPH-EQA website or sent them by post mail to IPH. The results were converted (answers through the website) or encoded (forms sent by post mail) in an Excel® database (Microsoft Corporation, Redmond, Washington USA).

For analysis, the results of the RDT tests, and answers to the questionnaire were reviewed and grouped. As the intended denominator consisted of the number of participants, only the first RDT was considered in case a participant used more than one RDT brand. Primarily, the interpretations of RDT results (report to the clinician) 
were considered. In case of an unexpected or incorrect result, the observations (results of control and test lines) were retrieved in order to distinguish between interpretative, analytical and clerical errors. In case a final result in terms of interpretations was not clearly stated (e.g. phrasings such as "result depends on microscopy"), it was not included for analysis. For interpretation and scoring of the RDT results, major, minor and very minor errors were defined based on relevance and impact on patient care, they are as listed in Table 3. Continuous variables were assessed for significance using the Student's t-test.

Table 3: Score for EQA test results, considered as "report to the clinician".

Correct Correct diagnosis and correct report.

Very minor error - Not diagnosing or reporting the possibility of a mixed infection, with non-falciparum species as the disregarded species.

Minor error

- $\quad$ Missing the diagnosis of non-falciparum species.

- $\quad$ Reporting "positive" when information on confirmation/ruling out of $P$. falciparum is available.

- $\quad$ Reporting P. vivax in stead of non-falciparum species.

- $\quad$ Correct result but with incorrect comment.

Major error

- Invalid RDT test result not recognized.

- $\quad$ Diagnosis of P. falciparum missed.

- $\quad$ P. falciparum diagnosed or reported as non-falciparum species.

- $\quad$ Non-falciparum species diagnosed or reported as P. falciparum.

- Negative sample diagnosed or reported as "positive".

\section{Additional analyses}

In an attempt to explain for some RDT reporting errors, the package inserts of the RDT kits were reviewed for the "interpretation" section.

To have an idea about the expected parasite densities of malaria samples in the present non-endemic setting, the parasite densities of the samples processed at ITM were retrieved. These samples were obtained from patients attending the outpatient clinic at ITM or sent by Belgian laboratories to ITM for confirmation in the scope of the national reference function. Only the first sample per patient was considered, for the period from January 2000 to June 2010. 


\section{Results}

\section{EQA sessions}

The try-out EQA session was performed on June $24^{\text {th }}, 2009$, during summer season. Eight out of nine temperature monitored packages arrived at ITM. The median duration of transport of the samples was 52 hours (range $49-144$ hours). Mean and maximum temperatures during shipment were $23.6^{\circ} \mathrm{C}$ (ranges $22.6^{\circ} \mathrm{C}-25.2^{\circ} \mathrm{C}$ ) and $28.7^{\circ} \mathrm{C}\left(25.9^{\circ} \mathrm{C}-32.1^{\circ} \mathrm{C}\right)$. The temperatures during shipment had exceeded $25.0^{\circ} \mathrm{C}$ for a median duration of 630 minutes (range 350 - 6,380 minutes). The formal EQA session was organized on February $22^{\text {nd }} 2010$, during winter season.

Results of the try-out testing were identical to those obtained by RDT testing of fresh samples for the three shipped samples and all the tested RDT brands. The results of Cypress and Ultimed (performed on stored samples at the time of the formal EQA session) were in line with the expected results (HRP-2 and pan-pLDH test lines visible in sample 1 , no test lines visible in sample 2, and pan-pLDH line visible in sample 3 ).

\section{An overview of participants and malaria RDTs used}

The total number of laboratories subscribing to the Belgian EQA "Microbiology" in 2009 - 2010 was 183 . A total of 128 subscribed to the session on malaria RDTs. When surveyed, 50 of the 55 non-subscribing laboratories declared not to perform RDTs as part of malaria diagnosis. In other words, 133/183 (72.7\%) of clinical laboratories offering malaria diagnosis were using RDTs at the time of EQA, and $128(96.2 \%)$ of them participated to the present EQA session on malaria RDTs.

Table 2 lists the different RDT brands used by the 128 participants, matched with their format and target antigens. Two participants used more than one RDT brand: one of them used two additional brands (SD Bioline and a $P$. vivax-pLDH specific RDT), and another participant used Palutop as part of an internal evaluation procedure. The results of these additional RDTs were not considered for analysis.

\section{RDT results for the samples}

Table 4 displays the results for sample 1 ( $P$. falciparum, parasite density of $53,024 / \mu 1)$. The expected result was: " $P$. falciparum, a mixed infection with $P$. vivax, Plasmodium ovale or Plasmodium malariae (or in case of the four-band Palutop: "P. ovale or P. malariae") cannot be excluded". Four participants did not give a final result in terms of interpretation and report to the clinician. Their observations of test lines were correct, but they were not included for analysis. The final denominator consisted of 124 participants. Only a single major error was observed: one sample was reported as negative. However, the observations of test lines reported for this sample were correct (presence of HRP-2 and pan-pLDH lines), suggesting an administrative error. Less than half of the participants scored this sample correct. One third committed the very minor error of not mentioning the 
possibility of a mixed infection with non-falciparum species. The reporting of only 'positive' without mentioning the presence of $P$. falciparum was observed in $16.9 \%$ of reports and was considered as a minor error.

Table 4: Results for sample 1: P. falciparum sample. Eligible answers of 124 participants were included.

\begin{tabular}{|c|c|c|c|c|c|c|c|}
\hline \multirow[b]{2}{*}{ Reported result } & \multicolumn{7}{|c|}{ RDT brand } \\
\hline & Binax & Palutop & Optimal & CareStart & $\begin{array}{c}\text { SD } \\
\text { Bioline }\end{array}$ & $\begin{array}{l}\text { Ultimed/ } \\
\text { Cypress }\end{array}$ & $\begin{array}{c}\text { Total } \\
(\%)\end{array}$ \\
\hline Negative* & & 1 & & & & & $\begin{array}{c}1 \\
(0.8)\end{array}$ \\
\hline Positive $^{\dagger}$ & 13 & 1 & 2 & 1 & 3 & 1 & $\begin{array}{c}21 \\
(16.9)\end{array}$ \\
\hline P. falciparum $^{\ddagger}$ & 1 & 22 & 10 & 7 & 4 & & $\begin{array}{c}44 \\
(35.5)\end{array}$ \\
\hline $\begin{array}{l}\text { P. falciparum } \\
\text { or mixed } \\
\text { infection }\end{array}$ & 37 & 2 & 11 & 4 & 4 & & $\begin{array}{c}58 \\
(46.8)\end{array}$ \\
\hline
\end{tabular}

*major, $\uparrow$ minor and $\$$ very minor errors, see definitions in Table 3.

Table 5 shows the result for sample 2 (no Plasmodium detected). The expected result was "negative" or "no Plasmodium antigen detected". Three participants did not answer a final result in terms of interpretation and report to the clinician. Their observations of test lines were correct, but they were not included for analysis. The final denominator consisted of 125 participants. Three major errors occurred. One participant reported " $P$. falciparum or mixed infection". The corresponding observations of the test line results were supporting this result, i.e. strong line intensities for both Pf-pLDH and pan-pLDH lines. Two other participants reported the absence of the control line for this sample but failed to report the test result as invalid. Twenty-four participants added a comment to the report: most comments were valuable adjuncts pointing to the need of repeating the RDT (and microscopy) in case of a negative test result and a persistent suspicion of malaria. Four participants added comments that were considered as not correct. One of these comments was raised by two participants, it stated - in case of negative result and persistent suspicion - "to repeat the RDT at the next peak of fever". 
Table 5: Results for sample 2: Plasmodium negative sample. Eligible answers of 125 participants were included.

\begin{tabular}{|c|c|c|c|c|c|c|c|}
\hline \multirow[b]{2}{*}{ Reported result } & \multicolumn{7}{|c|}{ RDT brand } \\
\hline & Binax & Palutop & Optimal & CareStart & $\begin{array}{c}\text { SD } \\
\text { Bioline }\end{array}$ & $\begin{array}{l}\text { Ultimed/ } \\
\text { Cypress }\end{array}$ & $\begin{array}{c}\text { Total } \\
(\%)\end{array}$ \\
\hline $\begin{array}{l}P . \text { falciparum or } \\
\text { mixed infection* }\end{array}$ & & & 1 & & & & $\begin{array}{c}1 \\
(0.8)\end{array}$ \\
\hline $\begin{array}{l}\text { Absence of control } \\
\text { line not reported as } \\
\text { invalid* }\end{array}$ & & & 1 & & 1 & & $\begin{array}{c}2 \\
(1.6)\end{array}$ \\
\hline $\begin{array}{l}\text { Negative }+ \text { comment } \\
\text { which is NOT } \\
\text { correct }^{\dagger}\end{array}$ & 1 & 1 & 1 & 1 & & & $\begin{array}{c}4 \\
(3.2)\end{array}$ \\
\hline $\begin{array}{l}\text { Negative + Correct } \\
\text { comment }\end{array}$ & 5 & 4 & 2 & 3 & 5 & 1 & $\begin{array}{c}20 \\
(16.0)\end{array}$ \\
\hline Negative & 45 & 21 & 18 & 8 & 5 & 1 & $\begin{array}{c}98 \\
(78.4)\end{array}$ \\
\hline
\end{tabular}

*major and $\uparrow$ minor errors, see definitions in Table 3.

Table 6 displays the results of the third sample ( $P$. vivax, parasite density $3,251 / \mu 1)$. The expected result was "P. vivax, $P$. ovale or $P$. malariae" or "non-falciparum malaria". In case of the four-band Palutop the correct result was: "P. vivax, mixed infection with $P$. ovale and $P$. malariae not excluded". Three participants did not answer a final result in terms of interpretation and report to the clinician. Their observations of test lines were correct, but they were not included for analysis. The final denominator consisted of 125 participants. None of the participants using Binax observed a test line and therefore all of them missed the expected diagnosis. Minor errors observed were the reports of simply "positive" or "pan-species" (n= $13,10.5 \%$ ), without mentioning the absence of $P$. falciparum infection. The report of " $P$. vivax" in case of Palutop was considered a very minor error since the possibility of mixed infection was not mentioned. In case of CareStart, the answer "P. vivax" was of note since this brand does not include a $P$. vivax-specific test line. 
Table 6: Results for sample 3: P. vivax sample. Eligible answers of 124 participants were included.

\begin{tabular}{|c|c|c|c|c|c|c|c|}
\hline \multirow[b]{2}{*}{ Reported result } & \multicolumn{7}{|c|}{ RDT brand } \\
\hline & Binax & Palutop & Optimal & CareStart & SD Bioline & $\begin{array}{l}\text { Ultimed/ } \\
\text { Cypress }\end{array}$ & Total $(\%)$ \\
\hline Negative $^{\dagger}$ & 51 & & & & & & $51(41.1)$ \\
\hline Positive $^{\dagger}$ & & & 1 & 1 & 2 & 1 & $5(4.0)$ \\
\hline Pan-species $^{\dagger}$ & & 1 & 2 & 2 & 3 & & $8(6.5)$ \\
\hline P. vivax & & $23^{\ddagger}$ & & $5^{\dagger}$ & & & $28(22.6)$ \\
\hline $\begin{array}{l}P . \text { vivax; } P \text {. ovale } \\
\text { and } P \text {. malariae } \\
\text { not excluded }\end{array}$ & & 2 & & & & & $2(1.6)$ \\
\hline $\begin{array}{l}\text { Pan-species, } \\
\text { not P. falciparum }\end{array}$ & & & 20 & 4 & 6 & & $30(24.2)$ \\
\hline
\end{tabular}

\section{Results of the questionnaire}

Table 7 lists the numbers of requests for malaria diagnosis processed by each laboratory in 2009 , matched to the number of laboratory staff. In line with the low number of requests were the low numbers of RDT tests performed in 2009: 56.8\% (71/125) of participants declared to have processed 20 tests or less, another third $(44 / 125,35.2 \%)$ mentioned between 20 and 100 RDTs. More than three quarters of participants $(97 / 120,80.8 \%)$ replied that RDTs had improved the diagnosis of malaria in their setting. 
Table 7: Cross tabulation of the numbers of laboratory staff involved in malaria diagnosis versus the numbers of requests for malaria diagnosis in 2009.

\begin{tabular}{|c|c|c|c|c|c|}
\hline \multirow{2}{*}{$\begin{array}{l}\text { Numbers of requests for } \\
\text { malaria diagnosis in } 2009\end{array}$} & \multicolumn{5}{|c|}{ Numbers of laboratory staff performing malaria diagnosis } \\
\hline & $0-5$ & $6-10$ & $11-20$ & $>20$ & Total \\
\hline $0-10$ & 4 & 8 & 16 & 3 & 31 \\
\hline $11-20$ & 11 & 7 & 11 & 1 & 30 \\
\hline $21-100$ & 4 & 16 & 17 & 12 & 49 \\
\hline $101-500$ & 4 & 4 & 5 & 1 & 14 \\
\hline$>500$ & & 1 & & & 1 \\
\hline Total & 23 & 36 & 49 & 17 & 125 \\
\hline
\end{tabular}

Table 8 lists the ease of use for each of the RDT brands expressed on a scale from 0 to 10 . Although the median scores did not differ much and differences did not reach statistical significance, there was a wider range with a tendency to lower scores for Binax and, to a lesser extent, OptiMAL.

Table 8: Ease of use of the different malaria RDTs expressed as a score.

\begin{tabular}{|c|c|c|c|}
\hline RDT brand & $\begin{array}{c}\text { Numbers of laboratories using this } \\
\text { brand }\end{array}$ & Median Score & Range \\
\hline Binax & 52 & 8 & $2-10$ \\
\hline OptiMAL & 23 & 8 & $6-9$ \\
\hline Palutop & 25 & 9 & $7-10$ \\
\hline CareStart & 12 & 9 & $8-10$ \\
\hline SD Bioline & 11 & 9 & $8-10$ \\
\hline
\end{tabular}

Tables 9 and 10 list the diagnostic strategies during and outside opening hours (weekend and night shifts). A total of 125 participants gave eligible answers on their diagnostic strategy during opening hours, among them there were 113 participants who also offered malaria diagnosis outside opening hours. Of note, five participants noted that they do not perform RDTs on follow-up samples. The vast majority $(95.2 \%)$ of participants used RDTs as a complement or adjunct to microscopy during opening hours, but only $62.8 \%$ did so outside opening hours. Moreover, outside opening hours, $31.1 \%$ of them relied on RDTs as the primary $(4.4 \%)$ or the single tool $(25.7 \%)$ for malaria diagnosis. In a minority (approximately 5\%) of 
laboratories, the decision of performing a RDT either alone or in conjunction with microscopy was left to the attending clinician.

Table 9: Strategy of malaria diagnosis during opening hours as reported by 125 participants.

\section{Diagnostic strategy of malaria during opening hours}

Microscopy + always RDT

Microscopy + RDT for confirmation

Microscopy + RDT if requested by the clinician

Microscopy and/or RDT, depending on the request by the clinician

RDT, if positive or in case of doubt: + microscopy
Numbers of participants $(\%)$

Table 10: Strategy of malaria diagnosis outside opening hours as reported by 113 participants.

\section{Diagnostic strategy of malaria outside opening hours}

Microscopy + always RDT

Microscopy + RDT for confirmation

Microscopy + RDT if requested by the clinician

Microscopy alone

Microscopy and/or RDT, depending on the request by the clinician

RDT: if RDT positive, microscopy is done instantly; if RDT is negative, microscopy is done the next day

RDT + microscopy next day

RDT: if positive or in case of doubt: + microscopy

RDT alone, no microscopy
Numbers of participants (\%)

63 (55.8)

$8(7.1)$

16 (14.2)

13 (11.5) 


\section{Additional analyses}

To generate an idea about the parasite densities of patients presenting in Belgium and in the Grand Duchy of Luxembourg, archived data were reviewed at ITM. Table 11 lists the parasite densities of the samples submitted to ITM for each species, for a 10-year period (January 2000 - June 2010).

The frequency of not reporting the possibility of a mixed infection in the case of $P$. falciparum (sample 1, 35.3\%) and P. vivax (22.6\%, sample 3) was striking. In sample 1, half of these errors occurred with Palutop and represented the vast majority (22/26) of reports obtained with this brand. For this reason, the package insert of this kit was analysed: for the combination of the "Pf" (HRP-2) and the "Pan" (pan-pLDH) lines, Palutop instructions mentioned "P. falciparum" without adding the possibility of a mixed infection with $P$. ovale or $P$. malariae. A similar omission was noted for the $P$. vivax interpretation: the possibility of a co-infection with $P$. malariae and $P$. ovale was not mentioned (Figure 1). OptiMAL kit's instructions neither mentioned the possibility of a mixed infection in the case of the diagnosis " $P$. falciparum" (Figure 2).

Likewise, there were errors in the interpretation of test lines in the CareStart package insert. From this brand, two versions were available: a version with individually wrapped tests ("Single Kits", "Lab in a pack") and a regular laboratory kit. For the interpretation of a single pan-pLDH line, the former mentioned "pan-species" and the latter " $P$. vivax", thereby explaining for the unexpected reporting of " $P$. vivax" by five participants (Figure 3, Table 6).

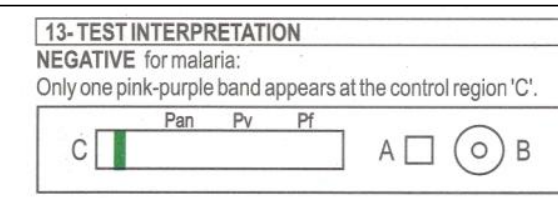

POSITIVE for malaria:

P. falciparum malaria: In addition to the control band, a pink-purple band appears at the 'Pf' and 'Pan' regions respectively.

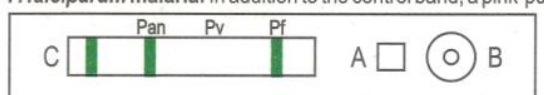

P.vivax malaria: In addition to the control band, a pink-purple band appears at 'Pv' and 'Pan' regions respectively.

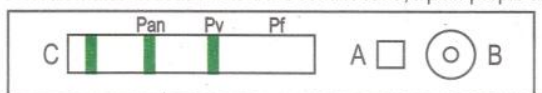

Other species: In addition to the control band, one pink-purple band appears only at 'Pan' region.

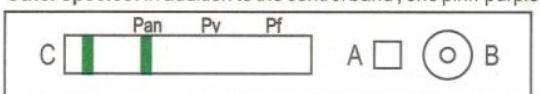

Mixed infection: In addition to the control band, a pink-purple band appears at 'Pf', 'Pv' and 'Pan' regions respectively.

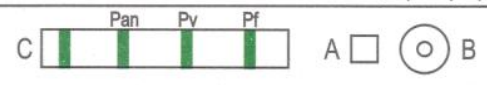

Figure 1: Package insert of Palutop, test interpretation. The instructions do not mention the possibility of a mixed infection in case of " $P$. falciparum malaria" and " $P$. vivax malaria". 


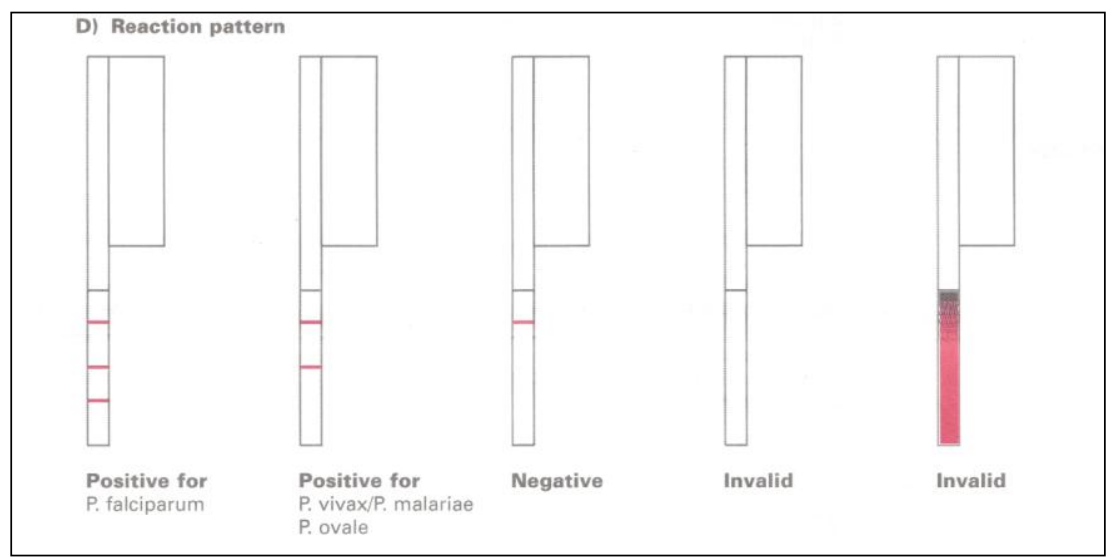

Figure 2: Package insert of OptiMAL, test interpretation. The instructions do not mention the possibility of a mixed infection in case of "Positive for P. falciparum".

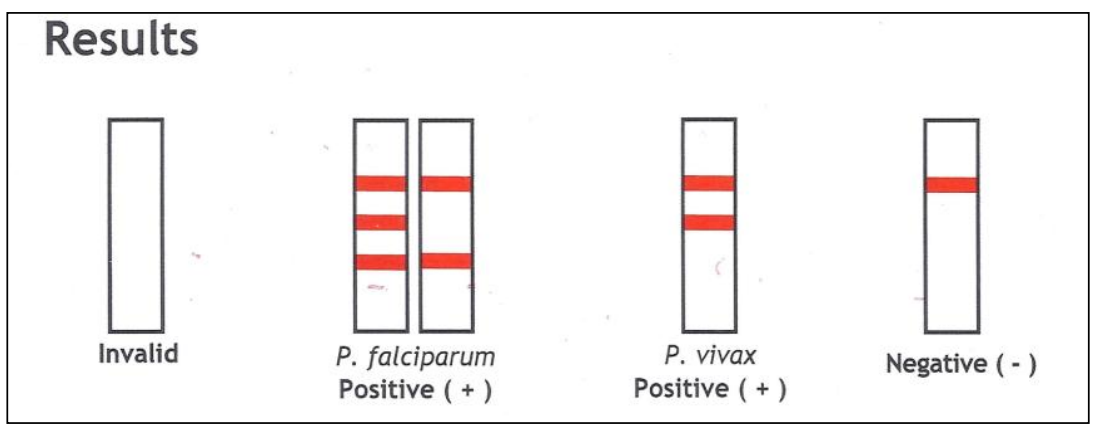

Figure 3: Package insert of CareStart "Single Kit". Test interpretation stating "P. vivax" instead of "non-falciparum species" when a single pan-pLDH line is visible.

\section{Discussion}

The present study reports the findings of an EQA session on malaria RDTs in a nonendemic setting. Major analytical errors were rare. Minor errors occurred frequently and were related to interpretation or reporting of test results (e.g. reporting "RDT positive" without species identification when this was possible based on RDT test line result). Errors in interpretations (e.g. the possibility of mixed infections and the identification of the non-falciparum species) were embedded in the package insert instructions of some RDTs. Nearly half (48.8\%) of participants received $\leq 20$ requests for malaria diagnosis in 2009. During opening hours, $93.6 \%$ of participants 
used RDTs as an adjunct to microscopy whereas outside opening hours, nearly one third of them relied on RDTs as primary or single tool for malaria diagnosis.

As far as known, this is the first report of an EQA on malaria RDTs. It should be noted that the present EQA session suffered from the weaknesses inherent to the EQA method. For instance, only a few samples were offered, precluding comparison of diagnostic characteristics of the different RDT brands. Furthermore, participants were alerted to the importance of the samples and probably might have devoted more attention to them than to routinely submitted samples. Finally, a single EQA session such as the present one will only offer a cross sectional idea about the performances of the participants. On the other hand, the method of EQA has its strengths: it provides an educational stimulus to the participants, allowing them to boost their self-confidence and to show their credibility. Furthermore, EQA sessions including the present one also provide information to the health authorities: they provide insights into the participants' performance levels, may trace problems in test kits and methods and they can survey the use of diagnostic algorithms [21]. A particular strength of the present EQA was its representativeness: in Belgium, subscription to EQA sessions is mandatory for clinical laboratories and the registration fee covers all sessions. This particular condition explains for the high coverage rate (96.2\% of all laboratories performing RDTs) of this EQA session.

The try-out EQA session validated the use of clinical samples after storage and shipment. According to $\mathrm{WHO}$ findings, antigen stability in sample panels is preserved during storage at $-70^{\circ} \mathrm{C}$ for 20 months; in addition, storage at $4^{\circ} \mathrm{C}$ before freezing samples does not impair panel quality nor does storage at room temperature for up to 60 days [22]. In light of these findings and the present results, extension of EQA sessions to other settings such as endemic field settings might be feasible. The only difference with fresh clinical samples is the hemolysis caused by freezing: the additional hemolysis however does not interfere with RDT processing but contributes to swift clearance of the background on the nitrocellulose strip [23].

In the present EQA session, analytical errors were rare. Two major errors were observed. The single missed diagnosis of $P$. falciparum (sample 1) was related to an administrative rather than to an analytical error; the unexpected single " $P$. falciparum" report for one out of 26 Palutop tests (sample 2) might also be related to a clerical error (mixing up specimens or laboratory forms) although the reported test line results were in line with the correct result. More of concern was the missed interpretation of invalid test results by two participants, which was considered as a major error: in the absence of a visible control line, test results are not reliable and the test should be repeated. Invalid test results occur rarely $(<0.5 \%$ of samples tested) but consistently [24-27] and laboratory staff should be alert to this phenomenon. 
Interpretation or reporting of RDT results was more subject to errors: simply reporting "positive" without mentioning the species in the case of $P$. falciparum (Sample 1, 16.9\%) and non-falciparum (Sample 3, 4.0\%) or reporting "pan-species" without mentioning that P. falciparum was not involved (Sample 3, 6.5\%) was considered as a minor error: all relevant information from the RDT results, in particular the presence or absence of the potentially fatal P. falciparum, should be exploited.

Sample 2 did not contain Plasmodium antigen. Although this was a confirmed malaria-negative sample correctly identified by $97.6 \%$ of participants, it should be noted that false-negative RDT results may occur. Reasons for false-negative RDT results in a non-endemic setting are low parasite densities, polymorphisms in HRP-2 and the prozone effect. Low parasite densities are by far the most frequent cause of false-negative RDTs: for $P$. falciparum, they occur more frequently below the threshold of 100 parasites/ $\mu 1$, but at this density non-immune travelers can present with symptoms [19,26-29]. From Table 11 can be read that $P$. falciparum samples with parasite densities below $100 / \mu 1$ can be expected in about $10 \%$ of samples. In such cases - and as raised by some participants as a comment to their report of sample 2 - repeated testing after $8-12$ hours is advised, for up to four consecutive samplings [30,31]. Of note, the comment of awaiting a next peak of fever for repeating the RDT was considered as a minor error: although there are variations in antigen production during the cycle, there is no clear relation between the concentration of antigens and any particular moment of the cycle, yet the peak of fever [29] and a periodic fever pattern does not occur in most of the P. falciparum infections. Polymorphisms in HRP-2 may give rise to variations that are less likely to be picked up by current RDTs; they are geographically confined to the AsiaPacific and South-American regions [32-34]. For the prozone effect, high antigen concentrations block all available binding sites of both the detection and the capture antibodies, thereby hindering test line generation. It occurs at high parasite densities of $P$. falciparum and presents mostly as test lines with spuriously low intensity, although complete negative results do occur. It tends to occur in HRP-2 based but not in Pf-pLDH based tests [35].

The failure of Binax to detect $P$. vivax in sample 3 was not considered as a major error in light of the ability of RDTs to detect non-falciparum species. In addition, EQA sessions are not designed as a side-to-side comparison of different brands nor as an evaluation of RDT test characteristics. However, it demonstrates the lower sensitivity of RDTs to detect non-falciparum species as compared to the detection of $P$. falciparum [19,26-29,36]. For Binax (in its most recent generation), compiled sensitivity for the diagnosis of $P$. vivax has been calculated to be $68.9 \%$ [29]. In a population of returned travelers, Binax displayed sensitivities of $86.7 \%$ for pure $P$. 
vivax samples [30]. In comparison, OptiMAL which was run side-to-side in the latter study displayed a higher sensitivity for $P$. vivax (91.0\%), at the expense of a lower sensitivity for P. falciparum. Challenged to a panel of stored samples at ITM, SD Bioline, Palutop and CareStart showed overall sensitivities for the detection of P. vivax of $87.5 \%, 66.0 \%$ and $77.6 \%$ respectively $[19,26,28]$, with, as for Binax in the current sample, false-negatives not limited to low parasite densities. For $P$. ovale and $P$. malariae, reported sensitivities have been even lower [19,26,28,36]. As for $P$. falciparum, the sensitivity for the non-falciparum species is related to parasite density, with a threshold value at $500 / \mu 1$ [19,24-26,28] or even at $5,000 / \mu 1$ [12] but false negatives also occur above these densities. Parasite densities below these 500 and 5,000 threshold values occur in $23.0 \%$ and $67.6 \%$ of the non-falciparum samples in the Belgian reference laboratory for malaria (Table 11).

Table 11: Distribution of parasite densities (asexual parasites/ $\mu 1$ ) per species for the 1066 Plasmodium positive samples submitted to ITM for the period January 2000 - June 2010. (Only the first sample per patient was included).

\begin{tabular}{|c|c|c|c|c|c|}
\hline \multirow{2}{*}{$\begin{array}{c}\text { Parasite density } \\
\text { Numbers }\end{array}$} & \multicolumn{4}{|c|}{ Single infection, species } & \multirow{2}{*}{ Mixed infection ${ }^{\dagger}$} \\
\hline & P. falciparum & P. vivax & P. ovale & P. malariae & \\
\hline $0-100$ & 81 & 3 & 12 & 3 & 0 \\
\hline $101-500$ & 93 & 14 & 22 & 3 & 2 \\
\hline $501-5,000$ & 221 & 52 & 38 & 20 & 4 \\
\hline $5,001-250,000$ & 360 & 59 & 14 & 6 & 5 \\
\hline$>250,000$ & 53 & 1 & & & \\
\hline Total. & 808 & 129 & 86 & 32 & 11 \\
\hline \multicolumn{6}{|c|}{ Cumulative (\%) } \\
\hline$\leq 100$ & 10.0 & 2.3 & 14.0 & 9.4 & 0 \\
\hline$>100$ & 90.0 & 97.7 & 86.0 & 90.6 & 100 \\
\hline$>500$ & 78.5 & 86.8 & 60.5 & 81.3 & 81.8 \\
\hline$>5.000$ & 51.1 & 46.5 & 16.3 & 18.8 & 45.5 \\
\hline$>250.000$ & 6.6 & 0.01 & 0 & 0 & 0 \\
\hline
\end{tabular}

${ }^{\dagger}$ Mixed infections included $P$. falciparum infection with $P$. ovale $(\mathrm{n}=4)$ or $P$. malariae $(\mathrm{n}=5)$ and $P$. malariae infection with $P$. ovale $(\mathrm{n}=1)$ or $P$. $\operatorname{vivax}(\mathrm{n}=1)$. 
Not reporting the possibility of a mixed infection of $P$. falciparum with nonfalciparum species (Sample 1, 35.5\%) was considered a minor error, as this has no impact on the short-term patient care: in case of an additional $P$. vivax or $P$. ovale species, the persistent liver schizonts have to be eradicated with primaquine treatment, but there is time to await this information from the reference laboratory. However, it was striking that this error as well as the error of not reporting the possibility of mixed infections in the case of $P$. vivax (sample 3, 22.6\%) were embedded in the package insert instructions of Palutop and OptiMAL. Likewise there were errors on interpretation in the Carestart package insert instructions, with incorrect labeling of the test lines in both versions. Manufacturers should be encouraged to revise and adapt their instructions where needed, especially with regard to test line interpretations.

The questionnaire confirmed the low critical volume in terms of requests for malaria diagnosis: assuming a 5-10\% positivity rate for patients suspected of malaria, it is clear that laboratory technicians have little exposure to malaria-positive slides. Even in those laboratories with high numbers of requests, the number of performances per technician will be reduced by a high number of laboratory staff participating in the diagnosis. This is comparable to the situation in other countries, with about half of laboratories in the U.K. carrying out less than 100 malaria requests each year and about $10 \%$ of laboratories fewer than 10 [18]. In France, about $60 \%$ of 3,300 surveyed laboratories declared to have seen a malaria case the previous year, and only $5 \%$ made the diagnosis of malaria five times or more yearly [37].

The number of participants $(80.8 \%)$ replying that malaria RDTs had improved the diagnosis of malaria in their setting was much higher than revealed by a survey in the UK (12.6\% out of 305 respondents stated that RDTs had revised their malaria diagnosis) [18]. Although the meaning and phrasing of the latter question were different ("revised" versus "improved"), the difference in numbers is striking. It may be attributed to a growing experience with malaria RDTs in clinical laboratories.

The wider range with a tendency to lower scores for ease of use for Binax and, to a lesser extent, OptiMAL may be explained by the fact that these kits include more steps than the more recently released one-step kits such as CareStart and SD Bioline. In ITM experience as well as that of others [7,38], particular problems can arise with the use of the transfer device: without having processed high numbers of samples, it may be difficult to master the RDT kit's loops, straws or capillary tubes, which are frequently small and not user-friendly. The volume of blood used to run the test is critical: an excess of blood may increase the risk and/or the intensity of prozone effect or may mask a faint line due to a bad clearance of the strip, while a shortage of blood may decrease the sensitivity of the test.

Of particular interest are the diagnostic strategies during and outside opening hours. Whereas the vast majority of participants used RDTs as a complement or adjunct to microscopy during opening hours, the reliance on RDTs as the primary or the single 
diagnostic tool during night and weekend shifts is of concern. In the UK-survey of 2006 a similar tendency was observed: less than 5\% of 327 surveyed laboratories used exclusively a RDT during opening hours, versus $15-20 \%$ outside opening hours [18]. The extent of the potential risks as a result of this strategy may be serious especially since, according to a survey in Portugal, about half of the requests for the diagnosis of malaria arrive outside opening hours, accounting for $60 \%$ of the diagnoses [39]. In view of their strengths, RDTs are a valuable adjunct for malaria diagnosis and should - in the authors' opinion - be used together with microscopy in all cases of malaria suspicion in non-endemic settings. Conversely, RDT limitations do not justify them as the unique tool for diagnosis in non-endemic settings: microscopy is needed to recover diagnosis that may be missed by RDTs (prozone effect, non-falciparum species and low parasite densities), and also to assess signs of severity (elevated parasite densities and the presence of schizonts and haemozoin in the case of $P$. falciparum). It is important to ascertain reliable diagnosis of malaria during and outside office hours, and competent microscopy should be in reach at all times. To minimize the risk of microscopy errors and as an in-service training and feedback, all positive and doubtful samples should be submitted to the malaria reference laboratory.

The policy of leaving the decision on the choice of RDT versus microscopy to the attending clinician may result in not performing the RDT (thereby not exploiting possible information generated by the RDT) or not performing microscopy. This policy is probably related to the reimbursement system of medical costs in Belgium: national health insurance reimburses only laboratory analyses that are explicitly requested by the clinician. Hospital-based diagnostic and treatment algorithms can guide the choice and priorities of laboratory tests, but for the individual patient however, the ultimate request of RDT, microscopy or both depends on the clinician's decision.

The additional comment of five participants that they do not perform RDTs on follow-up samples is a correct policy. Indeed, HRP-2 may persist in the circulation for up to several weeks. Aldolase and pLDH depend on the living parasites and they rapidly decline after start of correct treatment, but their use is limited because they are also expressed by gametocytes [40]. Consequently, monitoring of treatment efficacy should be done by microscopy only.

\section{Conclusion}

From the present EQA session, it is clear that RDTs are an essential part of malaria diagnosis in most diagnostic laboratories in Belgium and the Grand Duchy of Luxembourg. According to the results of the present EQA, it can be concluded that analytical errors in the performance of RDTs are rare. Errors are mainly related to the interpretation and reporting of RDT results, partly due to errors in the package insert. Laboratory staff has limited exposure to malaria positive samples in this non- 
endemic setting. Whereas during opening hours, RDTs are used as a complement or adjunct to microscopy, there are about one third of participants that rely on RDTs as the primary or the single diagnostic tool outside opening hours, which should be avoided.

\section{List of abbreviations}

EDTA: Ethylene diamine tetra-acetic acid; EQA: External quality assessment; FIND: Foundation for Innovative New Diagnostics; IPH: Belgian Scientific Institute of Public Health; HRP-2: Histidine-rich protein 2; INRB: Institut National de Recherche Biomédicale; ITM: Institute of Tropical Medicine; P: Plasmodium; PanpLDH: pan Plasmodium-specific parasite lactate dehydrogenase; PCR: Polymerase chain reaction; Pf-pLDH: Plasmodium falciparum-specific parasite lactate dehydrogenase; pLDH: parasite lactate dehydrogenase; Pv-pLDH: Plasmodium vivax-specific parasite lactate dehydrogenase; RDT(s): Rapid diagnostic test(s); WHO: World Health Organization.

\section{Acknowledgements}

We would like to thank the members of the IPH referee committee for their contribution in the try-out EQA session, and the staff of the Central Laboratory of Clinical Biology (ITM, Antwerp) for technical support.

\section{References}

1. Drakeley $\mathrm{C}$, Reyburn H: Out with the old, in with the new: the utility of rapid diagnostic tests for malaria diagnosis in Africa. Trans R Soc Trop Med Hyg 2009, 103: 333-337.

2. Palmer CJ, Bonilla JA, Bruckner DA, Barnett ED, Miller NS, Haseeb MA, Masci JR, Stauffer WM: Multicenter study to evaluate the OptiMAL test for rapid diagnosis of malaria in U.S. hospitals. J Clin Microbiol 2003, 41: 5178-5182.

3. Stauffer WM, Cartwright CP, Olson DA, Juni BA, Taylor CM, Bowers SH, Hanson KL, Rosenblatt JE, Boulware DR: Diagnostic performance of rapid diagnostic tests versus blood smears for malaria in US clinical practice. Clin Infect Dis 2009, 49: 908-913.

4. Chiodini PL, Bowers K, Jorgensen P, Barnwell JW, Grady KK, Luchavez J, Moody AH, Cenizal A, Bell D: The heat stability of Plasmodium lactate dehydrogenase-based and histidine-rich protein 2-based malaria rapid diagnostic tests. Trans R Soc Trop Med Hyg 2007, 101: 331337.

5. de Oliveira AM, Skarbinski J, Ouma PO, Kariuki S, Barnwell JW, Otieno K, Onyona P, Causer LM, Laserson KF, Akhwale WS, Slutsker L, Hamel M: Performance of malaria rapid diagnostic tests as part of routine malaria case management in Kenya. Am J Trop Med Hyg 2009, 80: 470-474.

6. Jorgensen $\mathrm{P}$, Chanthap L, Rebueno A, Tsuyuoka R, Bell D: Malaria rapid diagnostic tests in tropical climates: the need for a cool chain. Am J Trop Med Hyg 2006, 74: 750-754.

7. McMorrow ML, Masanja MI, Abdulla SM, Kahigwa E, Kachur SP: Challenges in routine implementation and quality control of rapid diagnostic tests for malaria--Rufiji District, Tanzania. Am J Trop Med Hyg 2008, 79: 385-390.

8. Moody AH, Chiodini PL: Non-microscopic method for malaria diagnosis using OptiMAL IT, a second-generation dipstick for malaria pLDH antigen detection. Br J Biomed Sci 2002, 59: 228-231.

9. Rennie W, Phetsouvanh R, Lupisan S, Vanisaveth V, Hongvanthong B, Phompida S, Alday P, Fulache M, Lumagui R, Jorgensen P, Bell D, Harvey S: Minimising human error in malaria 
rapid diagnosis: clarity of written instructions and health worker performance. Trans $R$ Soc Trop Med Hyg 2007, 101: 9-18.

10. Seidahmed OM, Mohamedein MM, Elsir AA, Ali FT, Malik eF, Ahmed ES: End-user errors in applying two malaria rapid diagnostic tests in a remote area of Sudan. Trop Med Int Health 2008, 13: 406-409.

11. World Health Organization: Malaria Rapid Diagnostic Test Performance; Results of WHO product testing of malaria RDTs: Round 1 (2008). 2009.

[http://www.finddiagnostics.org/resource-centre/reports brochures/malaria-diagnostics-report2009.html]

12. World Health Organization: Malaria Rapid Diagnostic Test Performance; Results of WHO product testing of malaria RDTs: Round 2 (2009). 2010.

[http://www.finddiagnostics.org/resource-centre/reports_brochures/malaria-diagnostic-testreport-round2.html]

13. World Health Organization: Lot-testing: Using quality control parasite dilutions to test the sensitivity of malaria RDTs. 2010.

[http://www.wpro.who.int/sites/rdt/using_rdts/qa/lot testing.htm]

14. World Health Organization: Parasitological confirmation of malaria diagnosis. 2010.

[http://whqlibdoc.who.int/publications/2010/9789241599412_eng.pdf]

15. World Health Organization: How to use a Rapid Diagnostic Test (RDT): A guide for training at a village and clinic level. 2008.

[http://www.wpro.who.int/sites/rdt/using_rdts/training/rdt_training_falciparum.htm]

16. World Health Organization: RDT Instructions and Training. 2008.

[http://www.wpro.who.int/sites/rdt/using rdts/training/rdt training falciparum.htm]

17. Versteeg I, Mens PF: Development of a stable positive control to be used for quality assurance of rapid diagnostic tests for malaria. Diagn Microbiol Infect Dis 2009, 64: 256-260.

18. Chilton D, Malik AN, Armstrong M, Kettelhut M, Parker-Williams J, Chiodini PL: Use of rapid diagnostic tests for diagnosis of malaria in the UK. J Clin Pathol 2006, 59: 862-866.

19. Van der Palen M, Gillet P, Bottieau E, Cnops L, Van Esbroeck M, Jacobs J: Test characteristics of two rapid antigen detection tests (SD FK50 and SD FK60) for the diagnosis of malaria in returned travellers. Malar J 2009, 8: 90.

20. Cnops L, Jacobs J, Van Esbroeck M: Validation of a four-primer real-time PCR as a diagnostic tool for single and mixed Plasmodium infections. Clin Microbiol Infect 2010.

21. Kettelhut MM, Chiodini PL, Edwards H, Moody A: External quality assessment schemes raise standards: evidence from the UKNEQAS parasitology subschemes. J Clin Pathol 2003, 56: 927-932.

22. World Health Organization: Towards Quality Testing of Malaria Rapid Diagnostic Tests: Evidence and Methods. 2006.

[http://www.wpro.who.int/internet/resources.ashx/RDT/docs/pdf_version/web3_QARDTreport.pdf]

23. Gillet P, Mori M, Van Den Ende J, Jacobs J: Buffer substitution in malaria rapid diagnostic tests causes false-positive results. Malar J 2010, 9: 215 .

24. Gillet P, Bosselaers K, Cnops L, Bottieau E, Van Esbroeck M, Jacobs J: Evaluation of the SD FK70 malaria Ag Plasmodium vivax rapid diagnostic test in a non-endemic setting. Malar $J$ 2009, 8: 129 .

25. Gillet P, van Dijk DP, Bottieau E, Cnops L, Van Esbroeck M, Jacobs J: Test characteristics of the SD FK80 Plasmodium falciparum/Plasmodium vivax malaria rapid diagnostic test in a non-endemic setting. Malar J 2009, 8: 262.

26. van Dijk DP, Gillet P, Vlieghe E, Cnops L, Van Esbroeck M, Jacobs J: Evaluation of the Palutop+4 malaria rapid diagnostic test in a non-endemic setting. Malar J 2009, 8: 293.

27. van Dijk DP, Gillet P, Vlieghe E, Cnops L, Van Esbroeck M, Jacobs J: Evaluation of the Immunoquick+4 malaria rapid diagnostic test in a non-endemic setting. Eur $J$ Clin Microbiol Infect Dis 2010, 29: 577-583.

28. Maltha J, Gillet P, Bottieau E, Cnops L, Van Esbroeck M, Jacobs J: Evaluation of a rapid diagnostic test (CareStart Malaria HRP-2/pLDH (Pf/pan) Combo Test) for the diagnosis of malaria in a reference setting. Malar $J$ 2010, 9: 171.

29. Murray CK, Gasser RA, Jr., Magill AJ, Miller RS: Update on Rapid Diagnostic Testing for Malaria. Clin Microbiol Rev 2008, 21: 97-110.

30. Farcas GA, Zhong KJ, Lovegrove FE, Graham CM, Kain KC: Evaluation of the Binax NOW ICT test versus polymerase chain reaction and microscopy for the detection of malaria in returned travelers. Am J Trop Med Hyg 2003, 69: 589-592. 
31. Forney JR, Magill AJ, Wongsrichanalai C, Sirichaisinthop J, Bautista CT, Heppner DG, Miller RS, Ockenhouse CF, Gubanov A, Shafer R, DeWitt CC, Quino-Ascurra HA, Kester KE, Kain $\mathrm{KC}$, Walsh DS, Ballou WR, Gasser RA, Jr.: Malaria rapid diagnostic devices: performance characteristics of the ParaSight $\mathbf{F}$ device determined in a multisite field study. $J$ Clin Microbiol 2001, 39: 2884-2890.

32. Baker J, McCarthy J, Gatton M, Kyle DE, Belizario V, Luchavez J, Bell D, Cheng Q: Genetic Diversity of Plasmodium falciparum Histidine-Rich Protein 2 (PfHRP2) and Its Effect on the Performance of PfHRP2-Based Rapid Diagnostic Tests. J Infect Dis 2005, 192: 870-877.

33. Baker J, Ho MF, Pelecanos A, Gatton M, Chen N, Abdullah S, Albertini A, Ariey F, Barnwell J, Bell D, Cunningham J, Djalle D, Echeverry DF, Gamboa D, Hii J, Kyaw MP, Luchavez J, Membi C, Menard D, Murillo C, Nhem S, Ogutu B, Onyor P, Oyibo W, Wang SQ, McCarthy J, Cheng Q: Global sequence variation in the histidine-rich proteins 2 and 3 of Plasmodium falciparum: implications for the performance of malaria rapid diagnostic tests. Malar $J$ 2010, 9: 129.

34. Gamboa D, Ho MF, Bendezu J, Torres K, Chiodini PL, Barnwell JW, Incardona S, Perkins M, Bell D, McCarthy J, Cheng Q: A large proportion of $\boldsymbol{P}$. falciparum isolates in the Amazon region of Peru lack pfhrp2 and pfhrp3: implications for malaria rapid diagnostic tests. PLoS One 2010, 5: e8091.

35. Gillet P, Mori M, Van Esbroeck M, Van Den Ende J, Jacobs J: Assessment of the prozone effect in malaria rapid diagnostic tests. Malar $J$ 2009, 8: 271.

36. Marx A, Pewsner D, Egger M, Nuesch R, Bucher HC, Genton B, Hatz C, Juni P: Meta-analysis: accuracy of rapid tests for malaria in travelers returning from endemic areas. Ann Intern Med 2005, 142: 836-846.

37. Moulin F, Gendrel D: Imported malaria: diagnostic traps and rapid tests. Arch Pediatr 2009, 16 Suppl 2: S89-S92.

38. Luchavez J, Lintag ME, Coll-Black M, Baik F, Bell D: An assessment of various blood collection and transfer methods used for malaria rapid diagnostic tests. Malar J 2007, 6 : 149.

39. Hanscheid T: Current strategies to avoid misdiagnosis of malaria. Clin Microbiol Infect 2003, 9: 497-504.

40. Mueller I, Betuela I, Ginny M, Reeder JC, Genton B: The sensitivity of the OptiMAL rapid diagnostic test to the presence of Plasmodium falciparum gametocytes compromises its ability to monitor treatment outcomes in an area of Papua New Guinea in which malaria is endemic. J Clin Microbiol 2007, 45: 627-630. 
$-126-$ 


\section{Chapter VI}

Malaria rapid diagnostic kits: quality of packaging, design and labelling of boxes and components and readability and accuracy of information inserts

Gillet P, Maltha J, Hermans V, Ravinetto R, Bruggeman C, Jacobs J.

Malaria Journal 2011; 10:39.

http://www.malariajournal.com/content/10/1/39 
Malaria rapid diagnostic kits: quality of packaging, design and labelling of boxes and components and readability and accuracy of information inserts

\begin{abstract}
Background

The present study assessed malaria RDT kits for adequate and correct packaging, design and labelling of boxes and components. Information inserts were studied for readability and accuracy of information.
\end{abstract}

\title{
Methods
}

Criteria for packaging, design, labelling and information were compiled from Directive $98 / 79$ of the European Community (EC), relevant World Health Organization (WHO) documents and studies on end-users' performance of RDTs. Typography and readability level (Flesch-Kincaid grade level) were assessed.

\section{Results}

Forty-two RDT kits from 22 manufacturers were assessed, 35 of which had evidence of good manufacturing practice according to available information (i.e. CE-label affixed or inclusion in the WHO list of ISO13485:2003 certified manufacturers). Shortcomings in devices were (i) insufficient place for writing sample identification $(\mathrm{n}=40)$ and (ii) ambiguous labelling of the reading window $(\mathrm{n}=6)$. Buffer vial labels were lacking essential information $(n=24)$ or were of poor quality $(n=16)$. Information inserts had elevated readability levels (median Flesch Kincaid grade 8.9 , range $7.1-12.9$ ) and user-unfriendly typography (median font size 8 , range 5 10). Inadequacies included (i) no referral to biosafety $(n=18)$, (ii) critical differences between depicted and real devices $(n=8)$, (iii) figures with unrealistic colours $(n=4)$, (iv) incomplete information about RDT line interpretations $(n=31)$ and no data on test characteristics $(n=8)$. Other problems included (i) kit names that referred to Plasmodium vivax although targeting a pan-species Plasmodium antigen $(\mathrm{n}=4)$, (ii) not stating the identity of the pan-species antigen $(\mathrm{n}=2)$ and (iii) slight but numerous differences in names displayed on boxes, device packages and information inserts. Three CE labelled RDT kits produced outside the EC had no authorized representative affixed and the shape and relative dimensions of the CE symbol affixed did not comply with the Directive 98/79/EC. Overall, RDTs with evidence of GMP scored better compared to those without but inadequacies were observed in both groups.

\section{Conclusion}

Overall, malaria RDTs showed shortcomings in quality of construction, design and labelling of boxes, device packages, devices and buffers. Information inserts were difficult to read and lacked relevant information. 


\section{Background}

\section{The use of malaria RDTs is rapidly expanding}

Prompt parasitological confirmation by microscopy or alternatively by RDTs is recommended in all patients suspected of malaria before treatment is started [1]. As a consequence, malaria rapid diagnostic tests (RDTs) are increasingly used as a diagnostic tool in both malaria endemic and non-endemic settings: in 2007, more than 70,000,000 tests were performed [2].

Malaria RDTs are so-called immunochromatographic tests that detect Plasmodium antigens in the blood by an antigen-antibody reaction on a nitrocellulose strip. The antigen-antibody complex is conjugated to colloidal gold, and a positive result is visible as a cherry- or purple-red coloured line. Apart from a control line, there are one, two or three test lines: the so-called two-band tests comprise a control line and a single test line, and are mostly designed to diagnose Plasmodium falciparum. Their targets are either histidine-rich protein-2 (HRP-2) or P. falciparum-specific parasite lactate dehydrogenase (Pf-pLDH). Three-band RDTs display a second test line mostly targeting antigens common to the four species such as pan-Plasmodiumspecific parasite lactate dehydrogenase (pan-pLDH) or aldolase. The four-band RDTs have an additional third test line targeting Plasmodium vivax-specific $\mathrm{pLDH}$ (Pv-pLDH).

\section{Written instructions add to the correct performance and interpretation of RDTs}

RDTs are accurate and robust but they have limitations linked to design, production and distribution [3-8]. In addition, there are errors at the level of the end-user, which apply to both laboratory staff and field workers and are related to sampling, testing and interpretation of RDTs [9,10]. Clearly written instructions can add to the comprehensibility and maximize RDT kit performance [9,10]. On this basis, the World Health Organization (WHO) designed easy-to-read generic job aids [11].

During field visits in Africa, teams of the Institute of Tropical Medicine (ITM) occasionally noted shortcomings in RDT kit boxes, content and instructions. In addition, part of the interpretation errors that were observed during a recent external quality assessment (EQA) on RDTs were shown to be related to errors in the information inserts of the RDT kits used [4]. Inspection of these information inserts also revealed a large variety in layout and readability, as well as variations in the adequacy of labelling of RDT boxes and devices. 


\section{Objectives of the present study}

In view of the observations above, it was decided (i) to assess malaria RDT kits for adequate and correct design, construction and labelling of boxes and components, and (ii) to study the readability and accuracy of their information inserts.

\section{Methods}

\section{Selection of RDT kits}

Malaria RDTs marketed as devices consisting of cassettes, cardboard boxes and hybrids (nitrocellulose strips to be dipped into plastic wells) were selected. They were checked for the presence of the CE label and evidence of good manufacturing practice (GMP) based on their inclusion in the WHO lists of RDT manufacturers and distributors complying with ISO13485:2003 or US FDA 21 CFR 820 production norms [12].

As this study was not intended to score RDTs individually, it was decided not to display the RDT brand and kit names, in line with previous comparative studies assessing RDTs [3-5,13].

\section{Criteria used for RDT kit assessment and procedure}

For packaging, design and labelling, assessment criteria were compiled from requirements listed in regulatory documents such as the Directive 98/79/EC and the European Community (EC) as well as relevant WHO documents [14-17]. Criteria for information inserts and device design from studies on end-users' performance and RDT instructions were pooled [5,7,9,10,18-24]. Inadequacies were defined as listed in Table 1.

\section{RDT kit package, device package, device and buffer vial}

The RDT kit packages were assessed for type (box versus plastic bag), material (simple and plasticized cardboard) and the presence and quality of the printed information. Information displayed on the package considered as essential included the RDT kits and manufacturer's names, expiry date, number of tests included, storage requirements and a reminder to read the instructions before use. A referral to the intended use of the RDT kit was looked for, either by the RDT kit name or by an additional text. The expiry date mentioned on the box was matched with those of the other RDT kit components. For CE labelled RDT kits produced by companies outside the European Economic Area, the affixing of the so-called authorized representative of the company in the EC (EC-REP) was assessed. Kits were assessed for sampling material needed included or not. 
Table 1: Number of RDTs $(n=42)$ with inadequacies in malaria RDT boxes, device packages, devices, buffer vials and package inserts*.

Items considered to be inadequate

Number

\section{Box: construction and design}

Materials: plastic bag or simple cardboard (not humidity-resistant)

No labels, no printed information or labels not humidity-resistant

Differences in name on device packaging, device, buffer and information insert

Box: information displayed

No EC-REP mentioned on CE labelled RDTs, although required $(\mathrm{n}=25)$

RDT kit's name nor additional information refer to intended use

RDT kit's name incorrectly refers to $P$. vivax instead of non-falciparum species $(\mathrm{n}=29)$

Kit components not displayed

Essential information lacking: expiry date, numbers of tests included, storage conditions

\section{Kit contents:}

Capillary sampling system (lancet and alcohol swap) not included or not optionally included

Blood transfer system (capillary, pipette or tube) not included

Device package and content: construction and design

Material not humidity-resistant

No desiccant or desiccant without saturation indicator

Device package and content: information displayed

Essential information lacking: expiry date, lot number, test kit name

No warning label "do not swallow" on desiccant

\section{Device: construction and design}

Space for sample identification too small or not writable with standard pen (felt pen needed)

No or incomplete RDT name on the device

$29(69.0)$

No reading label or simultaneous presence two reading labels consisting of symbols only

\section{Buffer: construction and design}

Buffer vial not leak proof

Label does not stick well to the vial, prints are not humidity-resistant $(n=40)$

\section{Buffer: information displayed}

Essential information lacking: expiry date, lot number, storage conditions, correct RDT kit's name $(\mathrm{n}=41)$

No instructions included on how to pierce the buffer vial dropper $(n=15)$

\section{Package insert: information}

Absence of date of release or version number

\section{Package insert: content}

Identity of target antigens not clearly mentioned

No referral to biosafety precautions (gloves, safe waste disposal, etc.)

Major differences between depicted and real device $(n=40)$

Use of figures with unrealistic colours (e.g. control and test lines depicted as green)

No data on test characteristics (sensitivity, specificity)

\footnotetext{
$*$ Total number of RDT kits $=42$ unless otherwise stated.
} 
The package of the test device was checked for quality (humidity-proof material) and essential information including RDT kit name, lot number and expiry date. In addition, the desiccant was checked for composition, warning label and presence of colour indicator.

The RDT devices (cassette or cardboard housing the nitrocellulose strip) were assessed for clearness of design and construction including referral to the RDT kit's name. The space allocated for sample identification was evaluated for dimensions and ease of writing. A space of minimal $0.5 \mathrm{~cm}$ height and $4 \mathrm{~cm}$ wide was considered as adequate for handwriting of sample identification. The labelling of buffer wells, sample wells and reading windows including the places of appearance of the control and test lines (further referred to as reading label) were assessed for visibility and unambiguous interpretation.

The buffer vials were assessed for leak-proof closure, and their labels for quality of adherence and print. The information displayed on the label was assessed for the presence of RDT kit name, lot number, expiry date and storage conditions.

\section{RDT information insert}

RDT kits were checked for the presence of an information insert and a job aids (short procedure version), of which date of release and version number were assessed.

Layout and figures. The figures were counted and their dimensions measured. Their total surface area was calculated and expressed as a percentage of the total surface of the information insert. The figures were assessed for their concept (pure black and white versus use of colours) and conformity with the real devices.

Typography. The font size of the predominant letter type used (excluding the bibliography section) was measured in Cicero using a typometer (Rotring-werke Riepe KG, Hamburg, Germany) as the "kp" distance from the top of the highest ascender (top of the lower case letter $\mathrm{k}$ ) to the bottom of the lowest descender (bottom of the lower case letter $\mathrm{p}$ ). The opening of the characters was visually assessed for the characters " $c$, o and a", by covering them for their lower two-thirds and checking whether they were still correctly readable (open letter type) versus read as an "o" (closed letter type). The interline spacing was assessed by measuring in Cicero with a typometer the distance between the base line of two successive rows and then subtracting the font size. Fonts of open letter types and interline spacing equal or larger than 2 are better readable compared to fonts of closed letter types and interline spacing smaller than 2, especially at larger text columns. For patient education materials and health instructions, font sizes of 12 or larger are recommended $[25,26]$. 
Readability level. For assessment of the readability level, the sessions about blood sampling, procedure and interpretation in the English text version were copied or retyped in Microsoft Word (Microsoft Corp., Redmond, WA, U.S.A.) and checked for correct spelling and syntax construction. Follow-up editing was performed as described elsewhere [27]. Next, the text fragments were copied into an on-line readability assessment tool, which generates different reading indices [28]. The Flesch-Kincaid grade level [29] was calculated. This grade-level expresses the U.S. grade-level equivalency of the skills required to read a particular document. For patient education materials and health related information, the recommended level is $\leq 6^{\text {th }}$ grade level $[25,30]$.

Accuracy and relevance of information. The following items were actively looked for: description of the RDT test principle, target antigens, listing of required materials provided and not, description of sampling procedures and biosafety precautions. The RDT test procedures were studied with reference to common errors made by end-users in the field (Table 2) [5,9-11,18,19,23]. The interpretation section was assessed for the complete description of invalid results and Plasmodium species differentiation as well as for listing causes of false negative and false positive results. The description of test characteristics was assessed for mentioning the diagnostic accuracies related to the different Plasmodium species and parasite densities. Bibliographic references were checked for relevance with regard to RDT performance in general and information on the RDT kit's performance in particular.

\section{Assessment, data registration and statistical analysis}

Two observers trained in the use of RDTs independently assessed the RDTs according to the described criteria. Discrepant observations were discussed together with the other investigators and a consensus was reached. Data were registered in an Excel sheet (Microsoft Corp., Redmond, WA, U.S.A.). 
Table 2: Number of RDT information inserts $(n=40)$ addressing critical steps in procedure and interpretation.

\section{Items addressed in procedure section}

Bring the RDT device and buffer to room temperature

Check the integrity of the device package

Check expiry date

Use the device immediately after opening

Place the device on a level surface

Check the desiccant for signs of exposure to humidity

Write down sample identification

Wipe finger with alcohol

Allow the finger to dry before pricking

Hold the transfer device (loop, straw) vertical

Hold the buffer vial vertical

Do not to use another buffer than the one provided with the kit

Use an adequate light source for reading

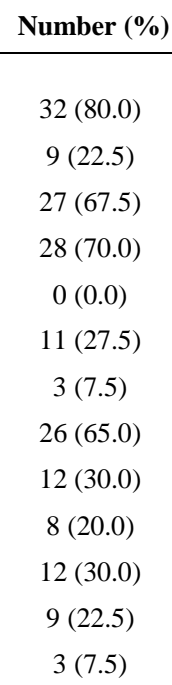

Number $(\%)$

$32(80.0)$

$9(22.5)$

$27(67.5)$

$28(70.0)$

$0(0.0)$

$11(27.5)$

$3(7.5)$

$26(65.0)$

$12(30.0)$

$8(20.0)$

$12(30.0)$

$9(22.5)$

$3(7.5)$

\section{Number $(\%)$}

$12(30.0)$

$31(77.5)$

$8(20.0)$

$11(27.5)$

$19(47.5)$

$1(2.5)$

To repeat the test in case of a negative RDT result and persistent suspicion of malaria is mentioned

\section{Results}

\section{Panel of RDT kits}

For the purpose of this study, 51 RDT kits were ordered at 29 companies. Seven companies (representing nine RDT kits) did not reply despite several reminders. The final panel consisted of 42 RDT kits from 22 companies. Nearly all (39/42, 93\%) RDT kit formats were cassettes, further there were one cardboard and two hybrid kits. Two RDT kits consisted of individually wrapped RDT packages containing all materials for a single test (cassette, disinfectant, lancet and buffer). They will be further referred to as "Single RDT kits". Table 3 lists the RDTs according to their evidence of GMP. Table 1 lists the number of the RDTs with inadequacies in boxes, devices, buffer vials and information inserts. 
Table 3: Overview of the RDT kits evaluated in the present study.

\begin{tabular}{|c|c|c|c|c|c|}
\hline \multirow[b]{2}{*}{ RDT format } & \multirow[b]{2}{*}{$\begin{array}{c}\text { Plasmodium antigens } \\
\text { targeted }\end{array}$} & \multirow[b]{2}{*}{ Number } & \multicolumn{3}{|c|}{ Evidence of GMP } \\
\hline & & & $\begin{array}{c}\mathrm{CE} \\
\text { mark }\end{array}$ & $\begin{array}{c}\text { WHO } \\
\text { list }^{\dagger}\end{array}$ & Total \\
\hline \multirow[t]{3}{*}{ Two band } & HRP-2 & 7 & 4 & 5 & 7 \\
\hline & pan-pLDH & 1 & 1 & 0 & 1 \\
\hline & Pv-pLDH & 1 & 1 & 0 & 1 \\
\hline \multirow[t]{4}{*}{ Three band } & HRP-2, pan-pLDH & 11 & 5 & 7 & 9 \\
\hline & HRP-2, aldolase ${ }^{\ddagger}$ & 5 & $5 *$ & 5 & 5 \\
\hline & HRP-2, Pv-pLDH & 4 & 1 & 1 & 2 \\
\hline & Pf-pLDH, pan-pLDH & 6 & 5 & 6 & 6 \\
\hline Four band & HRP-2, Pv-pLDH, pan-pLDH & 7 & 3 & 5 & 6 \\
\hline
\end{tabular}

\section{RTD kit package, device and buffer vial}

RDT kit package. Thirty-eight RDT kits arrived as cardboard boxes; four kits arrived in plastic bags. Two of these plastic bags contained a cardboard box to be folded by the end-user, resulting in a total of 40 boxes and two plastic bags as the package of use on the bench. All but one box displayed an indication in the RDT kit's name or in the test description that the RDT kit was intended for malaria diagnosis. The two plastic bags did not display any information. A company name was listed on all the 40 boxes, but for 12 kits, it was not clearly mentioned whether this name represented the manufacturer or the distributor.

The lot number and expiry date were listed on all boxes. One of the Single RDT kits showed both lot number and expiry date on the outer box containing the single packages, but not on the single packages themselves. There were no discrepancies between the expiry dates on the RDT kit box and those of the contents except for two buffer vials with expiry dates extending those printed on the RDT kit box. 
The number of tests included and a reminder to read the instructions before use were not displayed on four and ten boxes respectively. All the 40 boxes showed information on storage temperature requirements, by written text, symbols or both. Apart from a single symbol, i.e. a penguin expressing "do not freeze", all symbols were internationally recognized symbols complying with EN 980:2008 or FDA 2004, 21 CFR 809.10 and 21 CFR Parts 610 and 660. Capillary blood sampling systems were included in eight kits and proposed as optional in ten other kits. Blood transfer systems were missing in three kits.

Device packages. Four of 42 device packages were not made of humidity-resistant material. Most of the packages were easy to open by tearing a pre-cut lid of the package. However, for three packages, scissors had to be used to open the packages properly.

A desiccant was present in all but one package. Three of the 41 desiccants did not show a warning that the desiccant was harmful: two of them (from one manufacturer) were tablets looking like drug pills. Seventeen desiccants (including the two tablets) had no colour indicator of humidity saturation.

RDT devices. Space for writing was too small in 40 of the 42 devices (Figure 1). For two cassettes, a felt pen was required as a standard pen failed to mark.

Most cassettes (35/39) had separate wells for sample and buffer application, four had a single well for sample and buffer application. There was no uniform labelling of the wells: for instance characters "S" and "A" were used randomly for the sample well, buffer well and combined sample/buffer well (Figure 2). Fifteen cassettes showed at the distal end a window or holes that might be confused with a sample or buffer well (Figure 1 and 2).

The reading label was indicated with acronyms $(n=20)$, characters or numbers. Acronyms included abbreviations such as "Pf" or "pan", they were printed on the plastic housing or on a label and were well readable. Characters such as " $\mathrm{C}$ " (control line) and " $T$ " and numbers were embedded in the plastic housings and were more difficult to distinguish (Figure 2). In one cassette, characters were printed on a label, which was not well fixed (Figure 1). In 14 cassettes, two labels were displayed at either side of the reading window (Figure 2), and one three-band cassette had no reading label at all (Figure 2). 


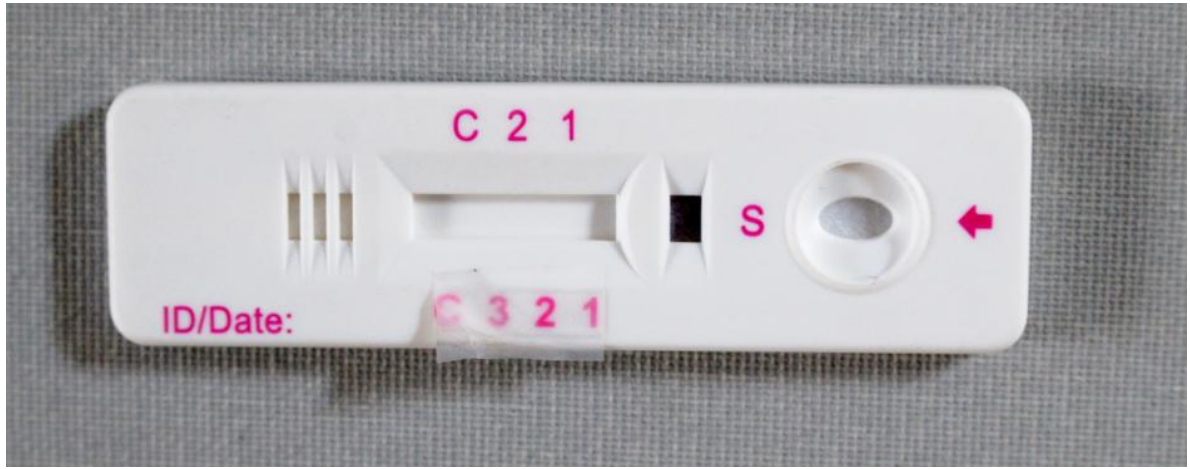

Figure 1: Four-band RDT. The allocated place for writing sample identification is too small. The grid at the left hand may be confused with a sample well. There are two different reading labels at each side of the reading window, of which the lower one is printed on a label that is not well fixed.

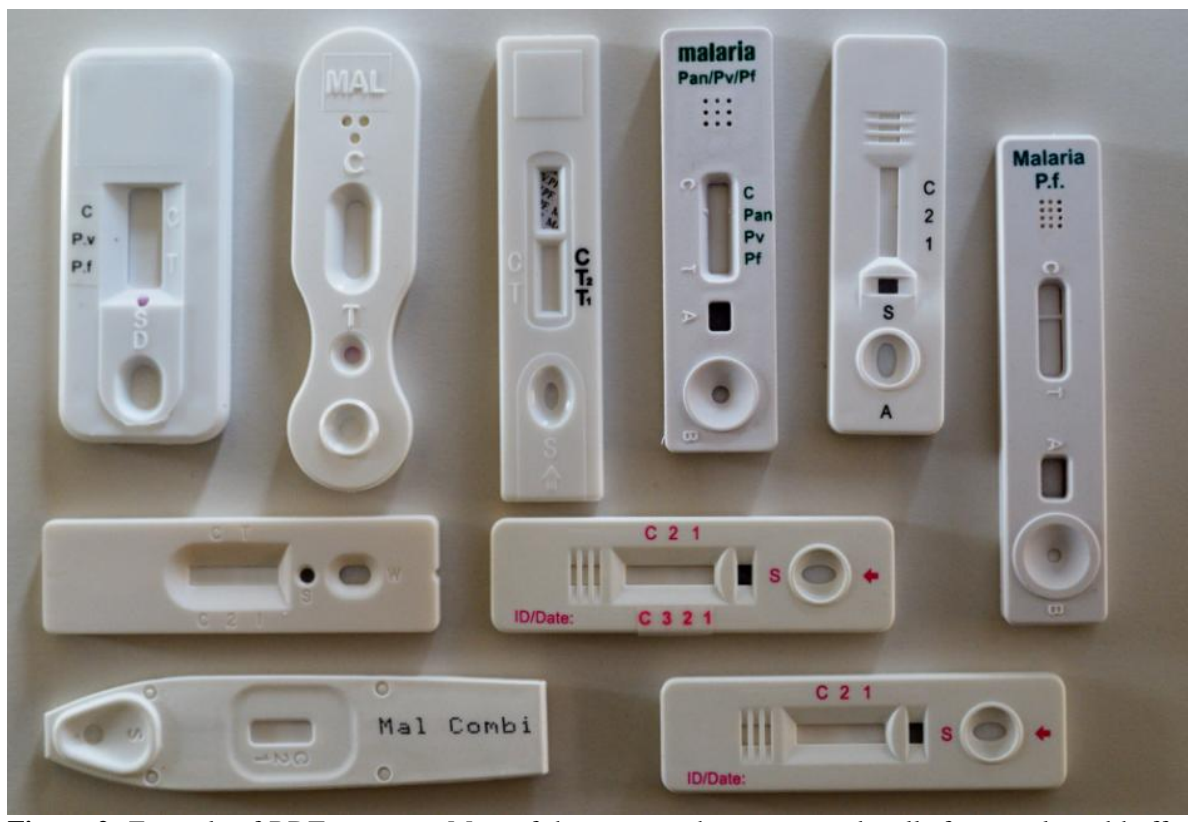

Figure 2: Example of RDT cassettes. Most of the cassettes have separated wells for sample and buffer application. There is no uniform labelling of the wells: different characters (e.g. "S", "A") are used randomly for the sample well, buffer well and combined sample/buffer well. The reading labels are indicated with acronyms, characters or numbers.

RDT buffer vials. The two Single kit RDTs contained a small buffer plastic ampoule in each device package, which were too small to display information. Fifteen buffer vials required clockwise tightening the vial cap to pierce the dropper vial nozzle, but for five of them, this was not mentioned in the information insert. For 13 vials, the label was not well fixed and the printed information on three of these labels was not humidity-resistant. Lot number and expiry date were not listed on five vials and storage conditions were missing on 10 vials. 


\section{RDT information insert}

The information inserts of the two Single RDT kits were not considered: one of them contained a simple job aids explaining the procedure by figures only, the other contained a shortened version of the information insert of the same RDT marketed as laboratory kit. All of the remaining 40 RDT kits contained an information insert of which seven had an additional job aids. Either version number or date of issue was missing in 11 and 13 of them; in five, both were missing.

Layout and figures. All 40 inserts included figures. The median number of figures per information insert was 8.5 (range $2-25$ ) and figures accounted for a median surface ratio of $7.2 \%$ (range $0.4 \%-33 \%$ ) of the entire insert. The median size of the figures was $2.4 \times 2.0 \mathrm{~cm}$, the smallest and largest figure measured respectively $1.0 \times$ $0.3 \mathrm{~cm}$ and $6.7 \times 8.0 \mathrm{~cm}$.

All inserts used figures to illustrate the interpretation section. Other figures depicted blood sampling $(n=17)$, application of sample and buffer $(n=21)$ and a clock indicating the correct reading delay $(n=10)$. Fourteen inserts used red colour to indicate control and test lines but in four, they were pictured green or blue. Most inserts $(n=35)$ showed differences between depicted and real devices of which some were major, such as discrepancies between characters used for sample and buffer well identification $(n=4)$ and differences in labelling of the reading window $(n=5)$ (Figures 3 and 4). One insert mentioned a reading delay of 15 minutes, but the illustration mentioned a reading delay of 20 minutes (Figure 5).

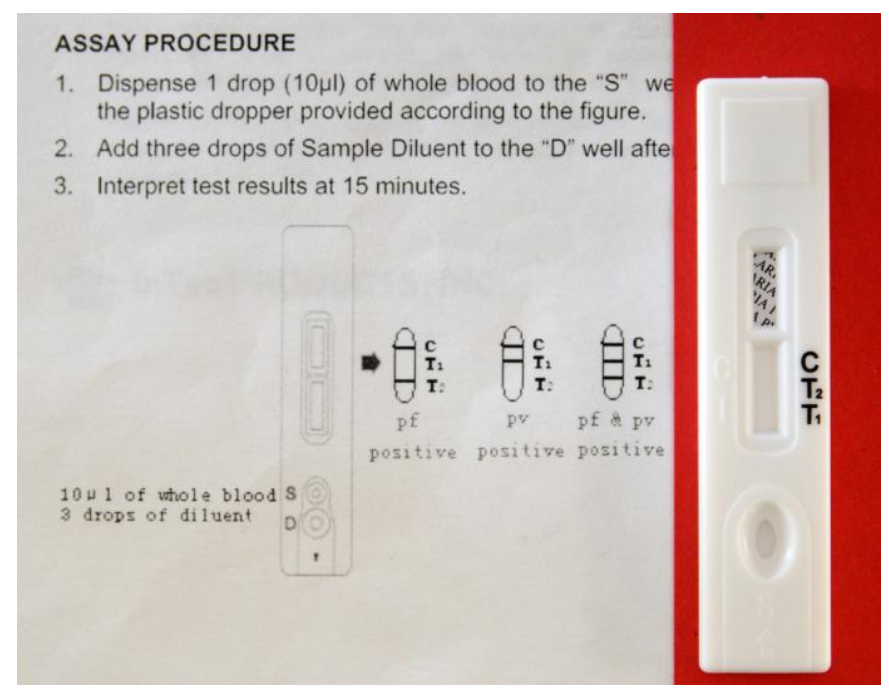

Figure 3: Interpretation section of the information insert and cassette of a Plasmodium falciparum / Plasmodium vivax RDT. The real device has a single sample/buffer whereas the depicted one displays separate wells. The characters used for the reading label on the illustration are inverted compared to the real device. 
INTERPRETATION OF THE RESULTS

\section{REACTIVE}
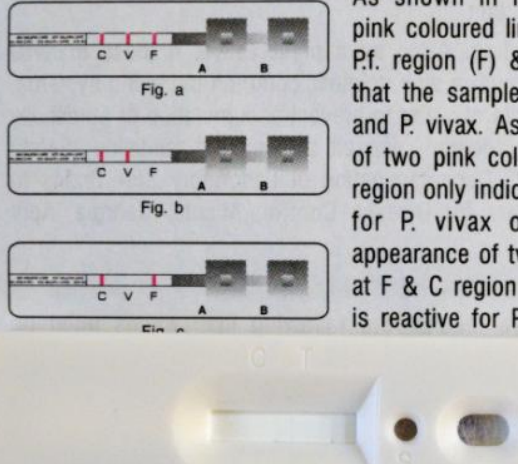

jative result the test

resuit slivulu ve ieau viny at cu mumutos. " uiv vuru. of pLDH/HRP-2 in the sample is very high, only test line may be observed. This is due to Hook's effect. Such samples should be diluted $1: 10$ or $1: 20$ in negative blood (Human) \& again re-run the test, Diluted sample should show both control \& test line. In case, if control line does not appear or is faint dilute the sample further.

Figure 4: Interpretation section of the information insert and cassette of a $P$. falciparum/P. vivax RDT. Shape and labels of wells and reading window are different between the real and the depicted device. Characters are embedded in the plastic housing and poorly discernable. The text is correct and complete (even the prozone effect and how to deal with it) but less readable (Flesch-Kincaid grade level 9.1).

\section{Wait for the red line(s) to appear. The test line should be read at 15 minutes.}

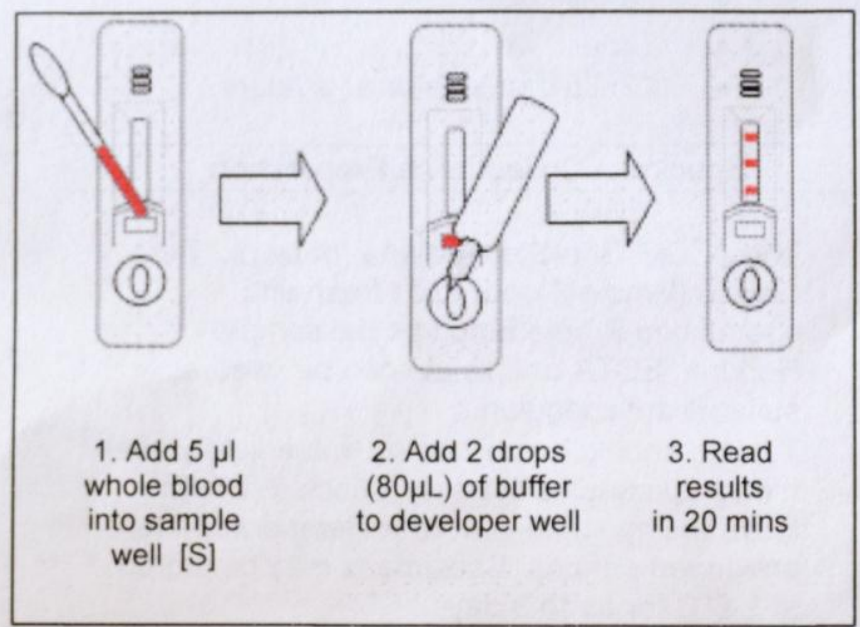

Figure 5: Information insert of a three-band RDT, procedure section. There is a discrepancy between the reading time mentioned in the text compared to that showed on the illustration. 
Typographic features and readability. Figure 6 displays the font size and line spacing of the information inserts. Median font size was $8 \pm 1.3$, none of them exceeded 10. User-unfriendly typographic features included combinations of font sizes of eight or smaller with a closed letter type $(n=10)$ or with line spacing lower than two and more than 12 words per line $(\mathrm{n}=14)$ (Figure 7). Median readability level was grade 8.9 (range $7.1-12.9$ ) and 18 and four of the inserts' readability levels were above grade 9 and 10 respectively (Figure 8). Readability levels of the job aids were also high (median 8.5, range $5.1-9.4$ ), and six out of seven exceeded the readability level of the most recent WHO job aids. Five inserts showed prints of very poor quality hindering reading of the text (Figure 9).

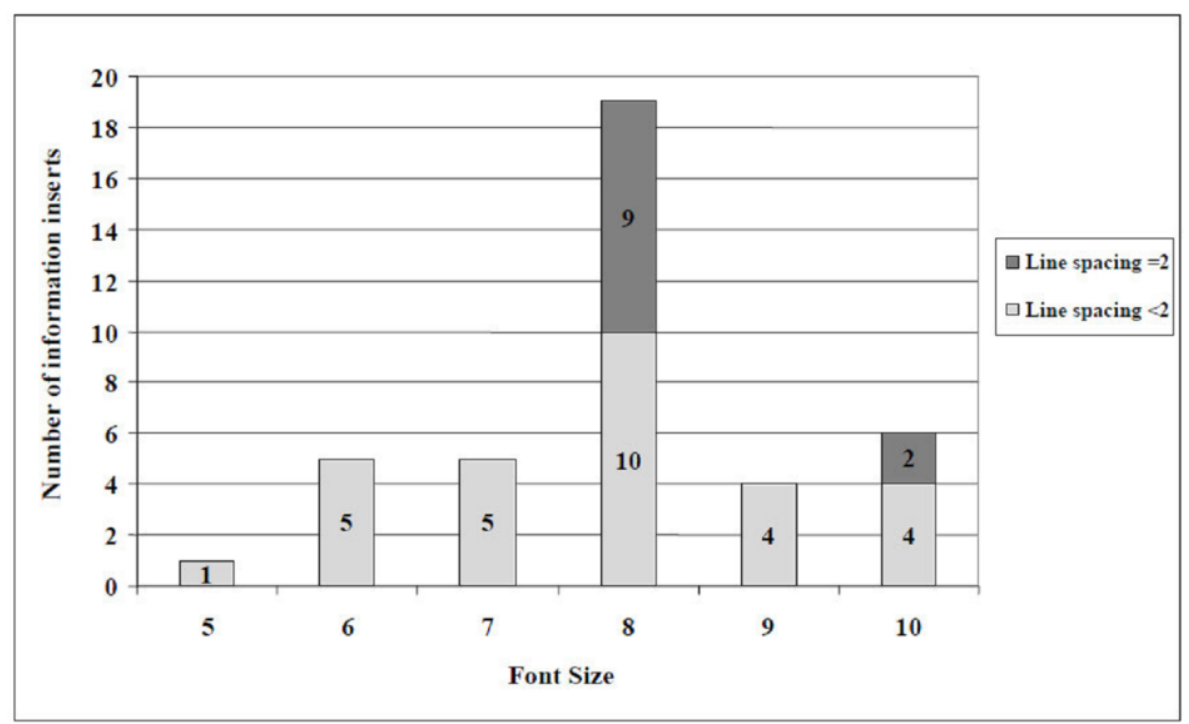

Figure 6: Typographic features of RDT information inserts $(n=40)$ : font sizes and line spacing. 


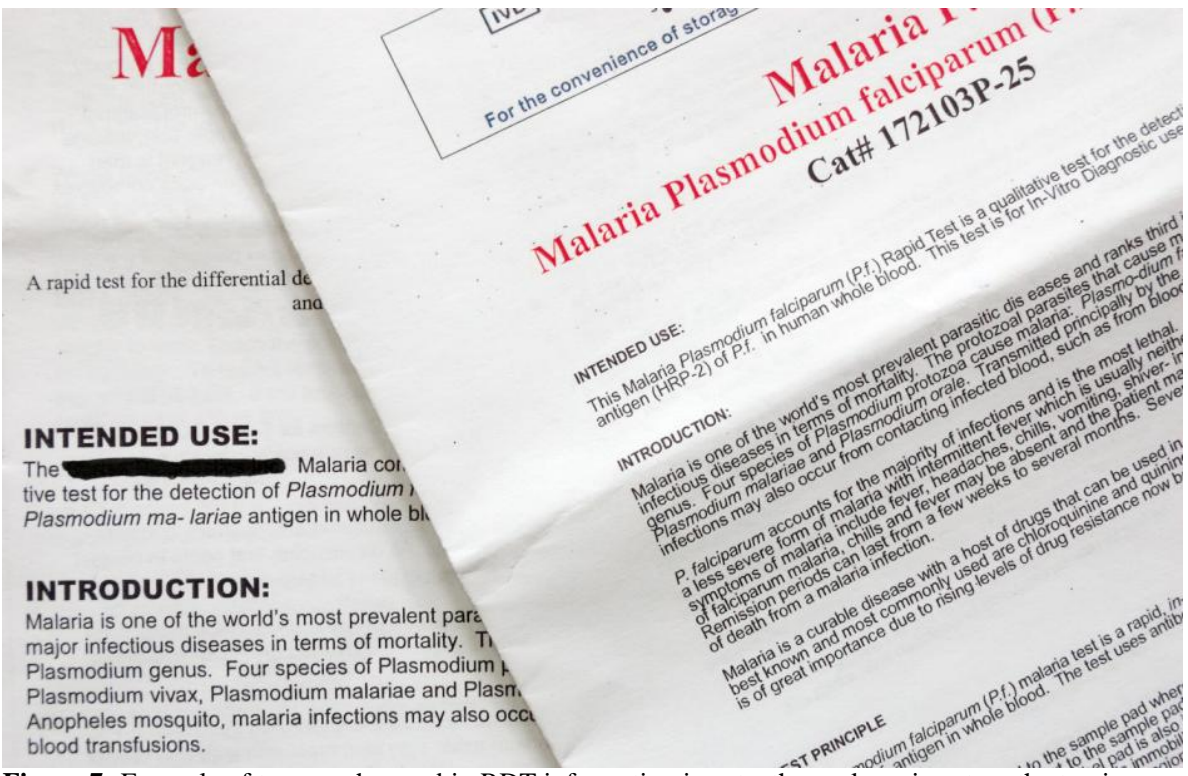

Figure 7: Example of typography used in RDT information inserts: the package insert on the top is userunfriendly (font size 6, line spacing 0.5, close letter type, average number of words per line 21, FleschKincaid grade 9.5). The package insert on the background, from the same company but for another RDT uses a better typography (font size 8 , line spacing 2 , open letter type, average number of words per line 14), but the readability is still elevated (Flesch-Kincaid grade 9.8).

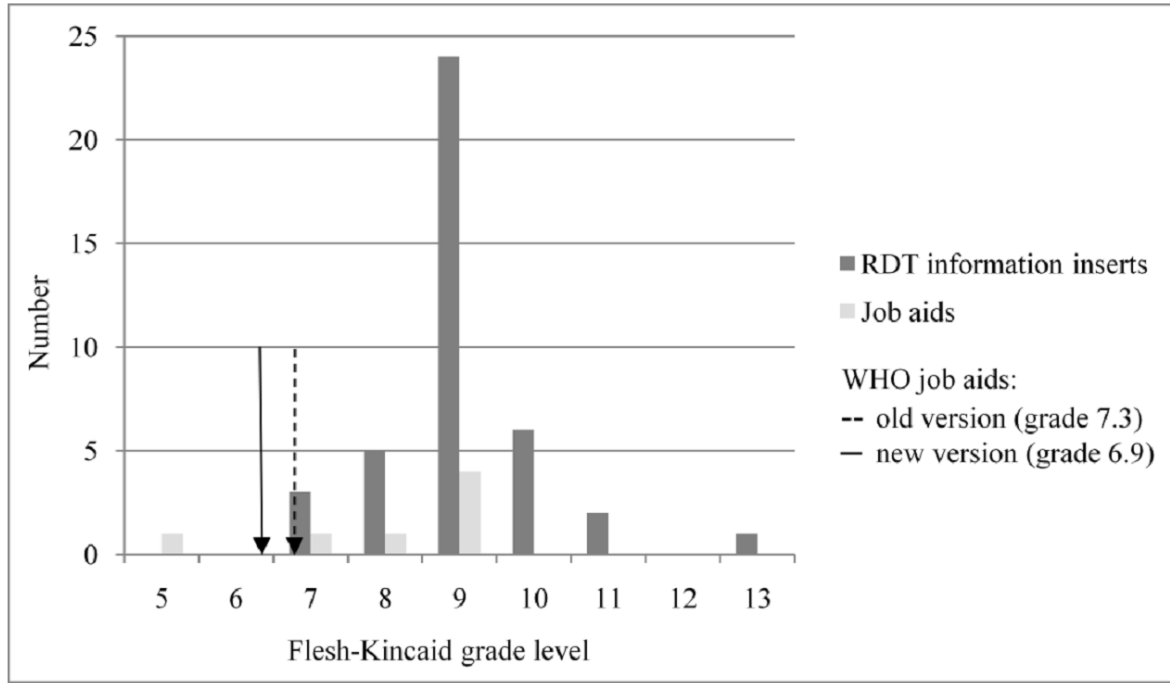

Figure 8: Readability of the RDT information inserts $(n=40)$ and job aids $(n=7)$ expressed as FleschKincaid grade level. 


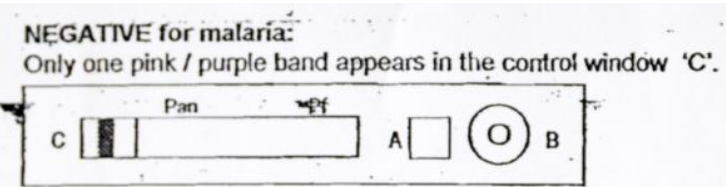

\section{POSITIVE for malaria:}

$P$. falciparum or mixed infection: In addition to the control band, two pink / purple bands appear at regions 'Pf' and 'Pan' in the test window T.

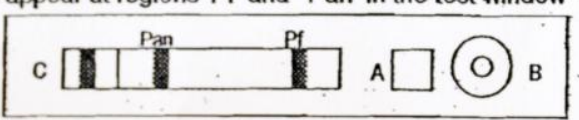

Other species (Non falciparum): In addition to the control band, one pink I purple band appears only at region 'Pan' in the test window 'T?.

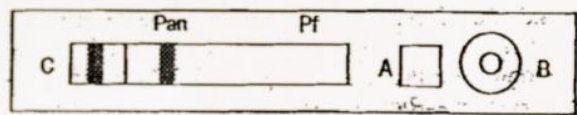

7. The test should be considered invalid if no bands appear on the device. Repeat the test with a new device ensuring that the test procedure has been followed accurately.

\section{LMMTATFONS OF THE FEST}

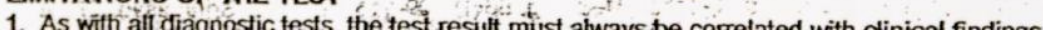

Figure 9: Interpretation section of the information insert of a three-band P. falciparum/pan-species RDT. For invalid tests, only the absence of all lines is mentioned, not the possibility of a visible test line without a control line. The invalid results are not depicted. The print quality is poor, no colours are used for the control and test lines.

Accuracy and relevance of information. The RDT kit's principle was described in all 40 information inserts. All but two inserts mentioned the materials provided in the RDT kit, and seven inserts provided a complete list of the materials required.

All RDT kit inserts mentioned the required specimen (in all cases both capillary and venous blood), all but one mentioned the anticoagulant to be used. Capillary blood sampling through finger prick was described in 35 inserts, of which one also added sampling by venipuncture. By contrast, the heel prick was not described in any information insert.

Biosafety precautions included the use of gloves (depicted or mentioned in 21 inserts, Figure 10) and safe waste disposal (addressed in 16 inserts), but 18 inserts did not mention any information on biosafety.

From Table 2 it is clear that a number of critical steps in RDT procedures were addressed by only part of the RDT inserts. Among them, there were relevant steps such as writing down sample identification, correct positioning of the transfer and buffer vial and the need for an adequate light source. 


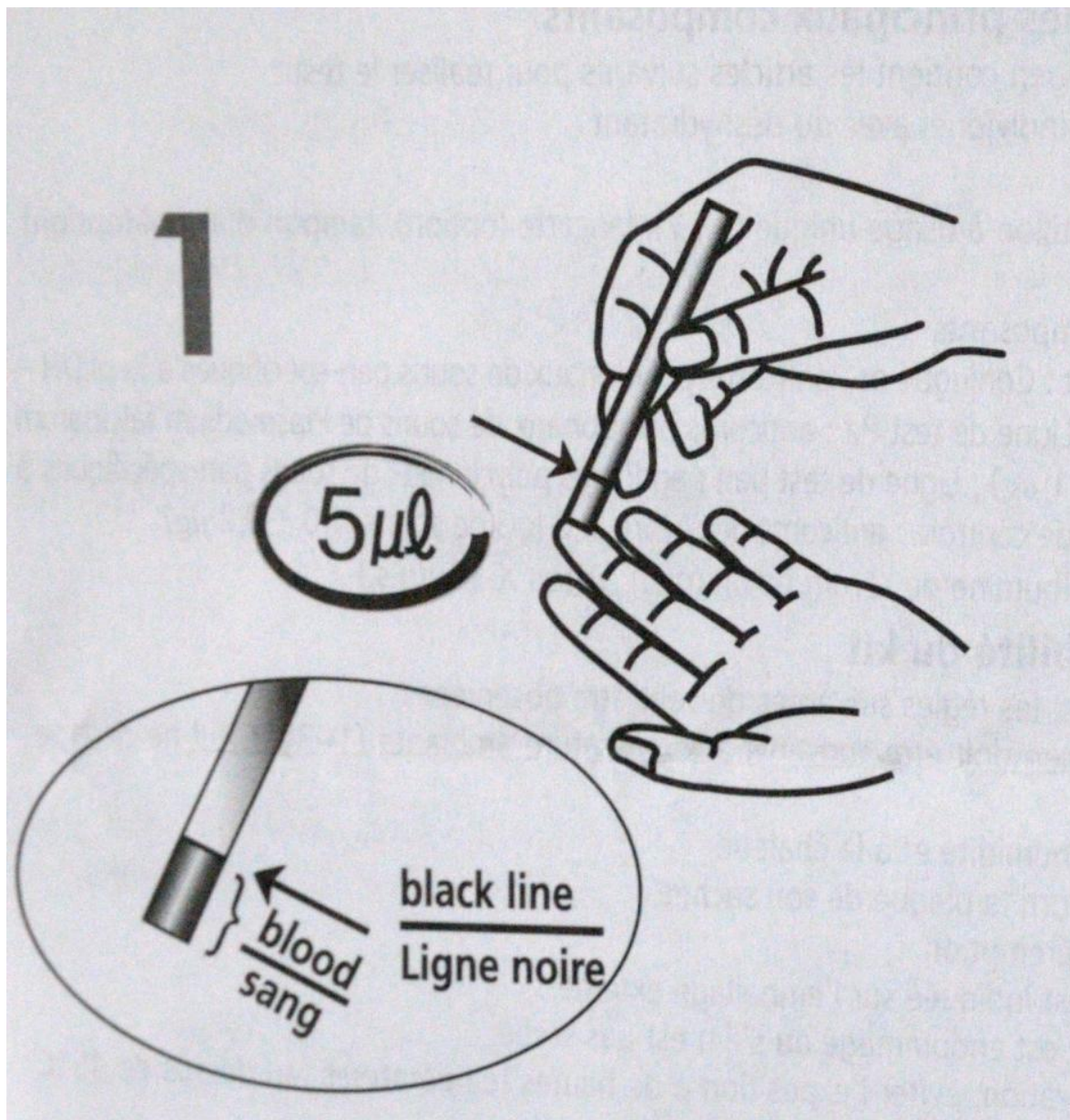

Figure 10: Illustration depicting sampling of capillary blood. The health care worker's hand is depicted without gloves. The simultaneous presence of English and French text may be difficult for a nonexperienced reader.

The complete array of all control and test line combinations was listed by only nine inserts. Fourteen inserts mentioned the absence of all lines as an invalid result but not the presence of a test line in the absence of a control line (Figure 9). In addition, errors in the interpretation of test lines were observed. For instance, the combination of a Pf-specific and a pan-specific test line was interpreted as $P$. falciparum without mentioning the possibility of a mixed infection (12/34 three- and four-band RDTs). Likewise, the combination of a Pv-specific and a pan-specific test line in case of a four-band RDT was interpreted as a Plasmodium vivax infection without mentioning the possibility of a mixed infection with Plasmodium ovale and Plasmodium malariae (6/7 RDTs). In addition, a visible pan-pLDH line was interpreted as a $P$. vivax infection (instead of non-falciparum species) in two inserts. 
Few inserts mentioned causes of false positive and false negative results (Table 2). One insert recommended to repeat the test in case of a negative RDT result and persistent suspicion of malaria, another warned about the prozone effect as a cause of a false negative result (Figure 4). RDT test results during treatment follow-up were addressed in 13 inserts, but the information listed in nine inserts was presented in a scattered way and only one insert clearly mentioned that HRP-2 persistence does not indicate a failed therapeutic response.

RDT test characteristics. Eight inserts did not provide information on sensitivity or specificity. Diagnostic characteristics were mostly expressed for P. falciparum and $P$. $\operatorname{vivax}(\mathrm{n}=31$ and $\mathrm{n}=22$ respectively), only one insert mentioned test characteristics for $P$. ovale and $P$. malariae. Sensitivity for $P$. falciparum was expressed by parasite density range in 10 inserts.

Bibliography cited in the information inserts. In total, 45 different references were used in the bibliography of the information inserts. One third of them referred to the original description of the target antigens, another 12 referred to general information on malaria and its diagnosis. Thirteen inserts cited evaluation studies of RDTs, but only three RDT kits referred to product-related studies. Two panels of identical references were shared by nine and eight inserts respectively.

RDT kits' names. Inconsistencies in the RDT kit names and referrals to target antigens were observed. For instance, four RDT kits had names referring to $P$. vivax although they used a pan-species Plasmodium antigen. Two other inserts did not mention the identity of the pan-species antigen (aldolase versus pan-pLDH). Five RDT kits from one manufacturer were supplied in identical boxes, carrying the same names and identical prints. Furthermore, there were slight but numerous differences between names as displayed on boxes versus those noted on device packages (eight had no brand name affixed), devices (15 differences) and information inserts (eight differences). Similar observations were made for buffer vials: five vials displayed only the manufacturer's name and one vial did not show brand nor manufacturer's name.

RDT kits' duplicates. During assessment of the RDT kits, apparent similarities between different RDT brands were observed. These similarities concerned, amongst others, design and shape of the device and content and layout of the information insert (e.g. numbers of samples used for calculation of test characteristics). In that way, six products were assumed to represent a common design and production platform for 16 different RDT brands.

Relation with CE marking and WHO listing. Overall, RDTs with evidence of GMP ( $\mathrm{n}=35)$ scored better compared to those without $(\mathrm{n}=5)$, although 
inadequacies, errors and omissions were observed between both groups. Three CE labelled RDT kits produced outside European Economic Area (EEA) had no ECREP indicated neither on the box nor in the information insert. For these kits, the CE symbol as displayed on the package had not the shape and relative dimensions of the 98/79/EC directive (Figure 11). Six of the eight RDT kits that did not mention data on sensitivity and specificity were CE labelled.

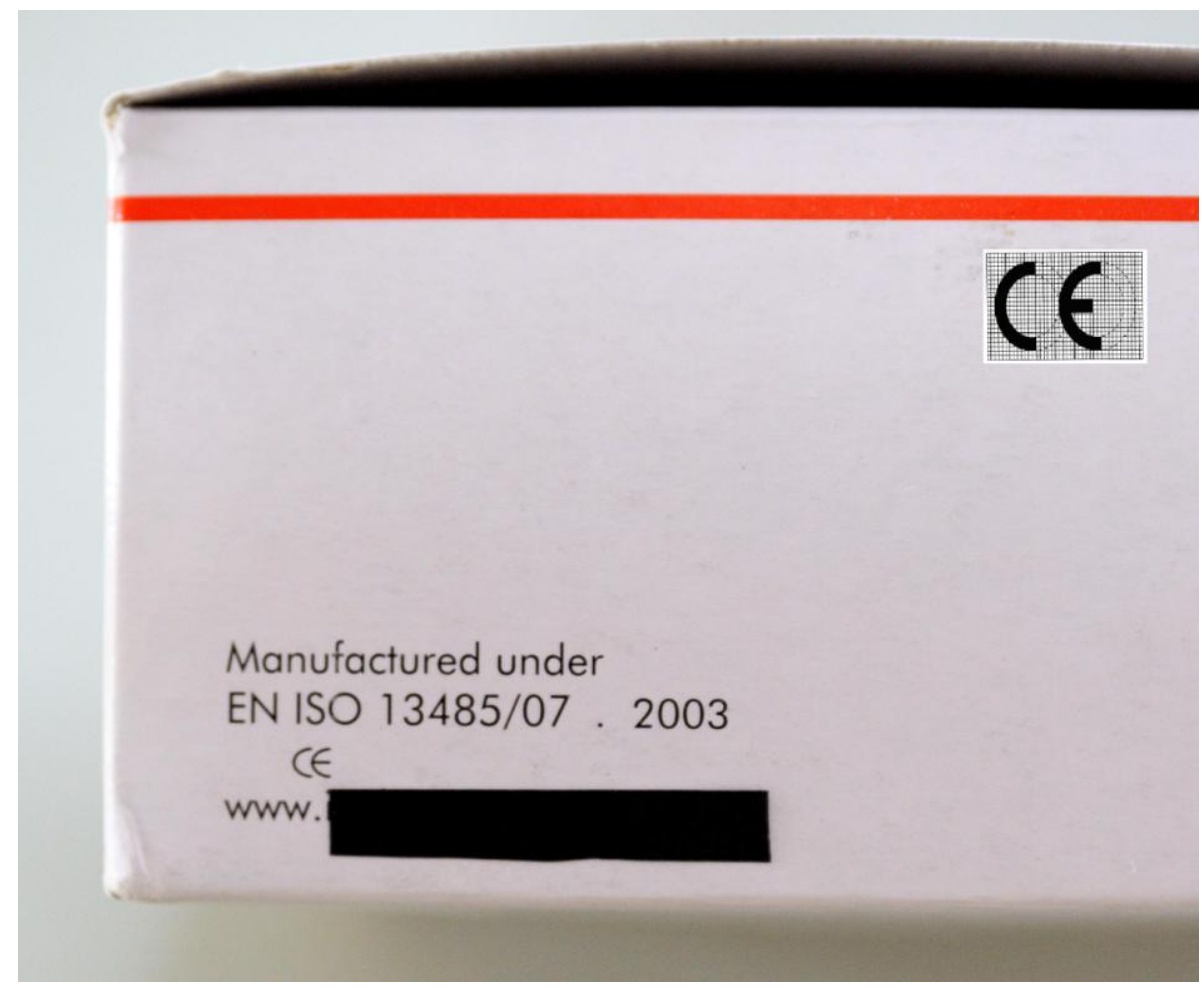

Figure 11: CE-label displayed on a RDT kit box. The shape and relative dimensions of the characters do not comply with the requirements as mentioned in the EC Directive 98/79 (depicted in the insert, upper right corner). There is no authorized representative (EC-REP) affixed, although required.

\section{Discussion}

Previous studies demonstrated that RDT manufacturers' instructions are insufficient to ensure accurate test performance by community health care workers, and welldesigned instructions such as the WHO generic job aids have proven to increase performance $[9,10,19,31]$. In practice however, such job aids still need to be adapted to the particular RDT brand used on site and, depending on the chains of supply, different RDT brands and versions may be available. For market exploration and choice of RDTs, laboratory managers will orient to RDT kits' names and labelling. Clear design and labelling of RDT kit components will contribute to correct storage and use; and laboratory staff will rely on RDT inserts for background information, adaptations of the generic procedures, interpretation and trouble-shooting. 
The design of the present study has its limitations. For instance, mainly RDTs marketed as cassettes were considered. However, cassettes are the mostly used platform and preferred by end-users over the strip format [9]. Besides, only a part of the marketed brands was evaluated; however, this evaluation studied about half of the 80 brands worldwide-marketed [2] including those frequently used in endemic and non-endemic settings.

With regard to the assessment of the information inserts, it should further be noted that the Flesch-Kincaid label (as discussed below) is only a proxy measure of readability. In addition, the layout of the inserts was assessed for typography but not for other features such as adequate use of headings, bullets, boldfacing, and amount of white space [32,33]. Finally, although RDTs were presently assessed against compiled criteria based on relevant documents, they were not evaluated by end-users in a real-life setting. On the other hand, as far as known, this is the first time that in vitro diagnostic medical devices (IVDs) were assessed for these characteristics.

\section{Problems in design and labelling of the RDT kits' components}

Despite these limitations, much information was generated on the quality and appropriateness of RDT packaging, labels and inserts. Apart from two exceptions (the plastic bags), boxes were well labelled, but shortcomings in labelling of device packages and buffer vials were more numerous. The absence of blood sampling and transfer system which was observed in nearly a quarter of RDT kits may create logistical problems when used in field settings. The problems in device design were of most concern. Characters indicating wells and reading labels that were embedded in the plastic cassette housings are difficult to distinguish and the simultaneous presence of two reading labels may cause confusion. Standardized and unequivocal characters or acronyms should be used for designating wells and reading labels and clear labelling with contrasting print should be ensured.

Some design issues were not compiled from previous studies but originated from ITM observations. Some of them may look trivial but have consequences in daily practice. For instance, device packages without pre-cut lids require scissors to open, which is neither safe nor practical in busy and remote settings. The space allocated for sample identification on most cassettes was large enough for writing down a sample number but not a patient name: this may meet the requirements in computerized settings but not those in a non-computerized field setting, where full names are written as recommended by WHO job aids instructions [16]. Likewise, it is evident that use of a felt pen for writing down sample identification is inappropriate for a field setting. Another example was the clockwise tightening of the buffer vial's cap for piercing the dropper bottle nozzle: ITM teams observed that laboratory staff unaware of this procedure simply cut off the distal end from the nozzle. The resulting opening tended to be very wide resulting in a too large volume of buffer added to each test and early emptying of the buffer vial. The RDT cassettes 
that remained without buffer were run with other buffers or with injection water, at the risk of causing false positive results [5].

\section{Readability level and typography of the information insert}

The Flesch-Kincaid grade level used in the present study has been demonstrated to be reliable and valid and is frequently used in health care issues such as consumer medical information (CMI) and patient education materials [27,30]. Its use in the present context should be interpreted with caution. Like any other reading formula, the Flesch-Kincaid readability tool assesses text structure but does not take into account the content. It further refers only to US grade levels and applies to English language. Other factors such as motivation and previous experience may influence comprehension, and linguistic and cultural issues may interfere [33]. Despite these limitations, it is of note that readability levels of all inserts exceeded the $6^{\text {th }}$ grade level, while the recommended level for health related information is equal or lower than this level $[27,30]$. The readability level of the job aids scored slightly better, but still higher compared to the WHO generic job aids. Too elevated reading levels have been consistently demonstrated in CMI documents such as those of home pregnancy tests, blood glucose monitoring and home blood pressure monitor equipment as well as in patient education brochures [25-27,32,34,35]. It should also be taken into account that end-users in endemic settings are likely to be non-native speakers of the language of the information insert (e.g. English, French, Portuguese), and may operate in stressful situations such as environmental disasters and war [36,37] which decrease actual reading levels [38]. Apart from the bad quality prints and small figures, the observed user-unfriendly typographic features may add to the decreased readability of the inserts, which were consistent with those documented for CMI materials $[25,30,32,34,35]$.

\section{Content of the information insert}

The lack of referral to biosafety procedures in nearly half of the inserts was striking and unacceptable, in particular because this is clearly mentioned in the WHO generic job aids [11]. Likewise were the differences between depicted and real devices and the use of non-realistic colours for depicting test lines which do not comply with WHO recommendations [16]. Numbers and sizes of illustrations did not comply with the established standards for patient education materials and CMI $[25,35,39]$.

The shortcomings in the RDT test interpretation session were in line with observations made during a recent external quality assessment on RDTs in a nonendemic setting. During that session, not reporting a mixed infection in case of the simultaneous presence of $P$. falciparum- and pan-specific test lines was demonstrated to be linked to the information inserts of the RDT kits used [18]. Although extremely rare in the experience at ITM, the presence of a visible test line 
in the absence of a control line points to invalid test results and should be added to the spectrum of possible line combinations, preferably with a picture. In addition, the persistence of HRP-2 after successful treatment and the production of pLDH by gametocytes should be clearly mentioned.

The poor description of diagnostic characteristics in the information inserts was another concern. Although current directives and recommendations do not specify details about origin, numbers and statistical validity of these test characteristics, manufacturers should be encouraged to provide as detailed and sound data as possible, including data on sensitivity in relation to parasite density and Plasmodium species. The low diagnostic sensitivity for $P$. malariae and $P$. ovale is well known [40]: few studies have included enough samples to provide reliable data for both species. Those that did mostly found a poor sensitivity, in particular for P. malariae [40-44]. In the absence of a thorough evaluation for both species, one could consider adding a statement mentioning the low overall diagnostic sensitivity for both species to the information insert, in order to avoid unrealistic expectations by the end-user relying on the pan-Plasmodium species nature of the targeted antigen [40].

The cited bibliography mainly referred to the original papers on the description of the antigens or general RDT evaluations. In addition to references addressing the RDT kit itself, references to one or more of the recent reviews on RDTs or WHO/FIND documents could be added, as they contain relevant information on the use and limitations of RDTs.

\section{Names and duplicates of RDTs, relation to GMP and CE labelling}

Among the inadequacies, erratic and inconsistent names were a frequent finding: they ranged from minor differences in RDT brand names as displayed on boxes, devices and their packages and information inserts to brand names suggesting $P$. vivax despite using a pan-pLDH target. In addition, shortcomings with regard to clear specification of target antigens were noted. For a laboratory manager finding his way among many other diagnostics and supplies, it is essential to get a quick and reliable idea about the intended use and the target antigens of RDTs. An unequivocal code for naming and short test descriptions should be considered, with mentioning of the (abbreviated) antigens as a requisite (e.g. Pf-pLDH, HRP-2 etc.).

With regard to the presumed RDT kit duplicates (kits presenting with similar presentation suggesting a shared design and production), it should be noted that WHO and FIND recognize this phenomenon [45]. WHO defines so-called "rebranded" products as products manufactured under identical conditions at the same manufacturing site as the original product, but labelled with a different product name and identifier. WHO encourages, in such case, joint application for the prequalification program or test evaluations [45-47]. The CE recognizes also the 
"re-branding" for commercial interests. The coexistence of multiple names for the same product however may create difficulties for instance in retrieving published information on test evaluations and may add to the complexity of post marketing surveillance, including traceability in case of batch recalls. To prevent these problems, the requirement of "re-branded" RDT kits to mention the original manufacturer should be considered. In addition, any RDT kit label (whether original or re-branded) should clearly distinguish the names of the manufacturer from that of the local distributor.

Shortcomings and errors were observed among CE-labelled and WHO-listed RDTs. Not affixing the EC-REP when required and not mentioning information on RDT test characteristics do not conform the 98/79/EC Directive [48]. It should further be noted that in case of malaria RDTs, the CE-label by itself is not a guarantee for intrinsic quality of performance. The 98/79/EC Directive includes the "Annex II", which lists diagnostics for which market release of any new lot has to be preceded by testing and approval by a competent authority, the so-called notified body. For diagnostics that are not listed in the "Annex II" (such as malaria RDTs), such testing and authorization are not required. Acquisition of the CE-label for these diagnostics is a purely administrative process, in which the manufacturer himself draws up the EC declaration of conformity. Unfortunately, the majority of laboratory and medical staff are unaware of this procedure. This can create a sense of "over-confidence" in CE-labelled products, based on the perception of quality associated with European labels. The inclusion of RDTs for malaria and other tropical diseases in the "Annex II" could represent a significant support for countries with weak regulatory overview.

\section{What can be done to improve the quality of RDT package and information inserts?}

Many shortcomings such as incomplete and incorrect labelling of boxes, device packages, cassettes and buffer vials can be easily remediated at minimal costs. Endusers and manufactures should reach consensus on uniform codes for labelling wells and reading windows and gradually reinforce the requirements for inserts and packaging. Generic recommendations as how to layout and how to appropriately design information inserts as well as use of figures can be found in the literature on CMI [25,35,39], RDT specific guidelines have been issued by the WHO [16]. Readability, cultural and linguistic backgrounds should be taken into account [23], and all texts and figures should be assessed for appropriateness and comprehension among the targeted end-users [10,49]. For the content of RDTs, the reference documents that were used to compile Tables 1 and 2 can give guidance. Examples of such checklists are added as additional files (Additional file 1 and Additional file 2). The information inserts should highlight key points in performance and interpretation in order to reduce the likelihood of potential errors. 
With regard to user-friendliness and adequacy of RDT presentation and instructions, interesting features not listed on the compiled criteria were noted. For instance, some device packages carried short instructions for use based upon the generic WHO job aids [16], witch may increase the test performance [9,10,19,31]. The availability of job aids for the different brands on the company websites in an adaptable text format may help Malaria National Programs to translate it in the enduser language and to adapt it on the local context. In addition, some RDT kits provided a glossary with explanation of the affixed symbols, which may help in their comprehension and acquisition and another five had all essential information printed on a single (lateral) side of the box, contributing to easy storage (Figure 12). Another asset was the presence of more than one buffer vial per RDT box, as shortage and replacement of buffer vials is a common problem in resource limited settings [5]. Likewise, the above described observations and corrective measures could be extended to other IVDs, such as human immunodeficiency virus RDTs.

Of course, it should be noted that adequate packages and information inserts by themselves are not a guarantee for competent use of RDTs. Simply distributing the RDTs and instructions does not work, and RDT instructions on their own will not change professional behaviours [20]. Thorough training and performance monitoring are needed for correct performance [16].

\section{Conclusion}

In conclusion, malaria RDTs showed shortcomings with regard to quality of construction, design and labelling of boxes, device packages, devices and buffers. Information inserts were difficult to read and lacked relevant information. Particular problems were observed in the consistency and appropriateness of RDT brand names and in the referral to the antigens used. In general, CE-labelled and WHOlisted RDTs scored better compared to those without but inadequacies were observed among these RDTs. Addressing the quality of RTD package and information inserts in evaluation programs such as the WHO/FIND products testing program could stimulate the manufacturers to remediate these shortcomings. Likewise, inclusion of malaria RDTs in the "Annex" II of the 98/79/EC directive might represent a powerful support from the European Community towards the quality of in-vitro diagnostics in tropical countries. 


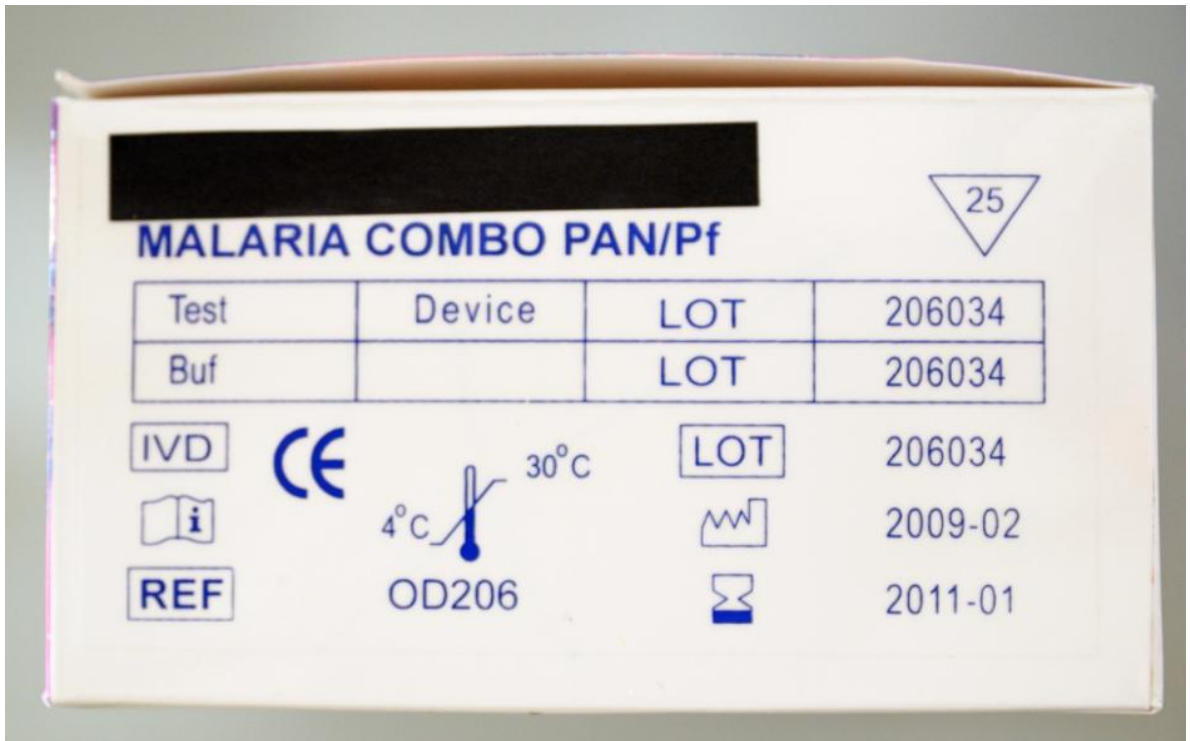

Figure 12: Example of the lateral side of a RDT box: all essential information is printed on a single side of the box, contributing to clear storage. Of note, the symbol used for "sufficient for" is not used a required by EN 980: the inverted triangle should contain the " $\sum$ " character and affix the number below of beneath.

\section{List of abbreviations}

CE: Conformité Européenne; CMI: Consumer medical information; EC-REP : European Authorized Representative; EEA: European Economic Area; FDA: Food and drug administration; FIND: Foundation for Innovative New Diagnostics; FHML: Faculty of Health, Medicine and Life Sciences; GMP: Good manufacturing practice; HRP-2: Histidine-rich protein 2; ISO: International organization for standardization; ITM: Institute of Tropical Medicine; IVDs: in vitro diagnostic medical devices; P: Plasmodium; pan-pLDH: pan Plasmodium-specific parasite lactate dehydrogenase; Pf-pLDH: Plasmodium falciparum-specific parasite lactate dehydrogenase; pLDH: parasite lactate dehydrogenase; Pv-pLDH: Plasmodium vivax-specific parasite lactate dehydrogenase; RDT(s): Rapid diagnostic test(s); WHO: World Health Organization.

\section{Acknowledgements}

We would like to thank Peter Verbruggen, Sint Lucas Antwerpen, Karel de GroteHogeschool, Antwerp, Belgium for advices about assessing typography and Dionicia Gamboa, Institute of Tropical Medicine "Alexander Von Humboldt", Lima, Peru for fruitful discussions and suggestions. 


\section{References}

1. World Health Organization: Guidelines for the treatment of malaria, second edition, 2010. [http://whqlibdoc.who.int/publications/2010/9789241547925 eng.pdf]

2. Bell D, Perkins MD: Making malaria testing relevant: beyond test purchase. Trans $R$ Soc Trop Med Hyg 2008, 102: 1064-1066.

3. Chiodini PL, Bowers K, Jorgensen P, Barnwell JW, Grady KK, Luchavez J, Moody AH, Cenizal A, Bell D: The heat stability of Plasmodium lactate dehydrogenase-based and histidine-rich protein 2-based malaria rapid diagnostic tests. Trans $R$ Soc Trop Med Hyg 2007, 101: 331-337.

4. Gillet P, Mori M, Van Esbroeck M, Van Den Ende J, Jacobs J: Assessment of the prozone effect in malaria rapid diagnostic tests. Malar J 2009, 8: 271.

5. Gillet $\mathrm{P}$, Mori M, Van Den Ende J, Jacobs J: Buffer substitution in malaria rapid diagnostic tests causes false-positive results. Malar J 2010, 9: 215 .

6. Jorgensen P, Chanthap L, Rebueno A, Tsuyuoka R, Bell D: Malaria rapid diagnostic tests in tropical climates: the need for a cool chain. Am J Trop Med Hyg 2006, 74: 750-754.

7. McMorrow ML, Masanja MI, Abdulla SM, Kahigwa E, Kachur SP: Challenges in routine implementation and quality control of rapid diagnostic tests for malaria--Rufiji District, Tanzania. Am J Trop Med Hyg 2008, 79: 385-390.

8. World Health Organization: Lot-testing: Using quality control parasite dilutions to test the sensitivity of malaria RDTs. 2010.

[http://www.wpro.who.int/sites/rdt/using rdts/qa/lot testing.htm]

9. Rennie, W.: Developing and testing a generic job aid for malaria rapid diagnostic tests (RDTs). 2004.

http://www.wpro.who.int/internet/resources.ashx/RDT/docs/pdf_version/Developing_and_testing_an_RDT_Job_Ai d.pdf]

10. Rennie W, Phetsouvanh R, Lupisan S, Vanisaveth V, Hongvanthong B, Phompida S, Alday P, Fulache M, Lumagui R, Jorgensen P, Bell D, Harvey S: Minimising human error in malaria rapid diagnosis: clarity of written instructions and health worker performance. Trans $R$ Soc Trop Med Hyg 2007, 101: 9-18.

11. World Health Organization: How to use a Rapid Diagnostic Test (RDT): A guide for training at a village and clinic level. 2008.

[http://www.wpro.who.int/sites/rdt/using_rdts/training/rdt_training_falciparum.htm]

12. World Health Organization: List of known commercially-available antigen-detecting malaria RDTs with adequate evidence of good manufacturing practice. 2009. [http://www.wpro.who.int/internet/resources.ashx/RDT/docs/MD_table34+(1)_totallistofISO1 31485criteria.pdf]

13. Maltha J, Gillet P, Cnops L, Van Den Ende J, Van Esbroeck M, Jacobs J: Malaria rapid diagnostic tests: Plasmodium falciparum infections with high parasite densities may generate false positive Plasmodium vivax pLDH lines. Malar J 2010, 9: 198.

14. World Health Organization: Hepatitis B Assays: Operational Characteristics Report 1 \& 2. 2004. [http://whqlibdoc.who.int/publications/2004/9241592206.pdf]

15. World Health Organization: The Use of Rapid Malaria Diagnostic Tests, Second Edition. 2006. [http://www.wpro.who.int/NR/rdonlyres/A30D47E1-1612-4674-8DF8FCA031CDB9BA/0/Reduced web2_MalariaRDT_20062ndedition.pdf]

16. World Health Organization: Notes on job aids for malaria rapid diagnostic tests (RDTs). 2006. [http://www.wpro.who.int/NR/rdonlyres/06B185E0-7E63-4991-B8F5B3D62D6C8373/0/SHORTNOTESONJOBAIDfinal.pdf]

17. World Health Organization: HIV Rapid Assays: Operational Characteristics Report 16. 2009. [http://www.who.int/entity/diagnostics laboratory/publications/Report16 final.pdf]

18. Gillet P, Mukadi P, Vernelen K, Van Esbroeck M, Muyembe J-J, Bruggeman C, Jacobs J: External quality assessment on the use of malaria rapid diagnostic tests in a non-endemic setting. Malar J 2010, 9: 359 .

19. Harvey SA, Jennings L, Chinyama M, Masaninga F, Mulholland K, Bell DR: Improving community health worker use of malaria rapid diagnostic tests in Zambia: package instructions, job aid and job aid-plus-training. Malar J 2008, 7: 160.

20. Knebel, E.: The Use of Manual Job Aids by Health Care Providers: What Do We Know? 2000. [www.qaproject.org/pubs/PDFs/ISSUESJA.PDF]

21. Moonasar D, Goga AE, Kruger PS, La Cock C, Maharaj R, Frean J, Chandramohan D: Field evaluation of a malaria rapid diagnostic test (ICT Pf). $S$ Afr Med J 2009, 99: 810-813. 
22. Murray CK, Bell D, Gasser RA, Wongsrichanalai C: Rapid diagnostic testing for malaria. Trop Med Int Health 2003, 8: 876-883.

23. Seidahmed OM, Mohamedein MM, Elsir AA, Ali FT, Malik eF, Ahmed ES: End-user errors in applying two malaria rapid diagnostic tests in a remote area of Sudan. Trop Med Int Health 2008, 13: 406-409.

24. Tavrow P, Shabahang J, Makama S: Vendor-to-vendor education to improve malaria treatment by private drug outlets in Bungoma District, Kenya. Malar J 2003, 2: 10.

25. Wallace LS, Keenum AJ, Roskos SE, Blake GH, Colwell ST, Weiss BD: Suitability and readability of consumer medical information accompanying prescription medication samples. Patient Educ Couns 2008, 70: 420-425.

26. Wallace LS, Zite NB, Homewood VJ: Making sense of home pregnancy test instructions. $J$ Womens Health (Larchmt) 2009, 18: 363-368.

27. Wallace LS, Keenum AJ, Roskos SE, Koopman RJ, Young KG: Blood glucose monitor quick reference guides: are they suitable for patients? Diabetes Technol Ther 2008, 10: 1115.

28. Tests Document Readability. [http://www.online-utility.org/english/readability_test_and_improve.jsp]

29. Flesch R: How to Write Plain English. 2004.

[http://www.mang.canterbury.ac.nz/writing_guide/writing/flesch.shtml]

30. Wallace LS, Roskos SE, Weiss BD: Readability characteristics of consumer medication information for asthma inhalation devices. $J$ Asthma 2006, 43: 375-378.

31. Mayxay M, Newton PN, Yeung S, Pongvongsa T, Phompida S, Phetsouvanh R, White NJ: Short communication: An assessment of the use of malaria rapid tests by village health volunteers in rural Laos. Trop Med Int Health 2004, 9: 325-329.

32. Arnold CL, Davis TC, Frempong JO, Humiston SG, Bocchini A, Kennen EM, Lloyd-Puryear M: Assessment of newborn screening parent education materials. Pediatrics 2006, 117: S320-S325.

33. White LJ, Jones JS, Felton CW, Pool LC: Informed consent for medical research: common discrepancies and readability. Acad Emerg Med 1996, 3: 745-750.

34. Wallace LS, Keenum AJ: Using a home blood pressure monitor: do accompanying instructional materials meet low literacy guidelines? Blood Press Monit 2008, 13: 219-223.

35. Wallace LS, Keenum AJ, DeVoe JE: Evaluation of consumer medical information and oral liquid measuring devices accompanying pediatric prescriptions. Acad Pediatr 2010, 10: 224-227.

36. Hashizume M, Kondo H, Murakami T, Kodama M, Nakahara S, Lucas ME, Wakai S: Use of rapid diagnostic tests for malaria in an emergency situation after the flood disaster in Mozambique. Public Health 2006, 120: 444-447.

37. Hawkes M, Katsuva JP, Masumbuko CK: Use and limitations of malaria rapid diagnostic testing by community health workers in war-torn Democratic Republic of Congo. Malar J 2009, 8: 308 .

38. Doak LG, Doak CC, Meade CD: Strategies to improve cancer education materials. Oncol Nurs Forum 1996, 23: 1305-1312.

39. Kaphingst KA, DeJong W: The educational potential of direct-to-consumer prescription drug advertising. Health Aff (Millwood) 2004, 23: 143-150.

40. Grobusch MP, Hanscheid T, Zoller T, Jelinek T, Burchard GD: Rapid immunochromatographic malarial antigen detection unreliable for detecting Plasmodium malariae and Plasmodium ovale. Eur J Clin Microbiol Infect Dis 2002, 21: 818-820.

41. Maltha J, Gillet P, Bottieau E, Cnops L, Van Esbroeck M, Jacobs J: Evaluation of a rapid diagnostic test (CareStart Malaria HRP-2/pLDH (Pf/pan) Combo Test) for the diagnosis of malaria in a reference setting. Malar J 2010, 9: 171.

42. Van der Palen M, Gillet P, Bottieau E, Cnops L, Van Esbroeck M, Jacobs J: Test characteristics of two rapid antigen detection tests (SD FK50 and SD FK60) for the diagnosis of malaria in returned travellers. Malar J 2009, 8: 90.

43. van Dijk DP, Gillet P, Vlieghe E, Cnops L, Van Esbroeck M, Jacobs J: Evaluation of the Palutop+4 malaria rapid diagnostic test in a non-endemic setting. Malar J 2009, 8: 293.

44. van Dijk DP, Gillet P, Vlieghe E, Cnops L, Van Esbroeck M, Jacobs J: Evaluation of the Immunoquick+4 malaria rapid diagnostic test in a non-endemic setting. Eur $J$ Clin Microbiol Infect Dis 2010, 29: 577-583. 
45. World Health Organization: Overview of the prequalification of diagnostics assessment process. 2010.

[http://www.who.int/entity/diagnostics laboratory/evaluations/pqdx 007 pq_overview docu ment v3.pdf]

46. World Health Organization: Malaria Rapid Diagnostic Test Performance; Results of WHO product testing of malaria RDTs: Round 1 (2008). 2009.

[http://www.finddiagnostics.org/resource-centre/reports brochures/malaria-diagnostics-report2009.html]

47. World Health Organization: Malaria Rapid Diagnostic Test Performance; Results of WHO product testing of malaria RDTs: Round 2 (2009). 2010.

[http://www.finddiagnostics.org/resource-centre/reports_brochures/malaria-diagnostic-testreport-round2.html]

48. European Commission: European standards in vitro diagnostic medical devices. 1998.

[http://eur-lex.europa.eu/LexUriServ/LexUriServ.do?uri=OJ:L:1998:331:0001:0037:EN:PDF]

49. Taylor HA: Barriers to informed consent. Semin Oncol Nurs 1999, 15: 89-95. 
Additional file 1: Example of operational checklist for packaging, labelling and instructions of RDTs.

\section{Items to be checked}

\section{Box: construction and design}

Is the box construction humidity-resistant?

Are the labels on the box humidity-resistant?

Are names used on device packaging, device, buffer and information insert identical?

\section{Box: information displayed}

Is the EC-REP mentioned on CE labelled RDTs when required?

Is there a reference to the intended use in the RDT kit's name or in additional information?

Is there correct reference to the targeted species ( $P$. vivax, non-falciparum species) in the RDT kit's name?

Is there a list of the RDT kit's components included in the box displayed?

Is all essential information present: expiry date, numbers of tests included, storage conditions?

\section{Kit contents:}

Is a capillary blood sampling system (lancet and alcohol swab) included?

Is a blood transfer system (capillary, pipette or tube) included?

\section{Device package and content: construction and design}

Is the material of the device package humidity-resistant?

Is it a desiccant with saturation indicator?

\section{Device package and content: information displayed}

Is essential information present: expiry date, lot number, test kit name?

Is there a warning label "do not swallow" on the desiccant?

\section{Device: construction and design}

Is the space for sample identification large enough for writing sample identification and writable with standard pen?

Is the complete RDT name write on the device?

Is it a single reading label consisting of acronyms referred to the target (e.g. "Pf", "Pan")?

\section{Buffer vial: construction and design}

Is the buffer vial leak proof?

Is the label well fixed to the vial and humidity-resistant?

\section{Buffe vialr: information displayed}

Is essential information present: expiry date, lot number, storage conditions, correct RDT kit's name?

Does the package insert instructions mention on how to pierce the buffer vial dropper?

\section{Package insert: information}

Are date of release and version number present?

\section{Package insert: content}

Is the identity of target antigens clearly mentioned?

Is there referral to biosafety precautions (gloves, safe waste disposal, etc.)?

Do the illustrations of the devices match with the real device?

Are realistic colours used for the illustrations (e.g. control and test lines depicted as red/purple)?

Are data on test characteristics presented (sensitivity, specificity)? 
Additional file 2: Example of a checklist for the content of the information inserts.

\section{Items addressed in procedure section}

Bring the RDT device and buffer to room temperature

Check the integrity of the device package

Check expiry date

Use the device immediately after opening

Place the device on a level surface

Check the desiccant for signs of exposure to humidity

Write down sample identification

Wipe finger with alcohol

Allow the finger to dry before pricking

Hold the transfer device (loop, straw) vertical

Hold the buffer vial vertical

Do not to use another buffer than the one provided with the kit

Use an adequate light source for reading

\section{Items addressed in interpretation section}

All possible line combinations for invalid test results are mentioned

All possible test line combinations for positive test results are mentioned

Interpretation of a faint test line as positive is mentioned

Causes of false negative results are mentioned, in particular low parasite densities

Causes of false positive results are mentioned, e.g. presence of the rheumatoid factor

Persistence of HRP-2 is mentioned

To repeat the test in case of a negative RDT result and persistent suspicion of malaria is mentioned 


\section{Chapter VII}

\section{Summary, general discussion and future perspectives}




\section{Summary, general discussion and future perspectives}

The present thesis aimed to close some gaps in knowledge about the test characteristics of malaria RDTs, end-user performance and the quality of the malaria RDT kits' packages and information inserts.

In Chapter II, the test characteristics of two one step malaria RDTs targeting $P$. vivax were assessed: SD FK70 malaria $\mathrm{Ag} \mathrm{Pv}$ (only $P$. vivax detection) en SD FK80 Pf/ Pv (P. falciparum and P.vivax detection). Although the sensitivities of both malaria RDTs for the detection of $P$. vivax withstand comparison with the best among other malaria RDTs, there were some concerns. First, the sensitivity for the diagnosis of $P$. vivax is still not high enough to exclude the diagnosis of $P$. vivax in a reliable way. Further, it was noted - as for other malaria RDT brands - that sensitivity declines at parasite densities below 5,000/ $\mu$ l and particularly below $500 / \mu 1$. Moreover, the sensitivities for the diagnosis of P. falciparum (for SD FK80) were lower compared to those obtained for other malaria RDT brands, including malaria RDTs using identical antibodies produced by the same manufacturer. Finally, the apparent cross-reactions of $P$. falciparum with the $P$. vivax test line are a serious limitation as to the applicability of these malaria RDTs in a field setting, in particular the two-band SD FK70. On the positive side, the clear and unequivocal distinction between $P$. vivax and $P$. ovale samples proved to be helpful in the reference diagnosis; SD FK70 is currently used in CLKB as a back-up tool for the microscopic diagnosis of both species pending species-specific PCR.

Chapter III assessed the occurrence and the intensity of the prozone effect in a laboratory and in a field setting. HRP-2 RDTs but not Pf-pLDH RDTs were demonstrated to be affected by prozone, although at a different extent and with lotto-lot variations. Prozone was found to be rare in terms of intensity and frequency: negative and faint test lines accounted for a minority of prozone-positive samples (18.2\% of 124 prozone positive samples) and the most affected brand among the malaria RDT brands assessed showed prozone with faint or negative test lines in $1.2 \%$ of all Plasmodium positive samples. However, faint test lines and even weak test lines tend to be disregarded as negative by end-users in endemic and nonendemic settings. Also the deployment of malaria RDTs to the community setting where laboratory facilities for dilution of samples are not available - is of further concern [1-3]. In addition, the proportion of samples with elevated parasite densities will depend on local factors such as malaria endemicity and background immunity. The present findings should be taken into account when making the choice between either Pf-pLDH or HRP-2 based RDTs.

Chapter IV describes false-positive results in malaria RDTs caused by buffer substitution. About three quarters of malaria RDTs assessed showed a false-positive test line with at least one sample and one substitute buffer. Although no data on the use of substitute buffers exist, we observed many of such buffer replacements in the field and were also addressed by alumni colleagues about this problem. The results of this study might contribute to further adaptations in the design of malaria RDT kits, such as adding more than one buffer vial to the malaria RDT kits and adding a 
warning to the information insert mentioning to stick to the dedicated buffer of the RDT kit.

In Chapter $\mathbf{V}$ the results of an external quality assessment session on malaria RDTs in a non-endemic setting are reported. The results indicated an excellent analytical performance of the malaria RDTs but pointed also to small albeit consistent errors in the test results interpretations, part of which were caused by errors in the malaria RDT kits' information inserts. The answers to the questionnaire confirmed the low exposure of the laboratory staff to Plasmodium positive samples but most importantly revealed a reliance on malaria RDTs as the primary and single tool for the diagnosis of malaria outside opening hours of the laboratory. In view of the possible diagnostic errors and the technical limitations of malaria RDTs, this practice should be avoided.

In Chapter VI, malaria RDTs were assessed for adequate and correct package, design and labelling of boxes and components as well as for readability and accuracy of information. Numerous shortcomings were noted, also among CElabelled, ISO13485 certified products and products included in the WHO/FIND procurement list. Most relevant shortcomings were ambiguous labelling of the reading scales and deficient labelling of the buffer vials. The readability level of the information inserts was too elevated and the typography was user-unfriendly. Essential topics such as measures of biosafety were frequently not addressed and incomplete or incorrect information was observed with regard to depicted devices and interpretation of test lines. However, most of these errors may be easily corrected and clear instructions from professional and regulatory authorities might improve the quality of RDT packages and information inserts at a limited additional cost. Leading authorities such as the WHO or the European Community could stimulate such corrections and consolidate them, for instance as part of prequalification requirements or by adding to the so-called "Annex II products" for which the EU directive on in vitro diagnostics (IVD) prescribes lot testing prior to market release [4-7].

From the present studies it is clear that RDTs represent an essential part of malaria diagnosis, both in endemic and non-endemic settings. Relevant conclusions emerging from the above described chapters address the analytical, pre- and postanalytical characteristics of malaria RDTs. As part of the analytical characteristics, we demonstrated the diagnostic characteristics of two one-step RDTs targeting $P$. vivax and studied technical factors linked to the design of RDTs including the prozone effect and the false-positive results after buffer substitution. Both factors are in part related to the design of immunochromatographic tests, with no washing step to remove blocking antigens or non-specific interactions. Pre-analytical and postanalytical conclusions were revealed by the external quality assessment session of the malaria RDTs: they included errors with regard to (i) indications and diagnostic strategy (pre-analysis) and (ii) interpretation and reporting (post-analysis). Such errors may swiftly be corrected by simple measures and procedures which could be incited by working documents issued by professional organizations, As the errors in interpretation and reporting were related to the manufacturer's instructions, we further explored RTD packages and information inserts and pointed to a number of shortcomings, which may be easily corrected at a reasonable cost. 
In the present study we also identified tracks and additional research questions. Chapters II and III cited the study of test characteristics of Pf-pLDH based RDTs. Of those, the SD FK40 (Standard Diagnostics Inc., Hagal-Dong, Korea), which is a PfpLDH/pan-pLDH based RDT, has been evaluated in a laboratory setting [8]. Evaluations of CareStart Pf-pLDH/pan-pLDH (Access Bio Inc, Monmouth Junction, NJ, USA) and SD FK90 (Standard Diagnostics Inc., Hagal-Dong, Korea), a combined HRP-2 and Pf-pLDH malaria RDT, are ongoing. The latter RDT would be - provided excellent test characteristics at a reasonable price - a good candidate for field settings: it is known that HRP-2 can persist for weeks after treatment of $P$. falciparum infection [9]. The combined detection of HRP-2 and Pf-pLDH by a RDT such as SD FK90 would not only counter the prozone effect, but also enable distinction between recent or treated $P$. falciparum infections. In addition, in Chapter II we observed occasional cross reactions of $P$. falciparum with the $P$. vivax-specific Pv-pLDH, reason why we decided to explore this phenomenon: we challenged a panel of $P$. vivax-detecting RDTs with a set of $P$. falciparum samples with high parasite densities. False positive Pv-pLDH lines were observed in 6/9 RDTs at frequencies ranging from $8.2 \%$ to $29.1 \%$ of 85 samples tested [10]. The readability problems of the package inserts of RDTs described in Chapter VI raised questions about interpretation and knowledge of diagnostic symbols developed by ISO 15223-1:2007 and EN 980:2008, which are used to circumvent the problem of the different languages of potential end-users. Together with our colleagues in the South, we conducted a survey among health care professionals in the Democratic Republic of the Congo, Cuba, Cambodia and Belgium in order to assess their actual and contextual knowledge of diagnostic and hazard symbols. For all participants combined, knowledge of symbols proved to be poor, with only three out of 20 symbols meeting the $67 \%$ correct score (ISO 3864 criteria). The two hazard symbols were familiar to most of the participants, but scored poor $(<50 \%)$. The poor comprehension of the symbols clearly evidenced the need of targeted training for a correct application of RDTs and in-vitro diagnostics in general, and manufacturers should include in the information inserts glossaries explaining the meaning of the symbols [11].

Future research will focus on the use of RDTs in resource-poor malaria endemic settings. In these settings, external quality assessments of RDTs such as performed in Chapter $\mathrm{V}$ require alternative test specimens resistant to harsh tropical climate conditions. Recently, dried tube specimens (DTS) have been developed as a simple and stable vehicle for serum samples in proficiency testing of HIV-antibody detection [12]. A similar system will be developed for blood specimens infected with Plasmodium species; it will be validated together with our partners in Lima, Peru.

Observations made in Chapter II and in other RDT evaluations performed at CLKB indicated that up to one third of positive test lines showed faint and weak intensities [8,13-17]. The problem of disregarding such low line intensities as negative might be higher than expected. Indeed, presbyopia (an accommodation deficit resulting in near vision failure) often goes unrecognised. In native Africans, its prevalence has been reported to be higher as compared to Europeans and its onset apparently occurs at lower ages [18,19] A role of presbyopia as an error in reading RDTs has been hypothesised [20], but the actual extent of the problem however is not known. Therefore, in collaboration with our partners in Kinshasa, a study measuring the 
prevalence of presbyopia among end-users is presently set-up in the Democratic Republic of the Congo.

Observations made in Chapter VI about shortcoming in packaging, labelling and information of malaria rapid diagnostic tests will need a follow-up and should be expanded to other IVD. This work will be realized though the QUAMED network. The QUAMED network - hosted by ITM - brings together operational and academic organizations from the North and the South with as objective to contribute to build universal access to quality medicines.

The results of the studies conducted as part of this thesis's well as those of other publications in the field of laboratory aspects of RDTs are synthesized in two concluding tables. They have been compiled and published as part of the didactic report addressed to the participants of the external quality assessment session described in Chapter V [21]. Table 1 lists the added value of malaria RDTs in the laboratory diagnosis of malaria in a non endemic setting, with the strengths, limitations and particularities of the different antigens and Plasmodium species. Table $2 \mathrm{a}$ and $2 \mathrm{~b}$ lists the "do's and don'ts" to be respected in the daily laboratory use of RDTs.

It can be concluded that RDTs are of clear benefit in malaria diagnosis. Sound knowledge of their characteristics and limitations by the end-user will increase their value as a diagnostic tool. 
Table 1: How can malaria rapid diagnostic tests add to the diagnosis of malaria in a non-endemic setting?

\begin{tabular}{lll}
\hline $\begin{array}{l}\text { Requirements for malaria } \\
\text { diagnosis }\end{array}$ & $\begin{array}{l}\text { Contribution of malaria } \\
\text { RDTs }\end{array}$ & Comments \\
\hline
\end{tabular}

Excellent sensitivity, especially for $P$. falciparum $>100$ parasites $/ \mu 1$

Considerably helpful in the diagnosis of malaria

Do not rule out malaria in a confident way (microscopy needed as well)

Timely confirmation or exclusion of the diagnosis of malaria with prompt referral in case of doubt

Distinction between $P$. falciparum (possible lifethreatening) and the nonfalciparum species

Assessment of parasite densities, in particular recognition of critical values (>2\% of red blood cells infected)

\section{Recognition of $P$. falciparum stages and hemozoin}

Of considerable help in the identification of $P$.

falciparum

Of no help
False-negatives for $P$. falciparum at low parasite densities $(<100 / \mu 1)$, occasionally above

Certain HPR-2 mutations may not be picked-up

Prozone effect is rare but occurs, particularly in HRP-2 based malaria RDTs

Only of moderate help for diagnosis of $P$. vivax and of little help for $P$. ovale and $P$. malariae

Mixed infections are rare but not excluded if $P$. falciparum- and panspecies antigen lines are present

Line intensities are indicative for parasite density but here is a very large overlap

Unique HRP-2 line may point to low parasite density 
Table 2a: Do's and don'ts of malaria diagnostic tests (endemic and non-endemic settings).

\begin{tabular}{l} 
DO \\
\hline Check the control line - repeat \\
malaria RDT if control line is not \\
visible
\end{tabular}

Respect the correct volume (you may use a pipette instead of the transfer device)

Consider a faint line also as a positive line

Repeat a negative malaria RDT in case of suspicion of malaria

\section{Comments}

Absence of control line means invalid test and no conclusion can be drawn

Too little blood may cause false-negative results

Too much blood will cause decreased clearance of the strip, hindering reading

Too much blood may increase the risk/intensity of prozone effect

Any visible line is a positive line

Repeat after 8-12 hours for a successive 4 times over 36 hours to rule out malaria

\section{DON'T}

Do not store malaria RDTs in the freezer

Do not read before or beyond the recommended reading time

Do not use malaria RDTs for treatment follow-up

\section{Comments}

Freezing will destroy colloidal gold

Reading to early may cause false-negative results

Waiting too long may cause false-positive results

HRP-2 based malaria RDTs remain positive for weeks after P. falciparum infection, gametocytes express $\mathrm{pLDH}$ and aldolase

Table 2b: Do's and don'ts of malaria diagnostic tests (addendum for non-endemic settings).

\begin{tabular}{l}
\hline DO \\
\hline $\begin{array}{l}\text { Always combine malaria RDT } \\
\text { with microscopy }\end{array}$ \\
\hline
\end{tabular}

Use a transfer pipette

\section{Comments}

See Table 1

Transfer devices of malaria +RDT kits tend to be small and somewhat difficult to manipulate

For Belgium, see request form and instructions on: https://www.iph.fgov.be/epidemio/epinl/plabnl/N_Plasmodium.pdf https://www.iph.fgov.be/epidemio/epifr/plabfr/F Plasmodium.pdf
Send any positive or doubtful sample to the reference laboratory for confirmation 


\section{References}

1. Batwala V, Magnussen P, Nuwaha F: Are rapid diagnostic tests more accurate in diagnosis of plasmodium falciparum malaria compared to microscopy at rural health centres? Malar $J$ 2010, 9: 349 .

2. World Health Organization: Guidelines for the treatment of malaria, second edition, 2010. [http://whqlibdoc.who.int/publications/2010/9789241547925 eng.pdf].

3. World Health Organization: World malaria report 2010. 2010. [http://whqlibdoc.who.int/publications/2010/9789241564106_eng.pdf].

4. European Commission: European standards in vitro diagnostic medical devices. 1998. [http://ec.europa.eu/enterprise/policies/european-standards/documents/harmonised-standardslegislation/list-references/iv-diagnostic-medical-devices/index_en.htm].

5. World Health Organization: Malaria Rapid Diagnostic Test Performance; Results of WHO product testing of malaria RDTs: Round $\mathbf{1}$ (2008). $\quad 2009$. [http://www.finddiagnostics.org/resource-centre/reports_brochures/malaria-diagnostics-report2009.html].

6. World Health Organization: Overview of the prequalification of diagnostics assessment process.

2010. [http://www.who.int/entity/diagnostics_laboratory/evaluations/pqdx_007_pq_overview_documen t_v3.pdf].

7. World Health Organization: Malaria Rapid Diagnostic Test Performance; Results of WHO product testing of malaria RDTs: Round 2 (2009). 2010. [http://www.finddiagnostics.org/resource-centre/reports brochures/malaria-diagnostic-testreport-round2.html].

8. Maltha J, Gillet P, Cnops L, Bottieau E, Van Esbroeck M, Bruggeman C, Jacobs J: Evaluation of the rapid diagnostic test SDFK40 (Pf-pLDH/pan-pLDH) for the diagnosis of malaria in a non-endemic setting. Malaria Journal 2011, 10: 7.

9. Moody AH, Chiodini PL: Non-microscopic method for malaria diagnosis using OptiMAL IT, a second-generation dipstick for malaria pLDH antigen detection. Br J Biomed Sci 2002, 59: 228-231.

10. Maltha J, Gillet P, Cnops L, Van Den Ende J, Van Esbroeck M, Jacobs J: Malaria rapid diagnostic tests: Plasmodium falciparum infections with high parasite densities may generate false positive Plasmodium vivax pLDH lines. Malar J 2010, 9: 198.

11. Hermans V, Monzote L, Van den Sande B, Mukadi P, Sopheak T, Gillet P, Jacobs J. Assessment of the comprehension of diagnostic and hazard symbols among health care workers in four international settings. Submitted.

12. Parekh BS, Anyanwu J, Patel H, Downer M, Kalou M, Gichimu C, Keipkerich BS, Clement N, Omondi M, Mayer O, Ou CY, Nkengasong JN: Dried tube specimens: a simple and costeffective method for preparation of HIV proficiency testing panels and quality control materials for use in resource-limited settings. J Virol Methods 2010, 163: 295-300.

13. Gillet P, Bosselaers K, Cnops L, Bottieau E, Van Esbroeck M., Jacobs J: Evaluation of the SD FK70 malaria Ag Plasmodium vivax rapid diagnostic test in a non-endemic setting. Malar $J$ 2009, 8: 129 .

14. Gillet P, van Dijk DP, Bottieau E, Cnops L, Van Esbroeck M, Jacobs J: Test characteristics of the SD FK80 Plasmodium falciparum/Plasmodium vivax malaria rapid diagnostic test in a non-endemic setting. Malar J 2009, 8: 262.

15. Van der Palen M, Gillet P, Bottieau E, Cnops L, Van Esbroeck M, Jacobs J: Test characteristics of two rapid antigen detection tests (SD FK50 and SD FK60) for the diagnosis of malaria in returned travellers. Malar $J$ 2009, 8: 90.

16. van Dijk DP, Gillet P, Vlieghe E, Cnops L, Van Esbroeck M, Jacobs J: Evaluation of the Palutop+4 malaria rapid diagnostic test in a non-endemic setting. Malar J 2009, 8: 293.

17. van Dijk DP, Gillet P, Vlieghe E, Cnops L, Van Esbroeck M, Jacobs J: Evaluation of the Immunoquick+4 malaria rapid diagnostic test in a non-endemic setting. Eur $J$ Clin Microbiol Infect Dis 2010, 29: 577-583.

18. Kaimbo K, Maertens K, Missotten L: Study of presbyopia in Zaire. Bull Soc Belge Ophtalmol 1987, 225 Pt 2: 149-156.

19. Patel VL, Branch T, Arocha JF: Errors in interpreting quantities as procedures: the case of pharmaceutical labels. Int J Med Inform 2002, 65: 193-211.

20. Rennie W, Phetsouvanh R, Lupisan S, Vanisaveth V, Hongvanthong B, Phompida S, Alday P, Fulache M, Lumagui R, Jorgensen P, Bell D, Harvey S: Minimising human error in malaria 
rapid diagnosis: clarity of written instructions and health worker performance. Trans $R$ Soc Trop Med Hyg 2007, 101: 9-18.

21. Gillet P, Mukadi P, Vernelen K, Van Esbroeck M, Muyembe JJ, Bruggeman C, Jacobs J: Comments to the results of the EQA session 2009/3 on malaria rapid diagnostic tests. 2011. [http://www.wivisp.be/ClinBiol/bckb33/activities/external_quality/rapports/_down/microbiologie/2010/10-3NCommentaar-malaria-sneltesten.pdf]. 
$-166-$ 


\title{
Hoofdstuk VII
}

\section{Samenvatting, algemene discussie en}

\author{
toekomst perspectieven
}




\section{Samenvatting, algemene discussie en toekomst perspectieven.}

Deze thesis heeft als doel enkele hiaten te dichten in de algemene kennis over test karakteristieken, de uitvoering door de gebruiker en de kwaliteit van de verpakking en de ingesloten gebruiksaanwijzing van malaria sneltesten (rapid diagnostic tests: RDTs).

In Hoofdstuk II werden de test karakteristieken geëvalueerd van 2 verschillende single-step RDTs gericht tegen $P$. vivax: SD FK70 malaria Ag Pv (enkel $P$. vivax detectie) en SD FK80 Pf/ Pv ( $P$. falciparum en $P$. vivax detectie). Hoewel de gevoeligheid van beide RDTs voor de detectie van $P$. vivax op gelijke voet staat met de gevoeligheid van de beste RDTs, zijn er toch enkele zaken die onze aandacht vragen. Enerzijds is deze gevoeligheid nog steeds niet hoog genoeg om de diagnosis van $P$. vivax op een betrouwbare manier uit te sluiten. Anderzijds stelden we vast dat - net als voor andere RDTs - de gevoeligheid ook sterk verminderd bij parasitemiën

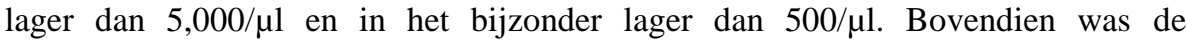
gevoeligheid voor de diagnose van $P$. falciparum (voor SD FK80) lager dan deze die werd verkregen met andere Pf - RDT, inclusief malaria RDTs die gebruik maken van identieke antilichamen geproduceerd door dezelfde fabrikant. Ten slotte vormt de kruisreactiviteit van $P$. falciparum met de $P$. vivax test lijn een belangrijke beperking voor de toepasbaarheid van deze RDTs, vooral de twee-band SD FK70. Weliswaar bleek het duidelijke en eenduidige onderscheid tussen $P$. vivax en $P$. ovale stalen zeer nuttig in een referentie setting. Momenteel wordt de SD FK70 gebruikt door het Central Laboratorium voor Klinische Biologie (CLKB, ITM) als back-up voor microscopische diagnose van beide species in afwachting van het resultaat van de species specifieke PCR.

Hoofdstuk III handelt over het voorkomen en de intensiteit van het prozone effect (vals negatief test resultaat bij hoge parasitemie) in een referentie setting en in een endemische setting. Er werd aangetoond dat HRP-2 RDTs in tegenstelling tot PfpLDH RDTs, getroffen kunnen worden door het prozone effect, weliswaar in verschillende mate en afhankelijk van lot tot lot. Prozone bleek zeldzaam te zijn in termen van intensiteit en frequentie: negatieve en "faint" test lijntjes waren goed voor een minderheid van de prozone-positieve stalen (18.2\% van 124 prozone positieve stalen) en het meest getroffen merk onder de geteste RDTs vertoonde prozone met negatieve of "faint" test lijntjes in 1.2\% van alle Plasmodium positieve stalen. Evenwel worden "faint" en zelfs "weak" test lijntjes vaak als negatief beschouwt door gebruikers zowel in endemische als non-endemische gebieden. Ook het inzetten van deze tests op het terrein - waar lokale labo's niet zijn uitgerust voor het verdunnen van stalen - is van belang [1-3]. Bovendien hangt de proportie van stalen met een verhoogde parasitemie ook af van lokale factoren zoals malaria 
endemiciteit en achtergrond immuniteit van de populatie. Deze huidige bevindingen moeten in het achterhoofd worden gehouden bij het maken van keuzes tussen het gebruik van ofwel Pf-pLDH ofwel HRP-2 gebaseerde RDTs.

Hoofdstuk IV beschrijft vals-positieve resultaten bij het uitvoeren van malaria RDTs met een andere buffer dan de buffer voorzien in de kit. Ongeveer driekwart van de malaria RDTs die werden geëvalueerd, vertoonden een vals-positief resultaat voor ten minste één staal en één gesubstitueerde buffer. Hoewel er geen gegevens bekend zijn over het gebruik van buffer vervanging, observeerden we zelf verschillende van deze buffer vervangingen op het terrein. Ook alumni collega's wezen ons op dit probleem. Het resultaat van deze studie zou kunnen bijdragen tot verdere aanpassingen in het ontwerp van malaria RDT kits, zoals het toevoegen van meer dan één buffer flacon aan de malaria RDT kits en het vermelden van een waarschuwing in de gebruiksaanwijzing omtrent het belang van het gebruik van de voorziene buffer.

In Hoofdstuk $\mathbf{V}$ worden de resultaten gerapporteerd van een externe kwaliteitscontrole rond het gebruik van malaria RDTs in een non-endemische setting. Deze tonen een uitstekende analytische prestatie aan van de malaria RDTs, maar wijzen ook op kleine doch consistente fouten in de interpretatie van de test resultaten, deels veroorzaakt door foute omschrijvingen in de gebruiksaanwijzing. De antwoorden op de vragenlijst bevestigden de lage blootstelling van het labo personeel aan Plasmodium positieve stalen, maar als belangrijkste punt bracht dit een vertrouwen aan het licht in de RDTs als eerste en enige diagnostisch middel voor de diagnose van malaria buiten de openingsuren van het labo. Gezien de mogelijke diagnostische fouten en de technische beperkingen van malaria RDTs, moet deze praktijk absoluut vermeden worden.

In Hoofdstuk VI werden malaria RDTs geëvalueerd voor hun adequate en correcte verpakking, ontwerp en etikettering van dozen en componenten alsook voor de leesbaarheid en accuraatheid van de aangeboden informatie. Talrijke tekortkomingen werden opgemerkt, zelfs voor CE-gelabelde, ISO 13485 gecertificeerde producten en producten opgenomen in de Wereldgezondheidsorganisatie (WGO)/FIND aankooplijst. De meest relevante tekortkomingen waren de dubbelzinnige labels van de resultaten schaal en het onvoldoende labellen van de buffer flacons. De gebruiksaanwijzing was moeilijk leesbaar en de typografie was niet gebruiksvriendelijk. Essentiële onderwerpen als bio veiligheidsmaatregelen waren heel vaak niet vermeld en er werd onvolledige en incorrecte informatie vastgesteld bij de afbeeldingen en de interpretatie van de test lijn resultaten. De meeste van deze fouten kunnen echter heel gemakkelijk worden opgelost en duidelijke instructies van professionals en regelgevende instanties kunnen de kwaliteit van RDT verpakkingen en gebruiksaanwijzingen verbeteren 
tegen een lage additionele kostprijs. Leidinggevende instanties zoals de (WGO) of de Europese Gemeenschap zouden deze verbeteringen kunnen aanmoedigen en versterken, bijvoorbeeld als deel van de pre kwalificatie eisen of door het toevoegen aan de zogenoemde "Annex II producten" voor dewelke de EU richtlijn over in vitro diagnostica (IVD) lot testing voorschrijft vooraleer het product op de markt wordt gebracht [4-7].

Deze huidige studies tonen duidelijk aan dat malaria RDTs een essentieel onderdeel van malaria diagnose vertegenwoordigen, zowel in endemische als non-endemische gebieden. De relevante conclusies uit bovenstaande hoofdstukken bepalen de analytische, pre- en post-analytische karakteristieken van malaria RDTs. Als onderdeel van de analytische karakteristieken toonden we de diagnostische karakteristieken aan van twee single-step RDTs voor de detectie van $P$. vivax en bestudeerden we technische factoren gelinkt aan het ontwerp van RDTs zoals het prozone effect en vals-positieve resultaten na buffer vervanging. Beide factoren zijn ook deels gerelateerd aan het ontwerp van de immuno-chromatografische strips, waarbij geen wasstap wordt uitgevoerd om blokkerende antigenen of aspecifieke interacties te verwijderen. Pre-analytische en post-analytische karakteristieken werden duidelijk door de externe kwaliteitscontrole van malaria RDTs. Deze bracht fouten aan het licht met betrekking tot (i) indicaties en diagnostische strategie (preanalyse) en (ii) interpretatie van de resultaten en rapportering (post-analyse). Aangezien fouten met betrekking tot de interpretatie en de rapportering van de resultaten verband hielden met de foutieve instructies van de producent, verkenden we de RDT verpakking en gebruiksaanwijzing verder. Dit onderzoek wees op een aantal tekortkomingen die eenvoudig en tegen een aanvaardbare prijs verholpen kunnen worden door enkele simpele maatregelen en procedures te nemen. Deze kunnen op hun beurt worden aangemoedigd door werkdocumenten uitgegeven door professionele organisaties.

In de huidige studie identificeerden we ook enkele bijkomende onderzoeksvragen. Hoofdstuk II en III gaan over de studie van testkarakteristieken van Pf-pLDH gebaseerde RDTs. De SD FK40 (Standard Diagnostics Inc., Hagal-Dong, Korea) welke een Pf-pLDH/pan-pLDH gebaseerde RDT is, werd reeds geëvalueerd in een labo setting [8]. Evaluaties van CareStart Pf-pLDH/pan-pLDH (Access Bio Inc, Monmouth Junction, NJ, USA) en SD FK90 (Standard Diagnostics Inc., HagalDong, Korea), een gecombineerde HRP-2 en Pf-pLDH malaria RDT, zijn lopende. Deze laatste RDT zou - mits excellente test karakteristieken voor een redelijke prijs - een goede kandidaat kunnen zijn voor het terrein: het is geweten dat HRP-2 gedurende enkele weken na de behandeling van een $P$. falciparum infectie kan persisteren in het bloed [9]. De gecombineerde detectie van HRP-2 en Pf-pLDH door een RDT zoals SD FK90 zou niet enkel het prozone effect kunnen omzeilen, maar geeft ook de mogelijkheid een onderscheid te maken tussen een huidige en een 
recente of behandelde $P$. falciparum infectie. Daarenboven, in Hoofdstuk II observeerden we occasionele kruisreacties van $P$. falciparum met $P$. vivax-specifieke $\mathrm{Pv}$-pLDH, reden waarom we besloten dit fenomeen verder te onderzoeken: we onderwierpen een panel van $P$. vivax $\mathrm{RDTs}$ aan een set van $P$. falciparum stalen met hoge parasitemie. Vals positieve $\mathrm{Pv}$-pLDH lijntjes werden geobserveerd in 6/9 RDTs met frequenties gaande van $8.2 \%$ tot $29.1 \%$ voor 85 stalen [10].

Het probleem van de leesbaarheid van de gebruiksaanwijzing van RDTs beschreven in Hoofdstuk VI deed vragen rijzen over de interpretatie en de kennis van diagnostische symbolen ontwikkeld door ISO 15223-1:2007 en EN 980:2008, die werden geïntroduceerd om het probleem van talenkennis van de mogelijke gebruikers te omzeilen. Samen met onze partners in het Zuiden voerden we een enquête uit bij professionele gezondheidswerkers in de Democratische Republiek of the Congo, Cuba, Cambodja en België met het oog op de evaluatie van hun actuele en contextuele kennis van diagnostische (gevaren) symbolen. Voor alle deelnemers samen was de bewezen kennis van de symbolen zeer klein. Enkel drie van de 20 symbolen werden voor $67 \%$ correct gescoord (ISO 3864 criteria). Hoewel de twee gevaren symbolen wel herkend werden als gevaar bij de meeste deelnemers, haalde deze toch een te lage score $(<50 \%)$. De slechte kennis van de symbolen bewijst zeer duidelijk de nood aan specifieke trainingen omtrent het correct gebruik van RDTs en in-vitro diagnostica in het algemeen. Fabrikanten zouden in de gebruiksaanwijzing een verklarende lijst moeten insluiten om de betekenis van de symbolen te verduidelijken [11].

Toekomstig onderzoek zal gericht worden op het gebruik van RDTs in malaria endemische ontwikkelingslanden. In zo'n omgeving vragen externe kwaliteitscontroles zoals deze beschreven in Hoofdstuk $\mathrm{V}$ alternatieve test specimens die bestand zijn tegen de tropische temperaturen en vochtigheid. Recent werden "dried tube specimens" (DTS) ontwikkeld als een simpel en stabiel transportmiddel voor sera voor de detectie van HIV-antilichamen [12]. Een gelijkaardig systeem zal worden ontwikkeld voor bloedstalen geïnfecteerd met Plasmodium species; Dit systeem zal worden gevalideerd samen met onze partners in Lima, Peru.

Waarnemingen uit Hoofdstuk II en ook in andere RDT evaluaties uitgevoerd door CLKB geven aan dat bijna één op drie positieve test lijnen een zwakke tot zeer zwakke intensiteit vertonen ("weak" en "faint") [8,13-17]. Het probleem van de interpretatie van deze tests als negatief zou dus groter kunnen zijn dan oorspronkelijk verwacht. In Afrikaanse landen wordt presbyopia (of verziendheid) vaak niet herkend. Bij deze bevolking zou de prevalentie zelfs hoger zijn dan in de Europese en zou deze aandoening op jongere leeftijd [18,19] voorkomen. Een mogelijke rol van verziendheid als een oorzaak van het foutief aflezen van RDT resultaten werd al vooropgesteld [20], maar de mate waarin is tot nu toe nog niet 
gekend. Daarom zetten wij momenteel een studie op met onze partners in Kinshasa om de prevalentie van presbyopia bij RDT gebruikers na te gaan.

Waarnemingen gedaan in Hoofdstuk VI over de tekortkomingen in verpakkingen, labels en gebruiksaanwijzingen van malaria RDTs vragen een verdere opvolging en moeten worden uitgebreid naar andere IVDs. Dit werk zal worden gerealiseerd door het QUAMED netwerk. Dit netwerk - georganiseerd door ITM - brengt operationele en academische organisaties van Noord en Zuid samen met als objectief bij te dragen aan een universele toegang tot kwaliteitsvolle medicatie en diagnostica.

De resultaten van de studies uitgevoerd voor deze thesis als ook andere publicaties gerelateerd aan het gebruik van RDTs op het terrein zijn samengebracht in twee samenvattende tabellen. Ze werden gecompileerd en gepubliceerd als onderdeel van het didactisch verslag geadresseerd aan de deelnemers van de externe kwaliteitscontrole beschreven in Hoofdstuk V [21]. Tabel 1 bevat de additionele waarde van het gebruik van malaria RDTs in de diagnose van malaria in een nonendemische setting, met zijn sterktes, zwaktes en bijzonderheden van de verschillende antigenen en Plasmodia. Tabel 2 bevat de "Do's" en de "Don'ts" die moeten worden in acht gehouden bij het dagelijks gebruik van malaria RDTs in het labo.

We kunnen besluiten dat malaria RDTs zeker een voordeel bieden wat betreft malaria diagnose. Een gezonde kennis van zijn karakteristieken en beperkingen door de gebruiker zal ook de diagnostische waarde van de RDTs doen toenemen. 
Tabel 1. Wat dragen malaria RDTs bij tot de diagnose in een non-endemische setting?

Vereisten malaria diagnosis

Tijdige bevestiging of uitsluiting van malaria diagnose met een dadelijke verwijzing in geval van enige twijfel

Onderscheid $P$. falciparum (mogelijks levensbedreigend) en non-falciparum species

Bepalen van parasitaire densiteit, in het bijzonder herkennen van kritische waarde ( $>2 \%$ rode bloedcellen geïnfecteerd)

Herkennen van P. falciparum stadia en pigment

\section{Bijdrage van MRDTs Opmerkingen}

Uitstekende gevoeligheid, zeker voor $P$. falciparum $>100$ parasieten $/ \mu 1$

Aanzienlijke bijdrage bij malaria diagnose

Vals negatieve resultaten voor $P$. falciparum bij lage parasitaire densiteit $(<100 / \mu 1)$, af en toe ook hoger

Sommige HRP-2 mutaties worden niet opgepikt.

Prozone effect is zeer zeldzaam,

Sluiten malaria niet uit , maar het gebeurt in malaria RDTs microscopie nog steeds noodzakelijk gebaseerd op HRP-2

Matige bijdrage voor diagnose van $P$. vivax, slechts kleine bijdrage voor diagnose van $P$. ovale en P. malariae het identificeren van $P$. falciparum
Aanzienlijke bijdrage bij

Menginfecties zijn zeldzaam maar kunnen niet worden uitgesloten als P. falciparum- en Pan-species antigen lijntjes zichtbaar zijn

Intensiteit van de testlijntjes zijn een aanwijzing voor parasitaire densiteit maar veel overlapping

Geen

Een enkele HRP-2 lijn kan wijzen op infectie met lage parasitemie

Geen 
Tabel 2a DO's en DON'Ts bij het gebruik van malaria RDTs (endemische en non-endemische setting).

\section{DO}

Controleer de controlelijn - wanneer niet zichtbaar, herhaal de test.

Herhaal een negatieve RDT in geval van hoog vermoeden van malaria.

Beschouw elke lijn, hoe zwak ook, als een positief resultaat.

Respecteer het bloed- en buffervolume

\section{Opmerkingen}

Afwezigheid van de controlelijn wijst op een invalide test, resultaat kan niet worden geïnterpreteerd.

Herhaal elke 8-12 uur voor 4 opeenvolgende keren gedurende 36 uur om malaria uit te sluiten.

Elke zichtbare lijn is een positieve lijn.

Te weinig bloed geeft kans op een vals-negatief resultaat.

Te veel bloed geeft meer achtergrond, moeilijker te interpreteren.

Te veel bloed verhoogt de kans op prozone-effect.

\section{Opmerkingen}

Te lage temperaturen beschadigen het colloidale goud.

Te vroeg aflezen, verhoogt kans op vals-negatieve resultaten.

Te laat verhoogt kans op vals-positieve resultaten.

RDTs gebaseerd op HRP-2 (P. falciparum) kunnen tot enkele weken na de behandeling positief blijven.

Gametocyten brengen pLDH en aldolase to expressie.

Tabel 2b DO's en DON'Ts bij het gebruik van malaria RDTs (addendum voor non-endemische setting).

\section{DO}

Combineer een RDT ALTIJD met microscopie!

Gebruik een transfer-pipet.

Bij twijfel en bij een positief staal: zend naar referentie labo voor bevestiging.

\section{Opmerkingen}

De collectoren en pipetjes voorzien bij de RDTs zijn klein en moeilijk te hanteren.

Voor België: Aanvraagformulieren en instructies: https://www.iph.fgov.be/epidemio/epinl/plabnl/N_Plasmodium.pdf https://www.iph.fgov.be/epidemio/epifr/plabfr/F_Plasmodium.pdf 
References

1. Batwala V, Magnussen P, Nuwaha F: Are rapid diagnostic tests more accurate in diagnosis of plasmodium falciparum malaria compared to microscopy at rural health centres? Malar $J$ 2010, 9: 349 .

2. World Health Organization: Guidelines for the treatment of malaria, second edition, 2010. [http://whqlibdoc.who.int/publications/2010/9789241547925 eng.pdf].

3. World Health Organization: World malaria report 2010. 2010. [http://whqlibdoc.who.int/publications/2010/9789241564106 eng.pdf].

4. European Commission: European standards in vitro diagnostic medical devices. 1998. [http://ec.europa.eu/enterprise/policies/european-standards/documents/harmonised-standardslegislation/list-references/iv-diagnostic-medical-devices/index_en.htm].

5. World Health Organization: Malaria Rapid Diagnostic Test Performance; Results of WHO product testing of malaria RDTs: Round 1 (2008). [http://www.finddiagnostics.org/resource-centre/reports_brochures/malaria-diagnostics-report2009.html].

6. World Health Organization: Overview of the prequalification of diagnostics assessment process.

2010. [http://www.who.int/entity/diagnostics_laboratory/evaluations/pqdx_007_pq_overview_documen t v3.pdf].

7. World Health Organization: Malaria Rapid Diagnostic Test Performance; Results of WHO product testing of malaria RDTs: Round 2 (2009). 2010. [http://www.finddiagnostics.org/resource-centre/reports brochures/malaria-diagnostic-testreport-round2.html].

8. Maltha J, Gillet P, Cnops L, Bottieau E, Van Esbroeck M, Bruggeman C, Jacobs J: Evaluation of the rapid diagnostic test SDFK40 (Pf-pLDH/pan-pLDH) for the diagnosis of malaria in a non-endemic setting. Malaria Journal 2011, 10: 7.

9. Moody AH, Chiodini PL: Non-microscopic method for malaria diagnosis using OptiMAL IT, a second-generation dipstick for malaria pLDH antigen detection. Br J Biomed Sci 2002, 59: 228-231.

10. Maltha J, Gillet P, Cnops L, Van Den Ende J, Van Esbroeck M, Jacobs J: Malaria rapid diagnostic tests: Plasmodium falciparum infections with high parasite densities may generate false positive Plasmodium vivax pLDH lines. Malar J 2010, 9: 198.

11. Hermans V, Monzote L, Van den Sande B, Mukadi P, Sopheak T, Jacobs J. Assessment of the comprehension of diagnostic and hazard symbols among health care workers in four international settings. Submitted.

12. Parekh BS, Anyanwu J, Patel H, Downer M, Kalou M, Gichimu C, Keipkerich BS, Clement N, Omondi M, Mayer O, Ou CY, Nkengasong JN: Dried tube specimens: a simple and costeffective method for preparation of HIV proficiency testing panels and quality control materials for use in resource-limited settings. $J$ Virol Methods 2010, 163: 295-300.

13. Gillet P, Bosselaers K, Cnops L, Bottieau E, Van Esbroeck M., Jacobs J: Evaluation of the SD FK70 malaria Ag Plasmodium vivax rapid diagnostic test in a non-endemic setting. Malar $J$ 2009, 8: 129.

14. Gillet P, van Dijk DP, Bottieau E, Cnops L, Van Esbroeck M, Jacobs J: Test characteristics of the SD FK80 Plasmodium falciparum/Plasmodium vivax malaria rapid diagnostic test in a non-endemic setting. Malar J 2009, 8: 262.

15. Van der Palen M, Gillet P, Bottieau E, Cnops L, Van Esbroeck M, Jacobs J: Test characteristics of two rapid antigen detection tests (SD FK50 and SD FK60) for the diagnosis of malaria in returned travellers. Malar J 2009, 8: 90.

16. van Dijk DP, Gillet P, Vlieghe E, Cnops L, Van Esbroeck M, Jacobs J: Evaluation of the Palutop+4 malaria rapid diagnostic test in a non-endemic setting. Malar J 2009, 8: 293.

17. van Dijk DP, Gillet P, Vlieghe E, Cnops L, Van Esbroeck M, Jacobs J: Evaluation of the Immunoquick+4 malaria rapid diagnostic test in a non-endemic setting. Eur $J$ Clin Microbiol Infect Dis 2010, 29: 577-583.

18. Kaimbo K, Maertens K, Missotten L: Study of presbyopia in Zaire. Bull Soc Belge Ophtalmol 1987, 225 Pt 2: 149-156.

19. Patel VL, Branch T, Arocha JF: Errors in interpreting quantities as procedures: the case of pharmaceutical labels. Int J Med Inform 2002, 65: 193-211.

20. Rennie W, Phetsouvanh R, Lupisan S, Vanisaveth V, Hongvanthong B, Phompida S, Alday P, Fulache M, Lumagui R, Jorgensen P, Bell D, Harvey S: Minimising human error in malaria 
rapid diagnosis: clarity of written instructions and health worker performance. Trans $R$ Soc Trop Med Hyg 2007, 101: 9-18.

21. Gillet P, Mukadi P, Vernelen K, Van Esbroeck M, Muyembe JJ, Bruggeman C, Jacobs J: Comments to the results of the EQA session 2009/3 on malaria rapid diagnostic tests. 2011. [http://www.wivisp.be/ClinBiol/bckb33/activities/external_quality/rapports/_down/microbiologie/2010/10-3NCommentaar-malaria-sneltesten.pdf]. 


\section{List of publications}

\section{Articles submitted or in press:}

1. Mukadi P, Gillet P, Lukuka A, Atua B, Kahodi S, Lokombe J, Muyembe JJ, Jacobs J: External quality assessment of malaria microscopy in the Democratic Republic of the Congo. Submitted.

2. Gillet P, Scheirlinck A, Stokx J, De Weggheleire A, Chaúque H, de Jesus Varela Canhanga O, Tadeu B, das Dores Mosse C, Tiago A, Mabunda S, Bruggeman C, Bottieau E, Jacobs J: Prozone in malaria rapid diagnostic test: how many cases are missed? Submitted.

3. Hermans V, Monzote L, Van den Sande B, Mukadi P, Sopheak T, Gillet P, Jacobs J: Assessment of the knowledge of graphical symbols labeled on malaria rapid diagnostic tests in four international settings. Submitted.

4. Stokx J, Gillet P, De Weggheleire A, Casas E, Maendaenda R, Beulane A, Jani I, Kidane S, das Dores Mosse C, Jacobs J, Bottieau E: Seroprevalence of transfusion-transmissible infections and evaluation of the pre-donation screening performance at the Provincial Hospital of Tete, Mozambique. BMC Infectious Diseases, in press.

\section{Articles published:}

1. Cnops L, Boderie M, Gillet P, Van Esbroeck M, Jacobs J: Rapid diagnostic tests as a source of DNA for Plasmodium species-specific real-time PCR. Malar J 2011,10:67

2. Gillet P, Maltha J, Hermans V, Ravinetto R, Bruggeman C, Jacobs J: Malaria rapid diagnostic kits: quality of packaging, design and labelling of boxes and components and readability and accuracy of information inserts. Malar J 2011, 10:39.

3. Maltha J, Gillet P, Cnops L, Bottieau E, Van Esbroeck M, Bruggeman C, Jacobs J: Evaluation of the rapid diagnostic test SDFK40 (Pf-pLDH/pan-pLDH) for the diagnosis of malaria in a non-endemic setting. Malaria Journal 2011, 10: 7.

4. Gillet P, Mukadi P, Vernelen K, Van Esbroeck M, Muyembe J-J, Bruggeman C, Jacobs J: External quality assessment on the use of malaria rapid diagnostic tests in a non-endemic setting. Malar J 2010, 9: 359 .

5. Gillet P, Mukadi P, Vernelen K, Van Esbroeck M, Muyembe J-J, Bruggeman C, Jacobs J: Comments to the results of the EQA session 2009/3 on malaria rapid diagnostic tests. 2010. [http://www.wivisp.be/ClinBiol/bckb33/activities/external_quality/rapports/_down/microbiologie/2010/10-3NCommentaar-malaria-sneltesten.pdf]

6. Gillet P, Mori M, Van Den Ende J, Jacobs J: Buffer substitution in malaria rapid diagnostic tests causes false-positive results. Malar J 2010, 9: 215 .

7. Maltha J, Gillet P, Cnops L, Van Den Ende J, Van Esbroeck M, Jacobs J: Malaria rapid diagnostic tests: Plasmodium falciparum infections with high parasite densities may generate false positive Plasmodium vivax pLDH lines. Malar J 2010, 9: 198.

8. Maltha J, Gillet P, Bottieau E, Cnops L, Van Esbroeck M, Jacobs J: Evaluation of a rapid diagnostic test (CareStart Malaria HRP-2/pLDH (Pf/pan) Combo Test) for the diagnosis of malaria in a reference setting. Malar J 2010, 9: 171. 
9. van Dijk DP, Gillet P, Vlieghe E, Cnops L, Van Esbroeck M, Jacobs J: Evaluation of the Immunoquick+4 malaria rapid diagnostic test in a non-endemic setting. Eur $J$ Clin Microbiol Infect Dis 2010, 29: 577-583.

10. van Dijk DP, Gillet P, Vlieghe E, Cnops L, Van Esbroeck M, Jacobs J: Evaluation of the Palutop+4 malaria rapid diagnostic test in a non-endemic setting. Malar J 2009, 8: 293.

11. Gillet P, Mori M, Van Esbroeck M, Van Den Ende J, Jacobs J: Assessment of the prozone effect in malaria rapid diagnostic tests. Malar J 2009, 8: 271.

12. Gillet P, van Dijk DP, Bottieau E, Cnops L, Van Esbroeck M, Jacobs J: Test characteristics of the SD FK80 Plasmodium falciparum/Plasmodium vivax malaria rapid diagnostic test in a non-endemic setting. Malar J 2009, 8: 262.

13. Gillet P, Bosselaers K, Cnops L, Bottieau E, Van Esbroeck M., Jacobs J: Evaluation of the SD FK70 malaria Ag Plasmodium vivax rapid diagnostic test in a non-endemic setting. Malar $J$ 2009, 8: 129.

14. Van der Palen M, Gillet P, Bottieau E, Cnops L, Van Esbroeck M, Jacobs J: Test characteristics of two rapid antigen detection tests (SD FK50 and SD FK60) for the diagnosis of malaria in returned travellers. Malar J 2009, 8: 90.

15. Guthmann JP, Ampuero J, Fortes F, van Overmeir C, Gaboulaud V, Tobback S, Dunand J, Saraiva N, Gillet P, Franco J, Denoncin A, van Herp M, Balkan S, Dujardin JC, D'Alessandro U, Legros D: Antimalarial efficacy of chloroquine, amodiaquine, sulfadoxine-pyrimethamine, and the combinations of amodiaquine + artesunate and sulfadoxine-pyrimethamine + artesunate in Huambo and Bie provinces, central Angola. Trans R Soc Trop Med Hyg 2005, 99: $485-492$.

16. Haubruge E, Chasseur C, Debouck C, Begaux F, Suetens C, Mathieu F, Michel V, Gaspar C, Rooze M, Hinsenkamp M, Gillet P, Nolard N, Lognay G: The prevalence of mycotoxins in Kashin-Beck disease. Int Orthop 2001, 25: 159-161.

17. Gillet P, Kessler W, de Meyere S, Garcia I, Maossede J: Resistance in vivo of Plasmodium falciparum to chloroquine in the south-west of Chad. Medical News 1992, 1: 13-17.

\section{Books or chapters:}

1. Suetens C, Mathieu F, Begaux F, Gillet P, Kanyandekwe P, Goyens P, Tserin D, He L, Lagrange M, Durand M-C, Chasseur C: Second epidemiological study: prevention trial (chapter 4.6). Big Bone Disease: A multidisciplinary approach of Kasing-Beck disease in Tibet Autonomous Region (P.R. China) (pp. 67-74). Gembloux, Belgique: Les Presses Agronomiques de Gembloux.

2. Suetens C, Chasseur C, Claus W, Bally P, Rapten S, Wangla R, Rinchen L, Wangdu L, Drolkar P; He L, Tserin D, Tserin N, Gillet P, Eddyani M, Kanyandekwe P, Goyens P, Mathieu F: Third epidemiological study: Clinical trial based on nutritional issues (chapter 4.8). Big Bone Disease: A multidisciplinary approach of Kasing-Beck disease in Tibet Autonomous Region (P.R. China) (pp. 81-82). Gembloux, Belgique: Les Presses Agronomiques de Gembloux.

3. Chasseur C, Lognay G, Suetens C, Rapten S, Gillet P, Kanyandekwe P, He L. Drolkar P, Wangla R, Begaux F, Haubruge E, Rinchen L, Wangdu L, Claus W, Mathieu F: The fungal hypotheses (chapter 5.1). Big Bone Disease: A multidisciplinary approach of Kasing-Beck disease in Tibet Autonomous Region (P.R. China) (pp. 85-99). Gembloux, Belgique: Les Presses Agronomiques de Gembloux. 


\section{Curriculum Vitae}

Philippe Gillet was born in Sint-Joost-Ten-Node, Belgium on July 25, 1965. He graduated from secondary school at the Institute Sainte-Marie in Brussels in 1983. In 1988 he obtained his Bachelor degree in Clinical Biology with distinction at the Paul Lambin Institute in Brussels. The same year, he started a Master of Sciences in Biomedical Technology at the Catholic University of Leuven. In 1990, he obtained his Master degree with great distinction and started the certificated Postgraduate Course in Tropical Clinical Biology at the Institute of Tropical Medicine in Antwerp. He obtained his certificate in 1991 with distinction. From 1991 to 1995 he worked for Doctors Without Borders - Belgium in Chad as provincial laboratory manager and in Cambodia as tutor at the Central School for Management, section of laboratory technicians. In 1995 he started a Master in Public Health Methodology at the Public Health School of the Free University of Brussels. After obtaining this Master degree with great distinction, he worked subsequently for the Belgium Red Cross in Rwanda, the European Agency for Development and Health in the republic of Zaire and Doctors Without Borders in Angola and Tibet Autonomous Region until 2000. In 2000, he started for one year as a practical assistant at the Institute of Tropical Medicine, Antwerp. In 2001, he went back to the field with Doctors Without Borders to work in Ethiopia and Angola. He returned to the Institute as practical assistant in 2003. In December 2009, he was selected for a SOFI-A mandate of the ITM to complete his $\mathrm{PhD}$. 


\section{Acknowledgments}

I would like to thank all people who participated directly or indirectly in this thesis:

In the first place I would like to thank my co-supervisor, Prof. dr. Jan Jacobs, who gave me the opportunity to start this research and who showed a lot of patience and wisdom in advising me, reading and editing all projects, papers and manuscripts over and over again, despite a huge workload. His guidance helped me at all times, during research and the writing of this thesis. I can not imagine having a better advisor and mentor for this work. I also gratefully acknowledge Prof. dr. Cathrien Bruggeman, my supervisor in Maastricht for her help and enthusiasm, despite our geographical separation. Special thanks as well to all colleagues at the Institute of Tropical Medicine in Antwerp: dr. Marjan Van Esbroeck, director of the Central Laboratory for Clinical Biology and all laboratory staff. I am very grateful to Lieselotte Cnops for conducting the PCR's and coaching me in the molecular aspects of malaria diagnosis, as well as to Agnes D'Hondt, Denise Boons, Pieter Guetens and Anne-Mie Feyens for sample collection and malaria microscopy. I am addressing many thanks as well to my colleagues and students at the Unit of Tropical Laboratory Medicine: Mirna Van der Palen, Katrien Bosselaers, David van Dijk and Jessica Maltha who share the same interest in malaria rapid diagnostic tests. I am also indebted to Hilde De Boeck and Birgit De Smet for encouraging me and creating a pleasant working atmosphere and to Annelies Scheirlinck for her substitution [not literally but figuratively as a (malaria) "buffer"] in teaching and research activities. I would like to thank my colleagues of the practical teaching Luc Boel and Idzi Potters for their patience and their availability to take over some practical sessions. A special thanks goes to the project team in Tete, Mozambique: to Dr. Emmanuel Bottieau who introduced me in Mozambique and supervised the clinical field work in Tete together with dr. Anja De Weggheleire; and to Annelies Scheirlinck and Jocelijn Stokx who assured reliable and accurate study results. In addition, I am grateful to the medical, paramedical and laboratory staff of the Provincial Hospital of Tete for their clinical and laboratory assistance. Likewise, I want to thank the colleagues of the INRB in Kinshasa for their help in the present $\mathrm{PhD}$ and their involvement in other ongoing collaborations in malaria research: in particular, I want to thank Pierre Mukadi and Prof. dr. JJ Muyembe for their support and empathy. Accross the ocean, my thanks go to dr. Dioni Gamboa for fruitful discussion, concrete advices and charismatic interest in my work. I also gratefully acknowledge the ITM's Secondary Funding Sofi-A program that made this thesis possible and the interests and stimuli from the part of the Department of Clinical Sciences, in particular by Prof. dr. Van den Ende. Further, I would also like to thank my wife, Catherine and our children Benjamin and Emmanuel for their support, comprehension and patience. Finally, I would like to thank the malaria patients for their cooperation, with the hope that this work will contributed to improve the malaria diagnosis and care. 